\title{
Patterned Alignment of Liquid Crystals by Microrubbing
}

\begin{abstract}
A new route towards wide viewing angle flat panel displays
\end{abstract}

Soney Varghese 


\title{
CIP-DATA LIBRARY TECHNISCHE UNIVERSITEIT EINDHOVEN
}

\author{
Varghese, Soney
}

Patterned Alignment of Liquid Crystal by Microrubbing-A new route towards wide viewing angle flat panel displays. / by Soney Varghese.-Eindhoven: Technische Universiteit Eindhoven, 2005.

Proefschrift.-ISBN 90-386-2776-9

NUR 913

Subject headings: liquid crystal displays; LCD / microrubbing / molecular orientation; vertical alignment / electro-optical effect; viewing angle / polyimides / liquid crystals; phase separation

Trefwoorden: vloeibare kristalschermen; LCD / microwrijven / moleculaire orientatie; verticale uitlijning / elektro-optisch effect; kijkhoek / polyimiden / vloeibare kristallen; fasescheiding

An electronic version of this thesis is available on the website of the Eindhoven University of Technology in PDF-format (http://www.tue.nl/bib).

Copyright (C) 2005, Soney Varghese

Cover design by Chris van Heesch and Soney Varghese

Printed by University Press Facilities, Eindhoven, The Netherlands

Cover: The picture on the front cover shows the intensity profile of the sub-pixel fabricated by the $\mu$-rubbing process in the vertically aligned mode. The images on the spine of the thesis shows a liquid crystal cell viewed between crossed polarizers of an optical microscope that was created through a $\mu$-rubbing process. The switching of the liquid crystal cell is shown as a function of voltage. 


\title{
Patterned Alignment of Liquid Crystals by Microrubbing
}

\author{
A new route towards wide viewing angle \\ flat panel displays
}

PROEFSCHRIFT

\author{
ter verkrijging van de graad van doctor aan de \\ Technische Universiteit Eindhoven, op gezag van de \\ Rector Magnificus, prof.dr.ir. C.J. van Duijn, voor een \\ commissie aangewezen door het College voor \\ Promoties in het openbaar te verdedigen \\ op woensdag 1 juni 2005 om 16.00 uur
}

door

Soney Varghese

geboren te Ezhupunna, India 
Dit proefschrift is goedgekeurd door de promotoren:

prof.dr. D.J. Broer

en

prof.dr. P.J. Lemstra

Copromotor:

dr.ir. C.W.M. Bastiaansen

The work described in this dissertation was carried out at Polymers in Information and Communication Technology (PICT) group at Polymer Technology within the Faculty of Chemistry and Chemical Engineering of the Eindhoven University of Technology. The research has been supported by the "Netherlands organization for international cooperation in higher education (NUFFIC)" within the framework of the "Joint financing program for cooperation in higher education (MHO). 
Dedicated to my beloved parents

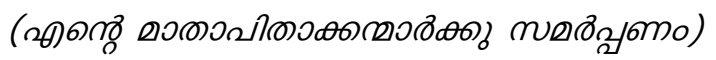





\section{Contents}

\section{Chapter 1 General Introduction}

1.1 Information display 1

1.2 Liquid crystal display 2

1.2.1 Twisted nematic display 3

1.2.2 Super twisted nematic display 5

$\begin{array}{ll}\text { 1.2.3 Vertically aligned display } & 6\end{array}$

1.2.4 In-plane switching mode $\quad 7$

1.3 Viewing angle of LCDs 9

$\begin{array}{ll}\text { 1.3.1 Optical compensation film } & 10\end{array}$

1.3.2 Multidomain liquid crystal display 11

$\begin{array}{lll}1.4 & \text { Objectives } & 14\end{array}$

1.5 Scope of the thesis 14

1.6 References 16

Chapter 2 Patterned Alignments of Liquid Crystals by $\mu$-Rubbing

2.1 Introduction 19

2.2 Optical simulations:-Theory 20

2.3 Experimental 21

2.3.1 Materials 21

2.3.2 $\mu$-Rubbing setup 21

2.3.3 Cell construction $\quad 22$

2.3.4 Characterization 23

2.3.5 Electro-optical characterization 23

$\begin{array}{ll}\text { 2.3.6 Pretilt angle measurement } 23 & 23\end{array}$

2.4 Results and discussion $\quad 24$

2.4.1 $\mu$-Rubbing of unrubbed and pre-rubbed polyimide: 24

Surface characterization 
2.4.2 Alignment of LCs on $\mu$-rubbed surface 28

2.4.3 Pretilt angle measurements: Conoscopic technique 32

2.4.4 Alignment of LCs in an applied field 33

2.4.5 Optical simulations 37

2.4.5.1 Director orientations and transmittance 37

2.4.6 Electro-optical characterization 43

2.4.7 Electro-optical characteristics: - Simulations 46

2.4.8 Multidomain configuration: - Planar polyimide 47

2.5 Conclusions 50

2.6 References 51

\section{Chapter 3 Microrubbing of Homeotropic Polyimide}

3.1 Introduction $\quad 53$

3.2 Experimental 54

3.2.1 Materials and sample preparation $\quad 54$

3.2.2 Characterization 55

3.2.3 Line scanning $\quad 55$

3.2.4 Pretilt angle measurement 56

3.3 Results and discussion $\quad 56$

3.3.1 Morphology of patterns 56

3.3.2 Multi-configurations $\quad 57$

3.3.3 Pretilt angle measurements $\quad 59$

3.3.4 Optical simulations: - Director orientations and transmittance 61

3.3.5 Electro-optical characteristics $\quad 67$

3.3.6 Electro-optical characteristics: - Simulations 69

$\begin{array}{ll}\text { 3.3.7 Alignment mechanism } & 70\end{array}$

$\begin{array}{lll}3.4 & \text { Conclusions } & 72\end{array}$

$\begin{array}{lll}3.5 & \text { References } & 72\end{array}$ 
Chapter 4 Multidomain Twisted Nematic Liquid Crystal Display by $\mu$-Rubbing

4.1 Introduction

4.2 Experimental

4.2.1 Materials

4.2.2 The $\mu$-rubbing process

4.2.3 Cell construction

4.2.4 Characterization

4.3 Results and discussion

4.3.1 Surface characterization

4.3.2 Stability of four-domain structure

4.3.3 Viewing angle characteristics

4.3.4 Contrast ratio and color properties

4.3.5 Heat aging

4.3.6 Optical simulations

4.3.6.1 Viewing angle and contrast ratio

4.4 Conclusions

Chapter 5 Four-Domain Twisted Vertically Aligned Liquid Crystal Display Using $\mu$-Rubbing

5.1 Introduction

5.2 Experimental

5.2.2 $\mu$-Rubbing setup 
$\begin{array}{lll}5.3 & \text { Results and discussion } & 101\end{array}$

$\begin{array}{ll}\text { 5.3.1 Surface characterization } & 101\end{array}$

5.3.2 Director orientations 103

5.3.3 Viewing angle and contrast ratio 105

$\begin{array}{ll}\text { 5.3.4 Electro-optical characterization } & 108\end{array}$

5.3.5 Line scanning: - Intensity profile 112

5.3.6 Chromaticity measurements 113

$\begin{array}{ll}\text { 5.3.7 Optical simulations } & 114\end{array}$

$\begin{array}{ll}\text { 5.3.7.1 Viewing angle and contrast ratio } & 114\end{array}$

$\begin{array}{ll}\text { 5.3.7.2 Director orientations } & 116\end{array}$

5.3.7.3 Disclination lines 119

$\begin{array}{ll}\text { 5.3.7.4 Electro-optical characteristics } & 121\end{array}$

$\begin{array}{lll}5.4 \text { Conclusions } & 122\end{array}$

$\begin{array}{llr}5.5 & \text { References } & 122\end{array}$

Chapter 6 Surface-Induced Pattern Formation in LC Mixtures

6.1 Introduction 125

6.2 Experimental 126

6.2.1 Materials 126

6.2.2 Friction deposition of PTFE 127

6.2.3 Cell construction $\quad 127$

$\begin{array}{ll}\text { 6.2.4 Characterization } & 128\end{array}$

$\begin{array}{lll}6.3 & \text { Results and discussion } & 128\end{array}$

6.3.1 Friction deposition of PTFE on glass substrates 128

6.3.1.1 Pretilt angle measurements 130

6.3.1.2 Electro-optical characterization 131

6.3.2 Alignment of E7 on patterned bi-polymer surfaces 132

6.3.2.1 PTFE-Homeotropic polyimide 132

6.3.2.2 PTFE-Planar polyimide 133 
$\begin{array}{ll}\text { 6.3.2.3 Electro-optical characterization } & 134\end{array}$

6.3.3 Phase diagram of LC mixtures (PEG-1000/E7) 136

6.3.4 Phase behavior of LC mixtures on bi-polymer surfaces 138

$\begin{array}{ll}\text { 6.3.4.1 PTFE-Homeotropic polyimide } & 138\end{array}$

$\begin{array}{ll}\text { 6.3.4.2 PTFE-Planar polyimide } & 140\end{array}$

$\begin{array}{ll}\text { 6.3.4.3 PTFE-PEG-SH surfaces } & 140\end{array}$

$\begin{array}{lll}6.4 \text { Conclusions } & 144\end{array}$

$\begin{array}{lll}6.5 & \text { References } & 144\end{array}$

$\begin{array}{ll}\text { Technology Assessment } & 147\end{array}$

Summary 151

$\begin{array}{ll}\text { Samenvatting } & 155\end{array}$

$\begin{array}{lr}\text { Publications } & 159\end{array}$

Acknowledgements $\quad 161$

$\begin{array}{ll}\text { Curriculum Vitae } & 163\end{array}$ 



\section{General Introduction}

\section{Chapter 1}

\subsection{Information display}

With the advent of the internet and the increase in portable electronics, the importance of information displays has increased tremendously, due to the need for fast transfer of large amounts of information for both business and entertainment purposes. Not only are computers used for business purposes, but more and more they are becoming centerpieces of the home entertainment system. It is the current interest of both research and business to improve the overall performance of display systems. Modern displays require a high accuracy, high resolution and high speed due to the rapid flow of information.

There is a wide variety of different types of visual displays commercially available or under development including liquid crystal displays (LCDs), ${ }^{1-5}$ cathode ray tubes (CRTs), ${ }^{6,7}$ plasma display panels (PDPs), ${ }^{8}$ field emission displays (FEDs), ${ }^{9-11}$ and organic, polymeric light emitting diodes (OLEDs, PLEDs). ${ }^{12,13}$ The CRT is currently the industry standard in spite of its high weight, bulky size, high power consumption and lack of esthetic appearance. The PDP can provide large-area, high-resolution images. PDPs use a glass vacuum envelope containing pixels comprising of small pockets of gas (Xe, $\mathrm{Ne})$ that are electrically excited to create a colored sub-pixel. Unfortunately, the price of these displays is quite high, and a high voltage is required to create the plasma. The FED possesses a back plane of many microscopic electron guns to provide electrons that directly impinge on a nearby phosphor anode to create images. Like CRTs, a high voltage is required to produce the electrons. Electro-luminescent displays like OLEDs and PLEDs are promising as emissive displays with a range of attractive features such as wide viewing angle and fast switching kinetics. These flat panel displays are based on electro-luminescent, conjugated, organic materials. At the moment, the lifetime, the 
daylight contrast and the battery life of OLEDs and PLEDs are low compared to LCDs, which is a limitation in most applications.

At the moment, the LCD is the dominant flat panel display type used in portable telephones, personal organizers, laptops, desktops and television because of its low energy consumption, brightness, viewing angle, flatness and lightweight. The LCD itself is, in fact, an incredible art of micro-engineering made reality by combining the disciplines of chemistry, physics, electronic engineering, process engineering and materials science.

\subsection{Liquid crystal display}

Large LCD-televisions are increasingly becoming a popular consumer product. Due to the advanced developments in operating principles and liquid crystal materials, LCDs are replacing conventional CRTs in a wide variety of places ranging from the computer monitors on office tables to the televisions in living rooms. Current research on LCDs is mainly focused on improving the image quality of the displayed image especially for moving images. Larger LCD screens require that even a single viewer seated at a comfortable viewing distance away from the screen is provided with a wide viewing cone of more than $40^{\circ}$ without gray-scale inversions in order to see uniform color from one extreme corner to another. The development of new enabling technologies addresses this problem.

There are different kinds of liquid crystal displays depending on their mode of operations. The commercially available LCDs include the following:

a. Twisted nematic liquid crystal display (TN-LCD)

b. Super twisted nematic display (STN)

c. Vertically aligned liquid crystal display (VA-LCD)

d. In-plane switching liquid crystal display (IPS-LCD) 
The TN mode in combination with passive matrix addressing is common in small displays including watches and calculators. The STN mode is used in small displays along with passive or active matrix addressing and is currently being used in mobile phones and personal organizers. Active matrix TN displays are often used in notebook computers and increasingly in high-end mobile applications where video processing is becoming standard. Today's high-end computer monitors and television screens based on LCDs use a VA or IPS mode of operation due to their outstanding performance with respect to switching speed, wide viewing angle, high contrast and good color quality. ${ }^{14}$ These different LCD modes are discussed more extensively in the following paragraphs.

\subsubsection{Twisted nematic display}

Among the many different operating modes in flat panel displays, the TN configuration holds an important position. ${ }^{15,16}$ In a TN-LCD, the liquid crystal director is twisted by an angle of $90^{\circ}$, and the molecules act as a wave guide, guiding the electrical field vector of polarized light. Figure 1.1 shows a schematic diagram of a typical TN-LCD.

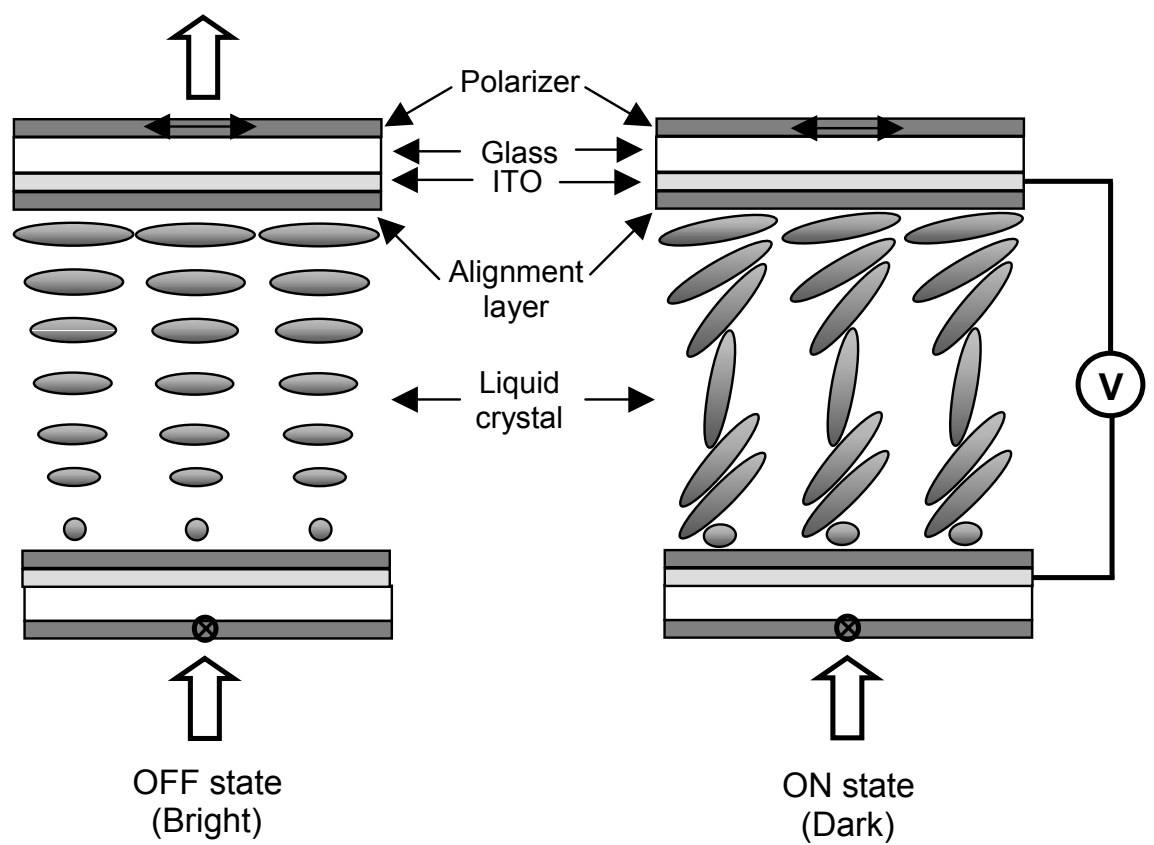

Figure 1.1: Schematic representation of a twisted nematic liquid crystal display in the OFF and ON state. 
A liquid crystal (LC) material is sandwiched between two glass substrates coated with transparent indium tin oxide (ITO) electrodes and a rubbed polyimide alignment layer. The rubbing directions of the top and bottom substrates are oriented orthogonal to each other creating a $90^{\circ}$ twist of the LC molecules. A small amount of chiral dopant is added to the liquid crystal to control the sense of the twist. The two glass substrates are kept at a small distance $(4-6 \mu \mathrm{m})$ from each other depending on the LC material. The polarizer is aligned so that the transmission axis is parallel to the director at the input end of the LC (E-mode operation). The analyzer is aligned so that the transmission axis is parallel to the director at the exit end of the liquid crystal. The polarized light coming from the first polarizer will pass through the LC medium, where it is twisted due to the waveguiding of the LC and it passes through the analyzer creating a bright state (offstate). This configuration is usually called the normally white mode (as opposed to the normally black mode in which the polarizers are placed in a parallel position). Upon application of an electric field (3-5 V) to the electrodes, the waveguiding nature of the LC medium is lost. ${ }^{17}$ This is due to the dielectric anisotropy of the LC molecules; the molecules align parallel to the direction of the applied electric field. In this homeotropically aligned state, the polarized light from the first polarizer remains unchanged and is blocked by the analyzer, which is orthogonal to the polarizer creating a black state (on-state). By controlling the voltage, it is possible to control the transmission of light passing through the cell and to control gray scales. Integration of the LC cell with color filters and electronic driving circuits enables the manufacturing of high quality, full-color displays. As can be seen in Figure 1.1 the addressed liquid crystal does not acquire the fully homeotropic state. In general this is not a problem because of the so-called self-compensation principle. Each molecule in the lower part of the cell has an orthogonal equivalent in the upper part that compensates optically. Only at off-axis angles this might provide a viewing angle problem as will be discussed later. 


\subsubsection{Super twisted nematic display}

In early 1980 's, the super twisted nematic liquid crystal display (STN-LCD), which has a twist of over $180^{\circ}$, typically between $210^{\circ}$ and $270^{\circ}$, was discovered. ${ }^{18-20}$ Figure 1.2 shows the basic principle of an STN cell. The cell structure of an STN-LCD is similar to that of a TN-LCD.

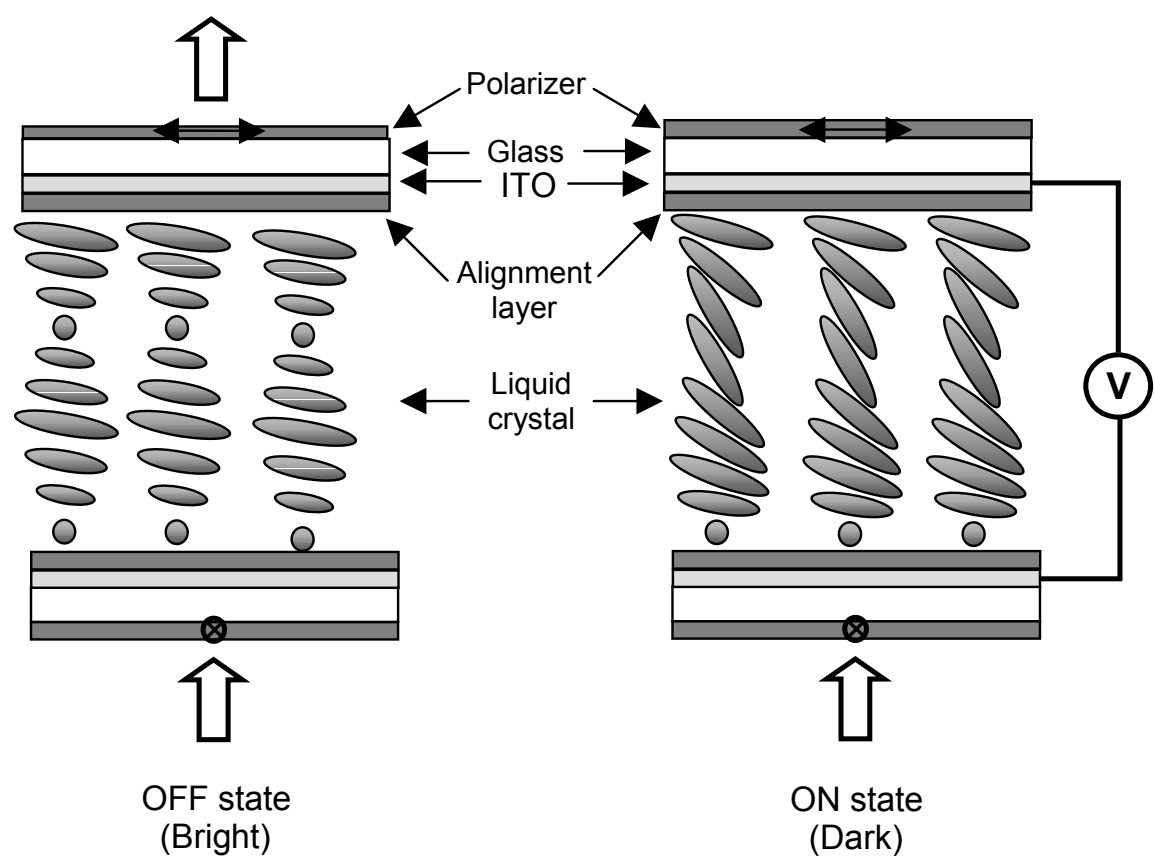

Figure 1.2: Setup and basic principle of the super twisted nematic display in the OFF and $O N$ state.

The liquid crystal in the cell has either a right handed or a left handed $270^{\circ}$ twist. Both will satisfy the boundary conditions determined by the rubbing directions. A high volume fraction of a chiral dopant with a high intrinsic optical rotatory power is added to maintain the high twist angles $\left(>90^{\circ}\right) .^{17}$ The handedness of the dopant molecules imparts a macroscopic twist to the whole nematic structure. The super twisted configuration possesses a steep transmission-voltage curve, which makes it accessible for multiplexing by electrode arrays at the top and bottom plate (passive matrix addressing). This is achieved at the expense of the response time, which is slow in comparison to TN- 
LCDs, about 200 milliseconds as opposed to about 60 milliseconds. STN displays are not as bright and tend to give blue and yellow images, rather than black and white ones. It was discovered that placing a second layer of STN-LCs above the first, with the STN chains twisted in the opposite direction improves this i.e. a display is produced with true black and white images. This type of display with the double layer of STN-LCs is aptly called the double super twisted nematic LCD or DSTN-LCD. These displays are, obviously, much thicker, heavier and more expensive to manufacture.

\subsubsection{Vertically aligned display}

Commercially available large area flat panel displays with wide viewing angles are often based on the vertically aligned (VA) configuration. ${ }^{21,22}$ The VA mode was introduced in $1971^{23}$ and optimized in 1998 by Fujitsu. Figure 1.3 shows the operating principle of a typical VA-LCD. In VA systems, the liquid crystal molecules are aligned perpendicular (homeotropic) to the substrate at zero fields. Therefore, this system requires LC mixtures with negative dielectric anisotropy $(\Delta \varepsilon<0)$ because the molecules switch from a homeotropic state to a planar state in an electric field.

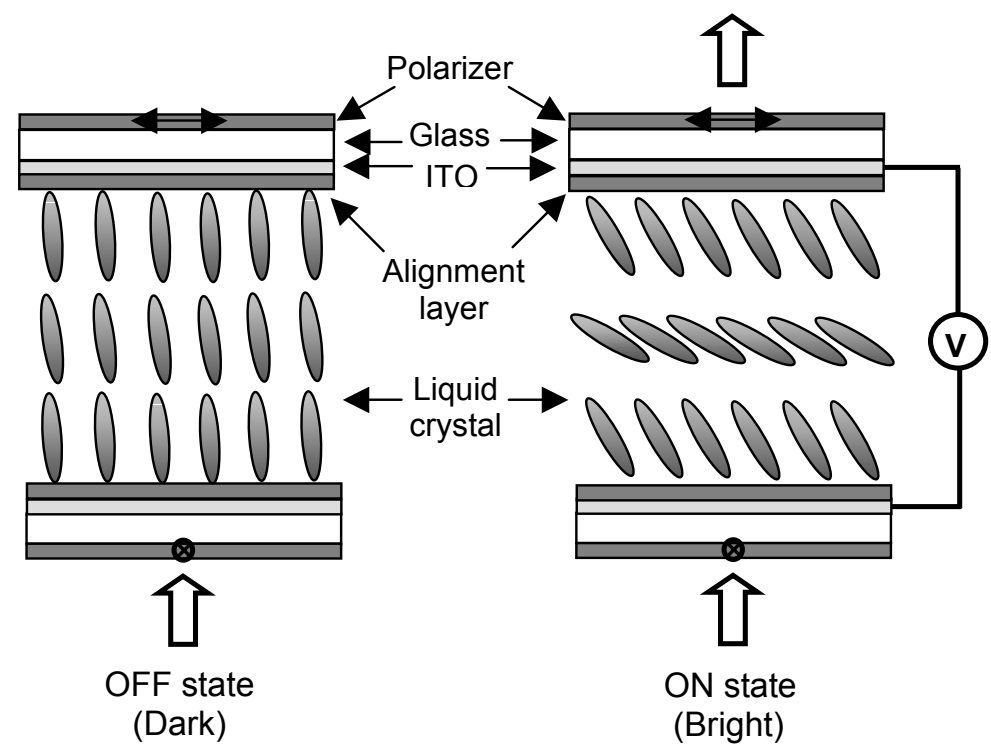

Figure 1.3: Basic principles of VA switching mode in the OFF and ON state. 
Since all of the LC molecules are aligned normal to the substrate, the polarized light passes through the cell without a change in polarization by the LC molecules and the light is completely blocked by the analyzer creating a perfectly black state. In the onstate, the LC molecules rotate to a horizontal position, creating a white state. Compared to the twisted nematic configuration, VA systems can achieve higher switching speeds because there is no twisted structure and the LC molecules are switched between vertical and horizontal positions. Due to the perfect black state in the off-state the VA mode also provides good contrast. The optical retardation that builds up at off-axis angles can be easily compensated by a simple c-plate retarder foil. A switching time of $25 \mathrm{~ms}$ was achieved in the first generation VA displays. The right choice of cell gap and liquid crystal further improves the switching time. Currently, negative $\Delta \varepsilon$ LC mixtures used for VA-LCDs are based on the 1,2-difluorobenzene building blocks. It was found that the addition of fluorine atoms in the chemical structure would increase the value of dielectric constant $(\Delta \varepsilon)$ and increase the switching speed. ${ }^{24}$

\subsubsection{In - plane switching mode}

In 1995, Hitachi developed a new mode of liquid crystal display operation in order to fabricate a flat panel display with an improved viewing angle and high contrast ratio. ${ }^{17,25-28}$ In the case of in-plane switching (IPS) cells the electrodes are placed only on one glass substrate. In this mode of operation, the electric field is applied through inter-digital electrodes, which are kept parallel to each other on the lower substrate of the display. ${ }^{29}$ The molecules are switched in the plane of the substrate, leading to significantly reduced viewing angle dependency of the transmitted light intensity, in comparison to a TN-display. Figure 1.4 shows the operating principle of an LCD using the IPS mode of operation. In the off-state the optical axis direction of the uniformly aligned liquid crystals is parallel to one of the polarization axis of two crossed polarizers and, consequently the off-state is black. 
In an applied field, the optical axis of the liquid crystals gradually deviates from the polarization axis. The transmission of light increases because of the phase retardation due to the different propagating speeds of the extraordinary and ordinary rays in the liquid crystal medium.

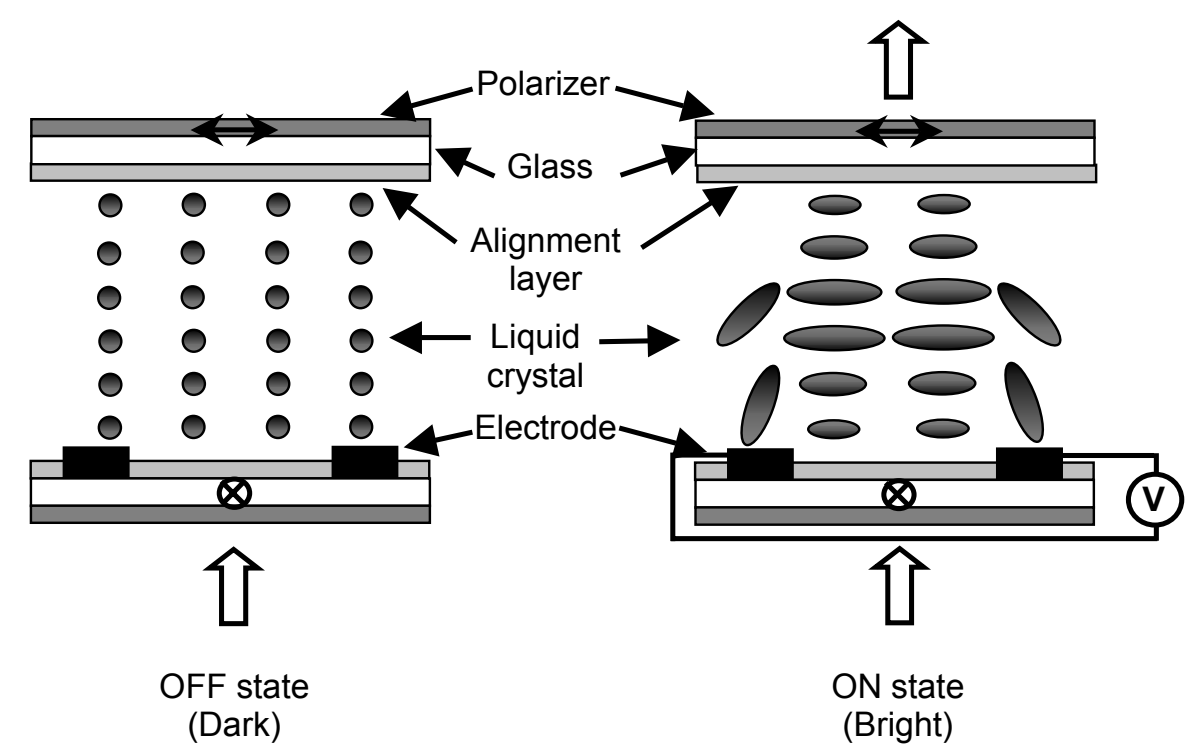

Figure 1.4: Working principle of the in-plane switching liquid crystal display in the OFF and ON state.

The response time of the IPS mode depends on the cell gap, rotational viscosity of liquid crystal, twist elastic constant, applied electric field and the rubbing angle. By increasing the distance between the inter-digital electrodes one can increase the aperture ratio, but this may lead to an increase in the switching voltage. The increase in switching voltage is usually compensated by using LCs with higher dielectric anisotropy $(\Delta \varepsilon)$ but that also leads to higher rotational viscosities, which will affect the switching time. ${ }^{24} \mathrm{~A}$ proper selection of LC, inter-digital electrode spacing and the cell gap improves the properties of the display. 


\subsection{Viewing angle of LCDs}

One of the major drawbacks of LCDs is its viewing angle dependency. This viewing angle problem arises due to the inherent birefringence of liquid crystal material; different viewing directions give varying degrees of birefringence, resulting in the optical transmission behavior being strongly dependent on the viewing direction. ${ }^{30}$ So the highquality images are restricted to a limited viewing cone. This limited viewing cone needs to be broadened since LCDs used in avionics, wide-screen displays, and elsewhere require broad viewing angle characteristics. The reason for viewing angle dependency is explained in Fig. 1.5 with a structure of typical TN-LCD, in which the tilted middle director causes different phase retardation at oblique angles creating an optical anisotropy.

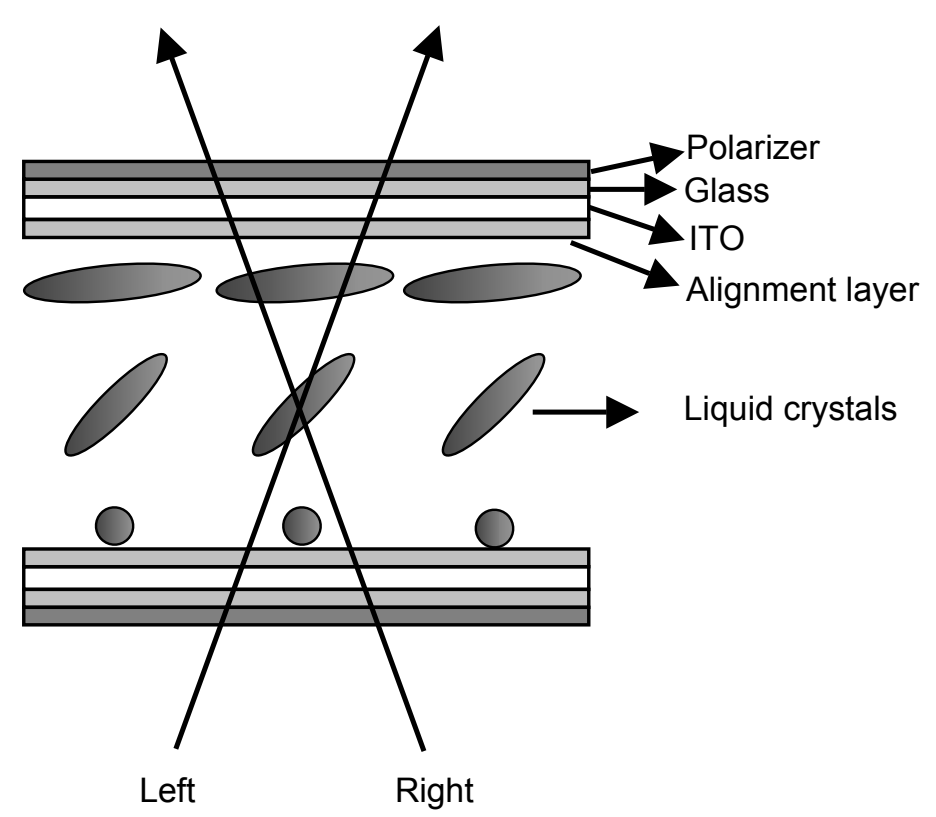

Figure 1.5: Structure of a simple TN-LCD cell. The electric field is in the longitudinal direction. The tilted mid-plane LC directors cause different phase retardation upon viewing from right or left oblique angles.

There are two kinds of viewing angle problems: 1) contrast loss due to a rest retardation at oblique angles and 2) gray-scale inversion due to the fact that at oblique angles the transmission-voltage curve exhibits a minimum. There are two different 
approaches used commercially to improve the viewing angle problems of the LCDs. The first one is referred to as the external approach, and uses suitable optical compensation films out side the cell structure to enhance the viewing angle. This can compensate for the contrast loss and may alleviate the gray-scale inversion (though not compensate it completely). In the second approach (the internal approach) inside of the electro-optical cell is modified and subdivided into different domains to compensate for the birefringence of the LC.

\subsubsection{Optical compensation films}

One of the most commonly used methods to improve the viewing angle is to use optical compensation films. ${ }^{30-32}$ In this method of optical birefringence compensation, a thin film of birefringent material is placed onto a LCD at a proper position to neutralize the angular dependence. The choice of the optical birefringence film depends on the mode of operation.

Fuji developed compensation films based on discotic materials for LCDs. ${ }^{33-36}$ Discotic monomers were spin-coated or rolled over polyimide surface. At the air-film interface, the discotic layer is splayed naturally at an angle of $40^{\circ}-68^{\circ}$. The molecular tilt angle continuously evolves within film. The orientation of the discotic liquid crystals is fixed by UV polymerization. These films exhibit a unique splayed structure, an asymmetric angular-dependence phase retardation and negative birefringence, and it is particularly suitable for compensating the viewing angle of a TN cells. Unlike a spincoated negative birefringence film that has symmetric angular phase retardation, the angular phase retardation of a discotic film is asymmetric. A TN display provided with a tilted discotic film on each side between the glass cell and the polarizer film has a viewing angle up to $\sim \pm 60^{\circ}$. Good phase compensation is obtained for all three primary colors and a high contrast ratio is generated. Besides contrast improvement these tilted discotic films also improve the display on gray scale inversion at large off-normal.

In the case of STN-LCDs, stacks of films of positive birefringence can be used for phase retardation compensation in the field off-state. ${ }^{37}$ These plates must be placed 
between the polarizer and the analyzer. This significantly reduces the leakage of light in the dark state over an extended field of view leading to an improved contrast ratio and a reduced color desaturation. In the ideal case, films are used that have the same birefringence profile as the electro-optical cell but with an opposite handedness. Layers built from chiral-doped calamitic liquid crystal polymers are most suited for this purpose. $^{38}$

In VA-LCDs, films with a negative birefringence are employed to improve the viewing angle characteristics. In the case of VA-LCDs at the field off-state, the phase retardation due to positive birefringence of the $\mathrm{LC}$ cell is compensated by a thin film of negative birefringence. ${ }^{30}$ This leads to a net phase retardation of near zero for virtually all angles of incidence. In the case of IPS mode the optical compensation is performed using a single biaxial film or two uniaxial films. ${ }^{39,40}$

\subsubsection{Multidomain liquid crystal display}

The internal approach to improve the viewing angle problems of LCDs is based on dividing each pixel into two or four sub-pixels with different director orientations to average the viewing angle dependency. Using this multidomain concept one can also avoid prevailing limitations such as contrast deterioration, color shift and gray scale inversion. In Fig.1.6 a schematic drawing is presented in which the gray-scale performance of a single domain and multidomain display is compared. By dividing a single pixel into two-domain or four-domain structure an averaging effect of LC alignment is obtained which improves gray-scale performance. 


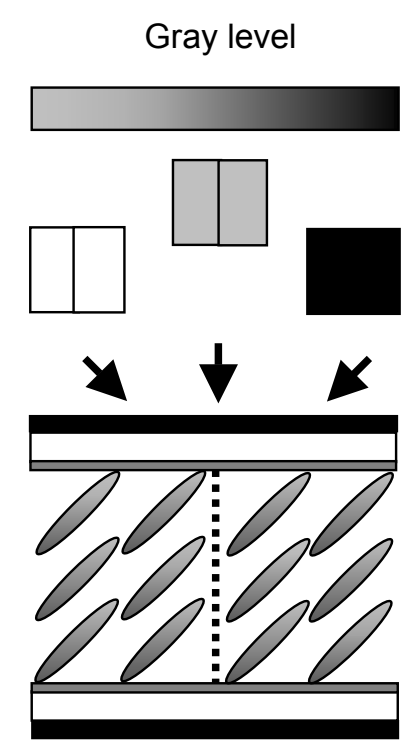

Single domain

\section{Gray level}

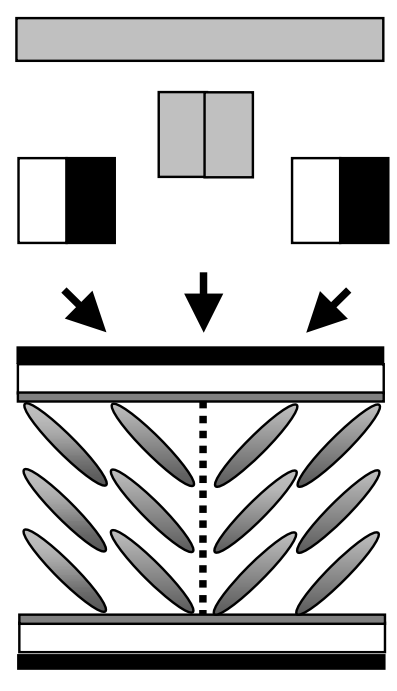

Multidomain

Figure 1.6: Comparison of gray-scale display performance of single and multidomain LCDs.

There are different techniques available to fabricate multidomain structures which include photoalignment methods, ${ }^{41,42}$ reverse rubbing ${ }^{43}$ and $\mathrm{SiO}_{2}$ oblique evaporation. ${ }^{44}$ For instance, the photoalignment method for creating multidomain structure utilizes photopolymerisable materials like cinnamates and coumarins in combination with photo masks and linearly polarized light to create different alignments. These materials undergo a crosslinking reaction when exposed to high energy UV light. When exposed with polarized light only those chromophores react that are aligned with their dipole moment parallel to the electrical field vector of the light. This selectivity creates an anisotropy in the surface of these polymer layers that affects the liquid crystal alignment. ${ }^{45}$ None of the above described methods for generating multidomain displays are used commercially, which is related to a poor control of the surface pretilt angle, image retention phenomena and/or complex fabrication routes. ${ }^{46,47}$

There are different methods used to create multidomain structures in VA-LCDs, which include the fabrication of ridges or protrusions on the substrates. ${ }^{48-50}$ These protrusions provide a small local off-axis alignment of the liquid crystal molecules that 
determine a defined switching direction for each domain. Under an applied voltage, the oblique electrical fields around the ridges assist the LC molecules to incline to the respective direction as shown in Fig. 1.7.

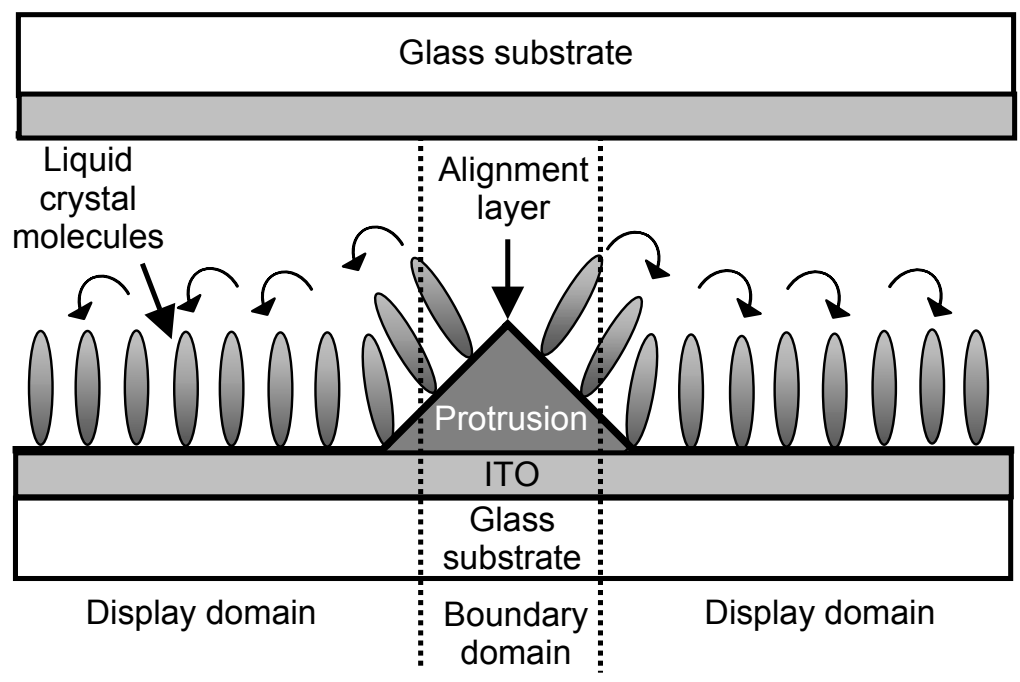

Figure 1.7: Alignment of liquid crystals on protrusions in a multidomain VA-LCD.

Photolithography is usually used to create these kinds of protrusions. Due to the presence of these protrusions, disclination lines are formed between the domains that affect the contrast of the display. Usually these disclination lines are covered by a light absorbing layer (black matrix) and consequently they do not reduce the contrast. Fourdomain liquid crystal alignment can be achieved by providing both the top and the bottom substrate surfaces with protrusions that have a mutual orthogonal orientation. The viewing angle characteristics of commercially available four-domain VA-LCDs are symmetrical, and the viewing angles in the vertical and horizontal directions are $160^{\circ}$ or more. Gray-scale inversion and the dependency of chromaticity on the viewing angle are virtually absent in these displays.

As was discussed in Section 1.3.1, a common problem of all vertically aligned displays, independent of mode, is that there is significant light leakage in the zero voltage state at larger viewing angles. Simple $c$-plate compensation can be used to minimize the 
light leakage and improve the viewing volume in the zero voltage state. ${ }^{30}$ Also, the combination of an optical compensation film with a multidomain structure within a cell can give a synergistic effect with respect to the display performance.

In the previous paragraphs different techniques were described to improve the viewing angle and gray-scale inversion in a wide variety of LCDs. Typically, compensation films are very expensive and also increase the thickness of the display. On the other hand the currently used techniques to fabricate multidomain pixels are process intensive and expensive. Therefore a need persists for new techniques to fabricate mutlidomain structure for viewing angle compensation which circumvent the above described disadvantages.

\subsection{Objectives}

The main objective of this study is to develop new methods for the fabrication of multidomain structures in LCDs to improve their viewing angle characteristics. More specifically, the currently used methods are laborious and exotic materials are required, which increases the displays cost. Therefore, a microrubbing ( $\mu$-rubbing) process is investigated and applied to the twisted nematic and vertically aligned modes to generate multidomain configurations. The prime objective is to develop methods that are simple, economical and compatible with currently used LCD production routes. Moreover, it is also attempted to use the developed techniques for other applications such as switchable gratings and security features.

\subsection{Scope of the thesis}

In Chapter 2 the $\mu$-rubbing process is described which is used to create micropatterns on both unrubbed and pre-rubbed polyimide orientation layers. The liquid crystal alignment originating from the $\mu$-rubbing is investigated and discussed. The electro-optical characteristics and pretilt of the liquid crystals are also discussed. The director orientations, the formation of disclination lines and electro-optical characterizations are numerically calculated and compared with the experimental 
observations. Moreover, an attempt was made to create a multidomain TN display and to evaluate its properties.

Chapter 3 focuses on the $\mu$-rubbing of homeotropic polyimide to create a planar alignment with a high pretilt angle. The variation of pretilt angle and switching characteristics with applied load are described. The director orientation and the formation of disclination lines are discussed based on experiments and optical simulations.

Chapter 4 describes the fabrication of four-domain TN cells using the $\mu$-rubbing process in combination with a homeotropic alignment layer. The stability of the fourdomain structure is described in terms of the required pretilt angle in zero fields. Theoretical simulations of the formation of disclination lines in the structure are compared with experimental data. The viewing angle characteristics, contrast ratio, electro-optical parameters of the multidomain TN cell are presented.

In Chapter 5 the $\mu$-rubbing process is used to generate micro-patterned alignment layers with a very high pretilt $\left(>80^{\circ}\right)$ using homeotropic polyimide. Two different display modes i.e. twisted and frustrated twisted vertically aligned mode are described. The electro-optical characteristics, viewing angle characteristics and color measurements are presented and compared with the optical simulation results.

In Chapter 6 the patterned alignment of liquid crystals on surface tension patterns is described. The friction deposition of polytetrafluoroethylene (PTFE) on homeotropic polyimide and planar polyimide is investigated with respect to the orientation of LCs. The pretilt angle variation with applied load and switching characteristics are also evaluated. The demixing of LC mixtures on surface with difference in polarity is also studied.

Finally the $\mu$-rubbing process and its ability to create different kinds of alignment on the micron scale is discussed in the Technology Assessment with an emphasis on performance and applications in the display field. Additionally, the use of $\mu$-rubbing in other applications (switchable gratings, security) is discussed. 


\subsection{References}

[1] S. Musa, Sci. Am., 277, 87 (1997).

[2] M. Schadt, W. J. Helfrich, Appl. Phys. Lett. 18, 127 (1971).

[3] N. A. J. M. van Aerle, J. Soc. Inf. Display 2, 41 (1994).

[4] J. M. Geary, J. W. Goodby, A. R. Kmetz, J. S. Patel, J. Appl. Phys. 62,10 (1987).

[5] S. W. Depp, W. E. Howard, Sci. Am. 266, 40 (1993).

[6] S. Sherr, Electronic displays, $2^{\text {nd }}$ edition, Wiley Interscience, New York (1993).

[7] J. C Whitaker, Electronic displays: technology, design and applications. McGrawHill, New York (1994).

[8] H. S. Nalwa, L. S. Rohwer, Handbook of Luminescence, Display Materials and Devices, Display Devices, American Scientific Publishers, Chapter 3 (2003).

[9] W. H. Heer, A. Chatelain, D. Ugarte, Science 270, 1179 (1995).

[10] Q. H. Wang, A. A. Setlur, J. M. Lauerhass, J. Y. Dai, E. W. Seeling, R. P. H. Chang, Appl. Phys. Lett. 72, 2912 (1998).

[11] A. P. Burden, Inter. Mater. Rev. 46, 213 (2001).

[12] H. S. Nalwa, L. S. Rohwer, Handbook of Luminescence, Display Materials and Devices, Organic Light-Emitting Diodes, American Scientific Publishers, (2003).

[13] J. H. Burroughes, D. D. C. Bradley, A. R. Brown, R. N. Marks, R. H. Friend, P. L. Burns, A. B. Holmes, Nature 374, 539 (1990).

[14] Y. Ishii, S. Mizushima, M. Hijikigawa, SID’01 Digest, 1090 (2001).

[15] M. Schadt, W. Helfrich, Appl. Phys. Lett. 18, 127 (1971).

[16] J. A. Castellano, Mol. Cryst. Liq. Cryst. 165, 389 (1988).

[17] P. Yeh, C. Gu, Optics of Liquid Crystal Displays, John Wiley \& Sons, Inc. New York Chapter 5 (1999).

[18] T. J. Scheffer, J. Nehring, Appl. Phys. Lett. 36, 1021 (1984).

[19] H. Fukuro, S. Kobayashi, Mol. Cryst. Liq. Cryst. 163, 157 (1988). 
[20] T. J. Scheffer, J. Nehring, Liquid Crystals: Applications and Uses, Vol. 1, World Scientific, Singapore, Chapter 10 (1990).

[21] J. Y. Hwang, K. J. Lee, D. S. Seo, T. H. Kim, Jpn. J. Appl. Phys. 42, L672 (2003).

[22] Y. Koike, K. Okamoto, FUJITSU Sci. Tech. J. 35, 221 (1999).

[23] M.F. Schiekel, K. Fahrenson, Appl. Phys. Lett. 19, 391 (1971).

[24] D. Pauluth, K. Tarumi, J. Mater. Chem. 14, 1219 (2004).

[25] R. A. Soref, J. Appl. Phys. 45, 5466 (1974).

[26] M. Oh-e and K. Kondo, Appl. Phys. Lett. 67, 3895 (1995).

[27] M. Oh-e and K. Kondo, Appl. Phys. Lett. 69, 623 (1996).

[28] R. Kiefer, B. Weber, F. Windscheid, G. Baur, Japan Display'92 Digest, 547 (1992).

[29] M. Oh-e, M. Ohta, S. Aratani, K. Kondo, Asia Display’ 95, 577 (1995).

[30] P. Yeh, C. Gu, Optics of Liquid Crystal Displays, John Wiley \& Sons, Inc. New York Chapter 9 (1999).

[31] N. Yamagishi, H. Watanabe, K. Yokoyama, SID’89 Digest, 316 (1989).

[32] Y. Yamagnchi, T. Miyashita, T. Uchida, SID’93 Digest, 277 (1993).

[33] H. Mori, Y. Itoh, Y. Nishiura, Y. Shinagawa, IDW'96 Digest, 189 (1996).

[34] H. Mori, Y. Itoh, Y. Nishiura, Y. Shinagawa, Jpn. J. Appl. Phys. 36, 143 (1997).

[35] H. Mori, Jpn. J. Appl. Phys. 36, 1068 (1997).

[36] H. Mori, E. Aminaka, Y. Itoh, Y. Shinagawa, IDW’01, 593 (2001).

[37] J. C. Kim, T. Watanabe, S. Miyata, Jpn. J. Appl. Phys. 36, 232 (1997).

[38] I. Heynderickx, D.J. Broer, Mol. Cryst. Liq. Cryst. 203, 113 (1991).

[39] Y. Saitoh, S. Kimura, K. Kusafuka, H. Shimizu, Jpn. J. Appl. Phys. 37, 4822 (1998).

[40] J. E. Anderson, P. J. Bos, Jpn. J. Appl. Phys. 39, 6388 (2000). 
[41] M. Schadt, H. Seiberle and A. Schuster, Nature 381, 212 (1996).

[42] Schadt, M., Helfrich, W. Appl. Phys. Lett. 18, 127 1971).

[43] J. Chen, P. J. Bos, D. L. Johnson, D. R. Bryant, J. Li, S. H. Jamal, J. R. Kelly, J. Appl. Phys. 80, 1985 (1996).

[44] J. Li, E. S. Lee, H. Vithana, P. J. Bos, Jpn. J. Appl. Phys. 35, L1446 (1996).

[45] M. Schadt, K. Schmitt, V. Kozinkov, V. Chigrinov, Jpn. J. Appl. Phys. 31, 2155 (1992).

[46] M. O’Neill, S. M. Kelly, J. Phys. D: Appl. Phys. 33, R67 (2000).

[47] A. Lien, C. Chen, H. Inoue, Y. Saitoh, SID’97 Digest, 203 (1997).

[48] J. Y. Hwang, K. J. Lee, D. S. Seo, T. H. Kim, Jpn. J. Appl. Phys. 42, L672 (2003).

[49] Y. Koike, K. Okamoto, FUJITSU Sci. Tech. J. 35, 221 (1999).

[50] S. C. A. Lien, C. Cai, R. W. Nunes, R. A. John, E. A. Galligan, E. Colgan, J. S. Wilson, Jpn. J. Appl. Phys. 37, L597 (1998). 


\section{Chapter 2}

\section{Patterned Alignment of Liquid Crystals by $\mu$-Rubbing*}

\subsection{Introduction}

As discussed in Chapter 1, alignment layers are extensively used in the production of liquid crystal displays (LCDs) to orient the liquid crystal molecule to give a desired optical effect. ${ }^{1-6}$ For instance, planar alignment of liquid crystals (long axes of molecules in plane of substrate) is often achieved through a combination of specific polyimide layers and mechanical rubbing (buffing) procedure, producing unidirectional alignment that propagates over macroscopic distances. Polyimides are usually preferred because of their excellent properties with respect to chemical resistance, thermal stability, adhesion to substrates, high resistivity and transparency in the visible spectrum. The mechanical rubbing of polyimides is performed with a velvet type synthetic cloth in direct contact with the polyimide.

Recently there have been a variety of non-contact routes for patterning alignment layers in LCDs. The patterned alignment of cinnamates, coumarins and special polyimide coated surfaces was extensively investigated using lithographic techniques in combination with linearly polarized light. ${ }^{7-10}$ Direct writing with low-energy ion-beams on amorphous, inorganic thin films was also used as a suitable non-contact method for patterned alignment. ${ }^{11}$ Patterned alignment of liquid crystals on printed self-assembled monolayers was also explored by a number of groups. ${ }^{12-14}$ At the moment, none of the above mentioned techniques are used extensively in production of LCDs predominantly due to their poor control of surface pretilt angle, image retention phenomena, and/or complex fabrication routes.

*This chapter is partly reproduced from: S. Varghese, S. Narayanankutty, G. P. Crawford, C. W. M. Bastiaansen, D. J. Broer, Adv. Mater. 18, 1600 (2004). 
More recently, the micro-patterning of alignment layers was also performed with an extremely sharp stylus by Rosenblatt. ${ }^{15,16}$ Also, Yokoyama ${ }^{17}$ has used micropatterning of surfaces with a sharp stylus to create a tristable nematic liquid crystal device. The use of direct writing techniques with a sharp stylus imposes severe limitations on the production of alignment layers for large area displays (i.e. the typical width of a single recorded line is less than $100 \mathrm{~nm}$ and consequently lengthy recording times are required to produce a single pixel or, more importantly, a large area substrate). Therefore, a need persists for new methods to produce patterned, well defined alignment layers which are compatible with large scale production techniques.

In this chapter a simple and efficient tool for patterning alignment layers is presented based on the rubbing of polyimides with a macroscopic, metallic object. The process is coined as microrubbing ( $\mu$-rubbing) process. The different types of LC alignment are characterized and compared with optical simulations. The prime objective is to explore new routes for the micro-patterning of alignment layers for the fabrication of multidomain LCDs and security devices.

\subsection{Optical simulations:- Theory}

There are different numerical methods available for the calculation of director profiles, viewing angle characteristics and electro-optical behavior of the LCDs. Simulation methods for director profiles are derived from the Oseen-Frank free energy equation for the electric and elastic energies of the liquid crystal given by the following expression. ${ }^{18}$

$$
\mathrm{F}=\frac{1}{2} \int_{v}\left\{k_{11}(\nabla \cdot \bar{n})^{2}+k_{22}(\bar{n} \cdot \nabla \times \bar{n})^{2}+k_{33}(\bar{n} \times \nabla \times \bar{n})^{2}-\varepsilon_{0} \Delta \varepsilon(\overline{\mathrm{E}} \cdot \bar{n})^{2}\right\} d v
$$

Where $k_{11}, k_{22}$ and $k_{33}$ are the splay, twist and bend elastic constants, respectively; $\Delta \varepsilon$ is the dielectric anisotropy, $\bar{n}$ is the nematic director, $\overline{\mathrm{E}}$ is the applied electric field, and $\varepsilon_{0}$ is the permittivity of free space and $v$ is the volume of the liquid crystal. Vector representation methods are usually used in most simulation algorithms. Minimizing this expression, and using appropriate boundary conditions the director profile can be derived. 
There are two ways to solve the resulting equations with numerical calculations, the finite difference method (FDM) and the finite element method (FEM). The two dimensional simulations of director profiles in this thesis were made with the 2dimMOS software package using a vector representation and the FEM with a mesh of triangular elements. ${ }^{19,20}$

For the calculation of the optical transmission of an LCD cell the extended Jones matrix method ${ }^{21}$ was used, which takes in to account single reflection at each surface but neglects multiple reflections and their interference. This method provides a systematic way to analyze the propagation of monochromatic plane waves in a layered birefringent medium.

\subsection{Experimental}

\subsubsection{Materials}

Indium tin oxide (ITO) coated glass substrates were purchased from Merck. The polyimide precursor Al1051 was used as the planar orientation layer (JSR electronics). Spin coating of polyimide precursor was performed using a Karl Süss CT 62 spin coater ( $5 \mathrm{~s}$ at $1000 \mathrm{rpm}, 40 \mathrm{~s}$ at $5000 \mathrm{rpm}$ ) on the ITO side of $2.5 \times 2.5 \mathrm{~cm}$ glass/ITO substrates. After spin coating, the substrates were preheated to $100{ }^{\circ} \mathrm{C}$ for 10 minutes. The samples were then imidized at $180{ }^{\circ} \mathrm{C}$ for 90 minutes in a vacuum oven. The thickness of the polyimide coating was $100 \mathrm{~nm}$. A liquid crystal (E7), a multi component cyanobiphenyl and terphenyl mixtures $\left(T_{N / I}=58{ }^{\circ} \mathrm{C}, \rho=1.06 \mathrm{~g} / \mathrm{cm}^{3}, \varepsilon_{/ /}=19\right.$ and $\varepsilon_{\perp}=5.2$, and $\Delta n=$ 0.225), was used as obtained from Merck (Darmstadt, Germany).

\subsection{2 $\mu$-Rubbing setup}

The $\mu$-rubbing of unrubbed and pre-rubbed (buffed) polyimide was carried out at room temperature. The pre-rubbing was performed by uniaxially buffing the polyimide using a velvet cloth to get a uniform alignment. The $\mu$-rubbing of pre-rubbed polyimide was 
carried out perpendicular to the rubbing direction with a mechanical device having a metallic sphere of $1 \mathrm{~mm}$ in diameter under different loads. Patterns were recorded by writing at a constant load and velocity $(10 \mathrm{~mm} /$ minute). Figure 2.1 depicts the experimental setup used in the $\mu$-rubbing process. The twisted nematic $(\mathrm{TN})$ area created by the $\mu$-rubbing process is too small $\left(28 \mu^{2}\right)$ to perform accurate electro-optical and pretilt measurements. To overcome this problem the metallic sphere was replaced by a $500 \mu \mathrm{m}$ wide well polished metallic ring with chamfered edges (see Fig. 2.1b). By implementing this process, patterns with larger line width were generated, analogous to the metallic sphere method. The edges are carefully aligned with the rubbed regions so they would coincide with one another to achieve uniformity of the $\mu$-rubbing region over a large area.

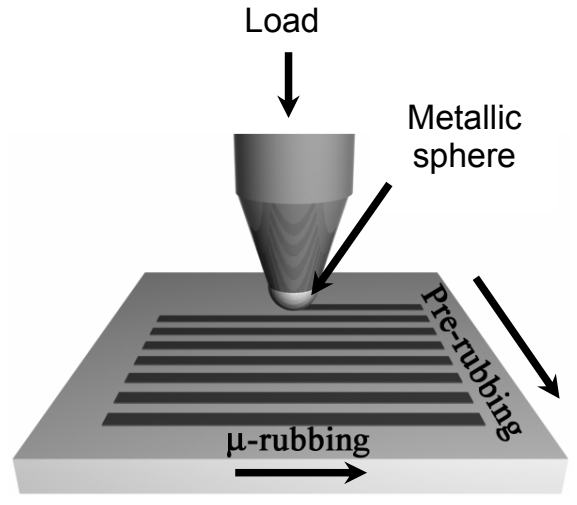

(a)

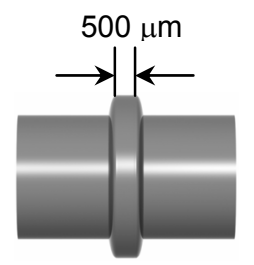

(b)

Figure 2.1: The $\mu$-rubbing setup, pre-rubbing and $\mu$-rubbing directions are depicted in the picture (a) and the metallic ring used (b).

\subsubsection{Construction of cell}

Electro-optical cells were constructed using ITO coated glass with the polyimide film. The $\mu$-rubbing direction on the top and bottom surface oriented orthogonally and secured with UV curable glue (Norland UV Sealant 91) having $18 \mu \mathrm{m}$ spacers. The cell was 
capillary filled at $80{ }^{\circ} \mathrm{C}$ with the liquid crystal material E7, approximately $20{ }^{\circ} \mathrm{C}$ above the nematic-isotropic transition temperature of the liquid crystal.

\subsubsection{Characterization}

The $\mu$-rubbed patterns were investigated by atomic force microscopy (AFM, Nanoscope IIIa, Digital Instruments, Santa Barbara, California) equipped with conventional $\mathrm{Si}_{3} \mathrm{~N}_{4}$ cantilever and tip in tapping mode. Optical micrographs were recorded with polarized light microscopy (Zeiss LM Axioplan) equipped with a digital camera. To obtain line scans a Labview computer program was used to draw a line on the optical microscopy images and the intensity vs. position was plotted. The surface morphology was also investigated by using environmental scanning electron microscopy (ESEM, Philips XL30 FEG operated at 1-10 kV). The cell thickness was determined by UV-visible spectroscopy (Shimadzu UV-3102 PC).

\subsubsection{Electro-optical characterization}

The electro-optical characteristics were investigated using DMS 703 display measuring system (Autronic-Melchers $\mathrm{GmbH}$ ). A square wave was used to drive the cells for the dynamic response measurements.

\subsubsection{Pretilt angle measurement}

Pretilt measurements were carried out using an Autronic TBA 107 instrument that employs the crystal rotation method. Antiparallel cells with a thickness of $18 \mu \mathrm{m}$ were constructed for this purpose. Figure 2.2 shows a schematic diagram of the setup. A homogeneously oriented uniaxial and anti-parallel sample is rotated over an angle $\varphi$ with respect to a He-Ne laser beam. The light is polarized at $45^{\circ}$ with the rotation axis. The liquid crystal director is perpendicular to the rotation axis. The birefringent sample introduces an optical phase difference ' $\delta$ ', which is given by the following equation. 


$$
\delta=\frac{2 \pi d}{\lambda}\left[\frac{n_{0}^{2}-n_{e}^{2}}{n^{2}} \sin \theta \cos \theta \sin \varphi+\frac{n_{0} n_{e}}{n^{2}} \sqrt{n^{2}-\sin ^{2} \varphi}-\sqrt{n_{0}^{2}-\sin ^{2} \varphi}\right]
$$

Where $n_{0}, n_{e}$ represents ordinary and extraordinary refractive index, $\varphi$ is the azimuthal angle. This leads to an elliptical polarization, which is converted to linear polarization using quarter wave plate. This resulting polarization is rotated over an angle $\delta$, which can be measured using a rotating analyzer.

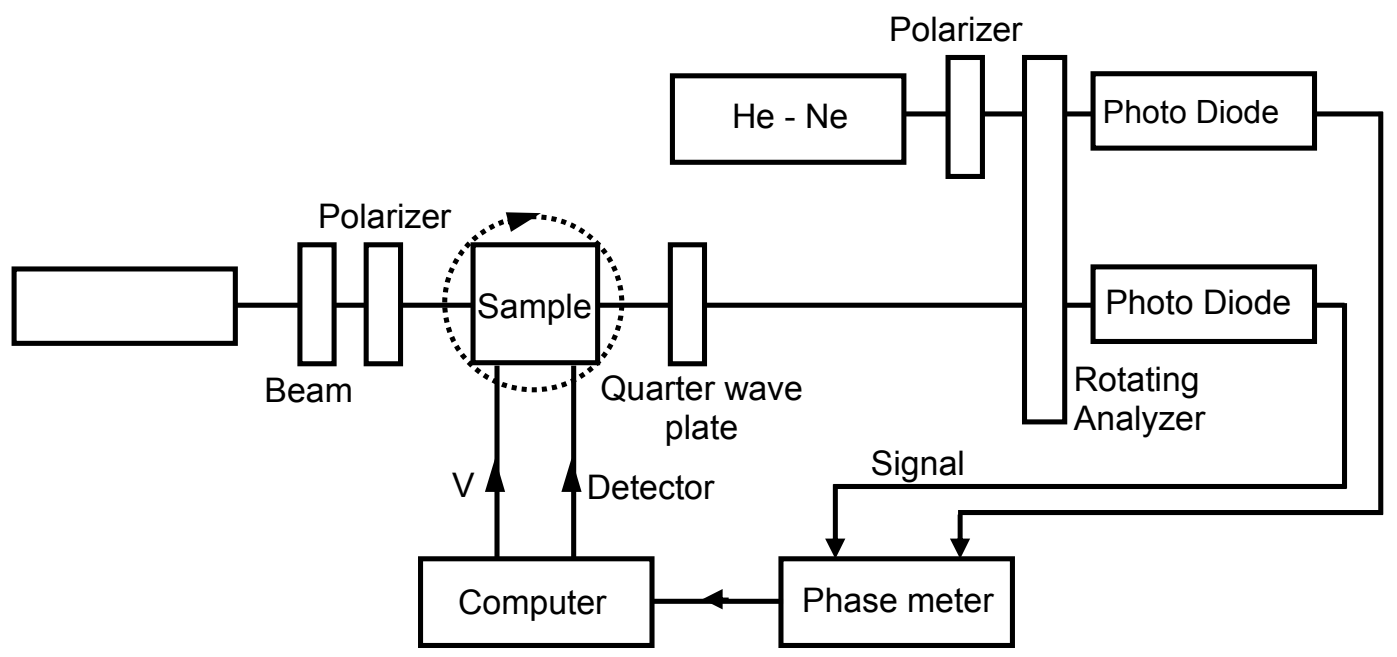

Figure 2.2: Setup used for the determination of pretilt angle, which uses the crystal rotation method.

\subsection{Results and discussion}

\subsection{1 $\mu$-Rubbing of unrubbed and pre-rubbed polyimide: Surface characterization}

In an initial set of experiments, the alignment of liquid crystals on polyimide substrates after rubbing with a metallic sphere $(\mathrm{d}=1 \mathrm{~mm})$ was investigated. Figure 2.3 shows the scanning electron and atomic force microscopy (AFM) image of the metallic sphere used for the $\mu$-rubbing process. The metallic sphere has an average surface roughness of $8 \pm 2$ 
$\mathrm{nm}$, measured using AFM. The sphere is connected to a cantilever, which accurately controls the force $(1-100 \mathrm{~N})$ exerted on the polyimide coated substrate. The substrate is mounted on a translational stage that moves with a pre-determined velocity.

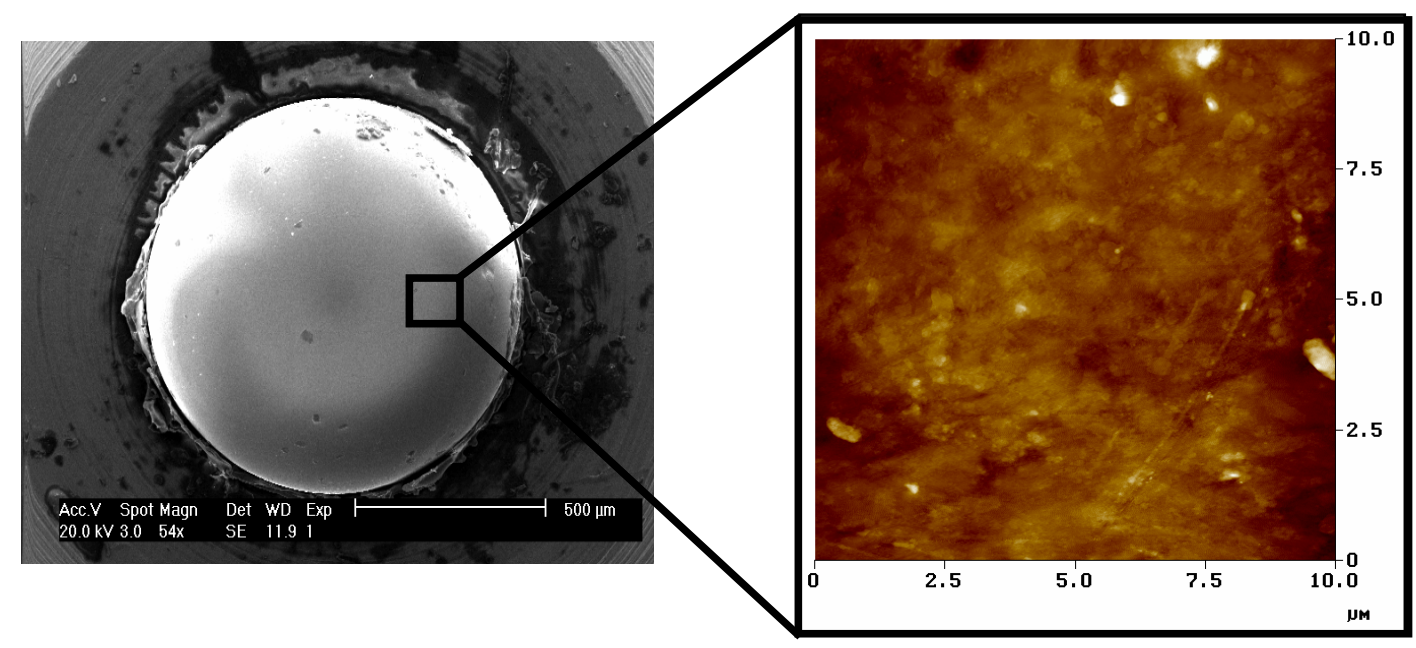

(a)

(b)

Figure 2.3: Scanning electron (a) and atomic force microscopy (b) images of the metallic sphere used in the $\mu$-rubbing process.

The $\mu$-rubbing of a spin coated, baked polyimide was initially investigated. In Fig. 2.4, AFM measurements are shown of polyimide surfaces which were $\mu$-rubbed at two different loads, $4 \mathrm{~g}$ and $20 \mathrm{~g}$. The figures illustrate that the spin coated polyimide layers are extremely smooth outside the recorded patterns. Within the recorded lines, irregular spaced grooves are observed (see the height profile in Fig. 2.4 top), which are oriented in the recording direction. Some debris is also visible especially at high loads (Fig. 2.4b). 

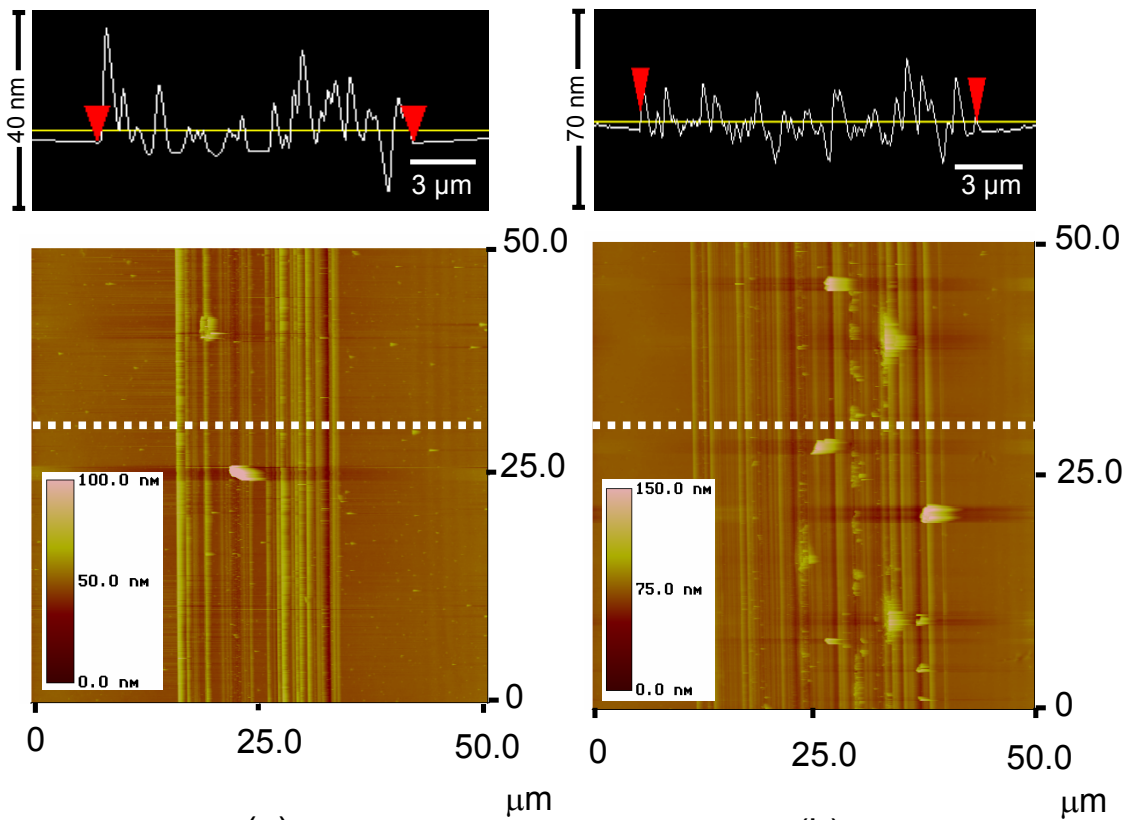

(a)

(b)

Figure 2.4: Atomic force microscopy images of $\mu$-rubbed polyimide with a load of $4 \mathrm{~g}(\mathrm{a}), 20 \mathrm{~g}$ (b) and their height profiles along the dotted line (top).

Next, a pre-rubbing procedure was performed with a velvet cloth (conventional rubbing), which induces a homogeneous planar alignment in the liquid crystal layer. The $\mu$-rubbing of these polyimide layers was performed in a direction perpendicular to the pre-rubbing direction. Figure 2.5 shows AFM images at a scanning angle of $45^{\circ}$ of the pre-rubbed, $\mu$-rubbed substrates at two different loads. Outside the recorded patterns, the subtle evidence of grooves from the first rubbing procedure can be observed. Upon $\mu$ rubbing, well defined line patterns are generated with irregular grooves parallel to the $\mu$ rubbing direction. The actual roughness originating from the $\mu$-rubbing procedure exceeds the roughness from the conventional rubbing even at low loads $(<2 \mathrm{~g})$, which is the first indication that the initial buffing is erased by the $\mu$-rubbing.

The depth of the $\mu$-rubbed grooves was determined. From the AFM experiments it was found that the depth is $10-20 \mathrm{~nm}$, almost one order of magnitude less than the thickness of the spin-coated polyimide film $(\sim 100 \mathrm{~nm})$. Scanning electron microscopy 
images of pre-rubbed, $\mu$-rubbed polyimide at two different loads are shown in Fig. 2.6. At higher loads $(20 \mathrm{~g})$ the formation of debris is again clear in the picture.
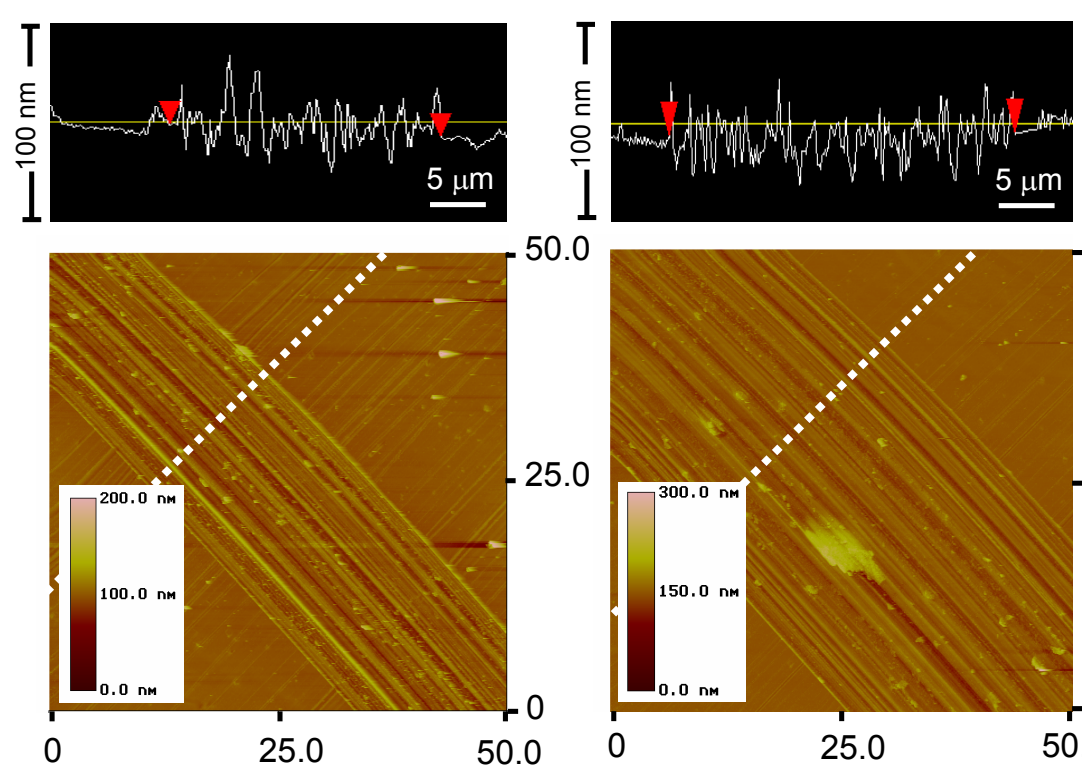

50.0

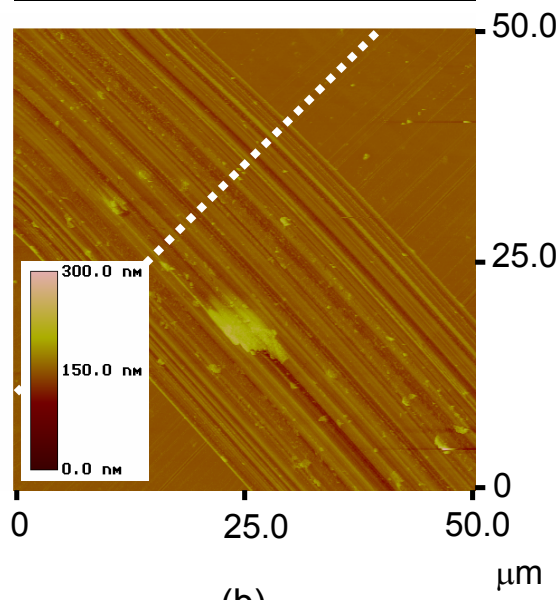

(a)

(b)

Figure 2.5: Atomic force microscopy images of pre-rubbed $\mu$-rubbed polyimide with a load of (a) $4 \mathrm{~g}$ and (b) $20 \mathrm{~g}$ and its height profile (top) along the dotted line.

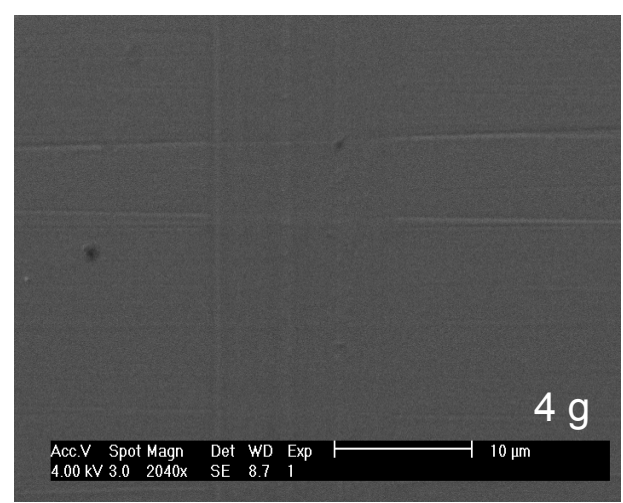

(a)

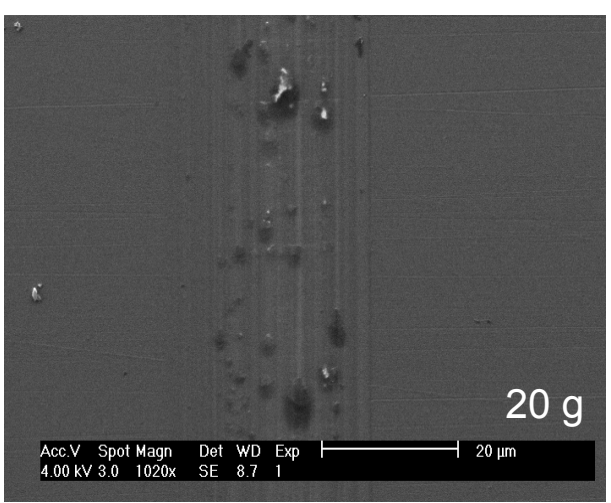

(b)

Figure 2.6: Scanning electron microscopy images of the pre-rubbed, $\mu$-rubbed polyimide, at $4 \mathrm{~g}$ (a) and $20 \mathrm{~g}(\mathrm{~b})$, the amount of debris increases with applied load, which is visible at $20 \mathrm{~g}$ load. 
In Fig.2.7 the line width of the recorded patterns is plotted as a function of the applied load. Experimental data is used originating from line width measurements based on AFM (on bare substrates) and optical microscopy (on cells filled with liquid crystal). It is shown that a relationship exists between the applied load and the line width of the pattern. Moreover, this graph illustrates the flexibility in creating different line widths using a metallic sphere of $1 \mathrm{~mm}$ diameter.

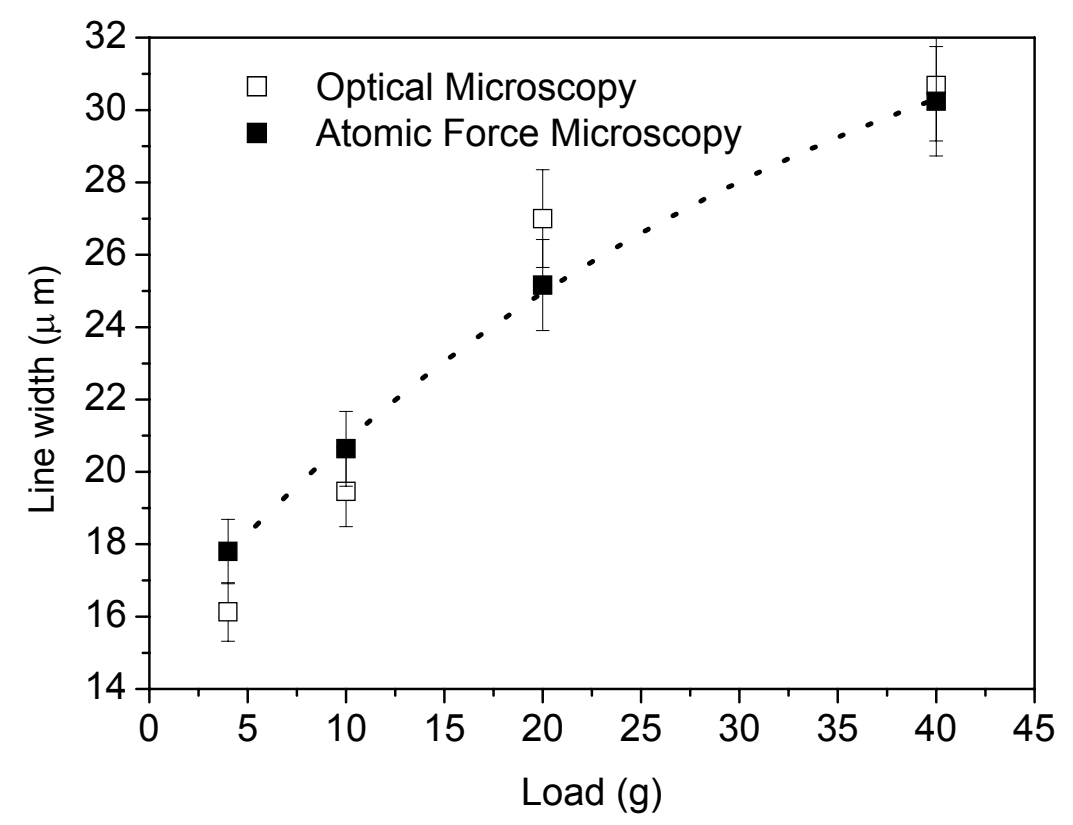

Figure 2.7: Variation of line width with applied load, measured with atomic force microscopy and with polarized optical microscopy (E7 filled cell).

\subsubsection{Alignment of LCs on $\mu$-rubbed surface}

The $\mu$-rubbing was performed on polyimide before and after the imidization step to study the effect of curing reaction on LC alignment. Figure 2.8 shows different methods used to construct an LC cell. From the pre-baking stage three different procedures were followed as shown below. 


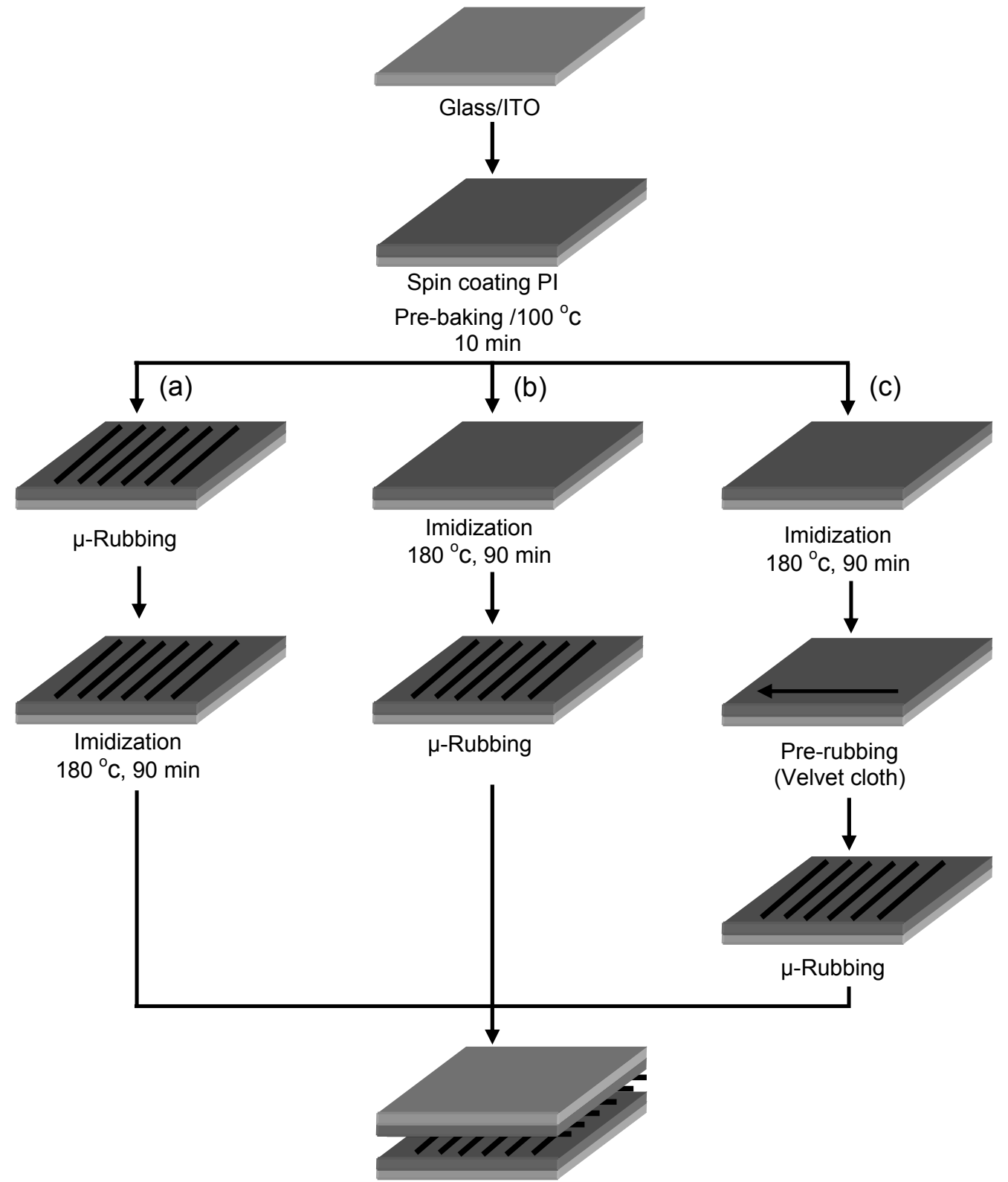

Cell construction

Figure 2.8: Depicts the different steps followed for the cell preparation (a) $\mu$-rubbing before imidization, (b) $\mu$-rubbing after imidization and (c) $\mu$-rubbing on pre-rubbed polyimide.

Liquid crystal cells were constructed with two unrubbed, $\mu$-rubbed polyimide substrates (Fig. 2.8 paths (a) and (b)). The $\mu$-rubbing was performed before (a) and after 
(b) the imidization process. The rubbing directions of the top and bottom substrate are orthogonal to each other. In Fig 2.9 ( $\mu$-rubbing before imidization) and Fig. 2.10 ( $\mu$ rubbing after imidization) optical micrographs of the liquid crystal cells are shown.

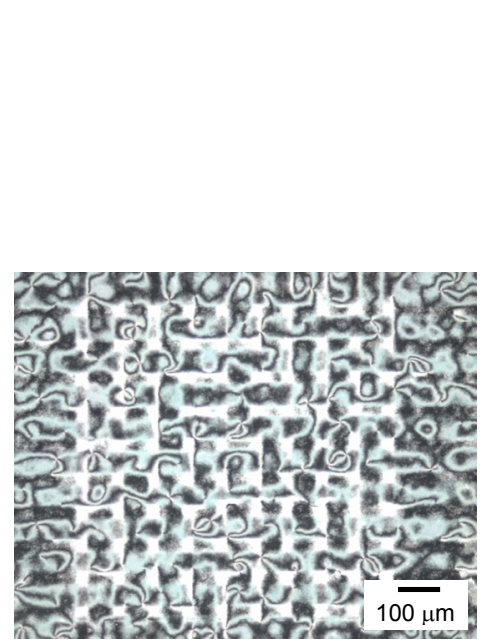

(a)

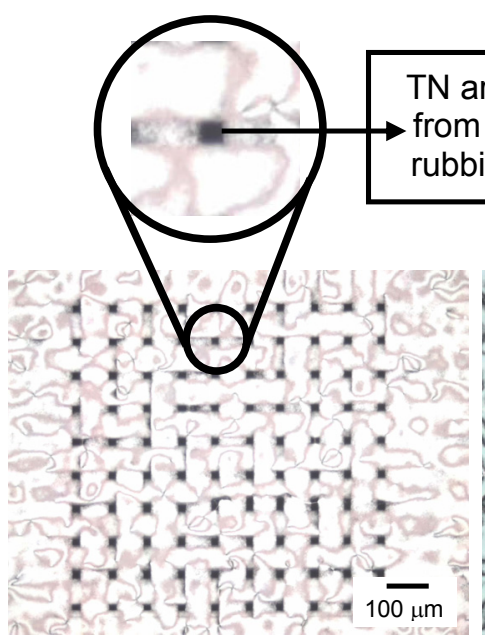

(b)

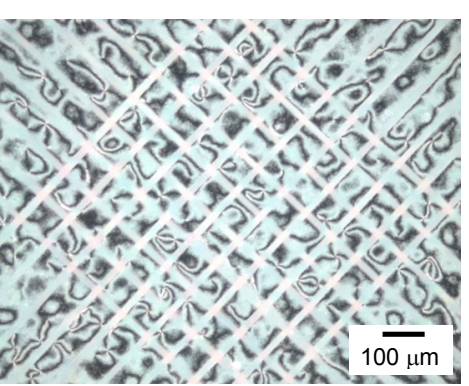

(c)

Figure 2.9: $\mu$-rubbing before imidization (path (a) in Fig. 2.8): Optical micrographs of E7 filled cells with unrubbed, $\mu$-rubbed polyimide on both sides, crossed pattern (load: $20 \mathrm{~g}$ ) between crossed polarizers (a) between parallel polarizers (b) and cell at $45^{\circ}$ between crossed polarizers (c). Magnification 10X.

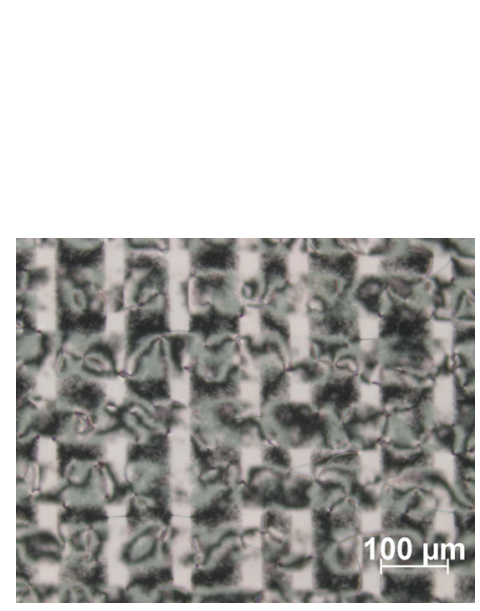

(a)

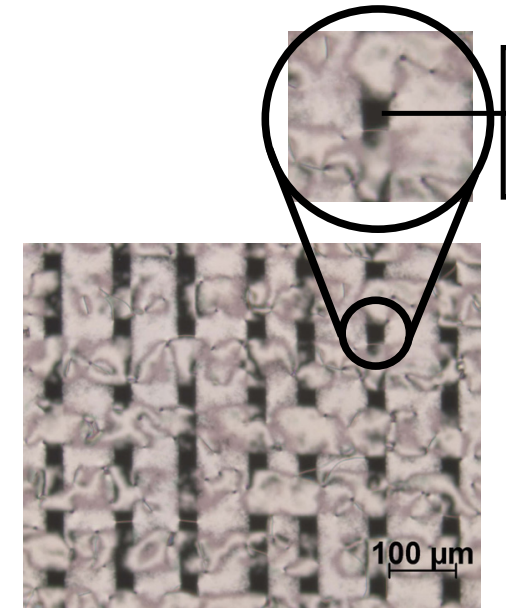

(b)

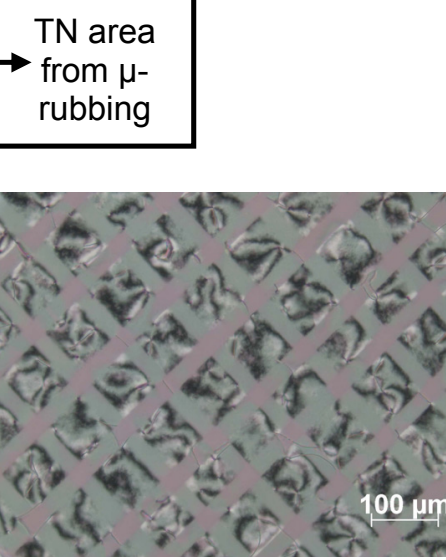

(c)

Figure 2.10: $\mu$-rubbing after imidization (path (b) in Fig.2.8): Optical micrographs of E7 filled cells with unrubbed, $\mu$-rubbed polyimide on both sides, crossed pattern (load: 20 g) between (a) crossed polarizers, (b) between parallel polarizers and (c) cell at $45^{\circ}$ between crossed polarizers. Magnification 20X. 
In both cases homogenous alignment is expected in the $\mu$-rubbed area. This is confirmed by observation of twisted nematic areas at points where the two $\mu$-rubbed lines intersect. Outside the $\mu$-rubbed area, typical Schlieren texture is observed. Alignment of the liquid crystals is observed in both cases with $\mu$-rubbing before and after imidization.

Liquid crystal cells were also made with pre-rubbed, $\mu$-rubbed polyimide on one side (path (c) in Fig. 2.8) and pre-rubbed polyimide on the other side. Figure 2.11 shows the optical micrograph of the cell viewed between polarizers. Here, homogenous line patterns are observed in the $\mu$-rubbed areas, which indicate that the pre-rubbing (buffing) history is erased by the $\mu$-rubbing process.

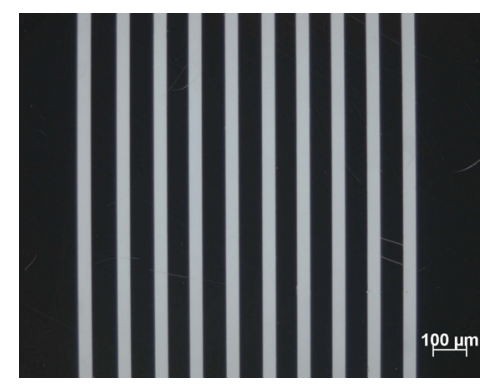

(a)

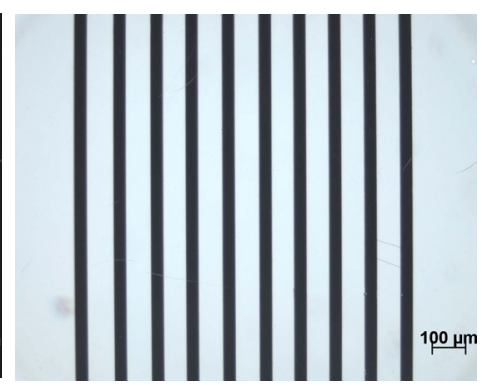

(b)

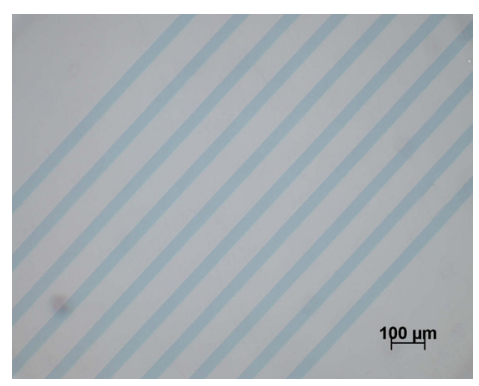

(c)

Figure 2.11: Optical micrographs of E7 filled cells with pre-rubbed $\mu$-rubbed polyimide on one side and pre-rubbed polyimide on the other (path (c) in Fig. 2.8; rubbing load: 20 g) between parallel polarizers (a), between crossed polarizers (b) and cell at $45^{\circ}$ between crossed polarizers (c).

Cells were also constructed with two homogeneously pre-rubbed polyimide layers containing several $\mu$-rubbed lines as shown in Fig. 2.12. In these cells, the rubbing directions are mutually orthogonal which creates several regions in the same cell: (i) twisted nematic regions originating from the pre-rubbing procedure with a velvet cloth; (ii) planar regions originating from the combination of a homogeneous pre-rubbed layer with a $\mu$-rubbed line; and (iii) twisted nematic regions originating from the $\mu$-rubbing procedure. The optical micrographs illustrate that well defined regions with different liquid crystal configurations are obtained. 


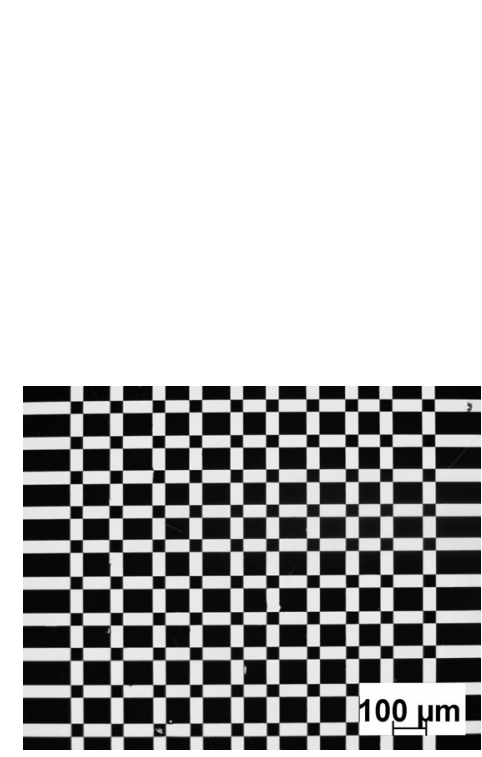

(a)

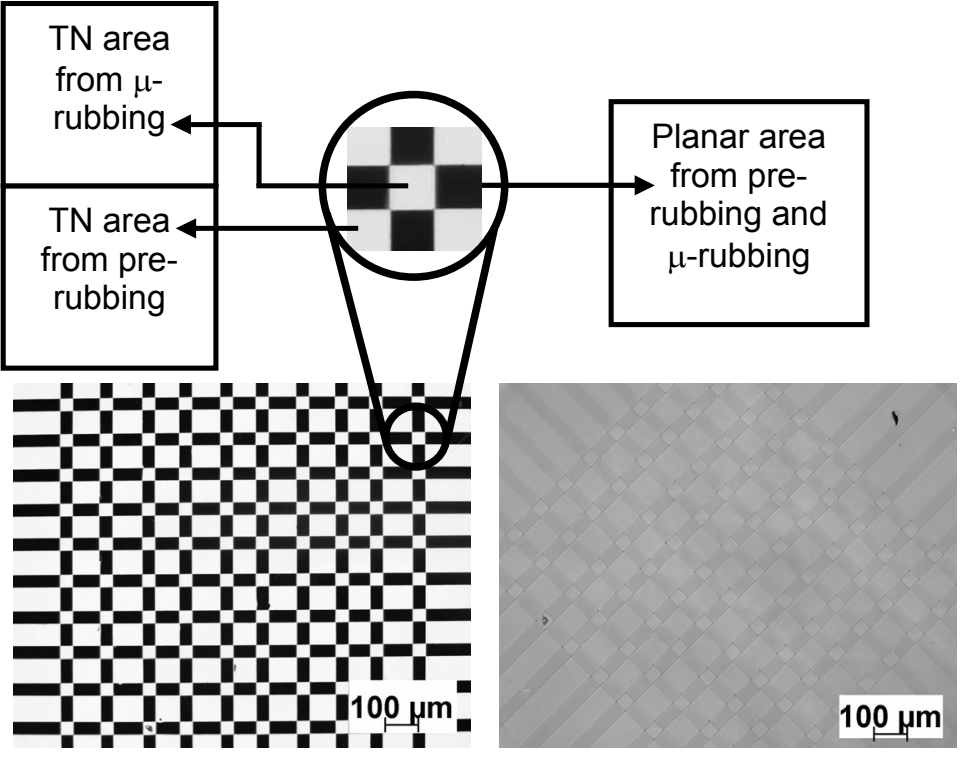

(b)

(c)

Figure 2.12: Optical micrographs of E7 filled cell with pre-rubbed polyimide substrate having $\mu$ rubbed, crossed pattern on both sides are shown between (a) parallel polarizers, (b) between crossed polarizers and (c) the cell at $45^{\circ}$ between crossed polarizers.

\subsubsection{Pretilt angle measurements: Conoscopic technique}

The surface pretilt angle (unidirectional tilt of the LC molecules from the rubbing direction is called the pretilt angle) of the pre-rubbed and the $\mu$-rubbed polyimide was measured using a conoscopic technique with an Autronic DMS 703 instrument. ${ }^{22}$ Antiparallel cells were made with several recorded parallel lines with a cell spacing of $18 \mu \mathrm{m}$ and placed between crossed polarizers with the optic axis parallel to the bottom polarizer direction. Figure 2.13 shows the conoscopic images of pre-rubbed and $\mu$-rubbed polyimide. For perfectly planar alignment of the liquid crystal at the surface with zero pretilt, the conoscopic pattern is characterized by a family of hyperboles that are symmetrically arranged in four quadrants. The shift of the pattern about the origin is a rough measure of the surface pretilt angle. The pretilt was measured by carefully analyzing the position of the hyperbolic lines about the origin. ${ }^{23}$ It was found that the 
polyimide from the conventional rubbing method induces a pretilt angle of $2.65^{\circ} \pm 0.5$, consistent with specifications from the manufacturer.

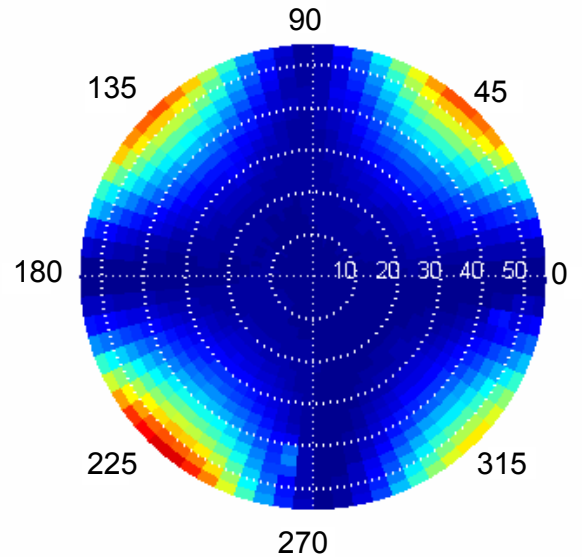

(a)
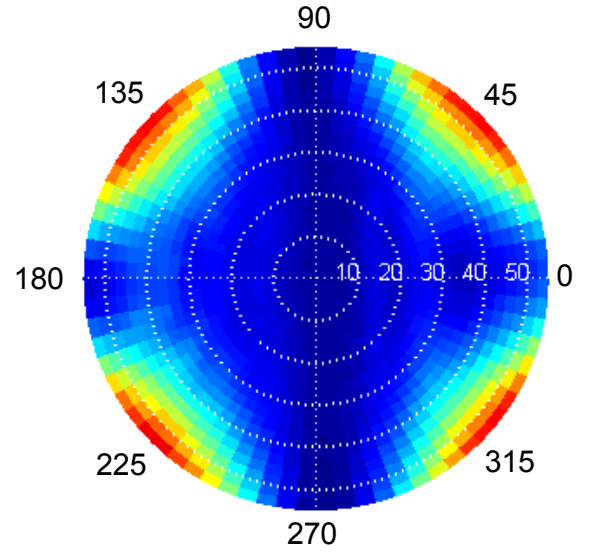

(b)

Figure 2.13: Conoscopic images of the anti-parallel cells with 18 um cell gap (a) polyimide by conventional rubbing (b) from $\mu$-rubbing.

In the case of the $\mu$-rubbed polyimide, the conoscopic image is nearly symmetrical, which indicates that the average pretilt is small. A subtle asymmetry of the conoscopic images can be observed in Fig. 2.13a. For the exact measurement of the pretilt angle in the $\mu$-rubbed area an Autronic TBA 107 instrument was used. This instrument uses a crystal rotation method and found a pretilt angle of $0.7^{\circ}$.

\subsubsection{Alignment of LCs in an applied field}

When an electric field is applied to a TN cell, the transmission between crossed polarizers remains identical for applied voltages between zero and threshold voltage $\left(V_{T}\right)$. As the voltage increases beyond $V_{T}$ the liquid crystal molecules are tilted toward the direction of the electric field, which decreases the phase retardation and leads to an increase in transmission. At high voltages, the LCs adopts a homeotropic alignment and the light is almost completely absorbed by the analyzer resulting in a black state. In Fig. 2.14 ( $\mu$-rubbing before imidization) and Fig. 2.15 ( $\mu$-rubbing after imidization) the response of the electro-optical cells at different voltages are shown. In both cases the twisted nematic areas originating from the intersection of the $\mu$-rubbed lines from the top 
and the bottom substrates switch to a black state. There is no noticeable difference in switching behavior of the polyimide before and after imidization i.e. both cells switch at approximately the same voltage.
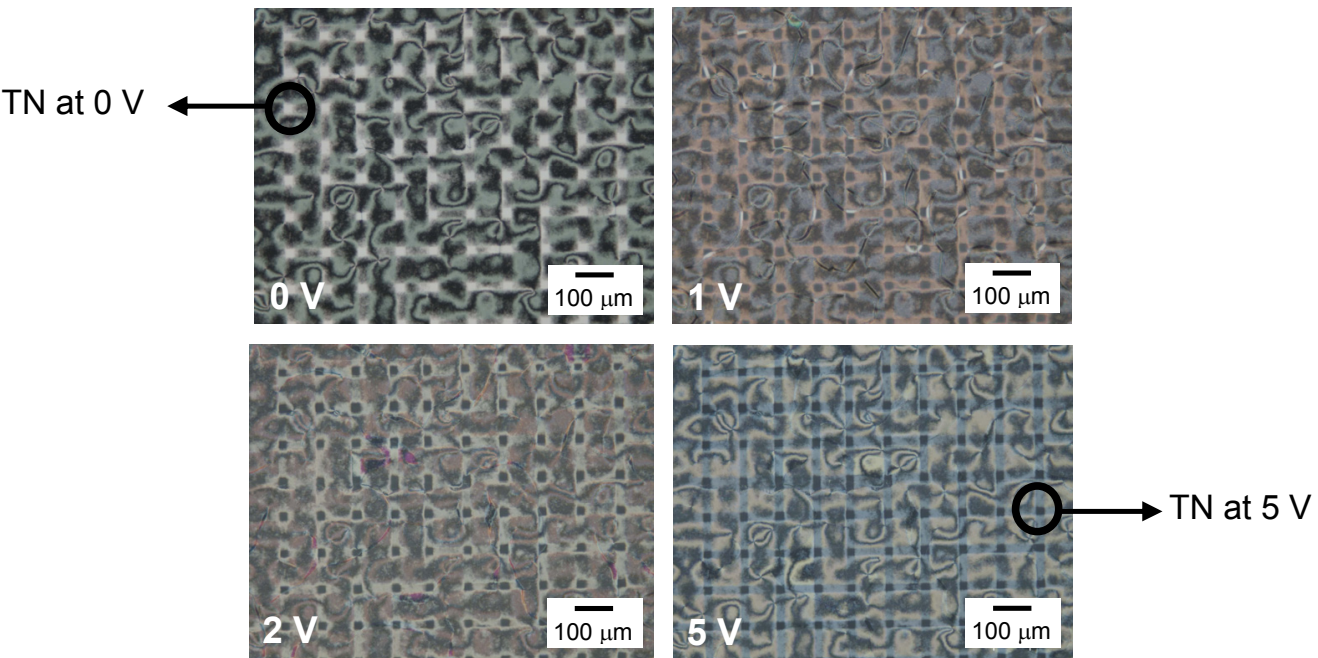

Figure 2.14: $\mu$-rubbing before imidization: Optical micrographs of E7 filled cells with unrubbed, $\mu$-rubbed polyimide on both sides, crossed pattern (load: $20 \mathrm{~g}$ ) between crossed polarizers under different electric fields. Magnification 10X.
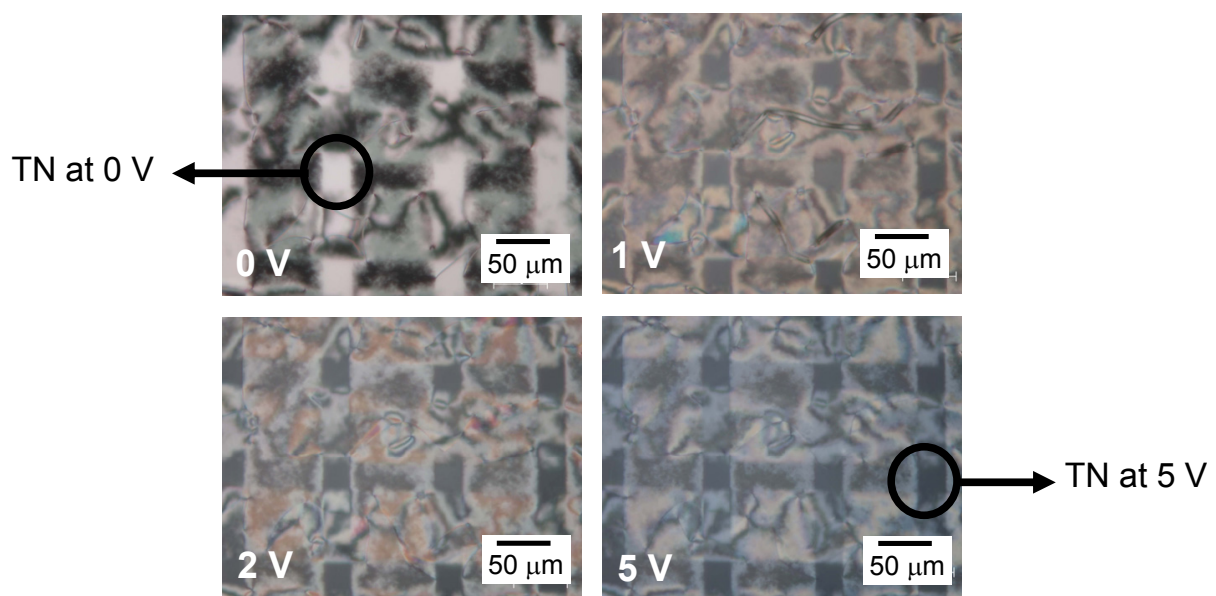

Figure 2.15: $\mu$-rubbing after imidization: Optical micrographs of E7 filled cells with unrubbed, $\mu$-rubbed polyimide on both sides, crossed pattern (load: $20 \mathrm{~g}$ ) between crossed polarizers under different electric fields. Magnification 20X.

In Fig. 2.16, optical micrographs are shown of an LC cell prepared according to path (c) in Fig. 2.8 (pre-rubbed and $\mu$-rubbed) in an applied electrical field. The twisted 
nematic regions originating from the buffing and $\mu$-rubbing have similar threshold voltages and they switch at almost same voltage. In the planar regions, the threshold voltage is slightly lower than in the TN regions as predicted by a Fredericks transition model. ${ }^{24}$ Also, bright lines at the interfaces between the various alignment regions are clearly observable, especially at intermediate and high voltages. These lines often are referred to as disclination lines. The alignment in these areas between the twisted and untwisted regions give rise to bright lines that will be discussed in the coming sections.

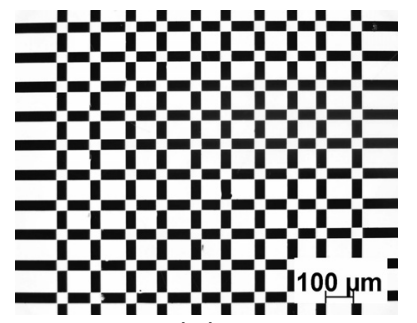

(a)

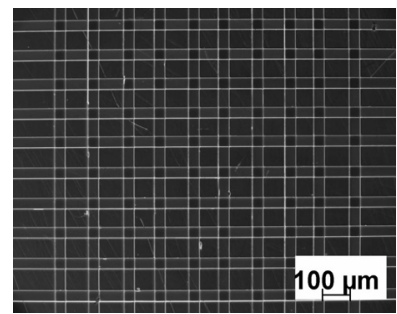

(d)

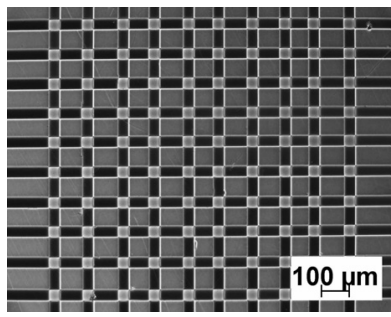

(b)

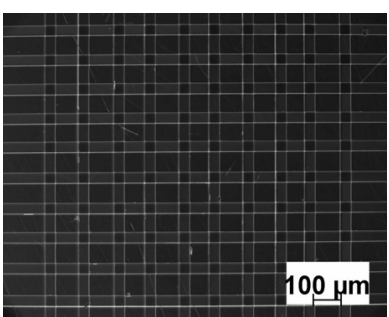

(e)

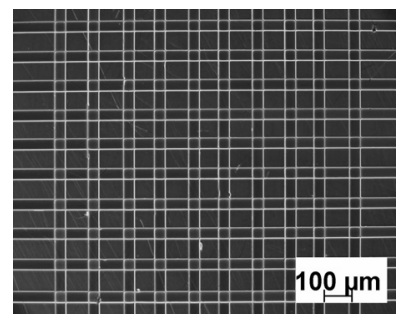

(c)

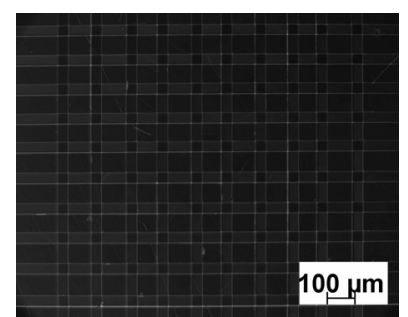

(f)

Figure 2.16: Optical micrographs of LC cell with pre-rubbed polyimide having $\mu$-rubbed patterns viewed between crossed polarizers, response under electric field. (a) $V=0$, (b) $V=1.0$, (c) $V=2.0$, (d) $V=4.0$, (e) $V=6.0$, (f) $V=10.0$.

In Fig. 2.17 the intensity profile of the twisted nematic areas is shown as a function of applied voltage ( 0 to $5 \mathrm{~V}$ ) in the normally white state (crossed polarizers). Optical micrographs were recorded and a line scan was performed (see figure inset) on these micrographs at different voltages. Subsequently, the light intensity is plotted versus the position within the cell. Starting from a bright state $(0 \mathrm{~V})$ the intensity of the twisted nematic area decreases with applied field. At a higher voltage (approximately $2 \mathrm{~V}$ ), disclination lines, manifesting themselves as bright lines at the edges of the twisted nematic regions, are formed due to a difference in the liquid crystal orientation direction. 
The light intensity of the planar area is shown in Fig. 2.18 as a function of voltage. The planar area is black between crossed polarizers and remains black with applied field. At high voltages, light leakage (disclination lines) is observed at the interface between planar and twisted nematic regions.

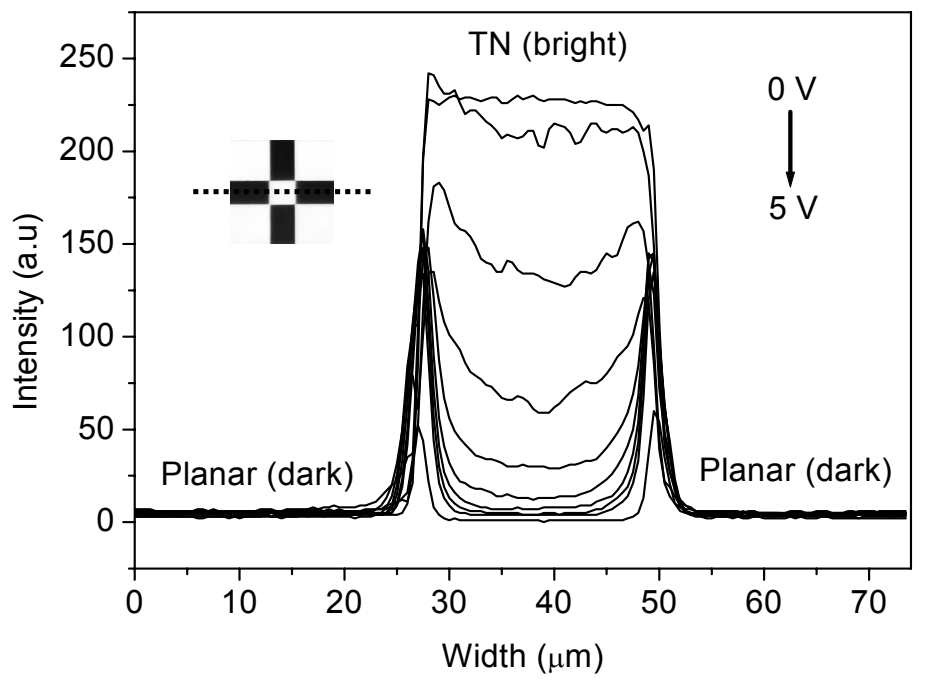

Figure 2.17: The light intensity profile of the twisted nematic area by the $\mu$-rubbing process at different electric fields, obtained by line scan of the optical micrograph (inset, dotted line shows the line scanning area) from $0 \mathrm{~V}$ to $5 \mathrm{~V}$ with an interval of $0.5 \mathrm{~V}$.

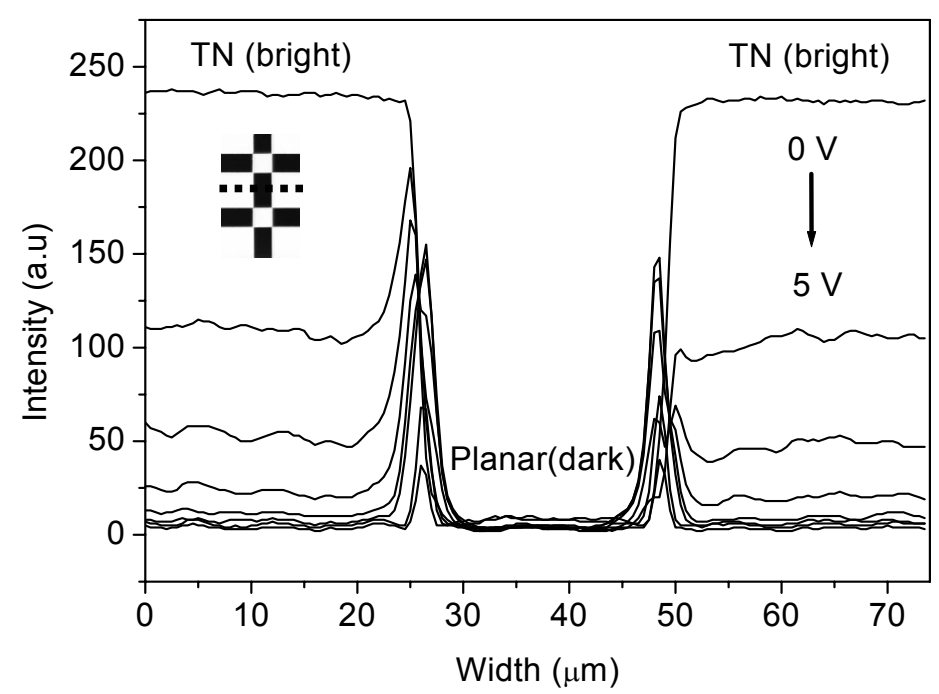

Figure 2.18: The intensity profile of the planar area by the $\mu$-rubbing process at different electric fields, obtained by the line scanning of the optical micrograph from (inset, dotted line shows the line scanning area) $0 \mathrm{~V}$ to $5 \mathrm{~V}$ with an interval of $0.5 \mathrm{~V}$. 


\subsubsection{Optical simulations}

\subsubsection{Director orientations and transmittance}

The liquid crystal director orientation in the twisted nematic and planar area was simulated using the 2dimMOS program (see theoretical Section 2.2) at 0 and $4 \mathrm{~V}$. Figures 2.19 and 2.20 show the vertical cross section of the cell and the director orientations of the twisted nematic area at 0 and $4 \mathrm{~V}$ respectively. The gray rods represent the LC directors, where the light end indicates a forward direction and the dark ends indicate a backward direction with respect to the plane of drawing. The pretilt angle $\left(\theta_{p}\right)$ and liquid crystal director directions (azimuthal angle, $\varphi$ ) used in the calculations are indicated in Fig. 2.19. From the simulation the orientation of directors and the formation of disclination lines are investigated. The values for the pretilt and azimuthal angle were derived from the experimental measurements in paragraph 2.4.3. The calculations were done with crossed polarizers, parallel and perpendicular to plane of drawing. Table 2.1 shows the parameters of TN cell used in the optical simulations.

Table 2.1: Parameters of TN cell used in the optical simulations. (The material parameters of LC E7 are used.)

\begin{tabular}{cll}
\hline \hline & & \\
Dielectric anisotropy & $\varepsilon_{/ /}$ & 19.0 \\
$\left(20^{\circ} \mathrm{C}, 1 \mathrm{kHz}\right)$ & $\varepsilon_{\perp}$ & 5.2 \\
& $\Delta \varepsilon$ & +13.8 \\
Elastic constants $\left(20^{\circ} \mathrm{C}\right)$ & $k_{11}$ & $11.1 \mathrm{pN}$ \\
& $k_{33}$ & $17.1 \mathrm{pN}$ \\
& $k_{33} / k_{11}$ & 1.54 \\
Optical anisotropy & $\Delta n$ & 0.2253 \\
$\left(20^{\circ} \mathrm{C}, 589 \mathrm{~nm}\right)$ & $n_{e}$ & 1.7464 \\
Cell gap $(\mu \mathrm{m})$ & $n_{o}$ & 1.5211 \\
& $d$ & 5.0 \\
Pretilt angles & & \\
& $\theta_{p}$ & $2.6^{\circ}, 0.7^{\circ}$ \\
\hline
\end{tabular}


In Fig. 2.19 and Fig. 2.20, the top part has uniform pretilt angle from the $\mu$ rubbing throughout the section, but in the bottom part the cell is divided into three parts; left, middle and right. The left and right parts have the same LC orientation which originates from the pre-rubbing. In the middle portion, the $\mathrm{LC}$ orientation is perpendicular to the pre-rubbing direction due to $\mu$-rubbing and the LC directors are away from the viewer, which creates a TN area as shown in the figure. In an applied field $(4 \mathrm{~V})$ the LC director changes to the homeotropic state, (note that close to the surface the homeotropic alignment is not perfect). The left and right regions are black since the directors are parallel to one of the polarizers. In the central region the rest retardation of the LC close to the top and bottom compensate each other. At the boundaries between the pre-rubbing and $\mu$-rubbing regions this compensation is not perfect and hence light leakage occurs leads to the formation of disclination lines. 


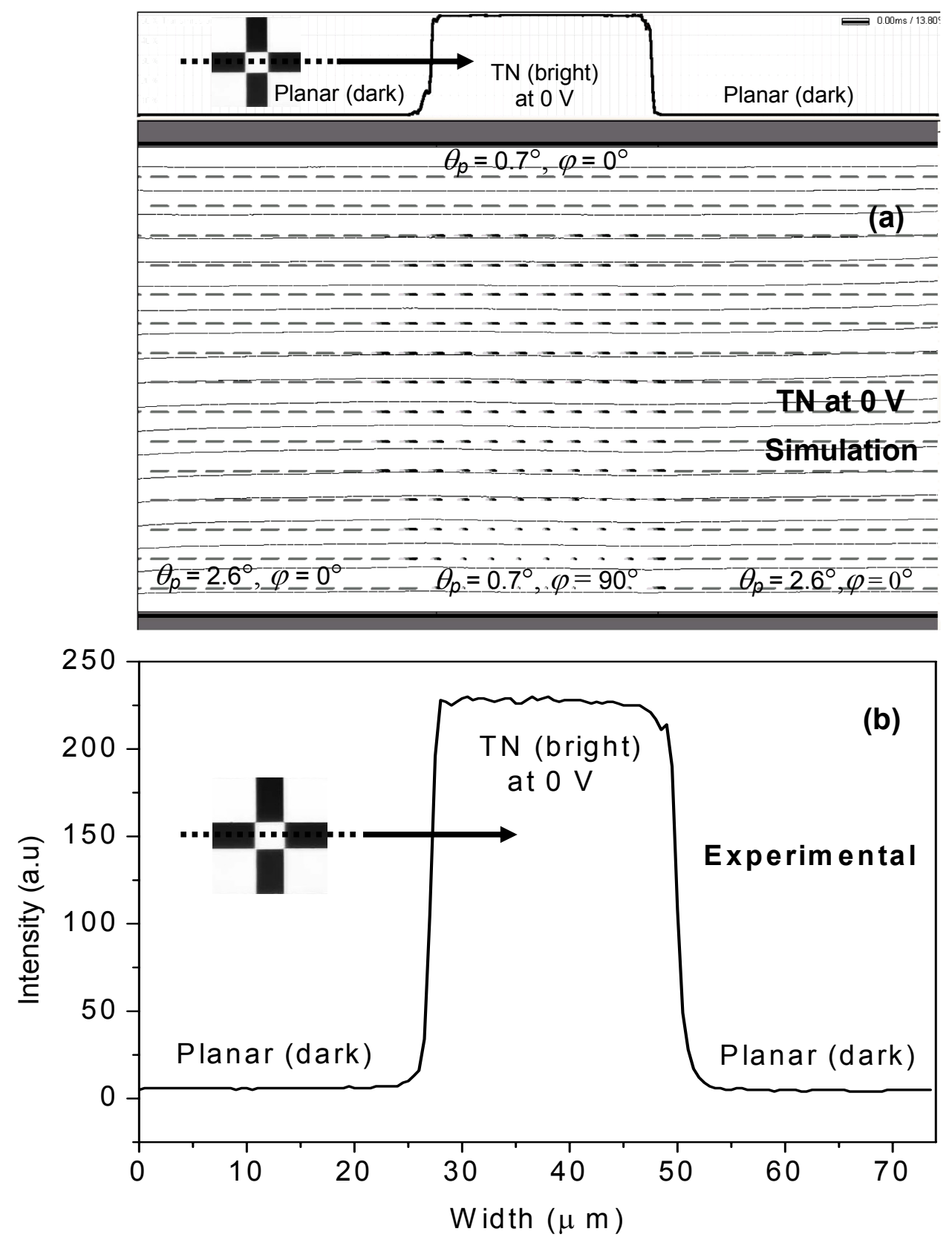

Figure 2.19: Comparison of transmittance of $T N$ area at $0 \mathrm{~V}$ from optical simulation (a) and experimental results (b), obtained by the line scanning of the optical micrograph shown in the inset. The director orientations of the TN area at $0 \mathrm{~V}$ from the 2dimMOS calculations is also shown in (a). 


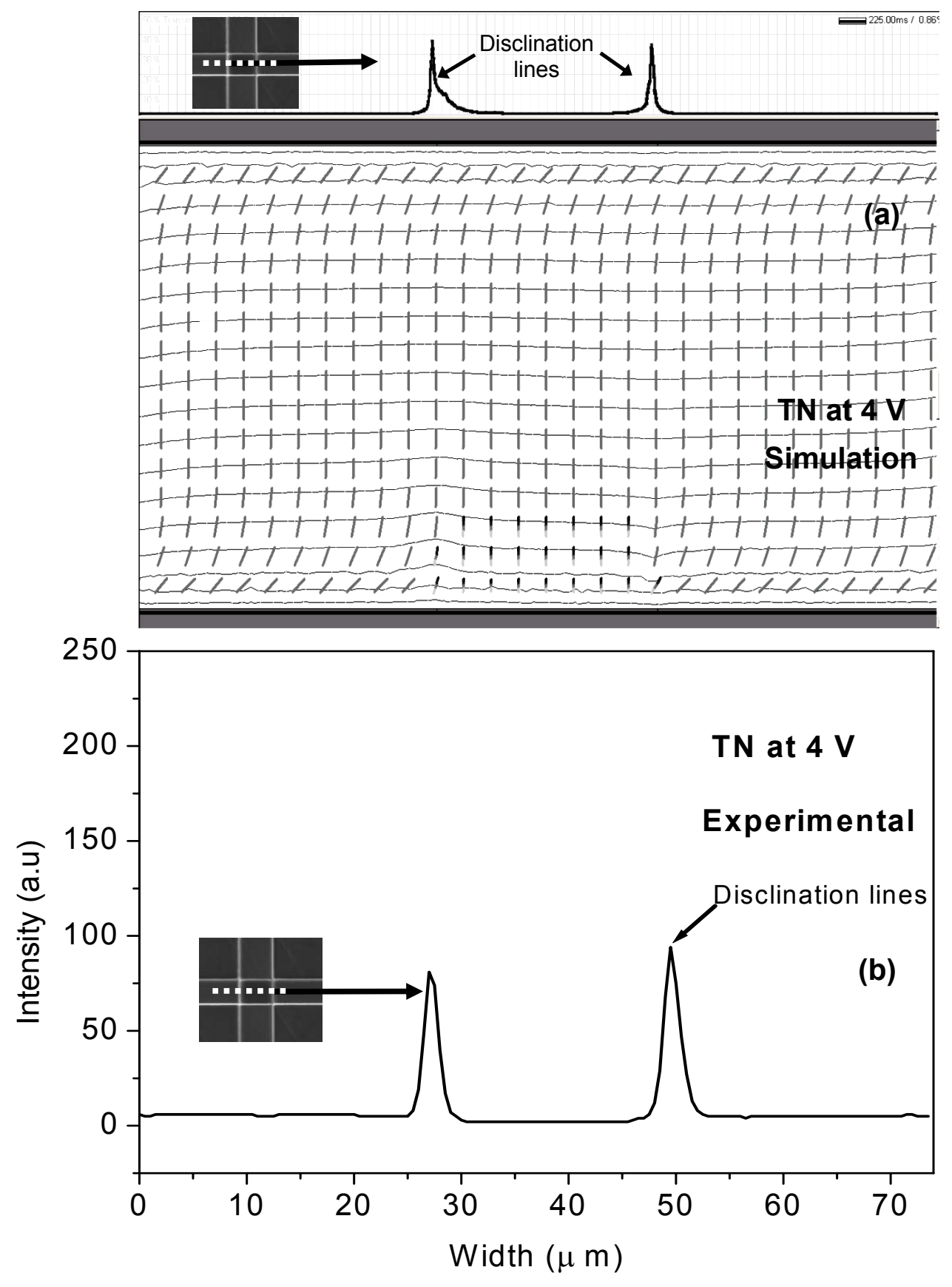

Figure 2.20: Transmittance of TN area at $4 \mathrm{~V}$ from optical simulation (a) and experimental results (b), obtained by the line scanning of the optical micrograph shown in the inset. The director orientations from the 2 dimMOS calculation of the $T N$ area at $4 \mathrm{~V}$ are also shown. 

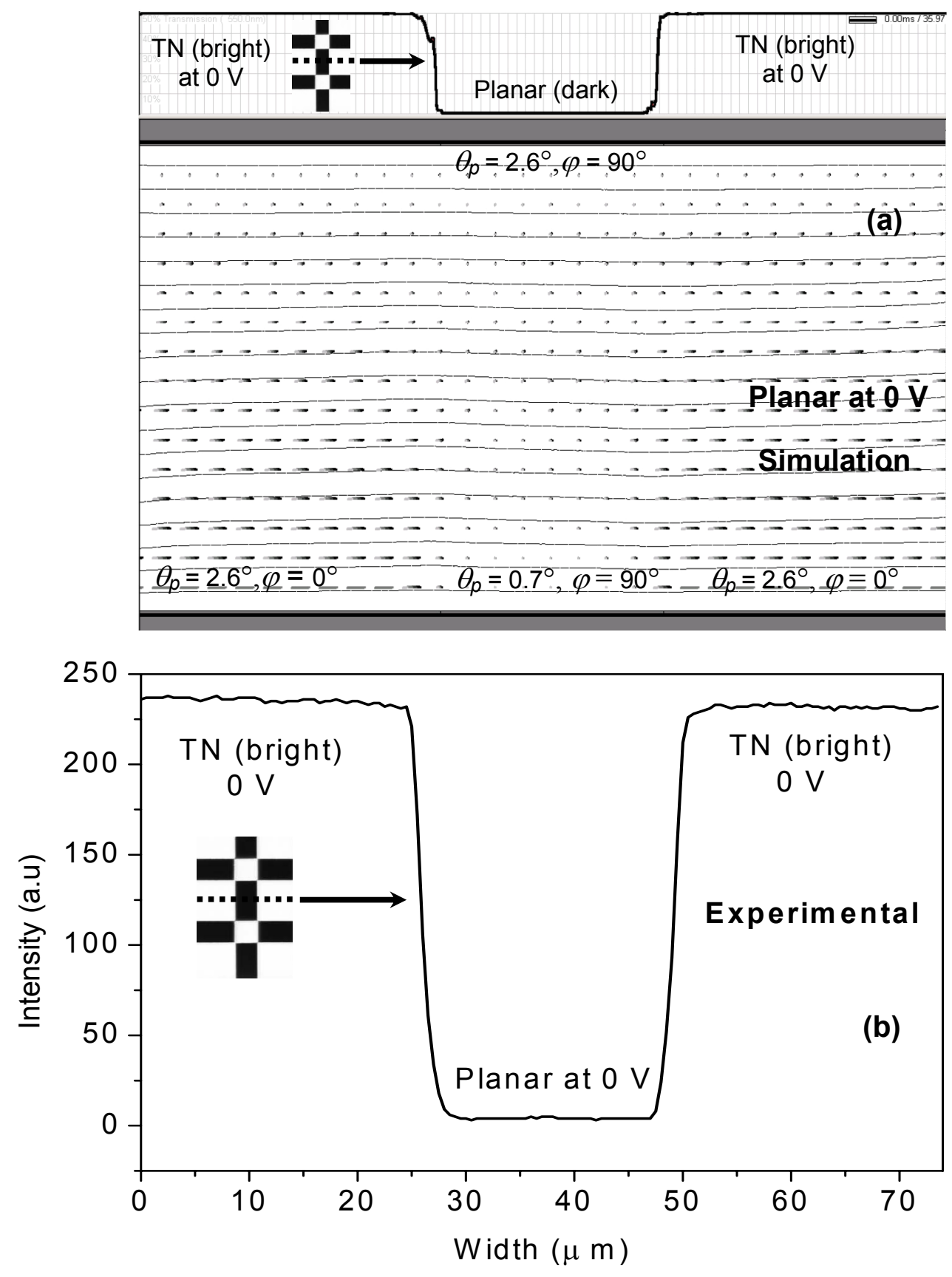

Figure 2.21: Transmittance of planar area at $0 \mathrm{~V}$ from optical simulation (a) and experimental results (b), obtained from a line scan of the optical micrograph shown in the inset. The director orientations from the 2 dimMOS calculation of the planar area at $0 \mathrm{~V}$ are also shown. 


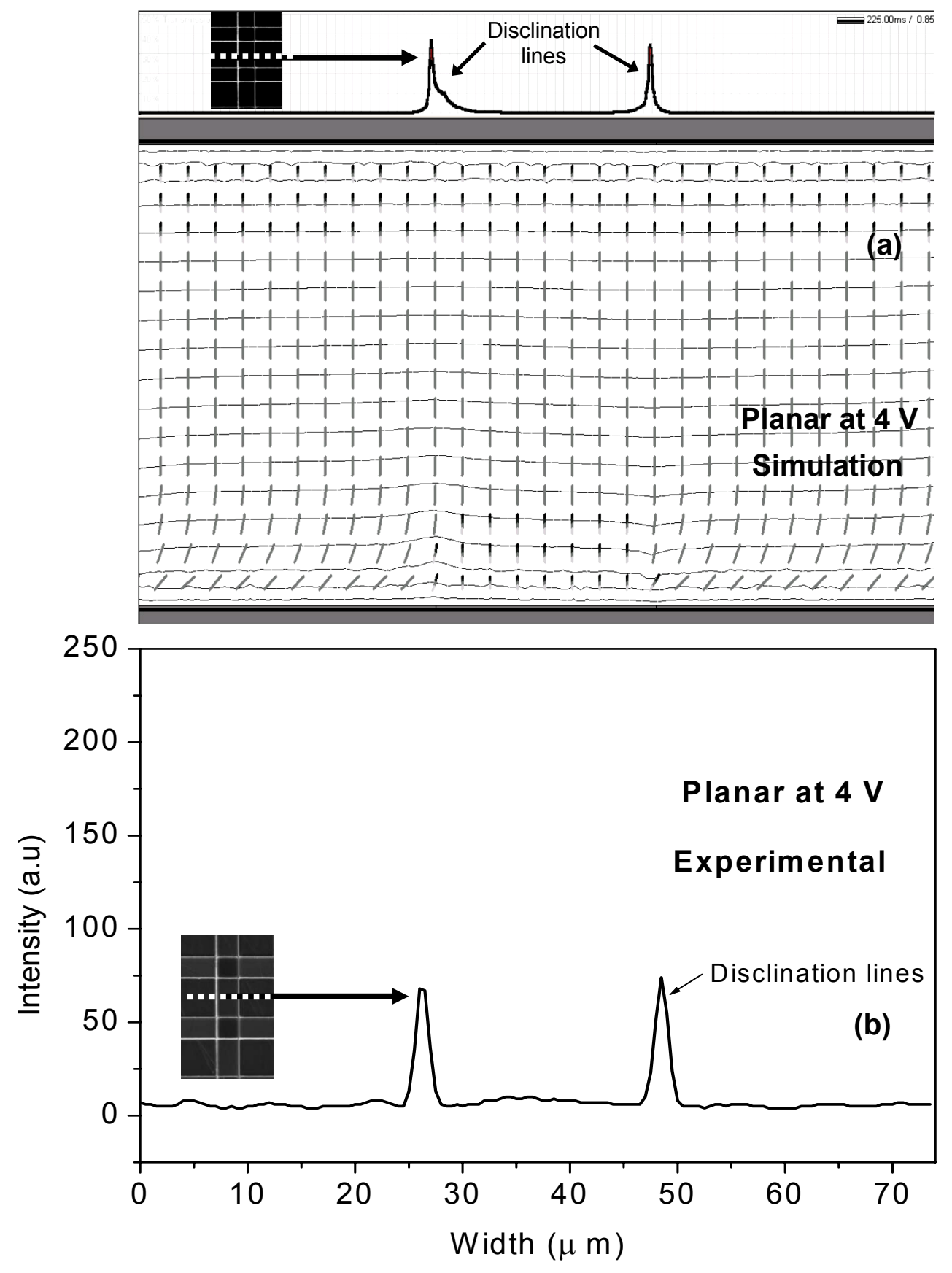

Figure 2.22: Comparison of transmittance of planar area at $4 \mathrm{~V}$ from optical simulation (a) and experimental results (b), obtained by the line scanning of the optical micrograph shown in the inset. The director orientations from the 2 dimMOS calculation of the planar area at $4 \mathrm{~V}$ are also shown. 
Figure 2.21 and Fig. 2.22 shows a comparison of calculated and experimental transmittance, director orientation in the planar area having $\mathrm{TN}$ on both sides at 0 and 4 V. Here the top substrate is pre-rubbed and bottom substrate is $\mu$-rubbed. The pretilt and the LC director directions are indicated in Fig. 2.21. The vertical cross section of the cell possesses a uniform pretilt at the top substrate. As mentioned above the bottom portion consists of left, middle and right part, in which the middle part is $\mu$-rubbed perpendicular to the pre-rubbing direction. The $\mu$-rubbed portion of the bottom substrate and the prerubbed top substrate gives rise to a planar alignment with a dark region as shown in the figure at $0 \mathrm{~V}$. In an applied field the directors align vertically (homeotropic state). Again in the regions between the pre-rubbed and $\mu$-rubbed areas there is some uncompensated rest retardation giving rise to disclination lines in the optical micrograph and in the 2dimMOS calculations.

\subsubsection{Electro-optical characterization}

The $\mu$-rubbing process with a metallic ring (see experimental section) was used to record several parallel lines on polyimide and to obtain areas, which are large enough for the electro-optical characterization. Figure 2.23 shows the electro-optical characteristics of the twisted nematic area obtained from unrubbed, $\mu$-rubbed polyimide. A typical transmission-voltage curve like in a conventionally rubbed system was obtained.

In Fig. 2.24, the switching characteristics of a conventional rubbed TN cell are compared with the TN areas generated by $\mu$-rubbing of pre-rubbed (buffed) polyimide. The slight increase $(0.2 \mathrm{~V})$ in threshold voltage for the $\mu$-rubbed area compared to conventional rubbing is attributed to an increase in anchoring energy and/or a decrease in surface pretilt angle. 


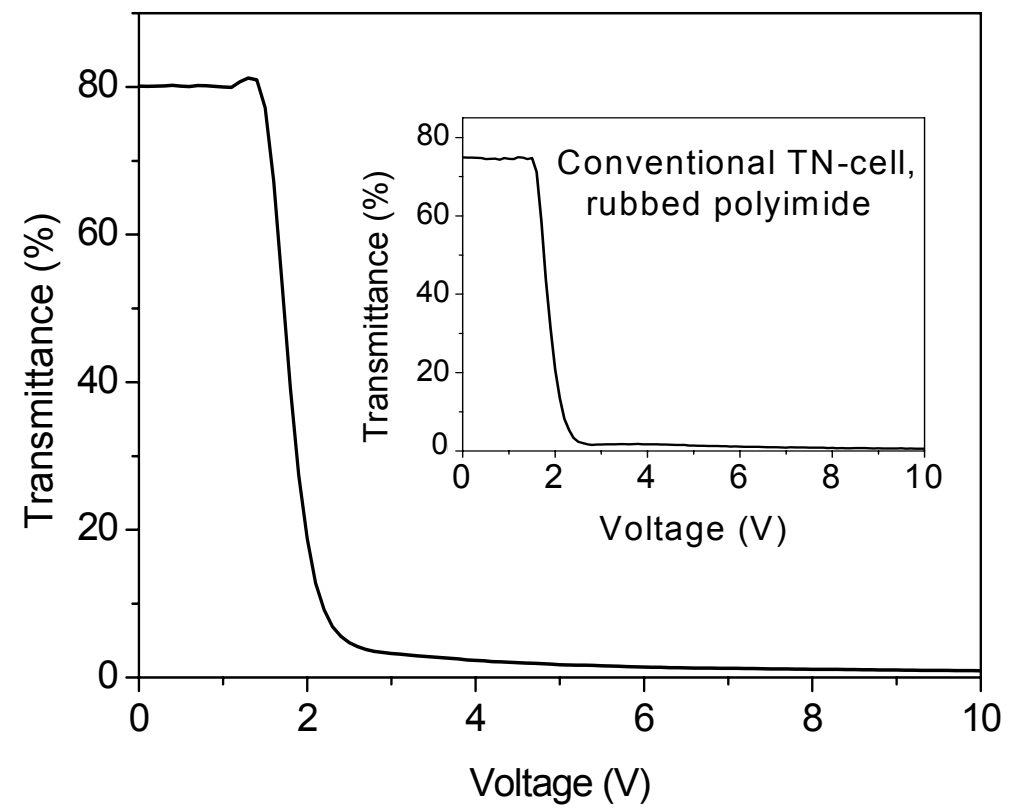

Figure 2.23: Electro-optical characteristics of TN cell created by $\mu$-rubbing process on unrubbed polyimide having a cell thickness of $18 \mu \mathrm{m}$. Inset, switching curve of a conventional TN cell at the same cell thickness.

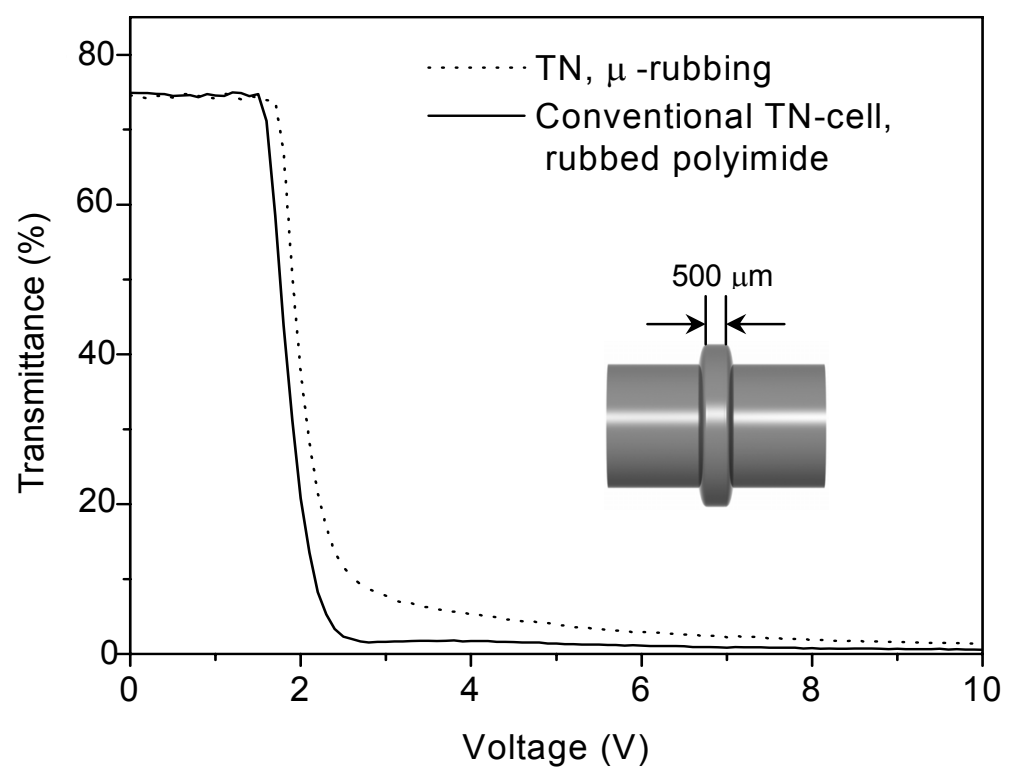

Figure 2.24: Comparison of the electro-optical characteristics of twisted nematic cell created by conventional rubbing and $\mu$-rubbing of polyimide alignment layers having a cell thickness of 18 $\mu m$. 
Analytical models ${ }^{25}$ predict a higher Fréedericksz transition voltage, which is the voltage at which the liquid crystal molecules start aligning along the field lines, with decreasing pretilt. The measured higher Fréedericksz transition voltage for the $\mu$-rubbed polyimide is therefore consistent with the observation that there is a decrease in pretilt in the $\mu$-rubbing case. Besides the small difference in the Fréedericksz transition, the $\mu$ rubbed transmission curve more slowly approaches zero transmission at high voltages (i.e. lower contrast ratio). This can be attributed to a distribution in the liquid crystal alignment along the preferred azimuthal or polar $\mu$-rubbed directions. One possible source of this phenomenon is the non-uniformity of the polished ring.

Fig. 2.25 shows the dynamic response times (measured between 10\% and $90 \%$ transmission) for the $\mathrm{TN}$ area generated from the pre-rubbing and $\mu$-rubbing process. As can be observed directly from Fig. 2.25 , there is essentially no difference between the dynamic response times of the $\mu$-rubbed samples and the conventionally rubbed samples. The off-times are essentially independent of the switching voltage and the on-times scales with the inverse of the switching voltage as expected from theory. ${ }^{22}$

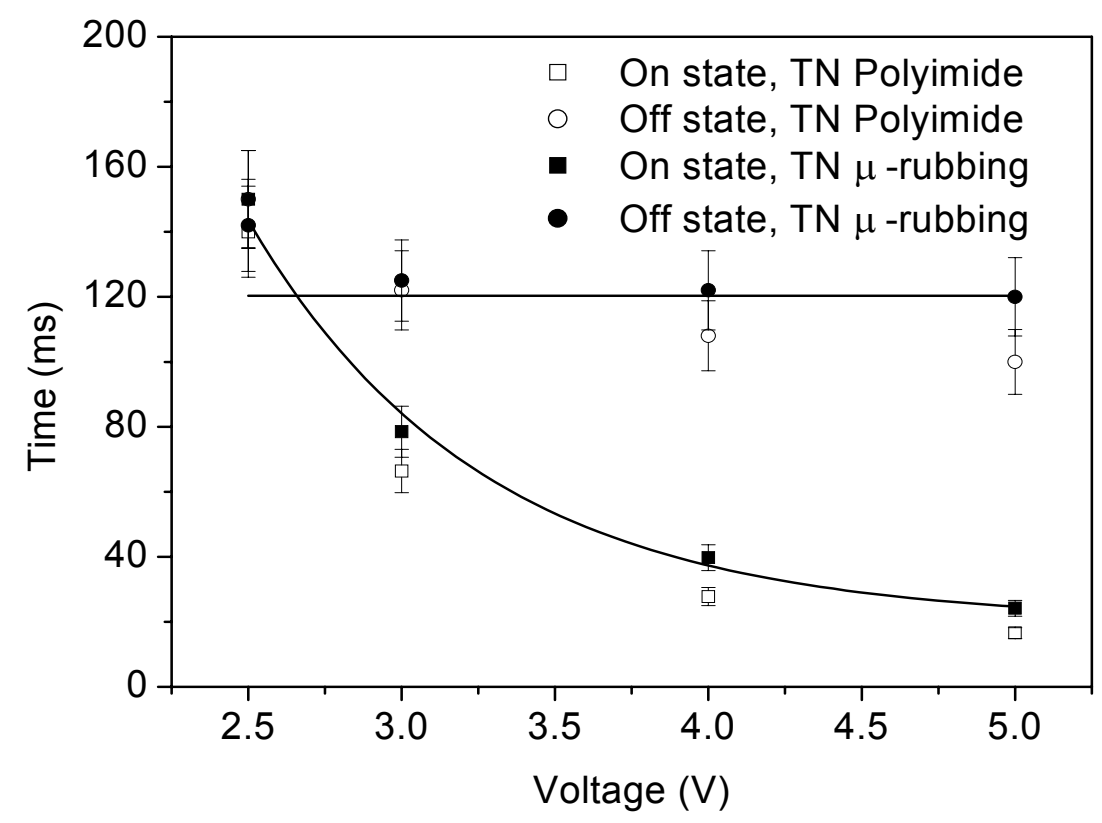

Figure 2.25: Response and relaxation times for conventional twisted nematic cell and the twisted nematic area formed by the $\mu$-rubbing, under different fields at $1000 \mathrm{~Hz}$. 
Studies were extended to measure the electro-optic performance of homogeneously aligned antiparallel cells. Anti-parallel $\mu$-rubbed cells were made with the above mentioned metallic ring with a cell thickness of $18 \mu \mathrm{m}$. The cell was placed at $45^{\circ}$ between cross polarizers for the electro-optical measurement. A typical transmission voltage curve is observed for the planar aligned sample, which oscillates due to the changes in birefringence induced by applying a voltage in a thick cell as shown in Fig. $2.26 .^{21}$

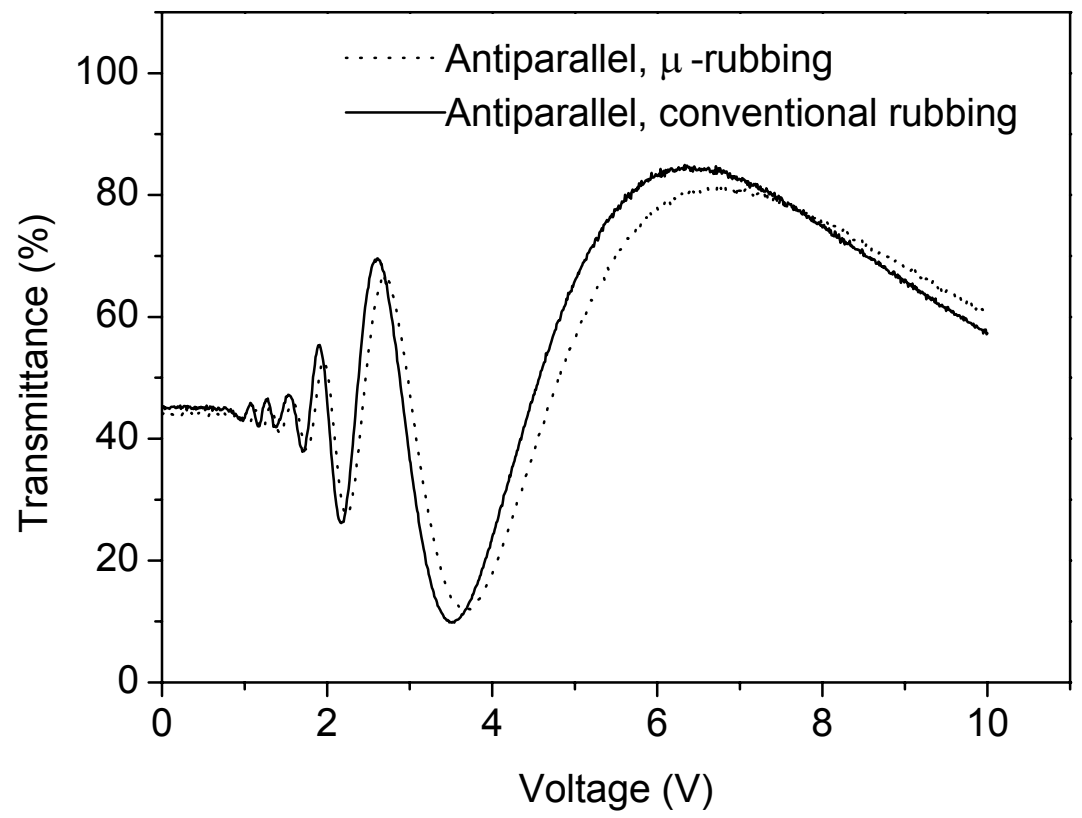

Figure 2.26: Electro-optical performance of anti-parallel cells $(18 \mu \mathrm{m}), \mu$-rubbed and prerubbed, with a driving frequency of $1000 \mathrm{~Hz}$.

Again it is observed that the $\mu$-rubbed area has a similar but slightly higher switching voltage. The same phenomenon was observed in the TN case, which was presented in Fig. 2.24.

\subsubsection{Electro-optical characteristics:- Simulations}

The transmission-voltage curve of the conventional (buffed) twisted nematic cells and $\mu$ rubbed twisted nematic cells were simulated using extended Jones matrix method as shown in Fig. 2.27. Varying the pretilt angle as the input parameter in these simulations 
there is only a subtle difference in the switching behavior of the two systems. The similarity with the measured data is good with the exception of the behavior at high voltage discussed above where the $\mu$-rubbed samples require higher voltages in order to come to complete extinction of the transmitted light.

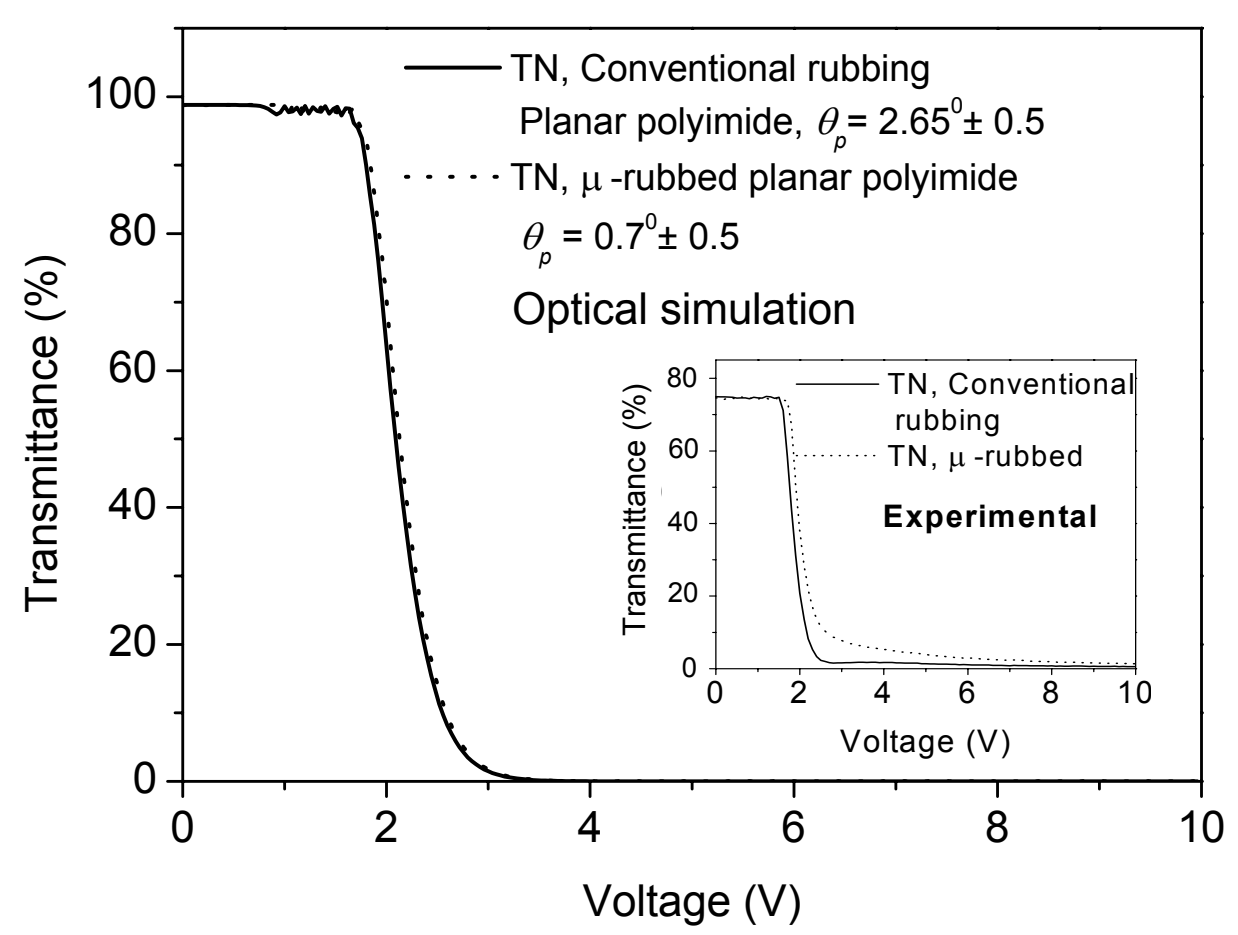

Figure 2.27: Simulated transmission-voltage curves of a conventional TN cell compared to a $\mu$ rubbed TN sample. Inset, data from the experiment.

\subsubsection{Multidomain configuration: - Planar polyimide}

To improve the viewing angle characteristics of TN displays, an attempt was made to fabricate multidomain structures using the $\mu$-rubbing technique. Figure 2.28 shows the experimental set up used for the fabrication of the multidomain cell. The unrubbed polyimide was $\mu$-rubbed in such a way that neighboring lines are rubbed in opposite directions and touching each other. Liquid crystal cells were constructed with two substrates having parallel recorded micro-patterns placed orthogonal to each other and 5 $\mu \mathrm{m}$ cell gap was used. 


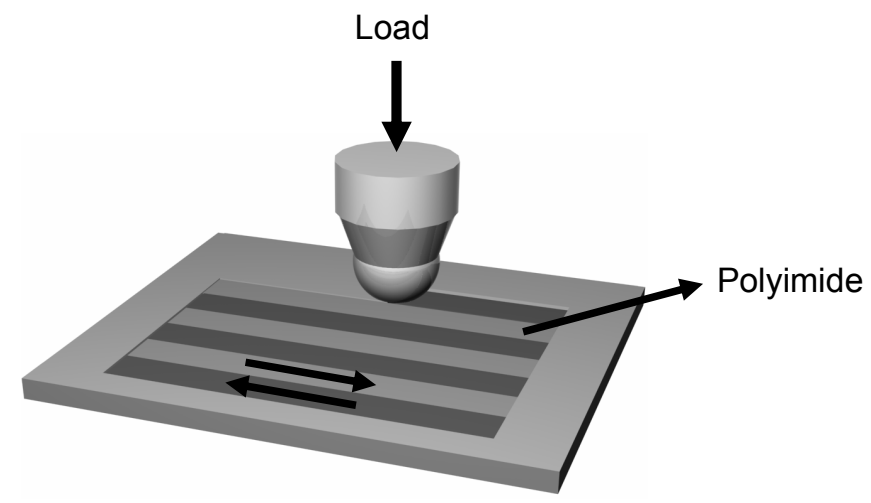

Figure 2.28: Illustration of experimental setup for the $\mu$-rubbing process, the arrows show the direction of $\mu$-rubbing.

Optical microscopy images were taken from 0 to $5 \mathrm{~V}$ (Fig. 2.29) and from 5 to 0 V (Fig. 2.30). The optical micrographs illustrates that a stable multidomain structure is not formed in both case.
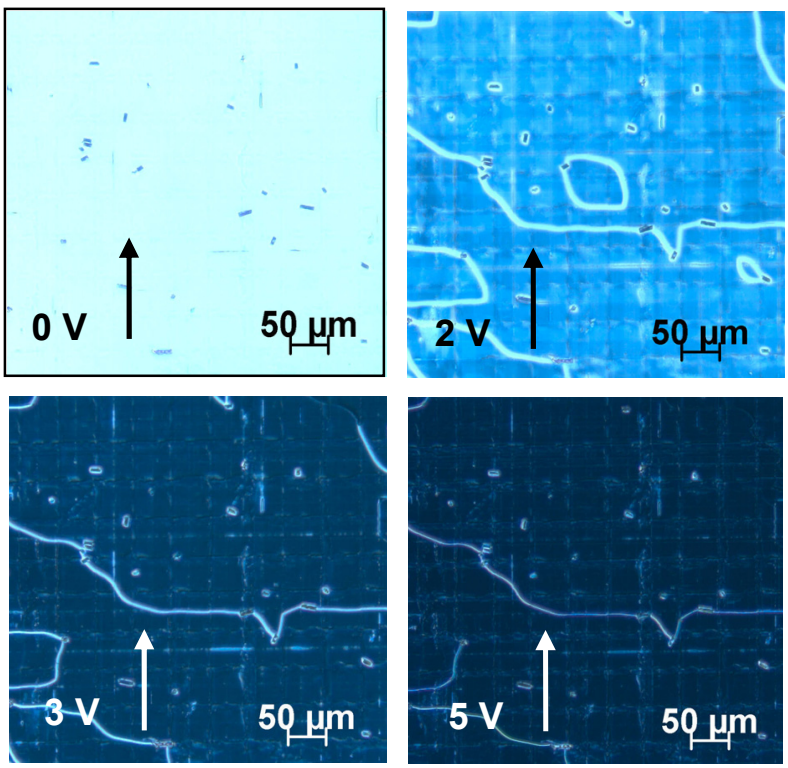

Figure 2.29: Optical microscopy images of unstable multidomain structure at different voltages $(0$ to $5 \mathrm{~V})$. 


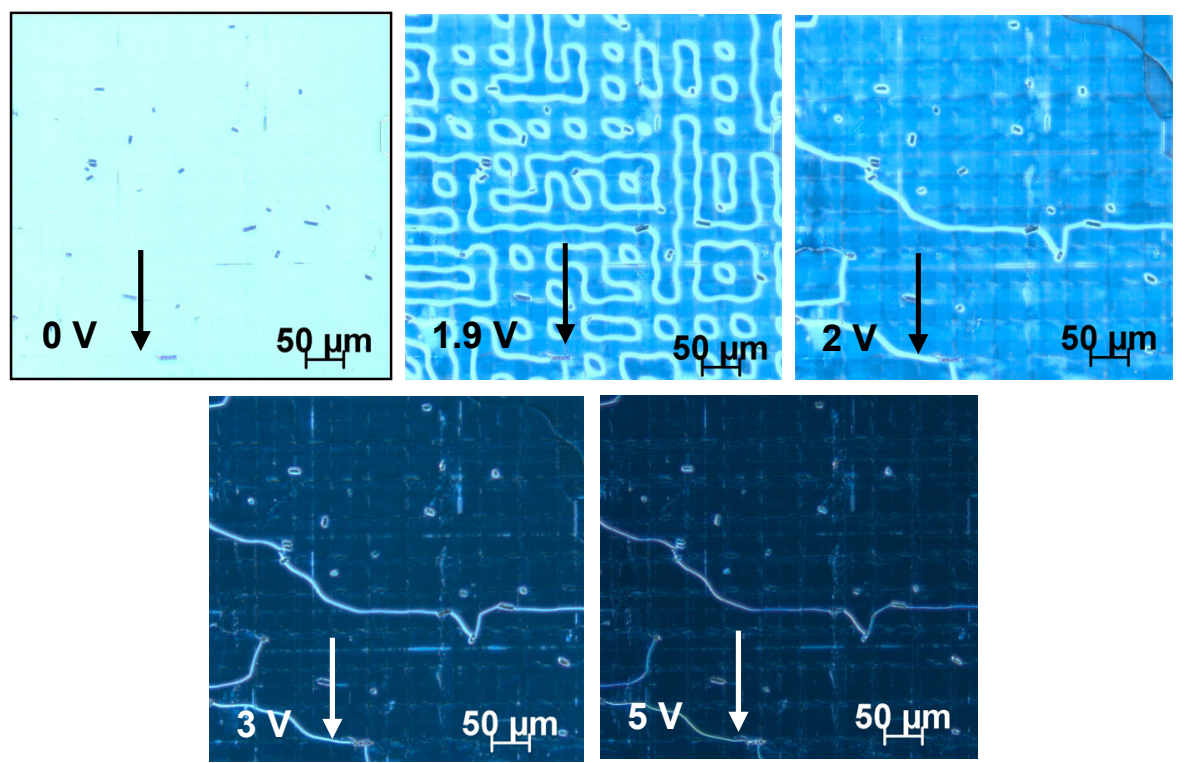

Figure 2.30: Optical microscopy images of unstable multidomain structure at different voltages (voltage decreased from 5 to $0 \mathrm{~V}$ ).

More specifically, the optical micrographs exhibit disclination lines which do not correspond to the $\mu$-rubbed regions. At first sight, the absence of a multidomain structure might seem unusual. However, as discussed earlier, $\mu$-rubbing induces planar alignment of liquid crystals and the pretilt angle of the $\mu$-rubbed areas is very small $\left(0.7^{\circ}\right)$. The required pretilt for the formation of stable domains can be estimated using a simple model proposed by Chen and co-workers. ${ }^{26}$ In this model the stability condition for multidomain formation is expressed by the following equation: $\theta_{p}{ }^{2} \geq \pi d / L$. In this equation, $\theta_{p}, d$ and $L$ represent the pretilt angle, the cell thickness and the width of a subpixel respectively. Using $L \sim 27 \mu \mathrm{m}$ and $d \sim 5 \mu \mathrm{m}$, the predicted minimum pretilt angle, $\theta_{p, \min }$, to stabilize the structure is estimated to be : $\theta_{p, \min } \sim 40^{\circ}$. In other words, a very high pretilt angle is required to stabilize the four-domain structure, which explains the absence of a multidomain structure in the $\mu$-rubbed cells (see Fig. 2.30).

In this chapter the $\mu$-rubbing of planar polyimide is presented and experimental data is compared with theoretical simulations. An excellent agreement is observed 
between theory and experiments. Also it was shown that the $\mu$-rubbing results in alignment patterns with a very low pretilt. This low pretilt probably originates from surface roughness which in turn affects the anchoring energy of the LC molecules. As a consequence of this low pretilt, the formation of a stable multidomain configuration is not possible. Of course, the impossibility to form a stable multidomain structure is prohibitive for generating wide viewing angle LCDs and, therefore, new routes are to be explored for increasing the pretilt (see the next chapters). Nevertheless, the $\mu$-rubbing of planar polyimide provides the capability to generate a wide variety of alignment patterns in liquid crystals, which are potentially useful in optical or electro-optical element such as LCDs and security devices.

\subsection{Conclusions}

The $\mu$-rubbing of polyimide alignment layers with a metallic object (sphere, ring) was investigated. Planar polyimides were used and it was found that a wide range of alignment patterns (planar, TN) could be obtained. The electro-optical behavior of the twisted nematic $\mu$-rubbed regions was investigated both experimentally and theoretically and it was found that the switching behavior hardly deviates from conventional (buffed) twisted nematic cells. A special emphasis was devoted to the evaluation of the pretilt of the $\mu$-rubbed regions and it was found that a pretilt of $0.7^{\circ}$ was generated. The formation of disclination lines and transmittance between different regions are simulated using 2 dimMOS program and verified with the experimental observation. In accordance with theoretical expectations, it was found that the pretilt is too small for the generation of multidomain LCDs with a wide viewing angle. Nevertheless, it was illustrated that $\mu$ rubbing is a versatile method for obtaining alignment patterns with dimension in the range of pixels. 


\subsection{References}

[1] J. M. Geary, J. W. Goodby, A. R. Kmetz, J. S. Patel, J. Appl. Phys. 62, 10 (1987).

[2] S. W. Depp, W. E. Howard, Sci. Am. 266, 40 (1993).

[3] S. Musa, Sci. Am. 277, 87. (1997).

[4] M. Schadt, W. J. Helfrich, Appl. Phys. Lett. 18, 127 (1971).

[5] N. A. J. M. van Aerle, J. Soc. Inf. Display 2, 41 (1994).

[6] A. Fukuro, K. Sawahata, T. Sato, H. Endo, SID International symposium, XXXI, 434 (2000).

[7] M. Neill, S. M. Kelly, J. Phys. D: Appl. Phys. 33, R67 (2000).

[8] M. Schadt, H. Seiberle, A. Schuster, Nature 381, 212 (1996).

[9] A. T. Ionescu, R. Barberi, M. Giocondo, M. Lovace, A. L. A. Ionescu, Phys. Rev. E 58, 1967 (1998).

[10] R.Yamaguchi, Y. Goto, S. Sato, Jpn. J. Appl. Phys. 41, L889 (2002).

[11] P. Chaudhari et al, Nature 411, 56 (2001).

[12] A. Kumar, N. L. Abbott, H. A. Biebuyck, G. M. Whitesides, Acc. Chem. Res. 28, 219 (1995).

[13] J. L. Wilbur, A. Kumar, E. Kim, G. M. Whitesides, Adv. Mater. 6, 600 (1994).

[14] H. T. A. Wilderbeek, F. J. A. der Meer, K. Feldman, D. J. Broer, C. W. M. Bastiaansen, Adv. Mater. 14, 655 (2002).

[15] G. P. Sinha, C. Rosenblatt, L. V. Mirantsev, Phys. Rev. E 65, 041718 (2002).

[16] B. Wen, M. P. Mahajan, C. Rosenblatt, Appl. Phys. Lett. 76, 1240 (2000).

[17] J. H. Kim, M.Yoneya, H.Yokoyama, Nature 420, 159 (2002).

[18] P. G. de Gennes and J. Prost, The physics of Liquid Crystals, Oxford University Press (1994).

[19] M. E. Becker, H. Wohler, M. Kamm and J. Kreis, Proceedings of SID 96, 596 (1996).

[20] 2dimMOS, www.autronic-melchers.com.

[21] P. Yeh, L. Gu, Optics of liquid crystal displays, John Wiley \& Sons, New York, Chapter 8 (1999). 
[22] B. L. V. Horn, H. H. Winter, Appl. Opt. 40, 2089 (2001).

[23] I. C. Khoo, F. Simoni, Physics of liquid crystalline materials, Gorden \& Breach Science Publishers, Philadelphia, Chapter XII (1988).

[24] L. M. Blinov, V. G. Chigrinov, Electro-optic effects in liquid crystal materials, Springer-Venlag, New York, Chapter 4 (1996).

[25] J. Nehring, A. R. Kmetz, T. J. Scheffer, J. Appl. Phys., 47, 850 (1976).

[26] J. Chen, P. J. Bos, D. L. Johnson, D. R. Bryant, J. Li, S. H. Jamal, J. R. Kelly, J. Appl. Phys. 80, 1985 (1996). 


\section{Chapter 3}

\section{Microrubbing of Homeotropic Polyimide*}

\subsection{Introduction}

An accurate control of the pretilt angle of liquid crystals in an LCD is a prerequisite for the fabrication of defect free displays i.e. the formation of LC domains exhibiting reverse twist and tilt is avoided. Also, pretilt of LCs is required to achieve the reorientation of the LCs in the same direction upon application of an electrical field. ${ }^{1,2}$ In conventional LCDs, the rubbing of polyimides with a velvet cloth is frequently employed to achieve molecular orientation of the LC directors in the rubbing direction. In such a rubbed polyimide film, the angle that the liquid crystal director makes with the surface, further denoted as pretilt, is usually less than $\pm 5^{\circ} .{ }^{3,4}$ For planar alignment, the nature of the polyimide and the parameters associated with rubbing, like the cloth and the rubbing strength, are instrumental in controlling the pretilt angle of the LC director.

Several methods were reported to obtain higher pretilt angles in LCs. For instance, the pretilt of polyimide films which normally induce a homeotropic alignment (pretilt $=90^{\circ}$ ) was converted to a value of $\sim 55^{\circ}$ by solvent treatment. ${ }^{5}$ In another case, a large pretilt $\left(50^{\circ}-85^{\circ}\right)$ was observed in homeotropic polyimides with a relatively rigid backbone structure by varying the rubbing pressure. ${ }^{6}$ Also a pretilt of $15^{\circ}$ was achieved for special polyimides with trifluoromethyl moieties attached to the benzene rings in combination with physically rubbing the surface. ${ }^{7}$ These alignment layers with a high and uniform pretilt were used for LCDs with monodomain structures having fast switching characteristics.

*This chapter is partly reproduced from: S. Varghese, G. P. Crawford, C. W. M. Bastiaansen, D. K. G de Boer, D. J. Broer, Mol. Cryst. Liq. Cryst. 429, 55 (2005). 
As discussed previously, different methods were explored in the past to generate multidomain LCDs (see Chapter 1). In these multidomain LCDs pretilt control is vital and there are different methods available to control the pretilt angle of the LC molecules. For instance, in the case of photoalignment with linearly polarized light, the pretilt angle is controlled by the oblique irradiation of these materials. The polyimide that was selected for these experiments is known to provide planar alignment after velvet rubbing with low, though controlled, pretilt angles. The pretilt angle achieved was shown to be too low for obtaining stable multidomain structures and it was suggested that a far higher pretilt is required for inducing stable multidomain in zero fields.

In the previous chapter $\mu$-rubbing was performed on unrubbed and pre-rubbed (buffed) polyimide, which resulted in planar alignment with low surface pretilt angle $\left(<1^{\circ}\right)$. In this chapter, a new route is explored to induce a high pretilt in $\mu$-rubbed alignment layers. More specifically, it is attempted to generate a high pretilt angle in $\mu$ rubbed patterns using homeotropic polyimide alignment layers (instead of planar alignment layers). Homeotropic polyimides are polyimides that, in the absence of any rubbing treatment orient the liquid crystals with their director perpendicular to the surface. In order to achieve this, most often these are polyimides modified with low energetic moieties that tend to direct perpendicular to the surface to minimize surface energy. This orientation is taken over in an epitaxial manner by the liquid crystal that is brought into contact with this surface.

\subsection{Experimental}

\subsubsection{Materials and sample preparation}

A polyimide precursor, AL-75114 (JSR electronics) was used to create a homeotropic orientation layer on indium tin oxide (ITO) coated glass substrates. Spin coating of the polyimide precursor was performed using a Karl Süss CT 62 spin coater ( 5 seconds at $1000 \mathrm{rpm}, 40$ seconds at $5000 \mathrm{rpm}$ ) on the ITO side of the $25 \times 25 \mathrm{~mm}$ substrates. After spin coating, the substrates were preheated to $100{ }^{\circ} \mathrm{C}$ for 10 minutes. The samples were 
then imidized at $180{ }^{\circ} \mathrm{C}$ for 90 minutes in a vacuum oven. The thickness of the polyimide coating was $\sim 150 \mathrm{~nm}$

The $\mu$-rubbing of polyimide substrates was carried out with a mechanical device using a $1 \mathrm{~mm}$ diameter metallic sphere as shown in Chapter 2, Fig. 2.1a. Patterns were recorded by writing at a constant load $(150 \mathrm{~g})$ and velocity $(10 \mathrm{~mm} / \mathrm{s})$ at room temperature. Liquid crystal cells were constructed with the $\mu$-rubbing direction on the top and bottom surface oriented orthogonally and secured with UV curable glue (Norland UV Sealant 91). The cell gap was controlled by $5 \mu \mathrm{m}$ spacers. Liquid crystal E7 from Merck (Darmstadt, Germany) $\left(\mathrm{T}_{\mathrm{NI}}=58{ }^{\circ} \mathrm{C}, \rho=1.06 \mathrm{~g} / \mathrm{cm}^{3}, \varepsilon_{/}=19\right.$ and $\varepsilon_{\perp}=5.2$, and $\triangle \mathrm{n}=0.2253$ ) was used in the cells. The cells are filled with liquid crystal material E7, by capillary action at $80{ }^{\circ} \mathrm{C}$, which is $\sim 20{ }^{\circ} \mathrm{C}$ above the nematic-isotropic transition temperature of the LC.

\subsubsection{Characterization}

The $\mu$-patterns in the polyimide were investigated by atomic force microscopy (AFM) (Nanoscope IIIa, Digital Instruments, Santa Barbara, California) equipped with conventional $\mathrm{Si}_{3} \mathrm{~N}_{4}$ cantilevers, and the measurements were performed in tapping mode. Optical micrographs of the liquid crystal configurations were recorded with a polarized light microscope (Zeiss LM Axioplan) equipped with a digital camera. The cell thickness was determined from the wavelength dependent interference pattern generated by a UVvisible spectroscopy (Shimadzu UV-3102 PC). The electro-optical characteristics were investigated using the DMS 703 display measuring system (autronic-Melchers GmbH). A square AC signal of $1 \mathrm{kHz}$ was used to drive the cells for the dynamic response measurements.

\subsubsection{Line scanning}

Line scanning was performed using a Lab view computer program. An area was selected from the optical microscopy pictures, which contains different configurations (planar, 
TN). A line was drawn on these optical microscopy images and the intensity vs. position is plotted.

\subsubsection{Pretilt angle measurement}

Pretilt measurements were carried out using Autronic, TBA 107 that employs the crystal rotation method. Both antiparallel cells with a thickness of $18 \mu \mathrm{m}$ and twisted nematic cells with a thickness of $5 \mu \mathrm{m}$ were constructed. The details of the measurement and setup are described in Chapter 2 (section 2.3.6).

\subsection{Results and discussion}

\subsubsection{Morphology of patterns}

Several adjacent parallel lines were recorded and a constant spacing between $\mu$-rubbed lines was used. The surface roughness of the metallic sphere was found to be $8 \pm 2 \mathrm{~nm}$ that was measured using AFM. Figure 3.1 shows the AFM image of the $\mu$-rubbed area at a load of $150 \mathrm{~g}$. The polyimide layer has a thickness of $150 \mathrm{~nm}$ and a line width of 47 $\mu \mathrm{m}$ was observed.

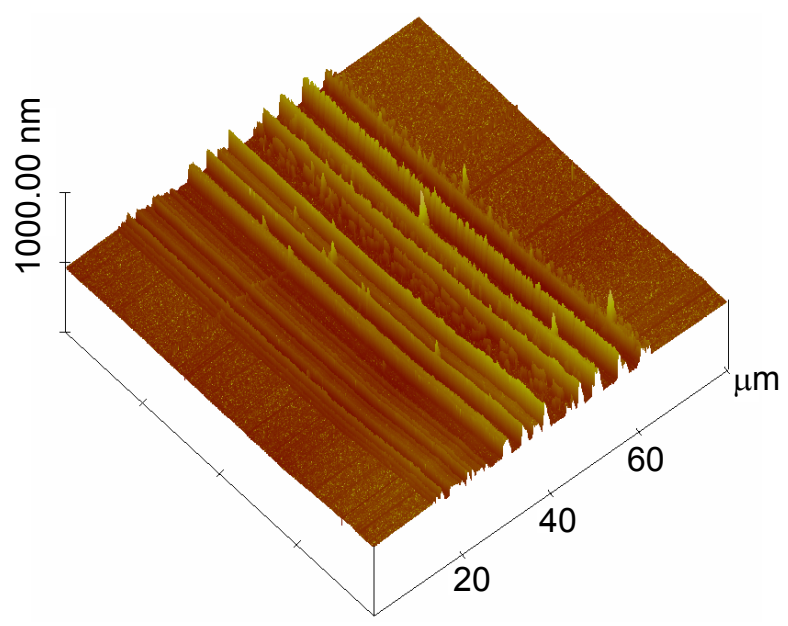

Figure 3.1: Atomic force microscopy image of the $\mu$-rubbed homeotropic polyimide at a load of $150 \mathrm{gm}$. 
The surface roughness of the polyimide after $\mu$-rubbing was found to be $40 \pm 2 \mathrm{~nm}$, which is five times larger than the surface roughness of the metallic sphere.

The width of the $\mu$-rubbed line depends on polyimide film thickness, sphere diameter and the applied load. The $\mu$-rubbing was performed at different loads and it was found that the line width increases with applied load as depicted in Fig.3.2. The same results were observed in Chapter 2, Fig. 2.7. The average surface roughness of the polyimide layer also increases with the applied load as is shown in Fig.3.2 (inset).

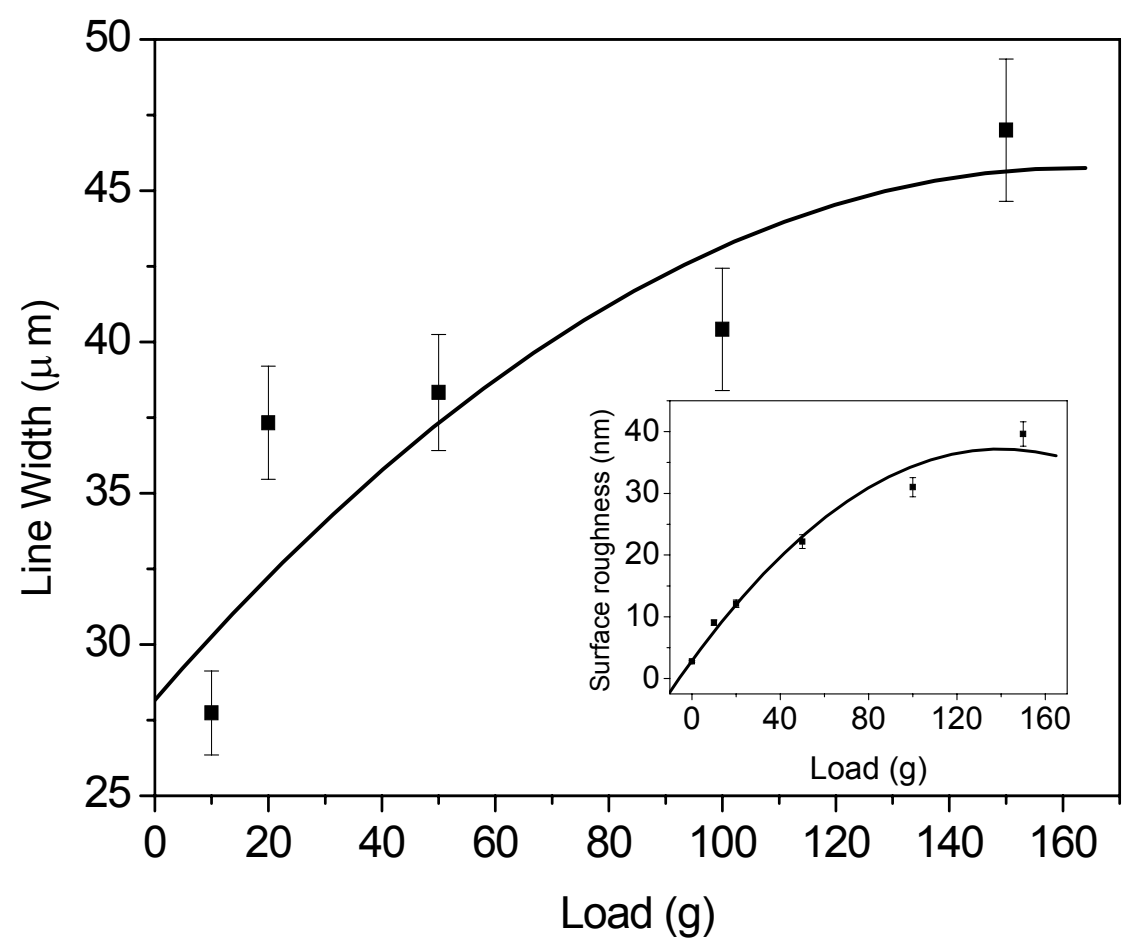

Figure 3.2: Variation of line width with applied load measured with atomic force microscopy. Inset, variation of the surface roughness for the same load.

\subsubsection{Multi-configurations}

In a first set of experiments liquid crystal cells were constructed with two $\mu$-rubbed substrates orthogonal to each other. In this particular case, the $\mu$-rubbing was found to induce planar alignment of liquid crystals. Figure 3.3 shows an optical micrograph of the 
cell viewed between crossed polarizers. A uniform alignment of LCs is observed in the $\mu$-rubbed area.

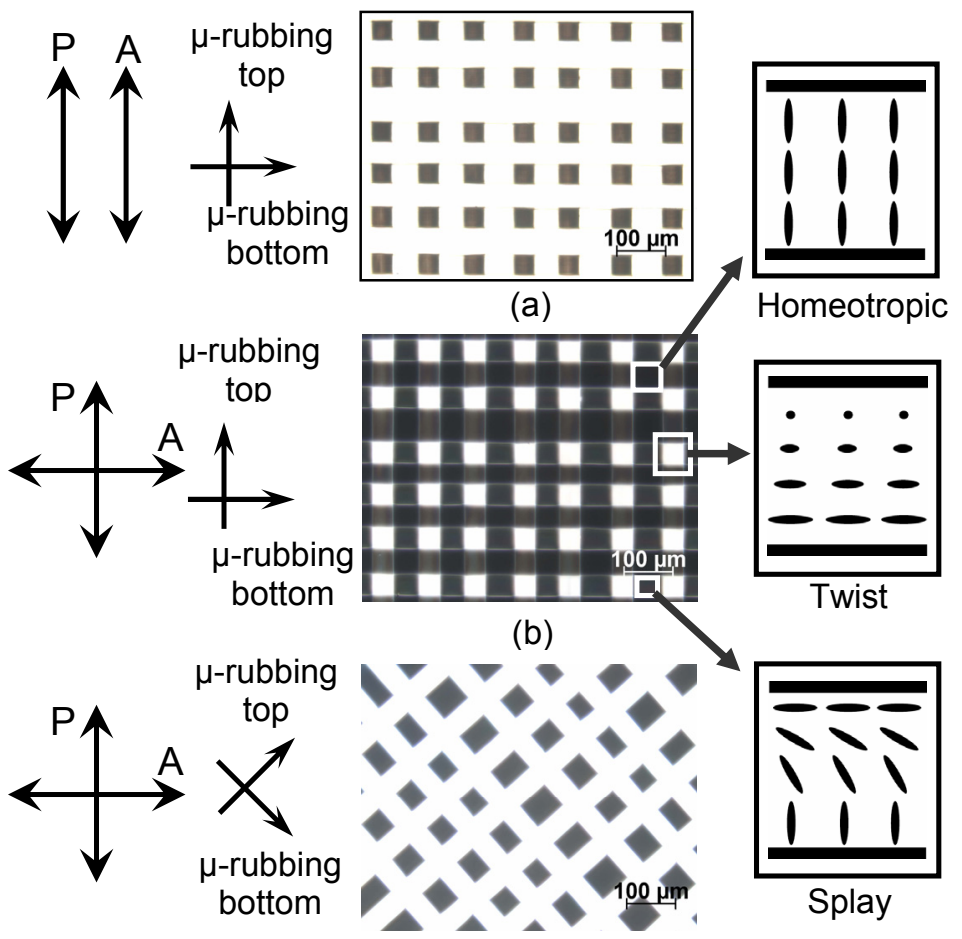

(c)

Figure 3.3: Optical micrographs of electro-optical cell with homeotropic, $\mu$-rubbed polyimide substrates on both sides (load: $150 \mathrm{~g}$ ). Line patterns mutually crossed, a) between parallel polarizers b) crossed polarizers c) Lines at $45^{\circ}$ between crossed polarizers. The respective liquid crystal configuration is also shown.

In Fig. $3 b$ three different liquid crystal configurations are found. These are compatible with the following three expected configurations: a twisted nematic configuration where the two $\mu$-rubbed areas from the top and bottom substrates intersect, a splay configuration in areas with hybrid anchoring (homeotropic polyimide on one substrate and $\mu$-rubbed polyimide on the other substrate) and a homeotropic configuration in the areas where both substrates are unrubbed. 


\subsubsection{Pretilt angle measurements}

\section{Method 1: Antiparallel cell}

The pretilt angle was measured using antiparallel cell with a cell thickness of $18 \mu \mathrm{m}$ using a crystal rotation method. ${ }^{13}$ For this measurement several parallel lines were recorded on the polyimide substrate, the top and bottom substrates are glued together in the antiparallel direction with respect to the $\mu$-rubbing. The pretilt angle for this antiparallel construction was found to be $9.8^{\circ}$ with respect to the surface.

\section{Method 2: Twisted nematic cells}

The pretilt angle was measured in a twisted nematic cell using crystal rotation method at different loads. For the pretilt angle measurements a set of parallel lines were recorded at different loads. The top and bottom substrates are crossed to obtain a twisted nematic configuration. By adopting this method a constant cell gap was achieved. Fig.3.4 shows the variation of pretilt angle with applied load.

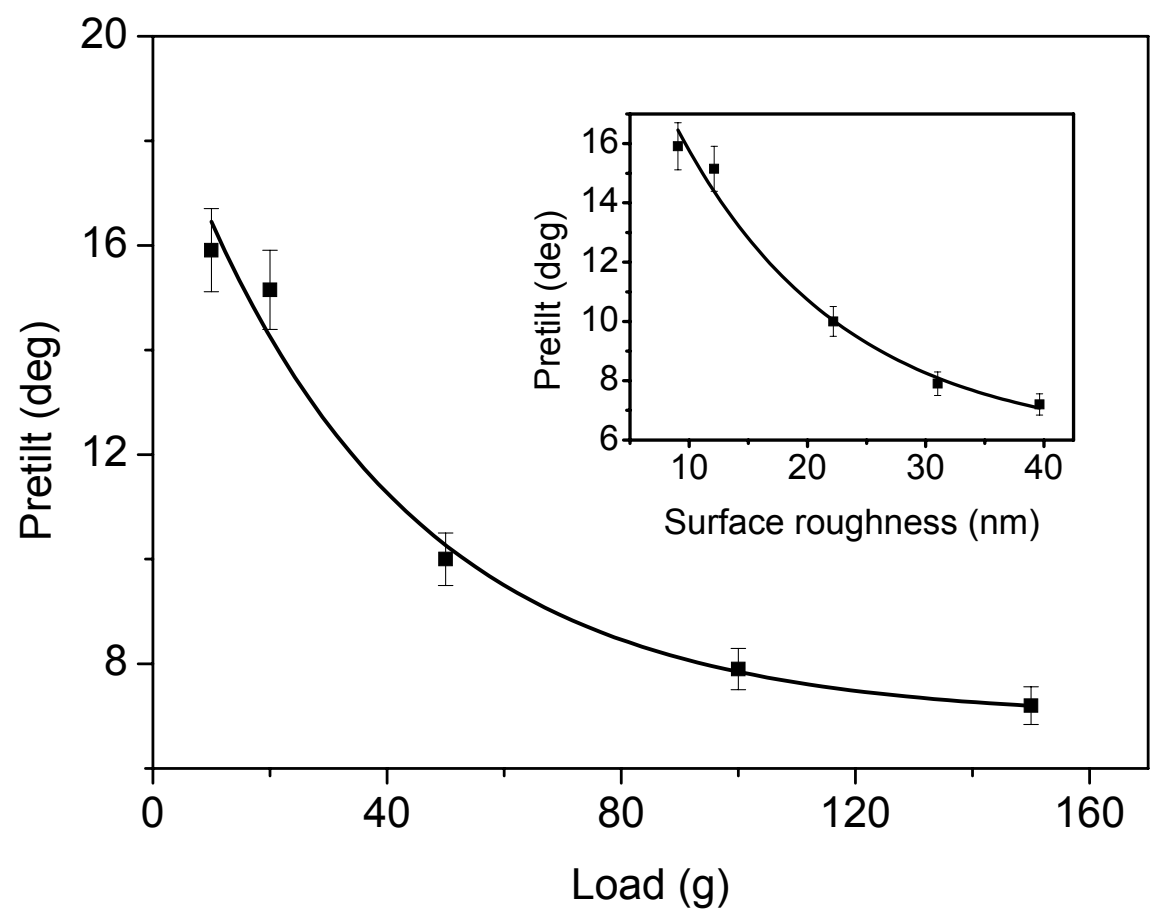

Figure 3.4: The variation of pretilt angle with applied load, inset, variation of pretilt angle with surface roughness. 
The pretilt angle is found to decrease with an increase in applied load. The pressure applied on the polyimide tends to increase the surface roughness, which in turn affects the pretilt angle. The pretilt angle measured for $150 \mathrm{~g}$ was $7.2^{\circ}$ for the TN cell (5 $\mu \mathrm{m})$ and $9.8^{\circ}$ for the antiparallel cell $(18 \mu \mathrm{m})$. This difference may be due to the difference in cell construction and the difference in cell gap. Loads below $10 \mathrm{~g}$ can result in higher pretilt, but the alignment of the LC is poor and is not good for an optical device.

The switching behavior of the $\mu$-rubbed cell (twisted nematic region) is measured by placing the cell between cross polarizers and applying a voltage from $0 \mathrm{~V} \rightarrow 5 \mathrm{~V}$. Figure 3.5 shows the optical micrographs of the cell at increasing voltage. If a smaller load was used during $\mu$-rubbing, the TN areas switch at a lower voltage. Probably, this is related to an increasing surface pretilt angle.
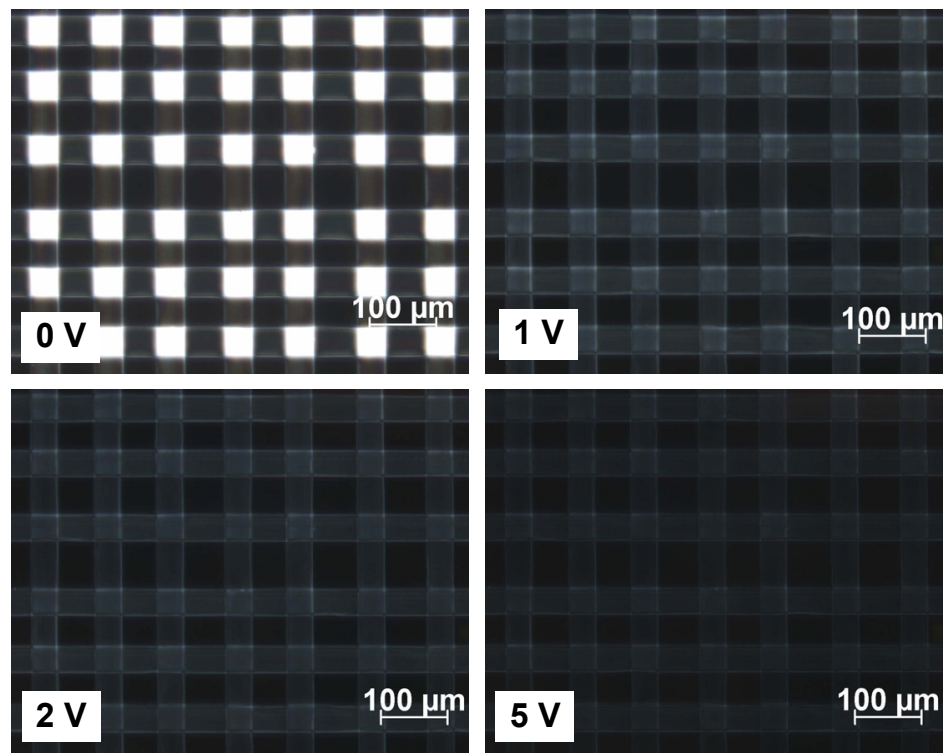

Figure 3.5: Optical micrographs of liquid crystal cell with $\mu$-rubbed homeotropic polyimide substrates (substrates crossed) between crossed polarizers at different voltages (0-5 V). 


\subsubsection{Optical simulations:- Director orientations and transmittance}

Optical simulations of the director orientations were performed using the 2dimMOS $\operatorname{program}^{14,15}$ and the transmittance was calculated using the extended Jones matrix method (see Chapter 2; section 2.2). In Fig. 3.6 a cross section of a cell with a TN area in the off-state is shown. The gray rods represent the LC directors, where the light end indicates a forward direction and the dark ends indicate a backward direction with respect to the plane of drawing. The pretilt angles used in the optical simulations are obtained from the experiment. In Fig. 3.6a the top part has uniform surface pretilt due to $\mu$ rubbing, and the bottom part is divided into three parts. The bottom left and right parts are in homeotropic state and the middle part is $\mu$-rubbed in a direction orthogonal to the top substrate, which creates a TN area (Fig.3.6a). The pretilt angle and the direction of LC molecules used in the calculation are indicated in Fig. 3.6a. On the left and right sides of the $\mathrm{TN}$ area, a splay configuration is observed (originating from the bottom substrate with a homeotropic and the top substrate with a planar alignment). These splay configuration results in a black state because their orientation direction is parallel with one of the (crossed) polarizers.

The top part of the Fig. 3.6a shows the transmission characteristics of the TN area calculated using the extended Jones matrix method. The calculated transmission characteristics are in good agreement with the experimental results obtained by the line scanning of the optical microscopy image as shown in Fig. 3.6b. The director orientations and the transmission characteristics are also calculated for the TN cell in the on-state $(5 \mathrm{~V})$. Due to the higher pretilt angle in the $\mu$-rubbed region, in an applied field all the LC molecules can easily align to homeotropic state without a clear formation of disclination lines. Only a very small increase of intensity is seen at the borders of the domains, both in the experiment and simulations. The simulated transmission characteristics are also comparable with the line scanning results as shown in Fig 3.7b. 


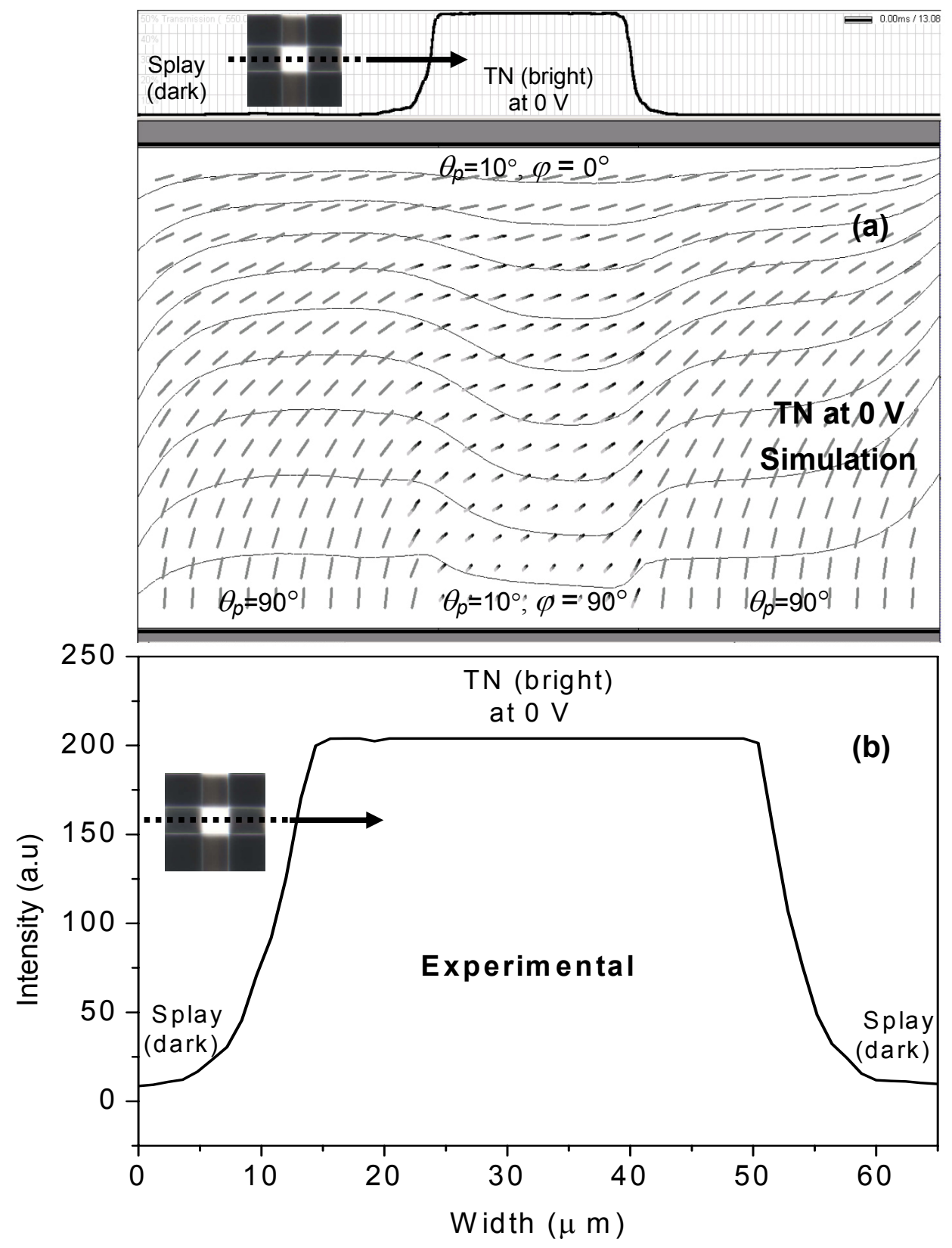

Figure 3.6: Comparison of transmittance of the TN area at $0 V$ from optical simulation (a) and experimental results (b), obtained by the line scanning of the optical micrograph shown in the inset. The director orientations from $2 \operatorname{dimMOS}$ calculation of the TN area at $0 \mathrm{~V}$ is also shown in (a). 


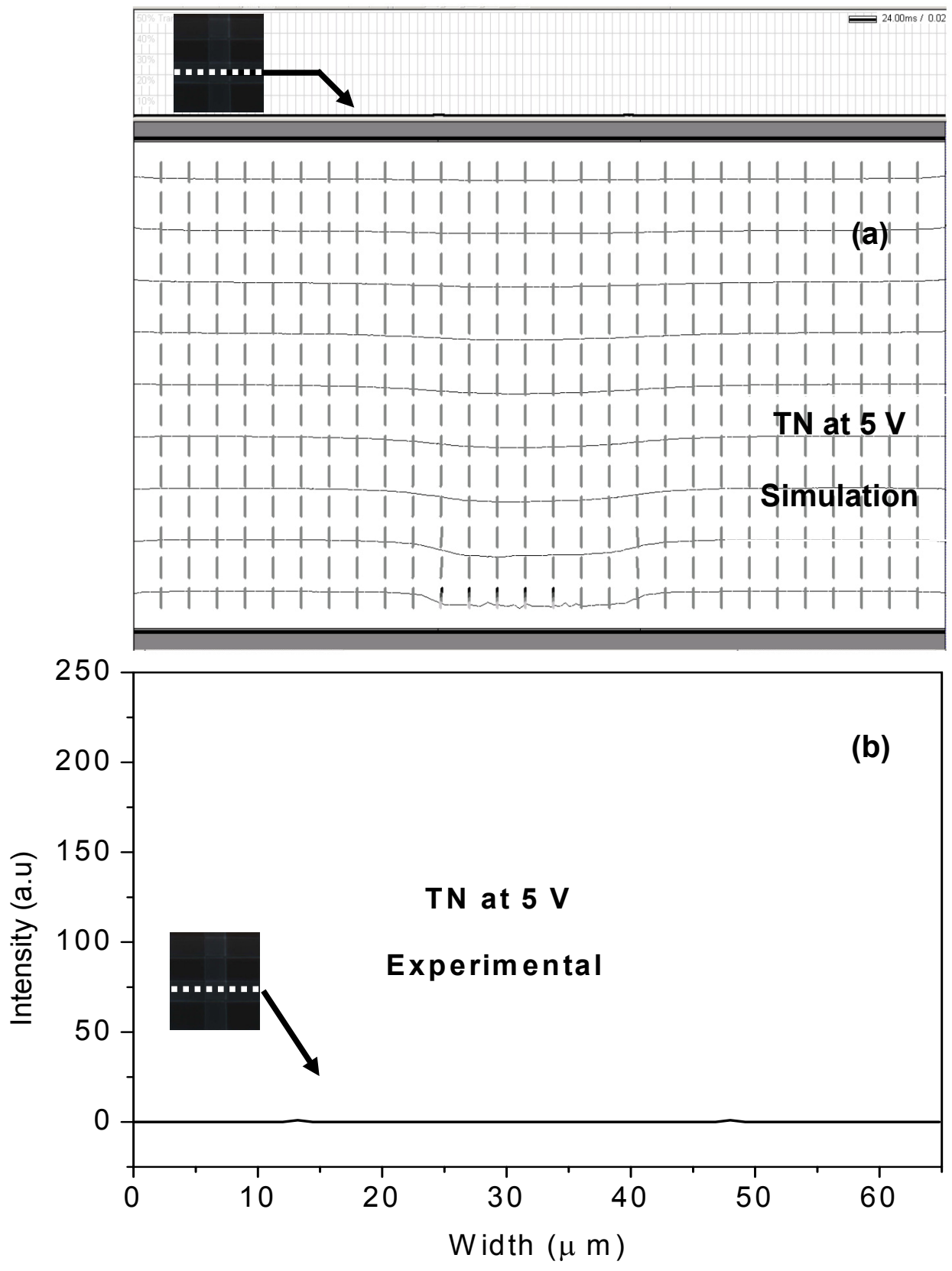

Figure 3.7: Comparison of transmittance of the TN area at $5 \mathrm{~V}$ from optical simulation (a) and experimental results (b), obtained by the line scanning of the optical micrograph shown in the inset. The director orientations from 2dimMOS calculation of the TN area at $5 \mathrm{~V}$ are also shown in (a). 
Figure 3.8a shows the optical simulation of the director orientations in the splay area at $0 \mathrm{~V}$. The pretilt and the directions used in the calculation are indicated in Fig. 3.8a. Here the top substrate is unrubbed and bottom middle part is $\mu$-rubbed, creating a splay configuration. The difference in direction of LC molecule orientation in the unrubbed and in the $\mu$-rubbed area leads to the formation of disclination lines as observed in Fig.3.8a at 0 V. The calculated transmission characteristics of the splay area are also shown in the top of Fig. 3.8a. This calculated transmission characteristics is comparable with the experimental results obtained by the line scanning of the optical microscopy images as shown in Fig. 3.8b. There is some difference in simulation and experiment in the light transmission at the splay area at $0 \mathrm{~V}$. The middle part of the transmittance in the simulation is flat and in the experiment (line scanning) the light transmission is slightly higher. This may be due to the fact that the transmission in this area is more sensitive to small miss-orientation of the polarizers.

In Fig. 3.9a the director orientation in the splay state at $5 \mathrm{~V}$ is shown. All the directors are in the homeotropic state which gives rise to a black state. The calculated transmission is also plotted above and is in good agreement with the experimental line scanning results as shown in Fig.3.9b. 


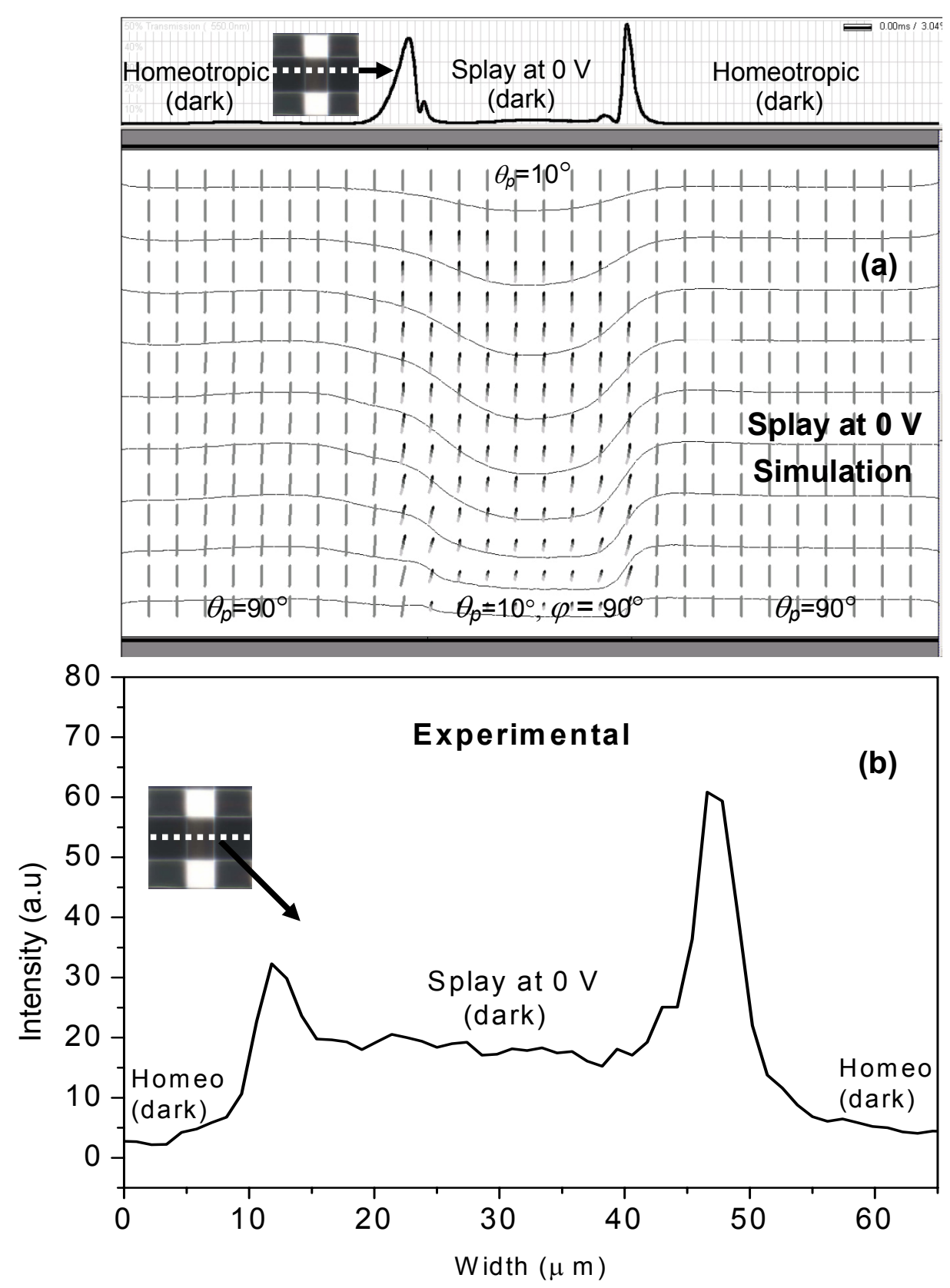

Figure 3.8: Comparison of transmittance of the splay area at $0 \mathrm{~V}$ from optical simulation (a) and experimental results (b), obtained by the line scanning of the optical micrograph shown in the inset. The director orientations from 2dimMOS calculation of the splay area at $0 \mathrm{~V}$ are also shown. 

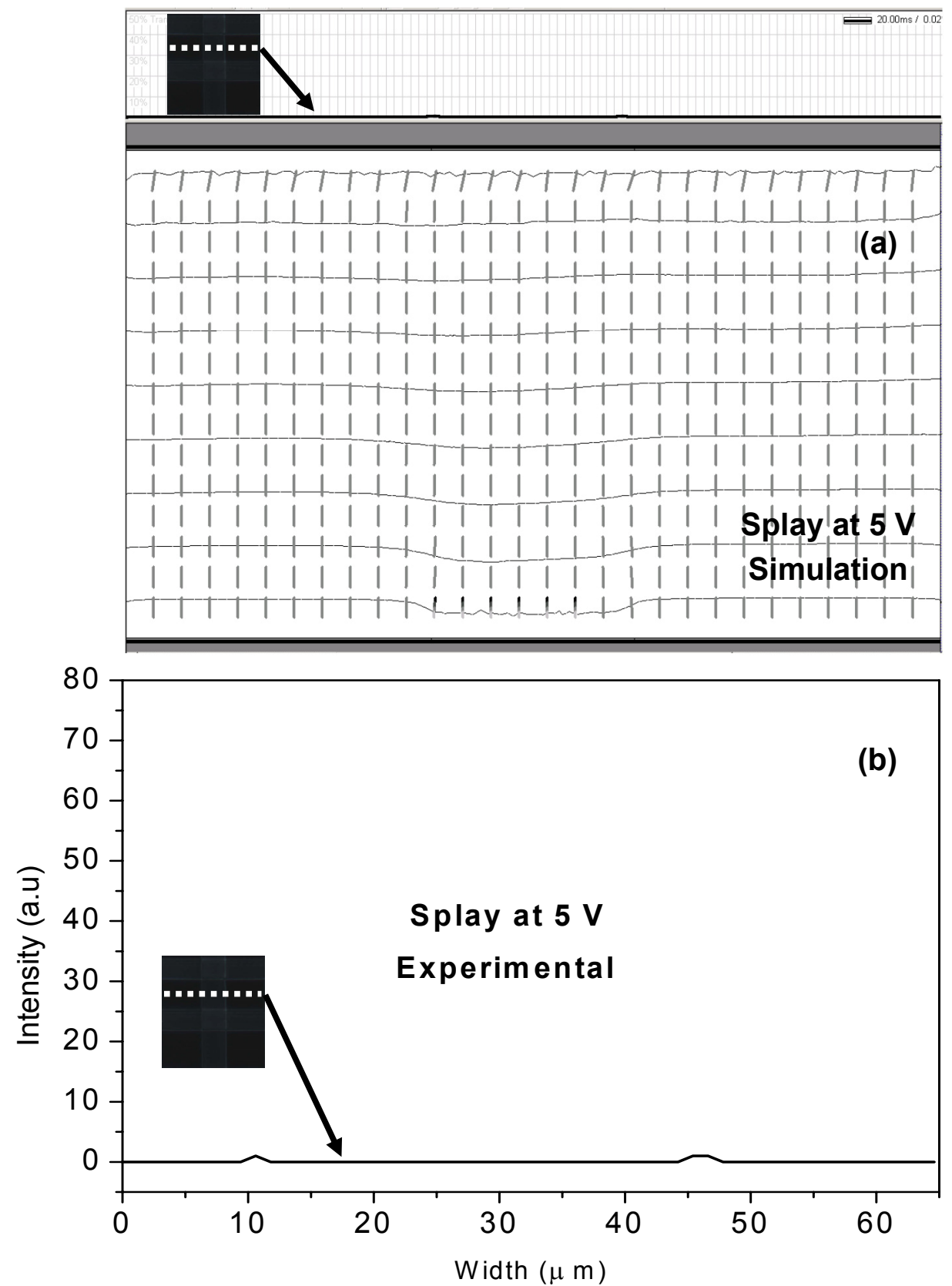

Figure 3.9: Comparison of transmittance of the splay area at $5 \mathrm{~V}$ from optical simulation (a) and experimental results (b), obtained by the line scanning of the optical micrograph shown in the inset. The director orientations from 2dimMOS calculation of the splay area at $5 \mathrm{~V}$ are also shown. 


\subsubsection{Electro-optical characteristics}

The switching characteristics of $\mathrm{TN}$ areas produced at different loads were also measured. Figure 3.10 shows the influence of applied load during $\mu$-rubbing on transmission-voltage curve. At a low load $(10 \mathrm{~g})$ the $\mathrm{TN}$ area switches faster in comparison to $\mathrm{TN}$ areas recorded at a high load. This observed behavior is due to a difference in surface pretilt angle. For $10 \mathrm{~g}$ load a surface pretilt of $16^{\circ}$ is observed and it switches faster in comparison to $150 \mathrm{~g}$ load with a pretilt angle of $7.2^{\circ}$.

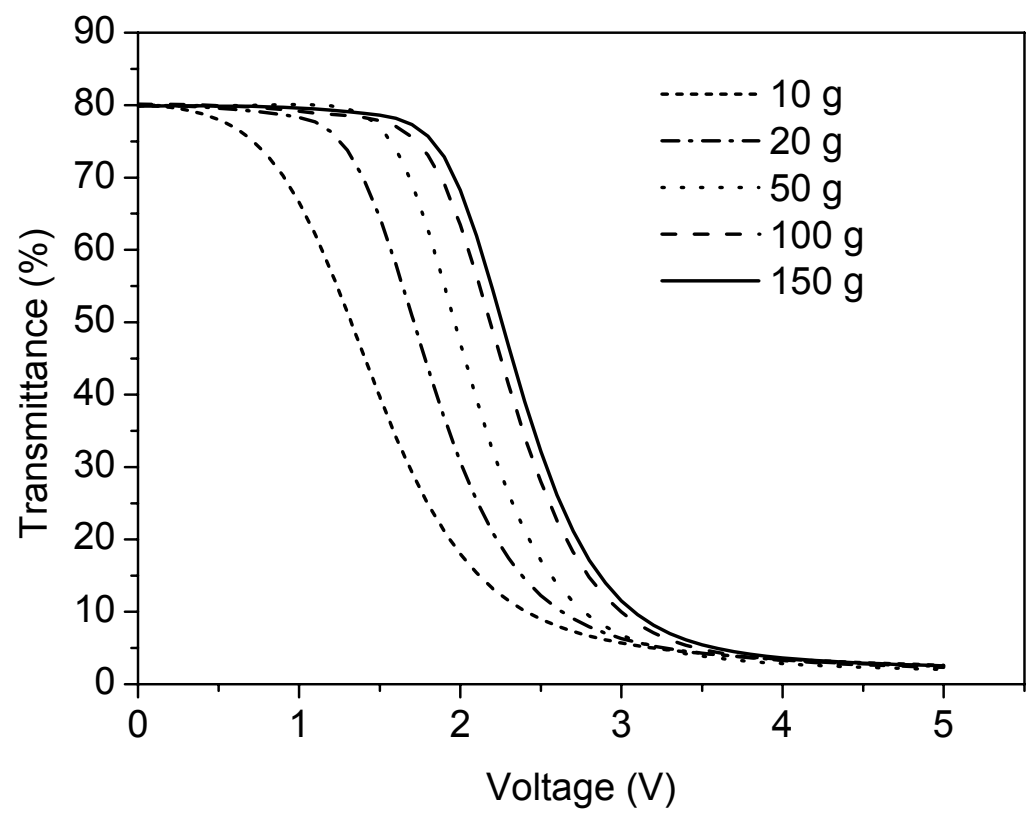

Figure 3.10: Electro-optical characteristics of TN cell created by $\mu$-rubbing on homeotropic orientation layers at different loads having a cell thickness of $18 \mu \mathrm{m}$.

Figure 3.11 shows the dynamic response time (measured between $10 \%$ and $90 \%$ transmission) for the $\mathrm{TN}$ area produced by $\mu$-rubbing of homeotropic polyimide at different loads. It is found that for the on-state switching time scales with the inverse of voltage ${ }^{16}$ and there is slight increase in the response time with increasing load. Figure 3.12 shows the relaxation times (off-state) at different loads, it is obvious from the graph that time scales involved in the off-state are essentially identical. 


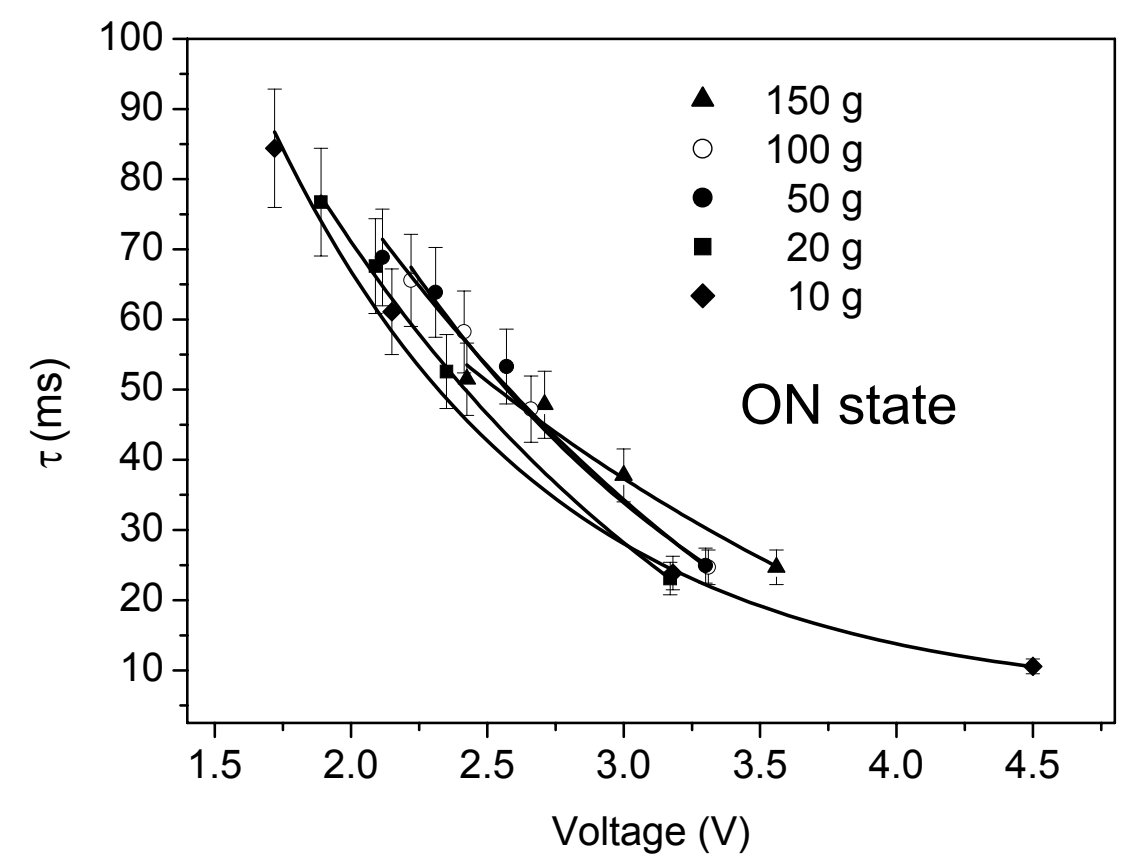

Figure 3.11: Response time for TN area formed by the $\mu$-rubbing of homeotropic polyimide at different voltages.

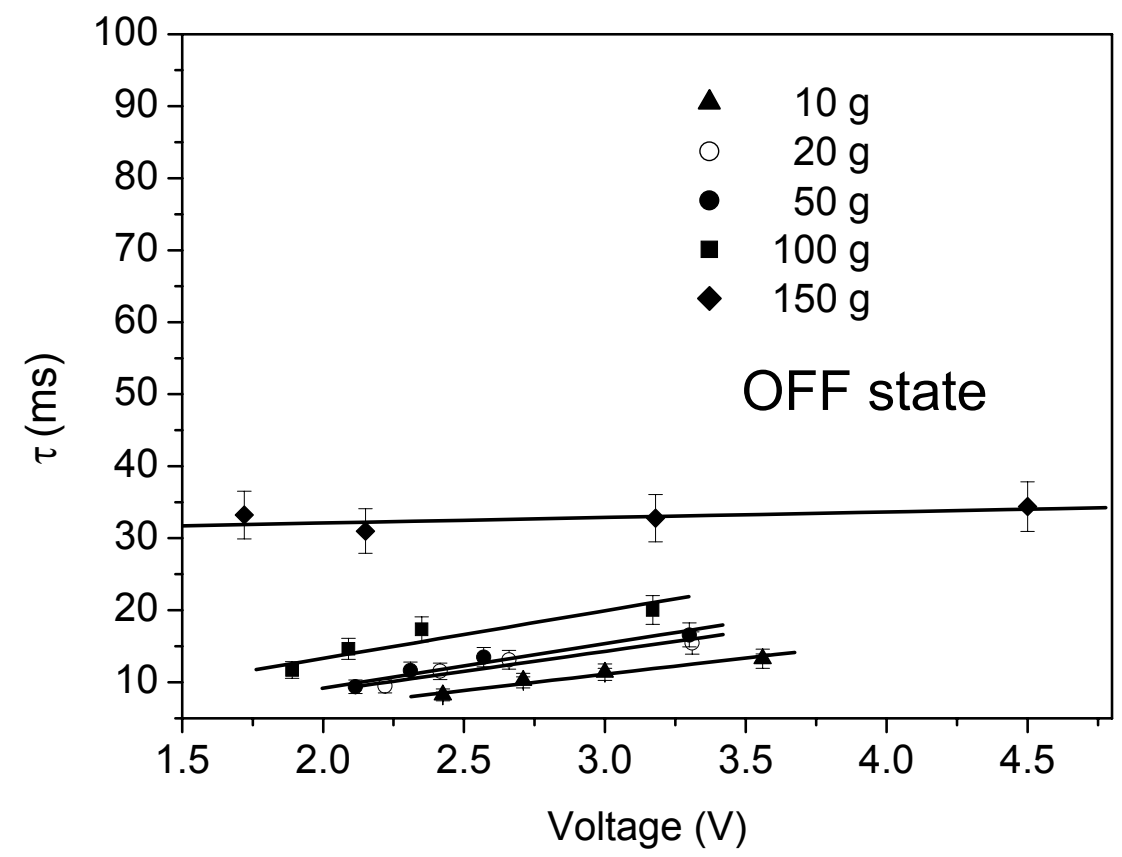

Figure 3.12: Relaxation time for TN area formed by the $\mu$-rubbing on homeotropic polyimide at different voltages. 


\subsubsection{Electro-optical characteristics: Simulations}

The electro-optical characteristics are calculated using the extended Jones matrix method $^{17}$ and compared with the experimental results. Figure 3.13 shows the comparison of the optical simulations with experimental results for conventionally rubbed (buffed) polyimide with a pretilt angle $2.65^{\circ}$ and $\mu$-rubbed polyimide with a pretilt angle of $9.8^{\circ}$. The experimental results are shown in Fig.3.14, The simulations and the experimental results are in good agreement. The near thresholdless nature of the $\mu$-rubbed region indicates that there is a high pretilt for the twisted nematic area. This reduction of the switching threshold with increasing pretilt is consistent with models correlating pretilt to threshold voltages. ${ }^{18}$

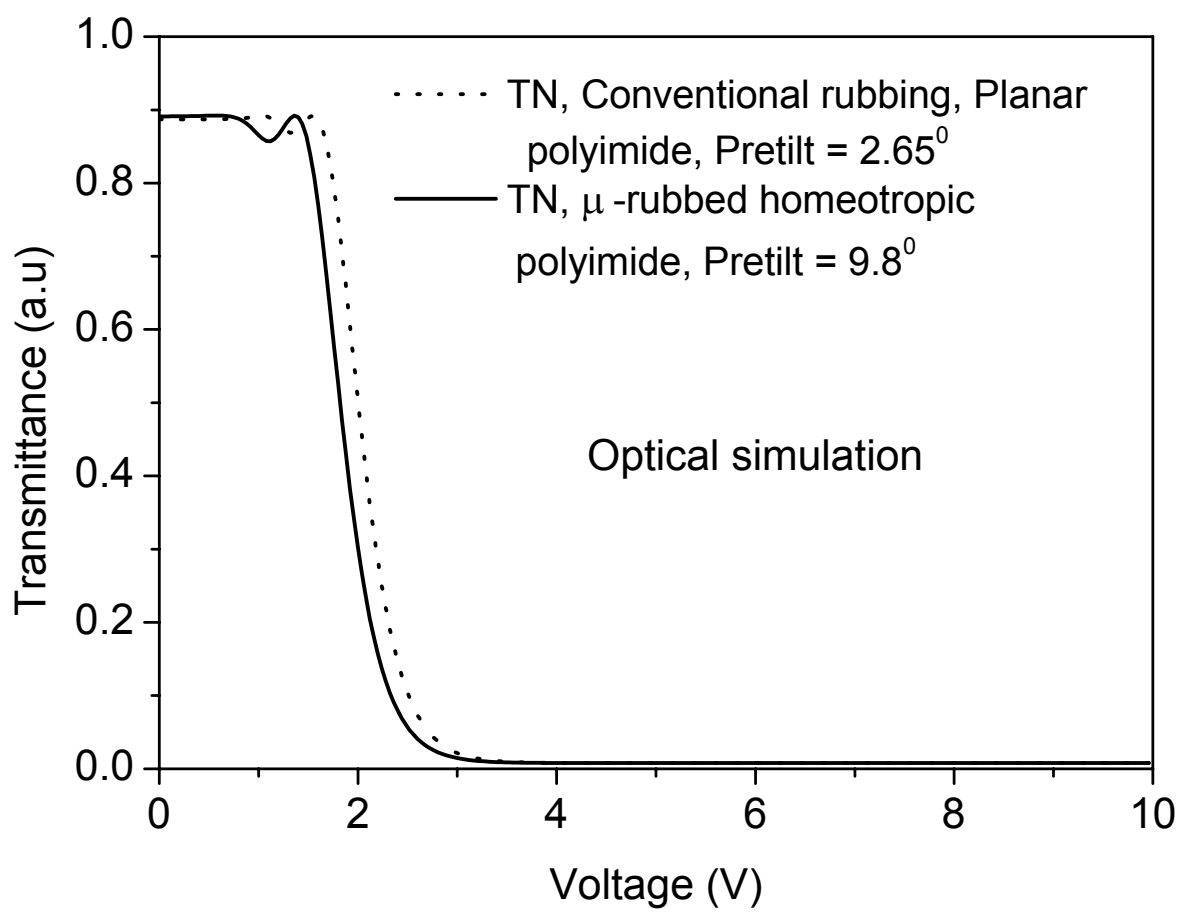

Figure 3.13: Comparison of switching behavior from optical simulation between two TN areas with different surface pretilt angle. 


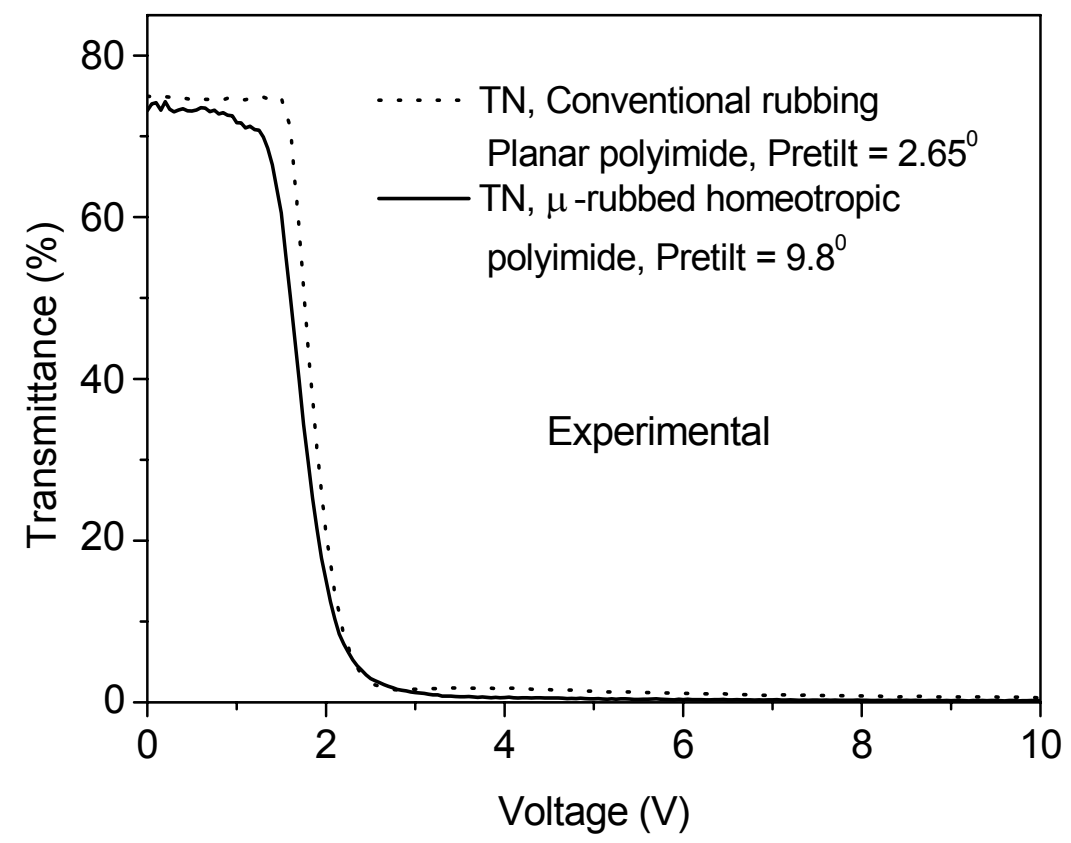

Figure 3.14: Comparison of switching behavior from experiment between the conventionally rubbed polyimide and the $\mu$-rubbed homeotropic polyimide.

\subsubsection{Alignment mechanism}

The $\mu$-rubbing of homeotropic polyimide enforces planar alignment with a high surface pretilt angle. The following physical mechanism is proposed for the modification of the alignment surface from homeotropic (before $\mu$-rubbing) to a planar alignment with large pretilt (after $\mu$-rubbing). Polyimides that are known to induce homeotropic anchoring are either doped with long chain aliphatic or are functionalized with long chain aliphatic molecules. When aliphatic chains pack on a substrate parallel to the substrate normal (highly aligned, compact assembly of aliphatic chains), they are known to induce homeotropic alignment of liquid crystal molecules coming in contact with them. ${ }^{19}$ It can be conjectured that the $\mu$-rubbing process unidirectionally aligns the aliphatic chains at a specific angle as schematically shown in Fig. 3.15. This mechanism leads to a dramatic change in the anchoring direction for liquid crystal molecules interacting with the aliphatic rich surface. 


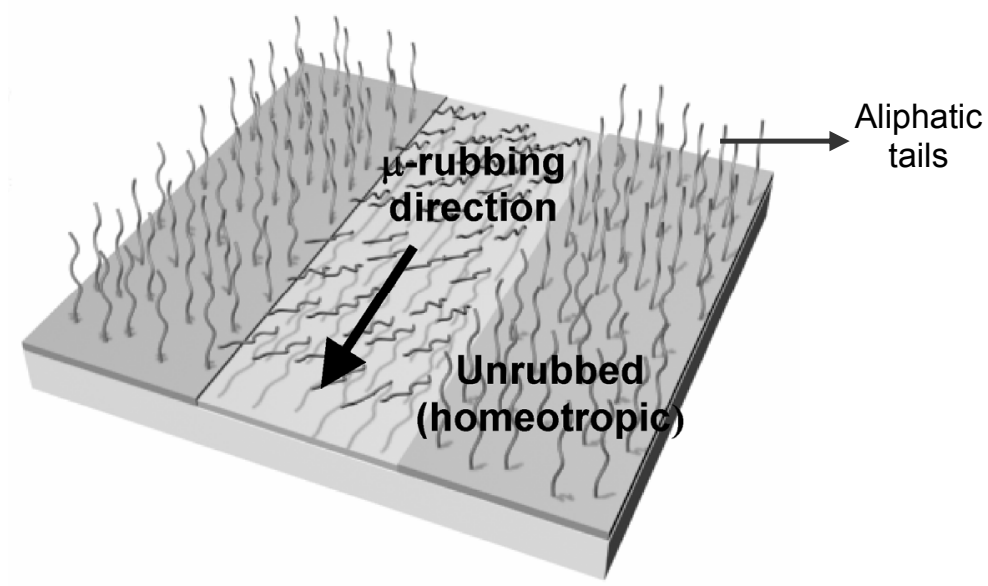

Figure 3.15: A schematic illustration of a surface rich in aliphatic moieties and its deformation during the $\mu$-rubbing process.

On a macroscopic level the state during which there is contact of the polymer film and the metallic sphere can be considered as being intermediate between elastic and plastic contact states. If the contact state were elastic, the polymer film would fully recover from the surface deformation and/or the reorientation of polymer chains after passing the metallic sphere. If so the polymer surface could not act as a LC alignment layer. For a good alignment a plastic deformation state must be induced in the polymer surface. ${ }^{20}$ The plastic deformation increases with applied load and it leads to an increase in surface roughness.

The $\mu$-rubbing of homeotropic polyimide was described in this chapter and the experimental results were compared with the optical simulations. It was shown that the $\mu$-rubbing results in the planar alignment of LC with high surface pretilt angle. The $\mu$ rubbing also opens the possibility to vary the pretilt by changing the applied load. The $\mu$ rubbing on homeotropic polyimide potentially useful to fabricate a stable multidomain structure, for instance TN-LCDs which is expected to improve the viewing angle. This topic is dealt with more extensively in the next chapter. 


\subsection{Conclusions}

In this chapter patterning of homeotropic alignment layers using a $\mu$-rubbing technique is described. The variation of pretilt angle with applied load is explained and an inverse relation is found between applied load and pretilt angle. The $\mu$-rubbing creates multiconfigurations consisting of twisted nematic, splay and homeotropic states. It demonstrated that when polyimide that originally induces homeotropic alignment is $\mu$ rubbed, the $\mu$-rubbing induces a planar alignment with a high pretilt. The electro-optical measurements are consistent with this observation, showing a near thresholdless switching behavior. The optical simulation results show good agreement with the experimental observations.

\subsection{References}

[1] S. Furumi, K. Ichimura, Adv. Funct. Mater. 14, 247 (2004).

[2] J. Cognard, Mol. Cryst. Liq. Cryst., Suppl. Ser. 1, 1 (1982).

[3] A. J. Pidduck, G. P. Bryan-Brown, S. Haslam, R. Bannister, I. Kelly, T. J. McMaster, L. Boogaard, J. Vac. Sci. Technol. A, 14, 1723 (1996).

[4] S. H. Paek, C. J. Durning, K. W. Lee, A. Lien, J. Appl. Phys., 83, 1270 (1998).

[5] J. Lee, D. Seo, Liq. Cryst. 27, 711 (2000).

[6] J. C. Jung, K. H. Lee, B. S. Sohn, S. W. Lee, M. Ree, Macromol. Symp. 164, 227 (2001).

[7] D. Seo, S. Kobayashi, Liq. Cryst. 27, 883, (2000).

[8] W. M. Gibbons, P. J. Shannon, S. T. Sun, B. J. Swetlin, Nature 351, 49 (1991).

[9] M. Schade, K. Schmitt, V. Kozenkov, V. Chigrinov, Jpn. J. Appl. Phys. 31, 2155 (1992).

[10] M. Hasegawa, Y. Taira, J. Photopolym. Sci. Technol. 8, 241 (1995).

[11] Chen, J., Bos, P. J., Johnson, D. L., Bryant, D. R., Li, J., Jamal, S. H., Kelly, J. R., J. Appl. Phys. 80, 1985, (1996).

[12] G. Baur, V. Witter, D. W. Berreman, Phys. Lett. A56, 142 (1976). 
[13] B. L. V. Horn and H. H.Winter, Appl. Opti. 40, 2089 (2001).

[14] 2dimMOS, www.autronic-melchers.com

[15] M. E. Becker, H. Wohler, M. Kamm and J. Kreis, Proceedings of SID 96, 596 (1996).

[16] L. M. Blinov, V. G. Chigrinov, Electro-optic effects in liquid crystal materials, Springer-Venlag, Chapter 4, New York, (1996).

[17] P. Yeh, C. Gu, Optics of liquid crystal display, John Wiley \& Sons, Inc. (1999).

[18] J. Nehring, A. R. Kmetz, T. J. Scheffer, J. Appl. Phys., 47, 850 (1976).

[19] G. Porte, J. De Physique, 37, 1245 (1976).

[20] M. Honma, T. Nose, Jpn. J. Appl. Phys. 42, 6992 (2003). 


\section{Chapter 4}

\section{Multidomain Twisted Nematic Liquid Crystal Display by $\mu$-Rubbing*}

\subsection{Introduction}

In the liquid crystal display (LCD) industry much attention is focused on improving the viewing angle of displays. The quality of the viewing angle has become even more important with the recent launch of LCDs into the large area display market, e.g. for use as television screens. The viewing angle problem in conventional twisted nematic LCDs arises from the fact that the midplane liquid crystal director angle (optic axis) is uniformly oriented in one direction creating a highly anisotropic viewing cone. Due to the inherent birefringence of liquid crystal materials, different viewing directions give varying degrees of birefringence, resulting in an optical transmission behavior strongly dependent on the viewing direction. ${ }^{1}$ Furthermore, the electro-optic switching characteristics of uniformly aligned TN displays are strongly dependent on the viewing angle, a serious problem for stable gray scale performance, which is a prerequisite for full color, high resolution displays. ${ }^{2}$

There are many approaches to overcome the poor viewing angle performance in LCDs, including compensation films integrated on the outside of the display cell between the polarizer films, ${ }^{3-8}$ modifications inside the display cell involving multidomain alignment, ${ }^{9-17}$ vertical alignment ${ }^{18-20}$ and alternative modes of operation such as in-plane switching (IPS). ${ }^{21-23}$ Multidomain alignment requires a number of lithographic steps, ${ }^{9-12}$ which is unattractive for manufacturing; therefore, researchers have been focused on

*This chapter is partly reproduced from: S. Varghese, G. P. Crawford, C. W. M. Bastiaansen, D. K. G. de Boer, D. J. Broer, Appl. Phys. Lett. 85, 230 (2004), J. Appl. Phys. 97, 053101 (2005) and Mol. Cryst. Liq. Cryst. (in press), (2005). 
accomplishing the same goal using patterned photo alignment, ${ }^{24}$ oblique evaporation of silicon oxide $^{25,26}$ and writing with an atomic force microscopy (AFM) tip. ${ }^{27,28}$

In Chapter 2, a new $\mu$-rubbing technique was introduced to create alignment patterns on polyimide alignment layers. It was shown a planar alignment is obtained with planar polyimides and the pretilt of the LCs are low $\left(<1^{\circ}\right)$. As a consequence, the formation of stable, multidomain TN displays was impossible and new routes were explored to enhance the pretilt. In Chapter 3, $\mu$-rubbing of a homeotropic polyimide was investigated and it was found that high pretilt $\left(9^{\circ}-16^{\circ}\right)$ could be obtained. This higher pretilt opens the possibility for an increase in the stability of multidomain pixels, this issue is re-addressed in this chapter.

\subsection{Experimental}

\subsubsection{Materials}

The polyimide precursor for the orientation layer AL-75114 was purchased from JSR Electronics. Indium tin oxide (ITO) coated glass $(25 \mathrm{~mm} \times 25 \mathrm{~mm})$ was obtained from Merck. The ITO coated glass substrate was thoroughly washed with soap solution and deionised water followed by ethanol and dried. The polyimide precursor was spin coated ( $5 \mathrm{~s}$ at $1000 \mathrm{rpm}$ followed by $40 \mathrm{~s}$ at $5000 \mathrm{rpm}$ ) on the ITO side of the glass substrate. It is then preheated to $100{ }^{\circ} \mathrm{C}$ for $10 \mathrm{~min}$ and imidized at $180{ }^{\circ} \mathrm{C}$ under vacuum for 90 minutes resulting in a transparent film with a thickness of $\sim 100 \mathrm{~nm}$. The liquid crystal E7, a mixture consisting of $50.6 \%$ 4'-pentylcyanobiphenyl (5CB), $25.2 \%$ 4'heptylcyanobiphenyl (7CB), $17.8 \%$ 4'-octyloxycyanobiphenyl (8OCB), and $6.4 \%$ 4'pentylcyanoterphenyl (5CT), was obtained from Merck Ltd. (Darmstadt, Germany).

\subsubsection{The $\mu$-rubbing process}

The polyimide alignment layer induces homeotropic anchoring of the liquid crystal in the unrubbed state was used. The metallic sphere is in direct contact with the alignment layer as it travels across the alignment layer (substrate) creating micrometer sized rubbed lines 
at a velocity of $10 \mathrm{~mm} / \mathrm{min}$ under specified load $(150 \mathrm{~g})$ at room temperature. In this $\mu$ rubbing technique the polyimide surface is rubbed in such a way that neighboring alignment regions are rubbed in opposite directions to create multi domain pixels (see Fig. 4.1a).

\subsubsection{Cell construction}

Liquid crystal cells were constructed with two $\mu$-rubbed substrates placed orthogonal to each other. The cells were secured with UV curable glue (Norland UV sealant 91) along the edges. The cell gap was controlled with $5 \mu \mathrm{m}$ spacers. The cells were filled with the liquid crystal E7 by capillary action at $80{ }^{\circ} \mathrm{C}$, which is $\sim 20^{\circ} \mathrm{C}$ above the nematicisotropic transition temperature of the liquid crystal.

\subsubsection{Characterization}

Surface morphology of the $\mu$-rubbed polyimide layer was investigated by atomic force microscopy (AFM), Nanoscope Dimension 5000 with a nanoscope III controller (Digital instruments, Santa Barbara, CA) with a scan rate of $1 \mathrm{~Hz}$. Cell gap measurements were carried out using Shimadzu UV-3102 PC UV-Vis-NIR scanning spectrophotometer. Electro-optical measurements were performed on a DMS 703 display measuring system (Autronic-Melchers Gmbh). An AC square wave was used to drive the display cells (1 $\mathrm{kHz}$ ). The alignment of liquid crystals was studied using polarized light microscopy, Zeiss LM Axioplan. The pretilt angle was measured directly using an Autronic, TBA 107 instrument that uses the crystal rotation method $^{29}$ (see Chapter 2). Viewing angle characteristics were measured using an Eldim conoscope up to an azimuthal angle of $60^{\circ}$ at various voltages using a waveform generator. 


\subsection{Results and discussion}

\subsubsection{Surface characterization}

Figure 4.1a shows the $\mu$-rubbing set up used to create multidomain pixels in which the rubbing directions of the neighboring lines are in opposite direction and the lines are touching each other.

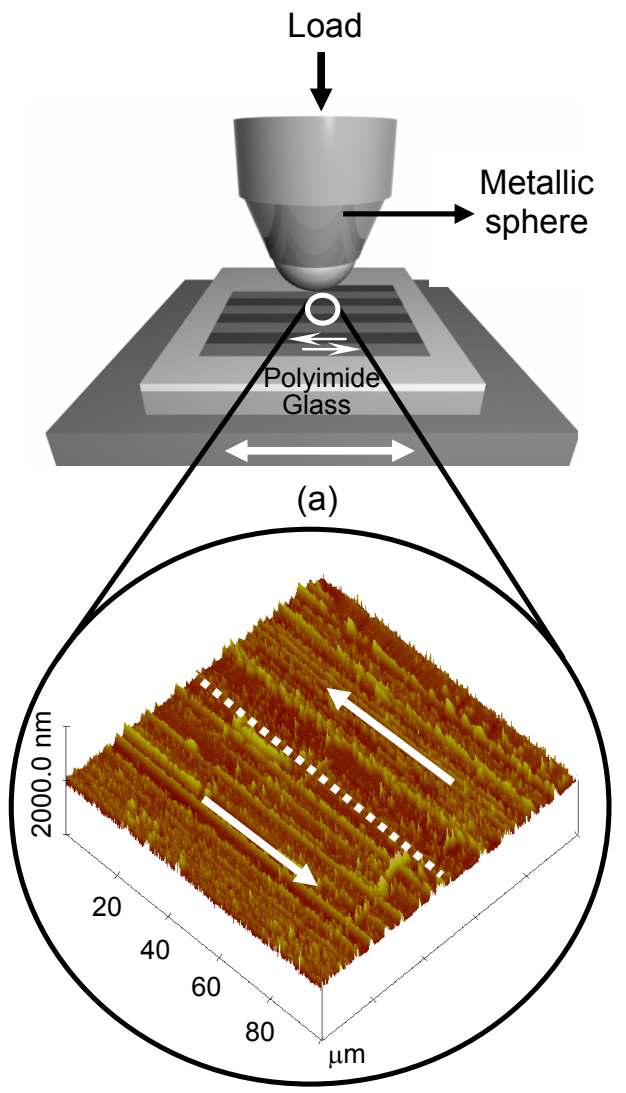

(b)

Figure 4.1: (a) Illustration of experimental setup for the $\mu$-rubbing process, the arrows show the direction of $\mu$-rubbing. (b) Atomic force microscopy image of the $\mu$-rubbed surface where homeotropic polyimide having two lines with opposite rubbing direction are adjacent to each other. 
Figure $4.1 \mathrm{~b}$ shows the AFM image of the $\mu$-rubbed surface. The single line width is found to be $\sim 47 \mu \mathrm{m}$ with an average surface roughness of $40 \pm 2 \mathrm{~nm}$ as determined by AFM. As can be observed from the AFM measurements in Fig. 4.1b, $\mu$-rubbing does generate grooves on homeotropic polyimide with a width of several micrometers. As mentioned in Chapter 3 , it is hypothesized that the $\mu$-rubbing unidirectionally aligns the aliphatic chains of the homeotropic polyimide at a specific angle. This leads to a dramatic change in the anchoring direction of liquid crystal molecules interacting with the surface. Figure 4.2 shows a schematic illustration of the alignment of LC molecules on the $\mu$ rubbed surface. The rubbing directions of adjacent lines are opposite to each other, which leads to an opposite surface pretilt angle.

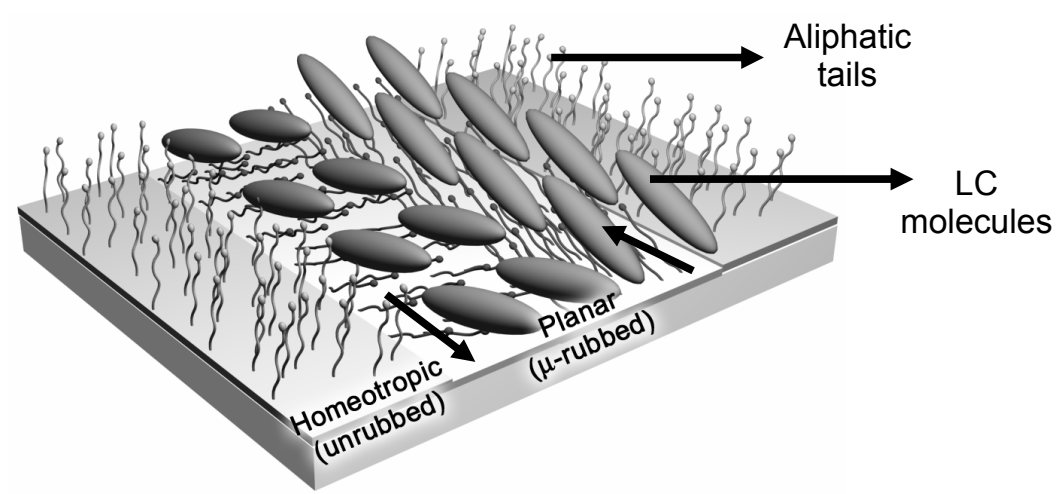

Figure 4.2: A schematic illustration of a surface rich in aliphatic moieties and its deformation during the $\mu$-rubbing process. The alignment of LCs on this $\mu$-rubbed surface is also shown.

Figure 4.3a shows the rubbing directions on the top and bottom substrates. The orthogonal alignment of the liquid crystal molecules anchored to the surface and the tilt angle impose a twist, a direction (handedness) and the average tilt over the cross section of the layer. Figure $4.3 \mathrm{~b}$ shows the midplane twist and tilt directions of the various domains in the center of the cell. A 3D rendition of a multidomain pixel is depicted in Fig. $4.3 \mathrm{c}$. Due to the difference in rubbing direction in this multidomain configuration, there are two left-handed and two right-handed sub-pixels. The midplane directors are oriented at an angle of $45^{\circ}$ with the $\mu$-rubbing directions (Fig. $4.3 \mathrm{~b}$ ). 

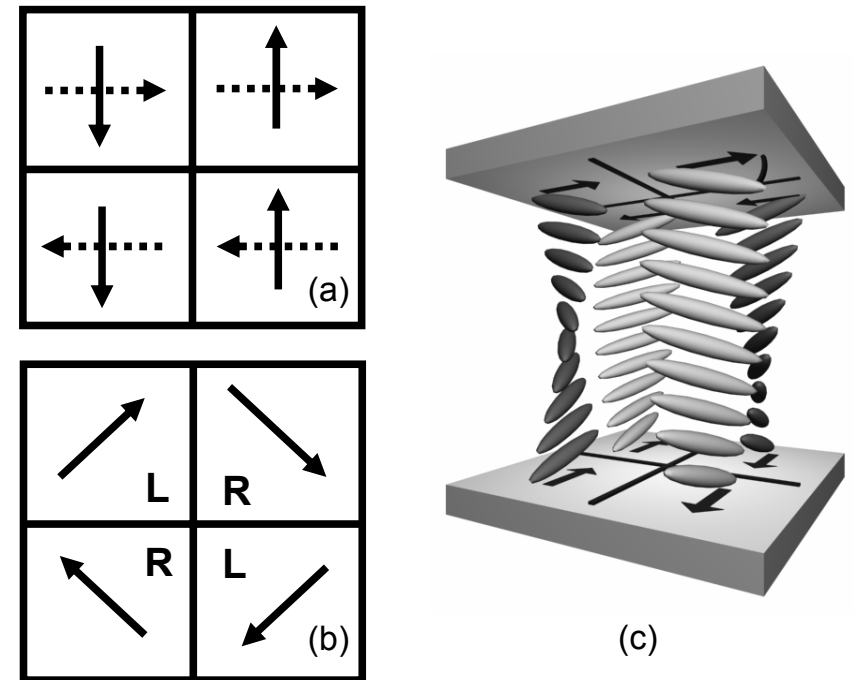

(c)

Figure 4.3: Orientation of liquid crystal directors in four different domains. (a) Solid and dotted arrows indicate the rubbing direction of top and bottom substrates. (b) The direction of the midplane directors in the four-domains, $L$ and $R$ indicate left and right twist of the liquid crystal. (c) Four-domains schematically showing the opposite twist sense in each sub-pixel.

\subsubsection{Stability of four-domain structure}

In Fig. 4.4 optical micrographs of a four-domain TN-LCD at different voltages as observed between crossed polarizers are shown. These micrographs illustrate a stable multidomain configuration is obtained at high voltages $(>1 \mathrm{~V})$. Well aligned, square shaped areas are separated by black disclination lines marking the transitions between the different liquid crystal alignments in the sub-pixels. These optical microscopy photographs where taken after applying a high voltage $(5 \mathrm{~V})$ and slowly decreasing it so it completely locks-in (stabilizes) the motion of disclination lines. The process during increasing and decreasing voltage can be followed in more detail in Fig. 4.7. Below $1 \mathrm{~V}$ the motion of disclination lines is observed and the disclination lines no longer correspond to the position of the sub-pixels, which indicates the multidomain configuration is unstable below $1 \mathrm{~V}$. The twisted states in the samples are determined by the pretilt angle. A large surface pretilt angle is required to compensate for the energy 
cost of the generation of twist disclinations. In order to ascertain the stability of the fourdomain samples in zero fields, one needs to compare the free energy cost associated with forming disclination lines and the wrong handed sub-pixel orientations that cost splay energy. In the polarizing microscopy photographs, stable disclination lines are visible above $1 \mathrm{~V}$ when coming down from high voltage.
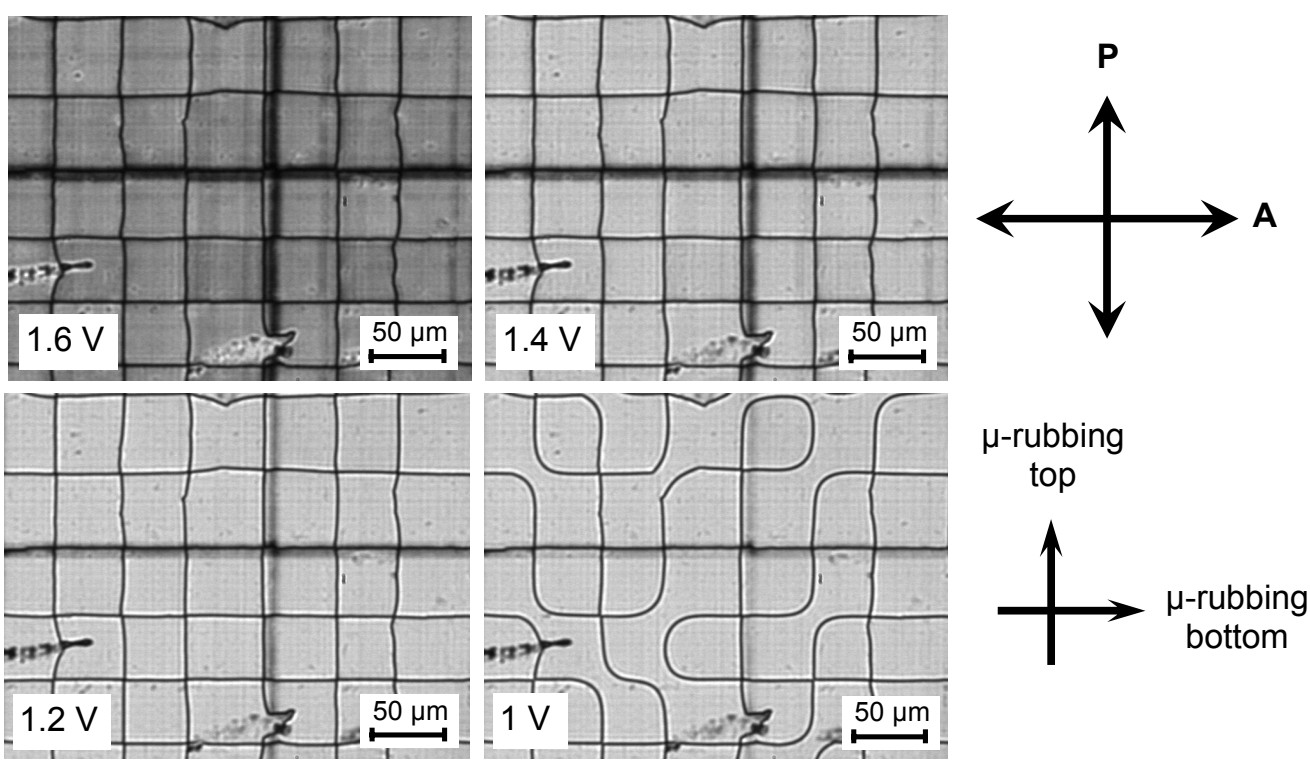

Figure 4.4: Optical microscopy pictures showing the stability of four-domain TN-LCDs at different voltages; respective polarization and rubbing directions are also shown. The sample is first switched to $5 \mathrm{~V}$ and subsequently the voltage is decreased.

In Chapter 2, a model was used to estimate the minimum pretilt required for stable multidomain formation. Based on this model it was estimated how the required pretilt $\theta_{p}$ depends on the sub-pixel dimensions $L \times L$, the cell gap $d$, and the known elastic properties of nematic liquid crystals. The stability condition can be expressed by the following equation: $\theta_{p}{ }^{2} \geq \pi d / L$. Using $L \sim 50 \mu \mathrm{m}$ and $d \sim 5 \mu \mathrm{m}$, the predicted minimum pretilt angle, $\theta_{p, \min }$, to stabilize the structure is $\theta_{p, \min } \sim 30^{\circ}$. 25 
In this case, the $\mu$-rubbed homeotropic alignment layers have a pretilt of $\sim 10^{\circ}$ and consequently, a four-domain stable structure is not observed at low voltages $(0-1 \mathrm{~V})$. Although this model is simple, it is consistent with the observations and the work presented in the literature. ${ }^{25,26}$ Therefore, in the zero voltage and low voltage states where the disclination lines disappear or are unstable, the four-domain sample essentially transforms into a two-domain reverse tilt sample. It is possible to stabilize the fourdomain structure using a voltage initialization process and driving the sample with bilevel voltages. This scheme can completely lock-in the motion of disclination lines. ${ }^{26}$ This phenomena is demonstrated here by reducing the voltage after domains were formed at high voltage (see Fig. 4.7). The larger dark lines in Fig. 4.4 at $2 \mathrm{~V}$ are due to a small lateral gap between two $\mu$-rubbed lines (splay configuration with one surface having homeotropic alignment and another surface having parallel alignment). The occurrence of this is due to the spatial resolution of the $\mu$-rubbing apparatus.

Figure 4.5 shows the enlarged optical micrograph of the stable four-domain structures (at $2 \mathrm{~V}$ ) in which the midplane directors are oriented in the four quadrants as indicated by the arrows. The two different bias tilt directions on each substrate define the appropriate sense of each of the twisted sub-pixels.
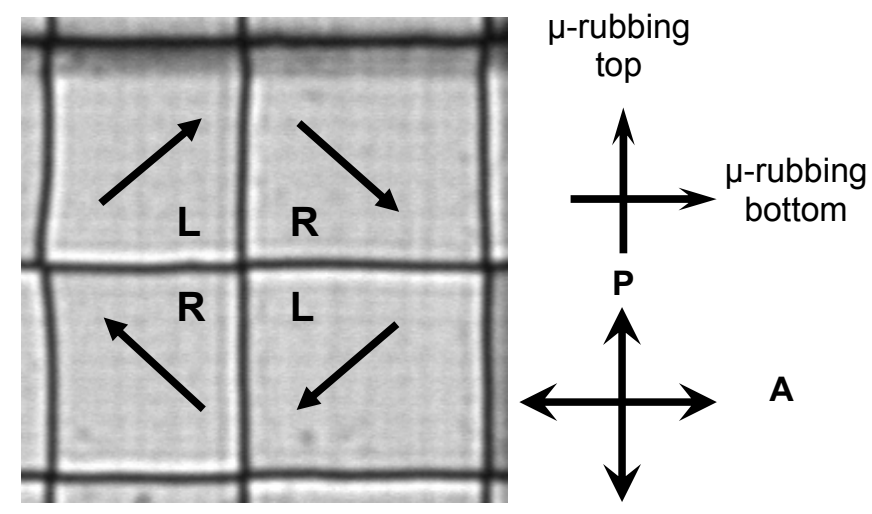

Figure 4.5: Optical microscopy image of the four-domain structure which is stable above $1 \mathrm{~V}$. The direction of midplane directors are indicated by arrows, which are oriented in four corners of the sub-pixels. 
Figure 4.6 shows the intensity profiles obtained by line scanning across the disclination lines of the optical microscopy images at different voltages $(5 \mathrm{~V} \rightarrow 1 \mathrm{~V})$. Figure 4.6 also shows that intensity of the disclination lines decreases with increasing voltage and at $5 \mathrm{~V}$ (dark state) they are no longer visible. Of course, the disappearance of the disclination lines is to be expected because in each quadrant the liquid crystal molecules align along the electrical field lines, removing transitions in molecular orientation in the dark state of the display. The defects (disclination lines) at low voltages reduce the contrast ratio, but their contribution is small because the display is in the bright state.
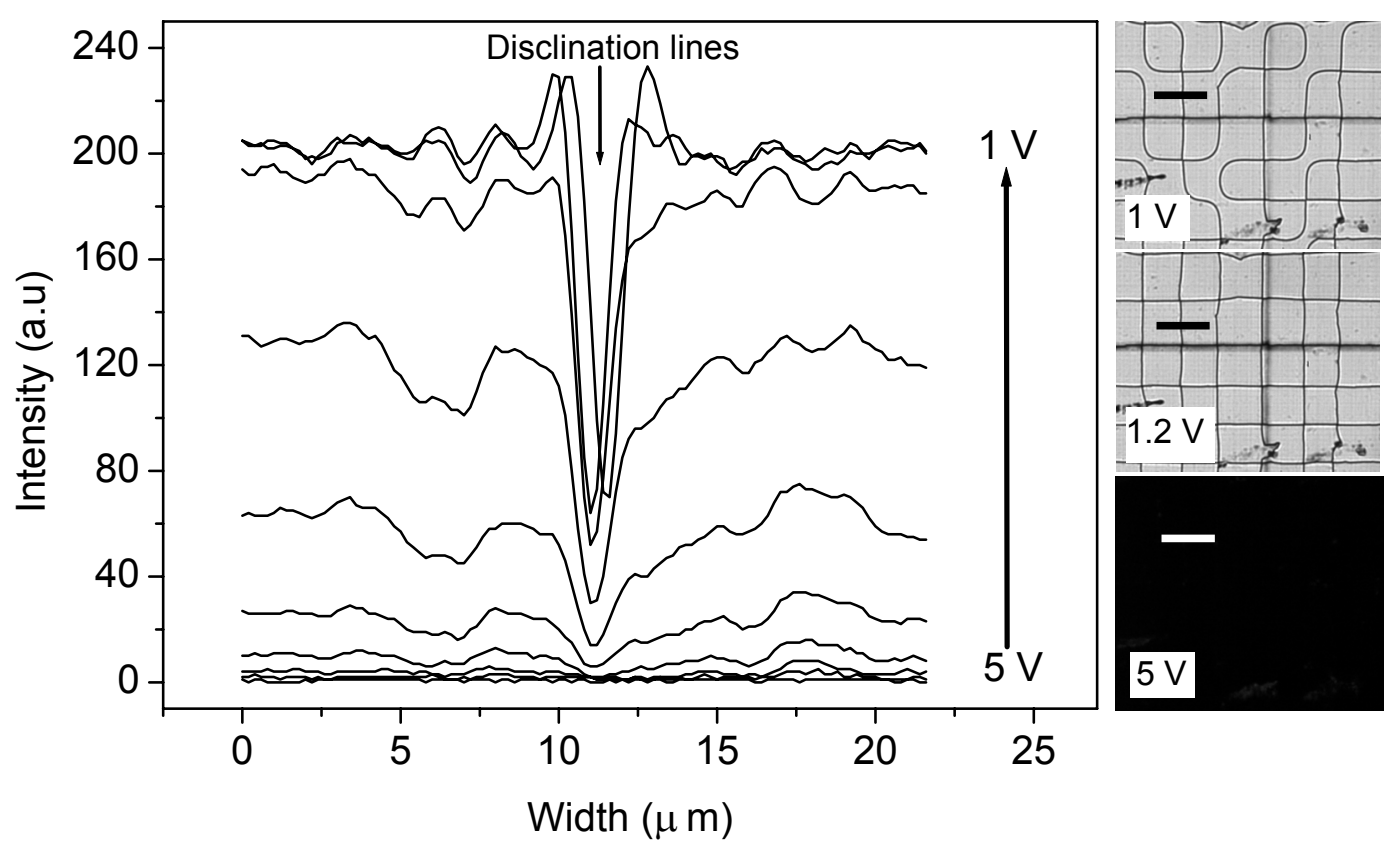

Figure 4.6: Intensity profile obtained from the line scanning of optical micrographs of the fourdomain cell at different voltages ( 1 to $5 \mathrm{~V}$ at an interval of $0.5 \mathrm{~V}$ ). The dark line in the optical micrographs (top left) shows the area of line scanning.

\subsubsection{Viewing angle characteristics}

To study the performance of the multidomain structure in a real display, the viewing angle characteristics were measured. Figure 4.7 shows the viewing angle characteristics of the four-domain TN display. 


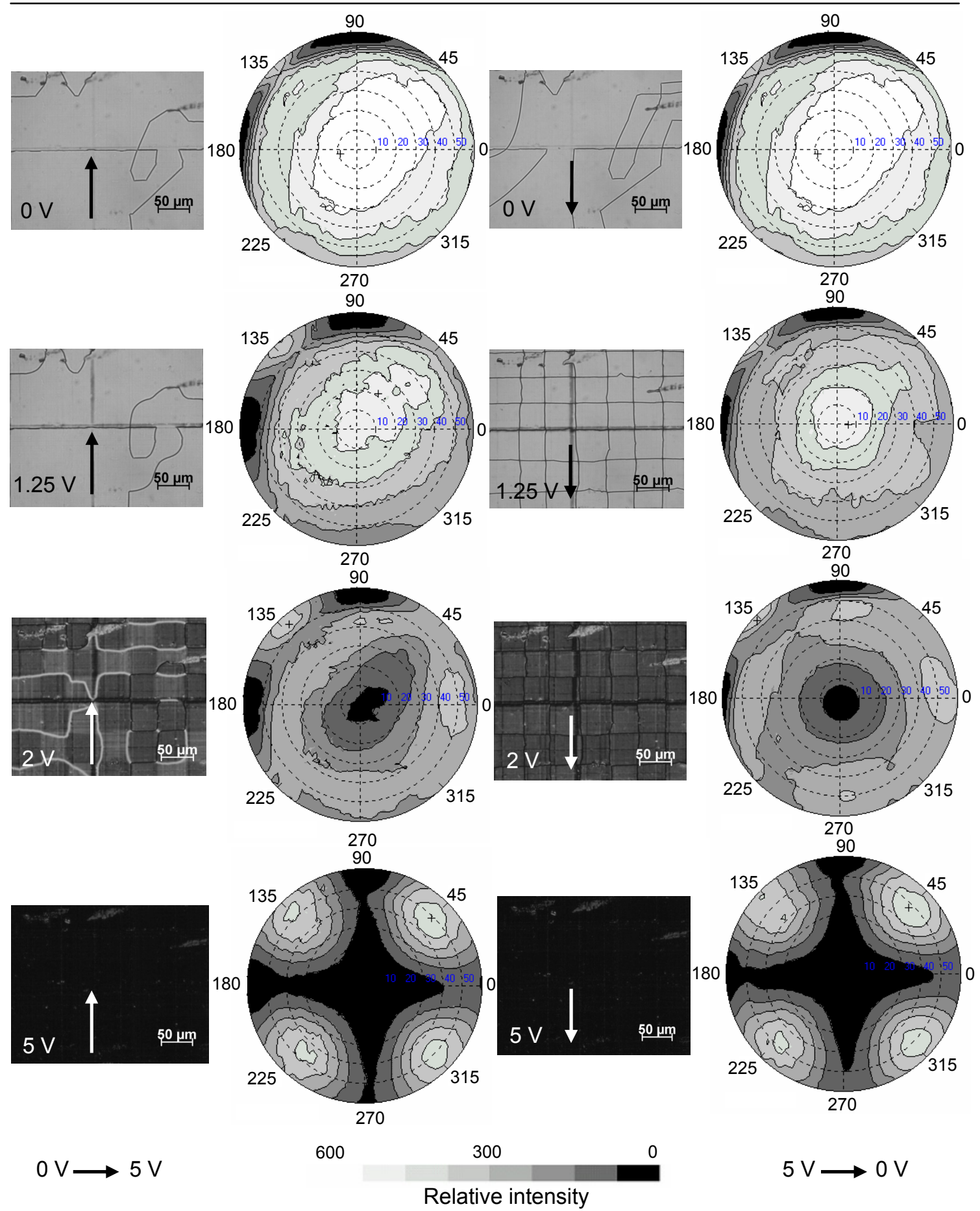

Figure 4.7: Optical microscopy images of a four-domain twisted nematic cell created by the $\mu$ rubbing process viewed between crossed polarizers and subject to an applied voltage, left side, 0 $5 \mathrm{~V}$ and right side 5-0 $\mathrm{V}$. The isointensity plots as a function of viewing angle of the four-domain TN cell at the same voltage is also shown. 
The plots represents isointensity lines with respect to different viewing angles upto $60^{\circ}$. Figure 4.7 (left half) represents the polarizing optical microscopy photographs of a four-domain TN cell and its corresponding viewing angle characteristics measured with an Eldim conoscope as a function of applied voltage $0 \mathrm{~V} \rightarrow 5 \mathrm{~V}$ (increasing voltage) and Fig. 4.7 (right half) represents the same for decreasing voltage $(5 \mathrm{~V} \rightarrow 0 \mathrm{~V})$. The two different bias tilt directions on each substrate define the appropriate sense of the four sub-twisted pixel configurations.

On a macroscopic scale, the optical anisotropies of the differently aligned midplane tilt angles of the four sub-helices compensate each other as depicted schematically in Fig. 4.3b and Fig. 4.3c. The angular dependence of a four-domain display is significantly reduced as compared to conventional single domain TN LCDs. ${ }^{26}$ When the sample is switched from 0 to $5 \mathrm{~V}$ in small increments, the formation of unstable disclination lines at $2 \mathrm{~V}$ is observed and it transforms into a perfectly dark state at $5 \mathrm{~V}$ (homeotropic). The four-domain structure becomes stabilized for voltages $>2 \mathrm{~V}$. When slowly reducing the voltage from 5 to $0 \mathrm{~V}$, clear disclination lines can be observed indicating a stable four-domain configuration over a large voltage range. In the viewing angle measurements of Fig. 4.7 one can observe, upon lowering the voltage to $1.25 \mathrm{~V}$, the symmetric nature of the isointensity curves, inherent to the multidomain twisted nematic configuration. $^{24,25}$

\subsubsection{Contrast ratio and color properties}

The iso-contrast measurements of a single domain and a four-domain cell are shown in Fig. 4.8. Comparing these images one can directly understand the improvement in contrast uniformity and symmetry, especially at higher viewing angles. Also, the gray scale inversion is improved for the four-domain structure. In the direction perpendicular or parallel to one of the polarizers, the contrast ratio is $40: 1$ at angles $> \pm 50^{\circ}$. At $45^{\circ}$ to the polarizers a contrast ratio of $10: 1$ is possible up to $\pm 30^{\circ}$. The contrast ratio at $1.25 \mathrm{~V}$ is important because the four-domain is stable in that voltage range and it is slightly less than in the zero field state. 


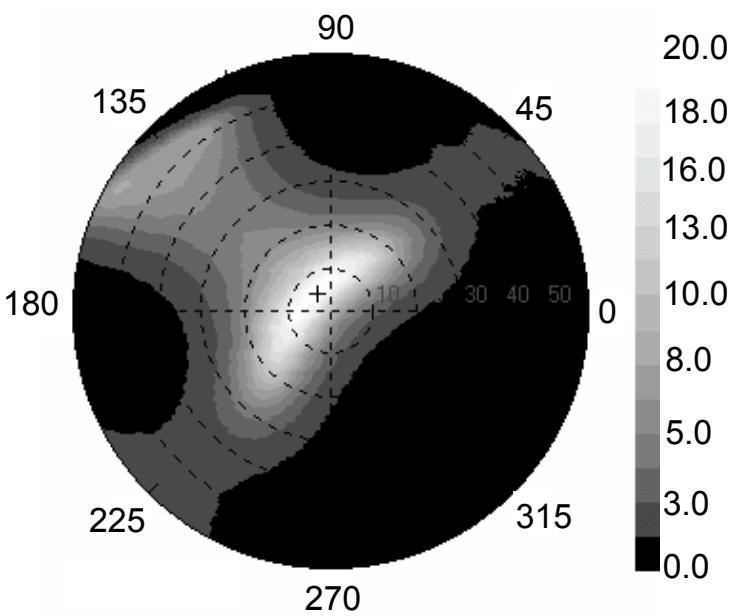

(a)

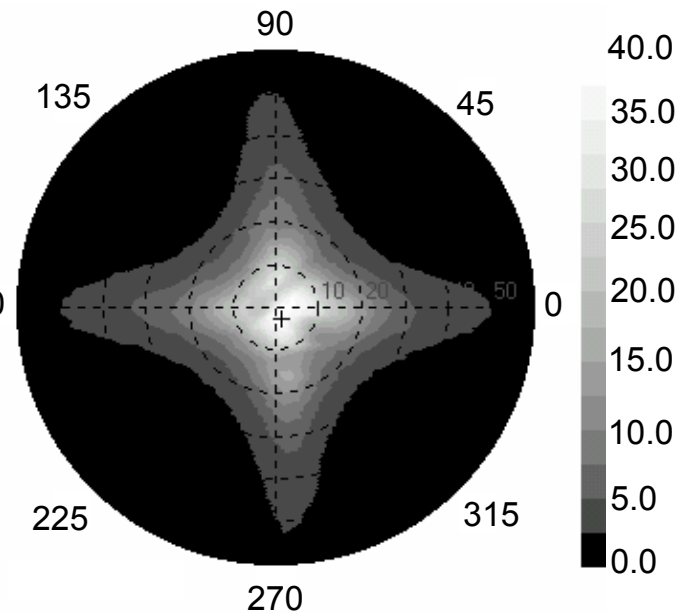

(b)

Figure 4.8: The iso-contrast measurement of the single domain (a) and four-domain liquid crystal cell (b).

Figure 4.9 shows the color shift of the four-domain cell in the fully bright state at $60^{\circ}$ and $80^{\circ}$ viewing angles. The black dots represent the points of measurement at different angles. Due to the self-compensation effects in the four-domain cell, the dependency of white color chromaticity on azimuthal viewing directions is substantially less at $60^{\circ}$ if compared to $80^{\circ}$. All points are concentrated near yellow and white region $(x=0.4$ and $y=0.4)$ indicating a narrow distribution of color. Since the midplane liquid crystal directors are oriented in four different directions, at $45^{\circ}$ with respect to the polarization axes of the polarizers, the azimuthal angle dependency of phase retardation is minimized.

Figure 4.10 shows the transmission voltage characteristics of the four-domain cell when a field is applied from $0 \mathrm{~V} \rightarrow 5 \mathrm{~V}$ and $5 \mathrm{~V} \rightarrow 0 \mathrm{~V}$. The slight hysteresis in transmittance near the threshold area when coming down from higher voltages is due to the presence of disclination lines. 

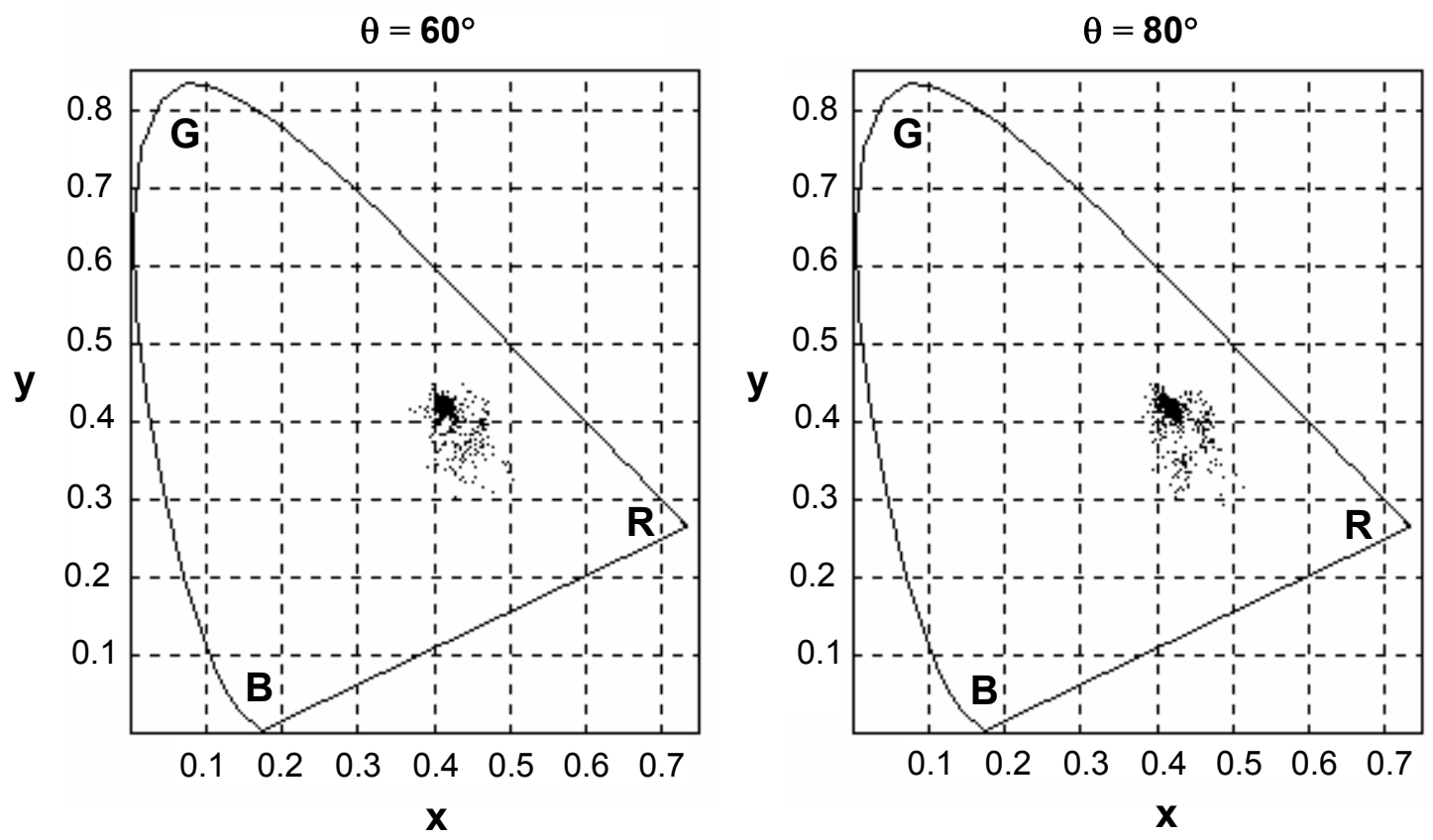

Figure 4.9: Dependence of white color chromaticity at two polar angles, $60^{\circ}$ and $80^{\circ}$ on azimuthal viewing angle.

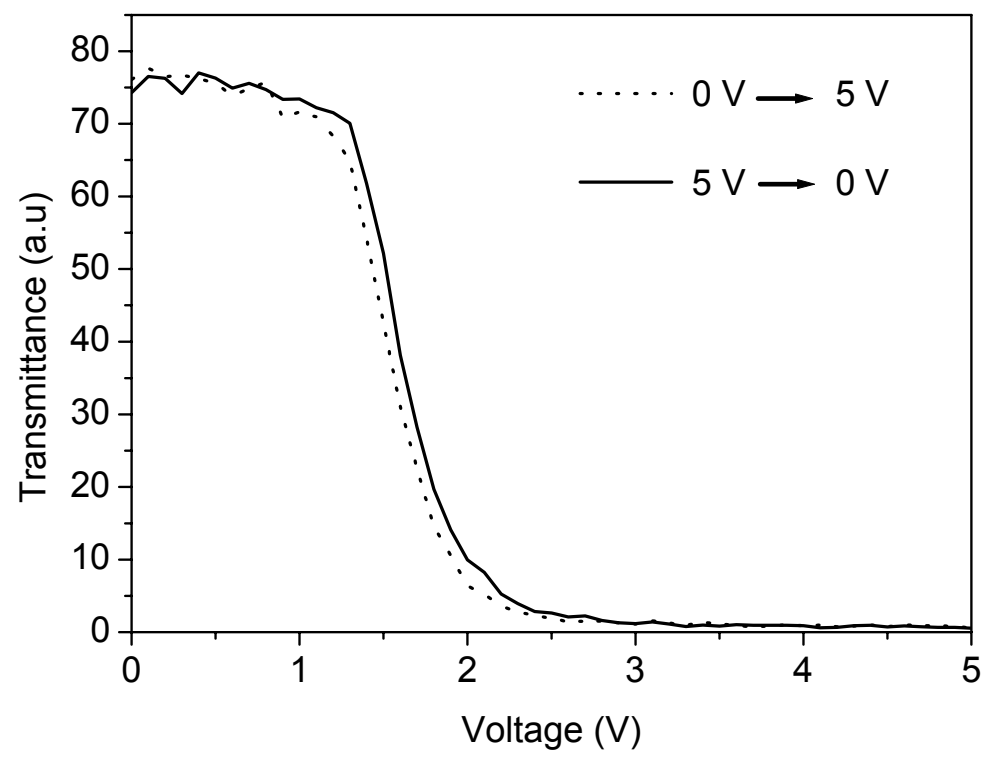

Figure 4.10: Transmission-voltage curve of the four-domain cell from $0 \mathrm{~V} \rightarrow 5 \mathrm{~V}$ and $5 \mathrm{~V} \rightarrow 0 \mathrm{~V}$. 
The transmission of the four-domain TN-LCD was measured with different wavelengths of light as shown in Fig. 4.11. It is clear from the graph that the fourdomain TN-LCD is only slightly sensitive to wavelength variation, suitable for a full color display.

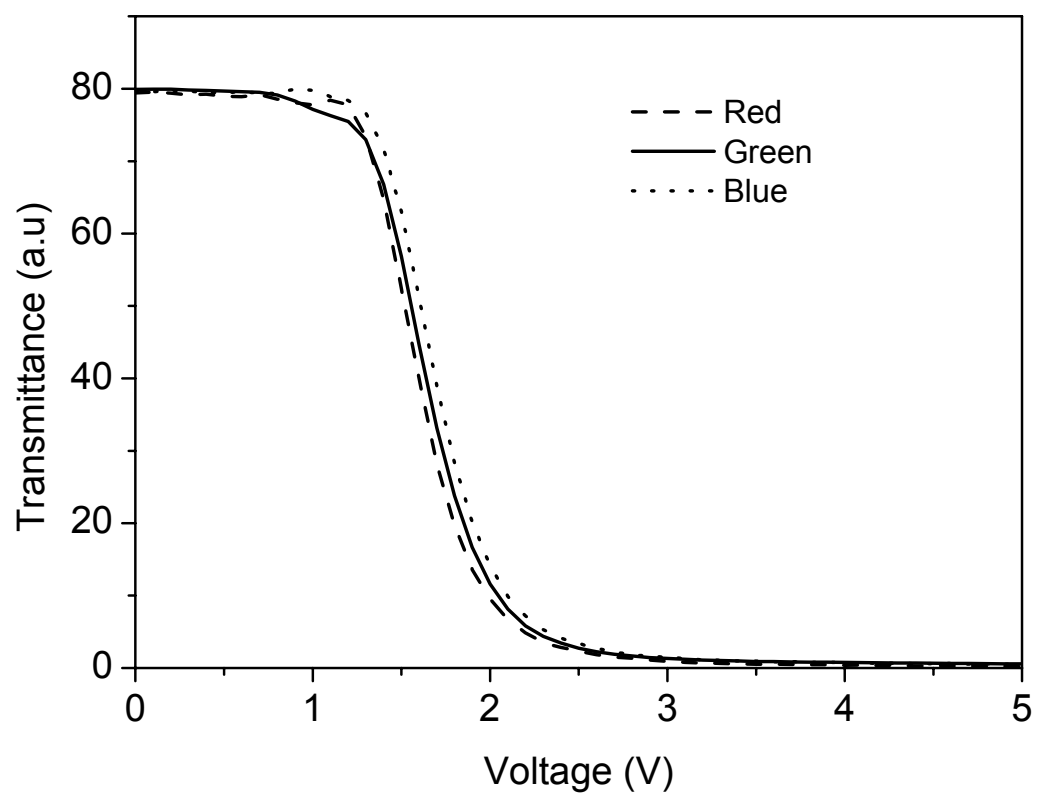

Figure 4.11: The transmission at normal incidence as a function of voltage of the four-domain TN cell for different wavelength of light.

\subsubsection{Heat aging}

Because the controlled four-domain formation in these samples is supposed to be the result of a re-alignment of packed aliphatic moieties at the polyimide surface, there is a possibility of relaxation of these units and a corresponding loss of orientation and favorable electro-optical properties. Therefore, a thermal aging study was performed on the sample by heating it to $100{ }^{\circ} \mathrm{C}$ and keeping isothermally for a duration of 15 minutes and then cooling it to room temperature at $20{ }^{\circ} \mathrm{C}$. The sample was then allowed to stabilize at $20{ }^{\circ} \mathrm{C}$ for 15 minutes before taking the transmission-voltage data. This heating protocol was repeated five times and the transmission-voltage curves after each step are shown in Fig. 4.12. The transmission-voltage curve in Fig. 4.12 remains 
relatively unchanged with only a minor shift of $\Delta \mathrm{V} \sim 0.1 \mathrm{~V}$ (at $50 \%$ transmission). This brought to the conclusion that the alignment imposed by $\mu$-rubbing a homeotropic polyimide is sufficiently stable and robust to serve in applications such as high-end displays.

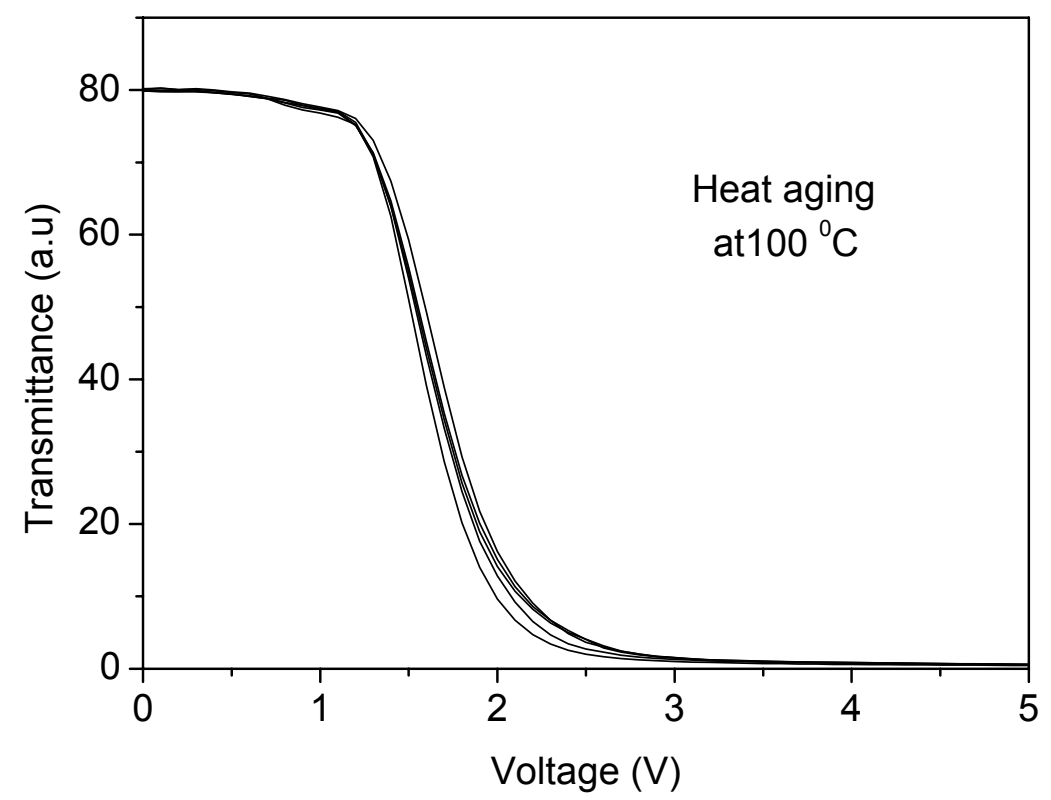

Figure 4.12: Transmission-voltage curve of the four-domain cell after heat aging at $100{ }^{\circ} \mathrm{C}$ under different time intervals.

\subsubsection{Optical simulations}

\subsubsection{Viewing angle and contrast ratio}

Figure 4.13 depicts the optical simulation of the viewing angle characteristics of the fourdomain TN cell, calculated using the extended Jones method. ${ }^{30}$ At $1.25 \mathrm{~V}$ symmetric nature of the isointensity curves were observed, which is typical for multidomain twisted nematic displays. These results are comparable to the experimental results shown in Fig. 4.7. The theoretical calculations and experimental results of the iso-contrast angular dependence (ratio of brightness at $1.25 \mathrm{~V}$ and at $5 \mathrm{~V}$ ) for the four-domain cell are shown in Fig. 4.14. 


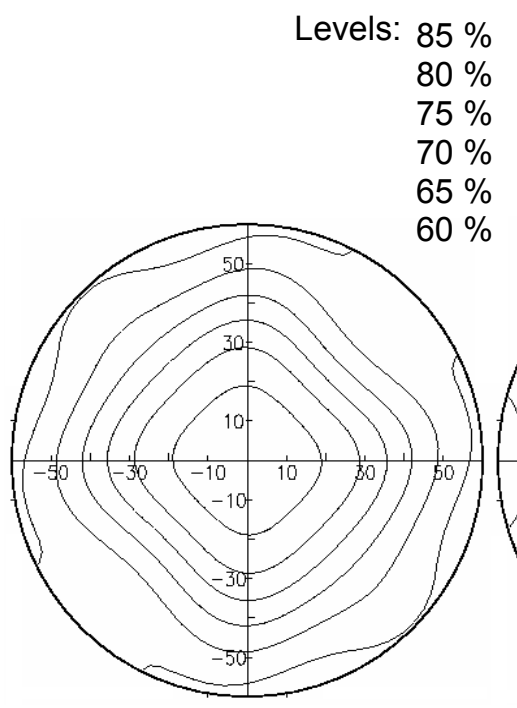

Brightness at $1.25 \mathrm{~V}$

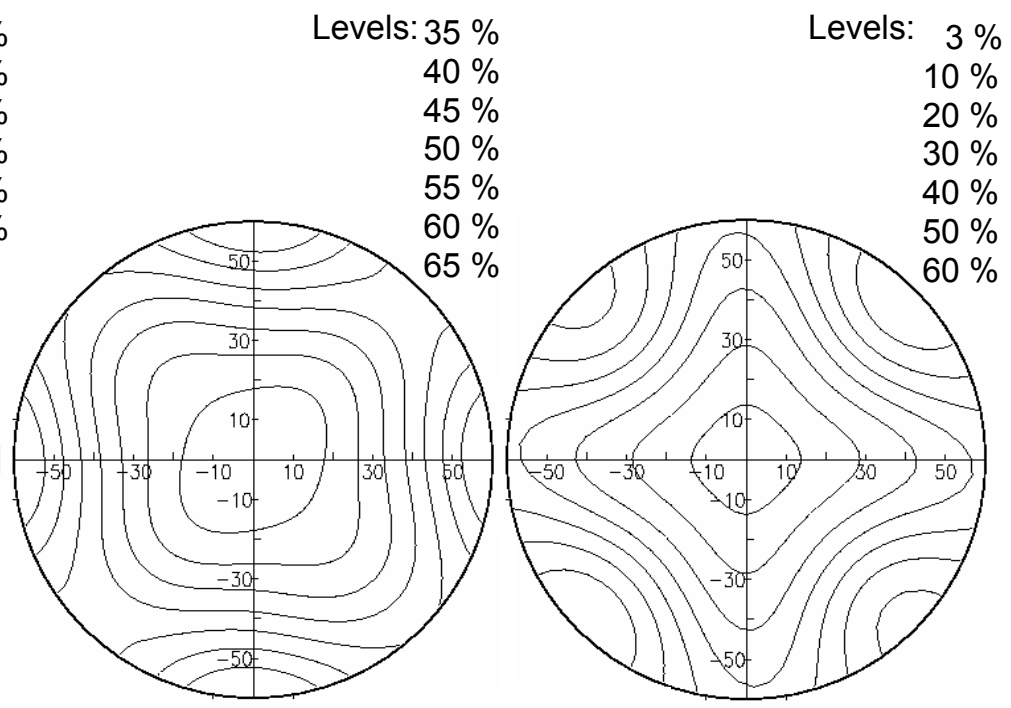

Brightness at $2.0 \mathrm{~V}$

Brightness at $5.0 \mathrm{~V}$

Figure 4.13: The optical simulation results of viewing angle characteristics of the four-domain TN display at $1.25 \mathrm{~V}, 2 \mathrm{~V}$ and $5 \mathrm{~V}$.

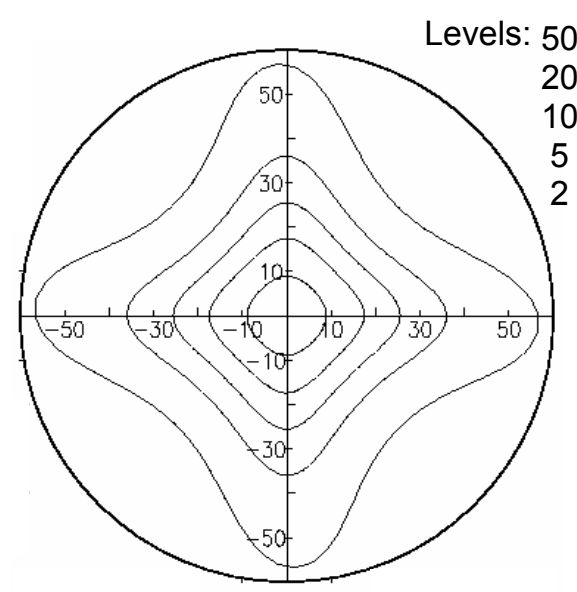

(a)

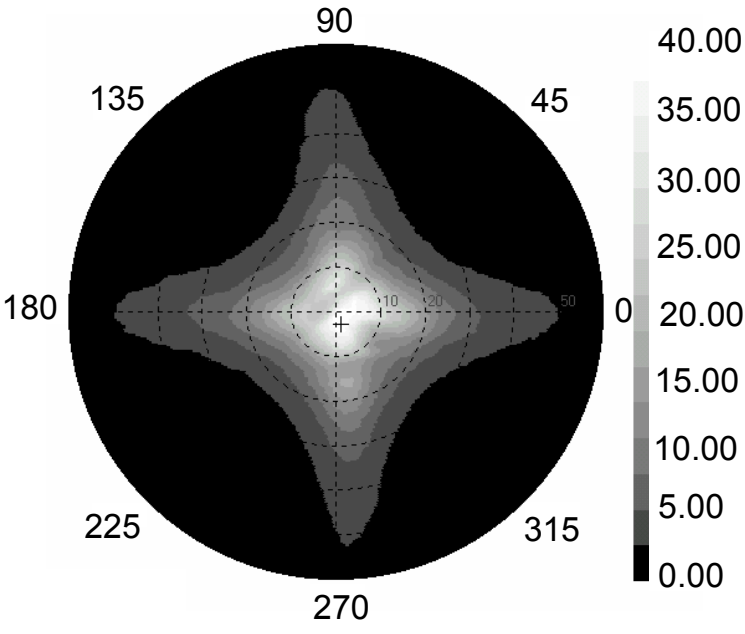

(b)

Figure 4.14: The theoretical calculations (a) and experimental results (b) of the angular dependence of the contrast for the four-domain TN liquid crystal cell. 


\subsubsection{Director orientations}

Figure 4.15 and Fig. 4.16 show the 2D director patterns simulated with the 2dimMOS program. $^{31-33}$ Table 4.1 lists the parameters of the TN cell and the liquid crystal material which was used in the calculations. The figures depict the vertical cross sections of the cell having two sub-pixels. The gray rods represent the directors, where the light end indicates a forward direction and the dark ends indicate a backward direction with respect to the plane of drawing. In this calculation a cross section of a pair of $\mu$-rubbed patterns on the top substrate and a single $\mu$-rubbed pattern on the bottom substrate, which are orthogonal to each other are selected. The left half of the top substrate has a pretilt of $10^{\circ}$ ( $\mu$-rubbed area) with an azimuthal angle of $90^{\circ}$ and the right half has the same pretilt with an azimuthal angle of $270^{\circ}$. The bottom substrate also possesses the same pretilt with an azimuthal angle of $180^{\circ}$.

From the simulations the orientation of directors in an applied field and the formation of disclination lines are observed. In Fig. 4.15 the directors in the twodomains are inclined in opposite directions. At $1.25 \mathrm{~V}$ this results in a disclination line in which the director rotates over $180^{\circ}$, giving rise to dark regions in the transmission as shown in Fig. 4.15 which are comparable with the experimental observations in Fig. 4.6. At higher voltage the disclination lines disappear, to give a perfectly dark state (homeotropic state) as shown in Fig. 4.16. 

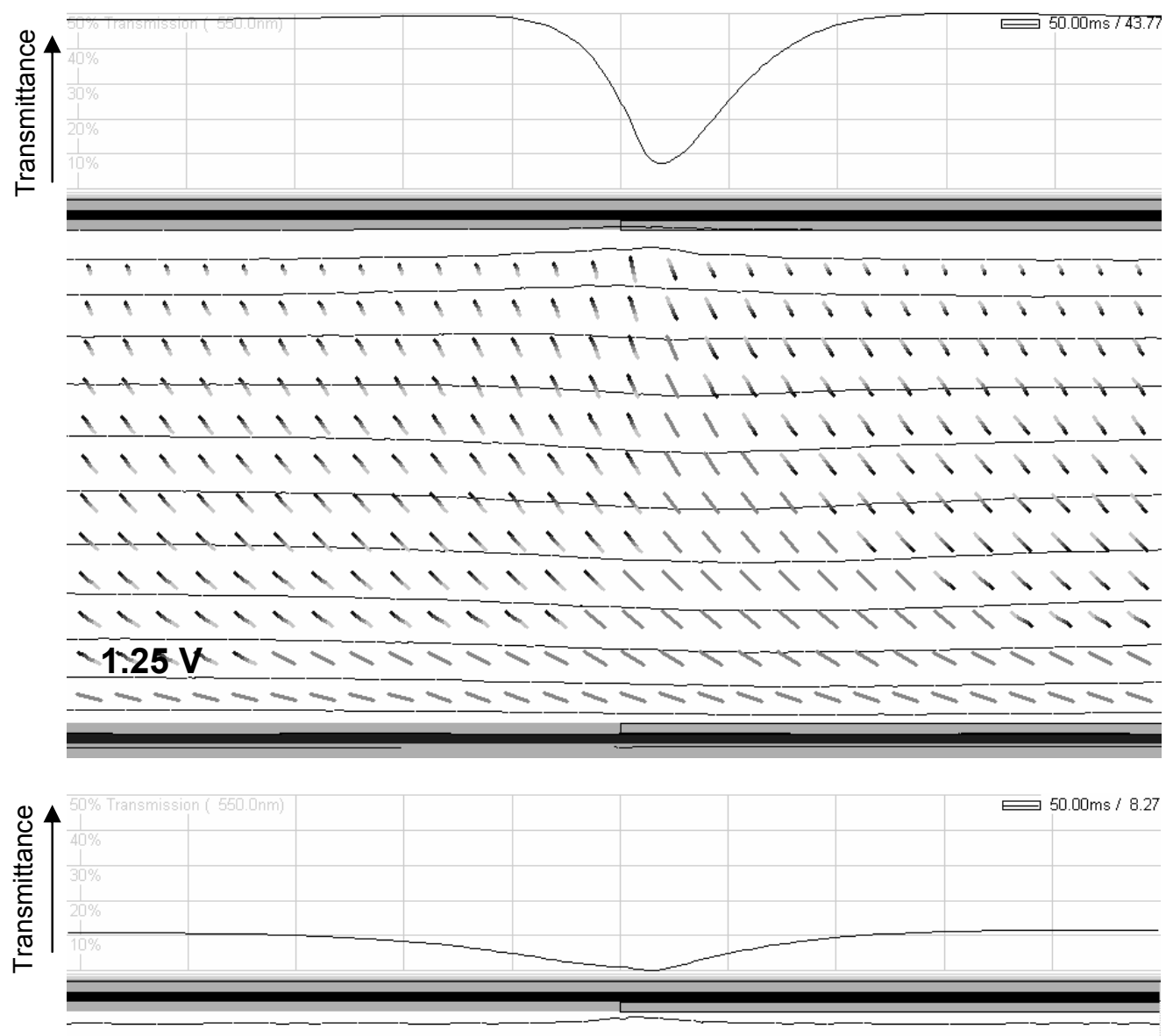

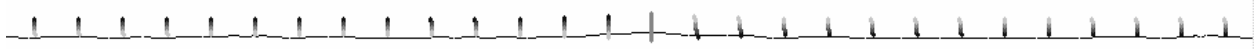

$\begin{array}{lllllllllllllllllllllllllll}1 & 1 & 1 & 1 & 1 & 1 & 1 & 1 & 1 & 1 & 1 & 1 & 1 & 1 & 1 & 1 & 1 & 1 & 1 & 1 & 1\end{array}$

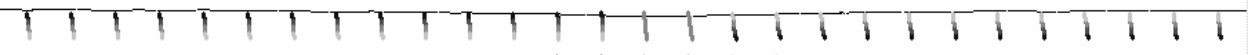
$-11111 \frac{1}{1} 1 \frac{1}{1} 1 \frac{1}{1} 1 \frac{1}{1} 1111111111111111$ 1111111111111111111111111111 1111111111111111111111111111 1111111111111111111111111111 111111111111111111111111111111

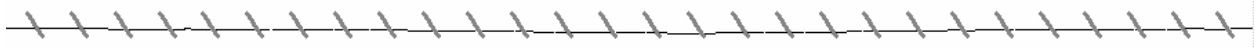

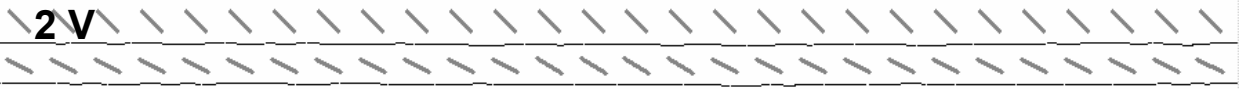

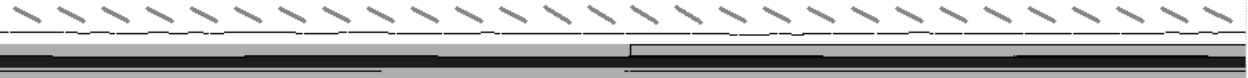

Figure 4.15: Results of 2dimMOS calculation for the director orientation of four-domain cell at 1.25 and $2 \mathrm{~V}$. Top left pretilt $=10^{\circ}$ azimuth $=90^{\circ}$, top right pretilt $=10^{\circ}$ azimuth $=270^{\circ}$, bottom pretilt $=10^{\circ}$ azimuth $=180^{\circ}$. Calculated transmittance over the same section is plotted at the top. 


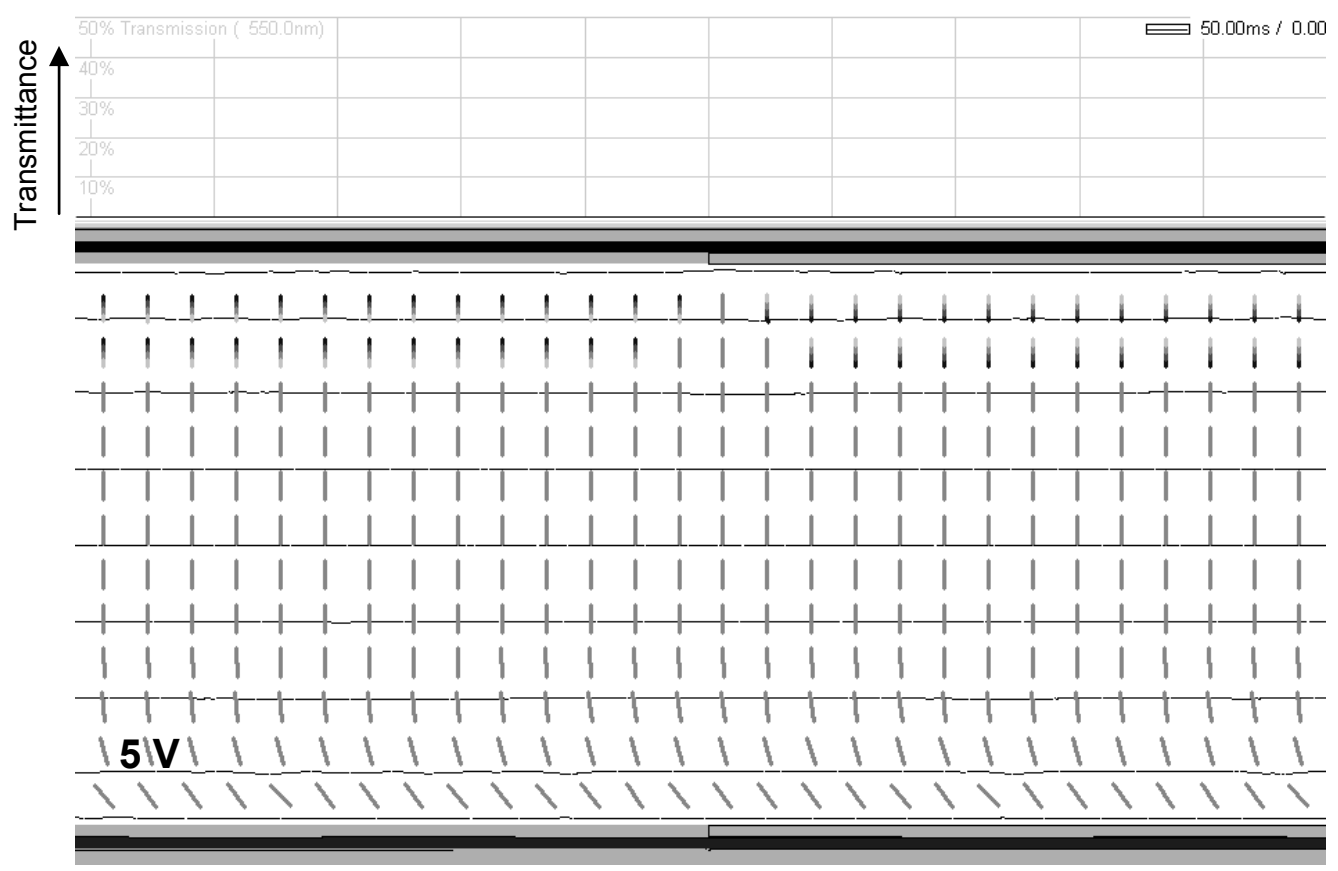

Figure 4.16: Results of 2dimMOS calculation for the director orientation of four-domain cell at $5 \mathrm{~V}$. Top left pretilt $=10^{\circ}$ azimuth $=90^{\circ}$, top right pretilt $=10^{\circ}$ azimuth $=270^{\circ}$, bottom pretilt $=$ $10^{\circ}$ azimuth $=180^{\circ}$. Calculated transmittance over the same section is plotted at the top.

Table 4.1: The parameters of TN cell used in the optical simulations. (The material parameters of LC E7 are used.)

\begin{tabular}{cll}
\hline \hline & & \\
Dielectric anisotropy & $\varepsilon_{/ /}$ & 19.0 \\
$\left(20{ }^{\circ} \mathrm{C}, 1 \mathrm{kHz}\right)$ & $\varepsilon_{\perp}$ & 5.2 \\
& $\Delta \varepsilon$ & +13.8 \\
Elastic constants $\left(20^{\circ} \mathrm{C}\right)$ & $k_{11}$ & $11.1 \mathrm{pN}$ \\
& $k_{33}$ & $17.1 \mathrm{pN}$ \\
& $k_{33} / k_{11}$ & 1.54 \\
Optical anisotropy & $\Delta n$ & 0.2253 \\
$\left(20^{\circ} \mathrm{C}, 589 \mathrm{~nm}\right)$ & $n_{e}$ & 1.7464 \\
& $n_{o}$ & 1.5211 \\
Cell gap $(\mu \mathrm{m})$ & $d$ & 5.0 \\
Pretilt angle & $\theta_{p}$ & $10^{\circ}$ \\
\hline
\end{tabular}



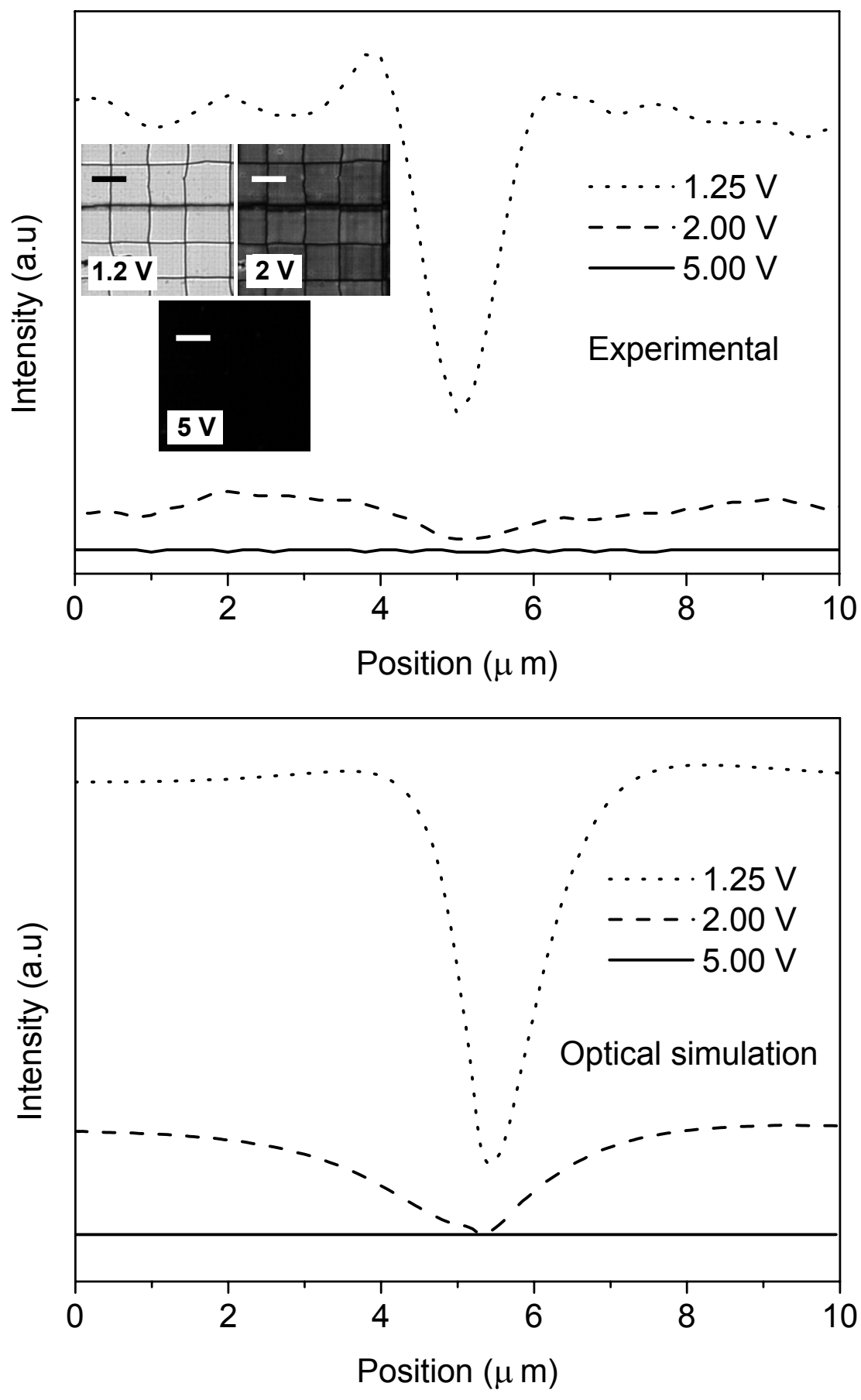

Figure 4.17: Comparison of the experimental and calculated intensity profile at different voltages. Optical microscopy picture of the disclination lines at the same voltages are also shown, the dark line (top left) shows the scanned area. 
Figure 4.17 shows the line scans of the optical microscopy image at $1.25 \mathrm{~V}, 2 \mathrm{~V}$ and $5 \mathrm{~V}$. For the line scans a straight line was drawn over the image across the disclination lines and the intensity vs. position was plotted. The experimental and optical simulation results are in good agreement. At $1.25 \mathrm{~V}$ the disclination lines appear due to the difference in the orientation of the directors in each sub-pixel. A slight asymmetry is seen in the curve. The intensity vs. position changes more steeply on the left side of the disclination since the twist helps to align the directors in the plane of drawing, parallel to one of the polarizer directions (see Fig. 4.15).

\subsubsection{Electro-optical characteristics}

Figure 4.18 shows a comparison of transmission-voltage characteristics of the fourdomain sample (pretilt, $9.8^{\circ} \pm 0.5$ ) to a conventional $\mathrm{TN}$ cell (with $2.65^{\circ} \pm 0.5$ pretilt angle).

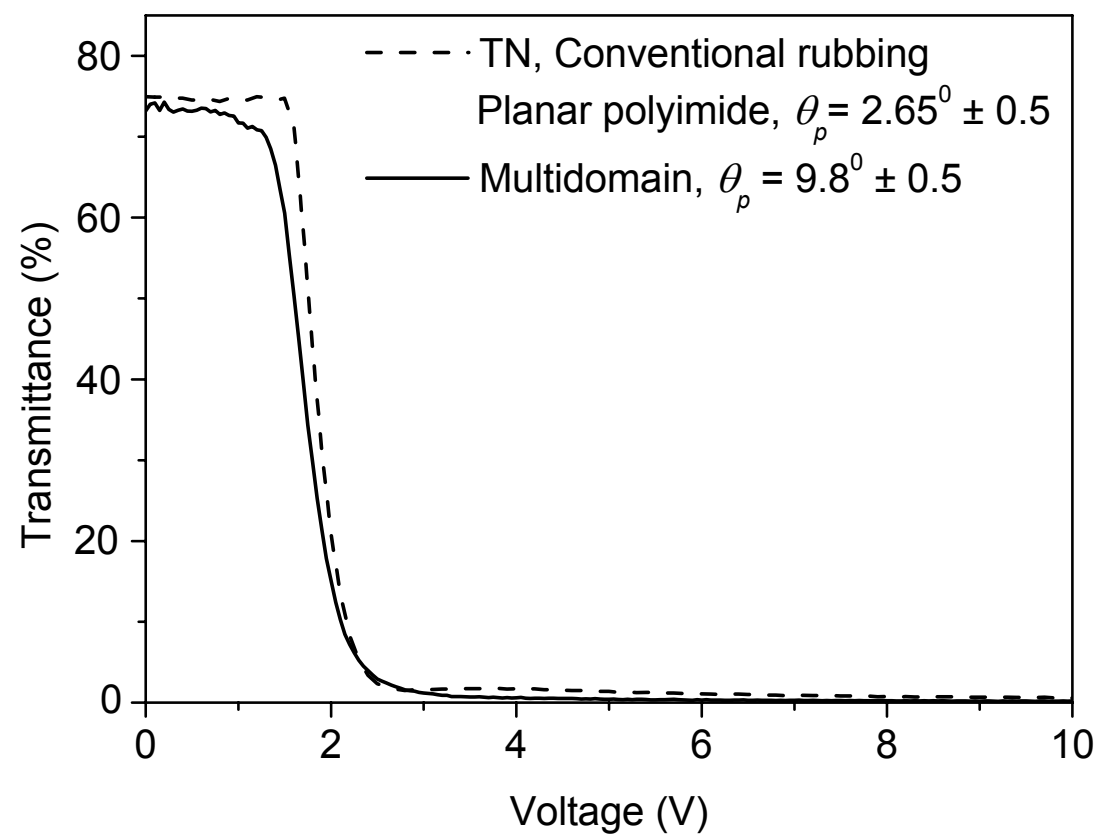

Figure 4.18: Transmission-voltage curves of a conventional TN cell compared to a four-domain TN display. 
There are two notable features that standout in Fig. 4.18. First the steepness of the transmission voltage curve of the four-domain is "softened" due to the large pretilt. Second, the brightness is lower in the four-domain sample due to the disclination lines. Both of these effects are expected as a consequence of four-domain alignment.

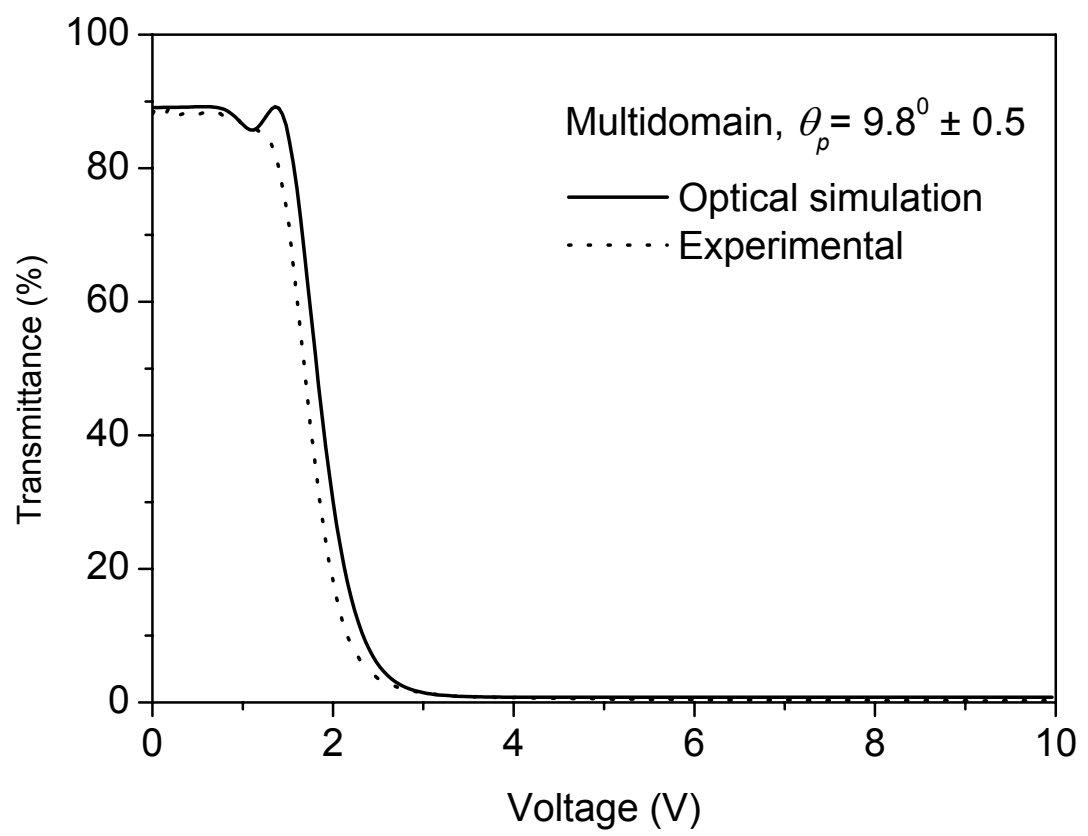

Figure 4.19: Experimental and simulated transmission-voltage curves at normal incidence of a four-domain twisted nematic cell.

The transmission-voltage curve was further calculated using the extended Jones matrix method for the large pretilt sample in Fig. 4.19. The experimental and optical simulation results are in good agreement.

The stability of the four-domain purely depends on its pretilt angle. In this fourdomain structure a voltage initialization process is required. The observation of the symmetric nature of the iso-intensity curves for the multidomain structure is promising for the fabrication of real display modules. In this study the symmetric nature of the viewing angle is restricted to $60^{\circ}$, but it can be further widened using suitable optical compensation film. The observed low color dispersion for this multidomain structure also opens the possibility for full color display panels. 


\subsection{Conclusions}

In this chapter a multidomain twisted nematic structure was fabricated using a reverse $\mu$ rubbing process. The $\mu$-rubbing process can be used to generate a multidomain configuration with a high surface pretilt angle, in this case $\sim 10^{\circ}$, using a $150 \mathrm{~g}$ load with a $1 \mathrm{~mm}$ metallic sphere, with the possibility of higher pretilt angles for lower loads. The four-domain structure is not stable in the zero voltage state since the surface pretilt angle is below that needed for a stable structure for given pixel dimensions and cell gap, it only becomes stable at high voltages. The optical simulation results are in accordance with the experimental observations. The $2 \mathrm{D}$ modeling of the director pattern and the viewing angle measurements are useful for a better understanding of their electro-optical properties. This $\mu$-rubbing technique is straightforward, flexible, economical and simple. This technique can be scaled up and used in the production of large area liquid crystal displays.

\subsection{References}

[1] S. W. Depp and W. E. Howard, Sci. Am. 266, 40 (1993).

[2] S. Musa, Sci. Am. 277, 87 (1997).

[3] N. Yamagishi, H. Watanabe, K. Yokoyama, SID89 Digt. 316 (1989).

[4] Y. Yamagnchi, T. Miyashita, T. Uchida, SID93 Digt. 277 (1993).

[5] H. Mori, Jpn. J. Appl. Phys. 36, 143 (1997).

[6] H. Mori, Y. Itoh, T. Nishiura, T. Nakamura, Y. Shinagawa, Jpn. J. Appl. Phys. 36, 143 (1997).

[7] P. van de Witte, S. Stallinga, J. A. M. M van Haaren, Jpn. J. Appl. Phys., Part 1, 39, $101(2000)$.

[8] C. Joubert, J. C. Lehureau, L. F. D. Lee, B. Morbieu, SPIE proceeding, San Jose 3635, 137 (1999).

[9] J. Li, E. S. Lee, H. Vithana, P. J. Bos, Jpn. J. Appl. Phys. 35, L1446 (1996).

[10] H. Yoshida, T. Seino, Y. Koike, Jpn. J. Appl. Phys. 36, L1449 (1997).

[11] K. H. Yang, Jpn. J. Appl. Phys. 31, L1603 (1992). 
[12] H. Vithana, D. Johnson, P. Bos, R. Herke, Y. K. Fung, S. Jamal, Jpn. J. Appl. Phys. 35, 2222 (1996).

[13] M. Schadt, K. Schmitt, V. Kozinkhov, V. Chigrinov, Jpn.J.Appl.Phys. 31, 2155 (1992).

[14] M. Schadt, H. Seiberle, A. Schuster, Nature 381, 212 (1996).

[15] P. J. Shannon, W. M. Gibbons, S. T. Sun, Nature 368, 532 (1994).

[16] K. H. Yang, Jpn. J. Appl. Phys. 31, 1603 (1992).

[17] C. Nieuwkerk et al, SID2000 Digt. XXXI, 842 (2000).

[18] D. S. Seo, J. Y. Hwang, Jpn. J. Appl. Phys. 39, L914 (2000).

[19] K. Ohmuro, S. Kataoka, T. Saski, Y. Koike, SID97 Digt. 845, (1997).

[20] D. S. Seo, J. Y. Hwang, Jpn. J. Appl. Phys. 38, L1432 (1999).

[21] R. A. Soref, J. Appl. Phys. 45, 5466, (1974).

[22] M. Oh-e, K. Kondo, Appl. Phys. Lett. 67, 3895 (1995).

[23] M. Oh-e, K. Kondo, Appl. Phys. Lett. 69, 623 (1996).

[24] M. Schadt, H. Seiberle, A. Schuster, Nature 381, 212 (1996).

[25] J. Chen, P. J. Bos, D. L. Johnson, D. R. Bryant, J. Li, S. H. Jamal, J. R. Kelly, J. Appl. Phys. 80, 1985 (1996).

[26] J. Chen, P. J. Bos, D. R. Bryant, D. L. Johnson, S. H. Jamal, J. R. Kelly, Appl. Phys. Lett. 67, 1990 (1995).

[27] G. P. Sinha, C. Rosenblatt, L. V. Mirantsev, Phys. Rev. E 65, 041718 (2002).

[28] B. Wen, M. P. Mahajan, C. Rosenblatt, Appl. Phys. Lett. 76, 1240 (2000).

[29] B. L. V. Horn, H. H.Winter, Appli. Opti. 40, 2089 (2001).

[30] P. Yeh, L. Gu, Optics of liquid crystal displays, John Wiley \& Sons, New York, Chapter 8 (1999).

[31] P. G. de Gennes, J. Prost, The physics of Liquid Crystals, Oxford University Press (1994).

[32] M. E. Becker, H. Wohler, M. Kamm, J. Kreis, Proceedings of SID 96, 596 (1996).

[33] 2dimMOS, www.autronic-melchers.com. 


\section{Chapter 5}

\section{Four-Domain Twisted Vertically Aligned Liquid Crystal Display Using $\mu-R u b b i n g *$}

\subsection{Introduction}

As discussed in Chapter 1, the vertically aligned display mode is used in current flat panel displays like television (LCD-TVs) and computer monitors. With the commercial arrival of LCD-TVs, there is a compelling need for sub-pixel patterning techniques that can be performed on large area displays in a simple and inexpensive way to improve the viewing angle and gray-scale inversion. Current techniques used to fabricate vertical alignment (VA) and multidomain VA displays ${ }^{1}$ for wide viewing angle include: fourdomain alignment based on ridges applied underneath the alignment layer, ${ }^{2}$ fringe field structures, ${ }^{3}$ vacuum deposition techniques, ${ }^{4}$ photolithographic techniques, ${ }^{5}$ etc. These techniques are process intensive and are therefore rather expensive to implement on large area displays.

In Chapter 3 and 4, it was shown that the microrubbing of homeotropic alignment layers results in pretilt between $9^{\circ}$ and $16^{\circ}$. Moreover, it was also shown that these moderately high pretilt can be used to create a multidomain TN-LCD with a stable, multidomain sub-pixel structure at voltages above $1.25 \mathrm{~V}$. In this chapter, the $\mu$-rubbing rubbing of homeotropic alignments layers is re-addressed. More specifically, it is attempted to further increase the pretilt angle to obtain stable multidomain twisted vertically aligned display with a stable multidomain structure at all voltages. Therefore a different homeotropic polyimide was selected than was used in Chapter 4.

*This chapter is partly reproduced from: S. Varghese, G. P. Crawford, C. W. M. Bastiaansen, D. K. G. de Boer, D. J. Broer, Appl. Phys. Lett. (in press), (2005). 


\subsection{Experimental}

\subsubsection{Materials}

The ITO coated glass substrate was spin coated $(5 \mathrm{~s}$ at $1000 \mathrm{rpm}$ followed by $40 \mathrm{~s}$ at 5000 rpm) with a homeotropic polyimide precursor, Sunever SE-1211, Nissan Chemical Industries, Ltd. This polyimide is known to support homeotropic boundary conditions. It was then preheated to $100{ }^{\circ} \mathrm{C}$ for 10 minutes and imidized at $180{ }^{\circ} \mathrm{C}$ under vacuum for 90 minutes to obtain a transparent film with a thickness of $\sim 150 \mathrm{~nm}$. The liquid crystals MLC-6610, MLC-7026, both exhibiting a negative dielectric anisotropy needed to switch vertically aligned liquid crystals, and chiral dopant ZLI-811 (Merck Ltd., Darmstadt, Germany) was used throughout the experiments.

\subsection{2 $\mu$-Rubbing setup}

This polyimide film was $\mu$-rubbed using a metallic sphere $(1 \mathrm{~mm}$ diameter) (see Chapter 4 , experimental section). The metallic sphere was in direct contact with the alignment layer as it traversed across the alignment layer (substrate) creating micrometer sized rubbed lines. The velocity of the metallic sphere was $10 \mathrm{~mm} /$ minute at a pre-specified load of $150 \mathrm{~g}$ at room temperature. The $\mu$-rubbing technique was used to create multidomain pixels by rubbing the surface in such a way that neighboring alignment regions were rubbed in opposite directions.

\subsubsection{Cell Construction}

Liquid crystal cells were constructed by orienting two $\mu$-rubbed substrates orthogonal to each other, separated by $5 \mu \mathrm{m}$ spacers and securing them with UV curable glue with the edges sealed along the sample periphery. For the twisted vertically aligned (TVA) mode liquid crystal MLC-6610 was used as it is, and for the frustrated twisted vertically aligned (FTVA) mode MLC-6610 along with a calculated amount (0.5\%) of chiral dopant ZLI811 was employed. The $\mathrm{LC}$ cells were filled by capillary action at $100^{\circ} \mathrm{C}\left(\sim 20^{\circ} \mathrm{C}\right.$ above the nematic-isotropic transition temperature). 


\subsubsection{Characterization}

The alignment of liquid crystals was studied using polarized light microscopy (Zeiss LM Axioplan). The pretilt angle was measured directly using the crystal rotation method (Autronic, TBA 107). The crystal rotation method ${ }^{6}$ was used to measure the surface pretilt angle on a sample having an $18 \mu \mathrm{m}$ cell gap with antiparallel rubbing directions. For this measurement antiparallel cell with $18 \mu \mathrm{m}$ cell gap was constructed by recording parallel with same rubbing directions touching each other. Electro-optical measurements were performed on a DMS 703 display measuring system (Autronic-Melchers Gmbh). Iso-contrast and chromaticity measurements were performed using Eldim conoscope. The $\mu$-rubbed patterns were investigated by atomic force microscopy (AFM) (Nanoscope IIIa, Digital Instruments, Santa Barbara, California) equipped with conventional $\mathrm{Si}_{3} \mathrm{~N}_{4}$ cantilevers and tips in tapping mode. The cell thickness was determined by UV-visible spectroscopy (Shimadzu UV-3102 PC).

\subsection{Results and discussion}

\subsubsection{Surface characterization}

Fabrication and characterization of the four-domain twisted vertically aligned cell used the same experimental conditions and setup as the $\mu$-rubbed alignment layers described in Chapter 4. The $\mu$-rubbed surface was characterized by AFM, Fig. 5.1 shows the AFM image of the surface and its height profile (top). During the $\mu$-rubbing process micro grooves were formed with a depth in the range of 5-15 $\mathrm{nm}$ and with a line width of 45 $\mu \mathrm{m}$. The root mean square surface roughness of the $\mu$-rubbed lines was found to be $8 \pm 2$ $\mathrm{nm}$. A small amount of debris was formed during the $\mu$-rubbing process as can be seen in the AFM image. 


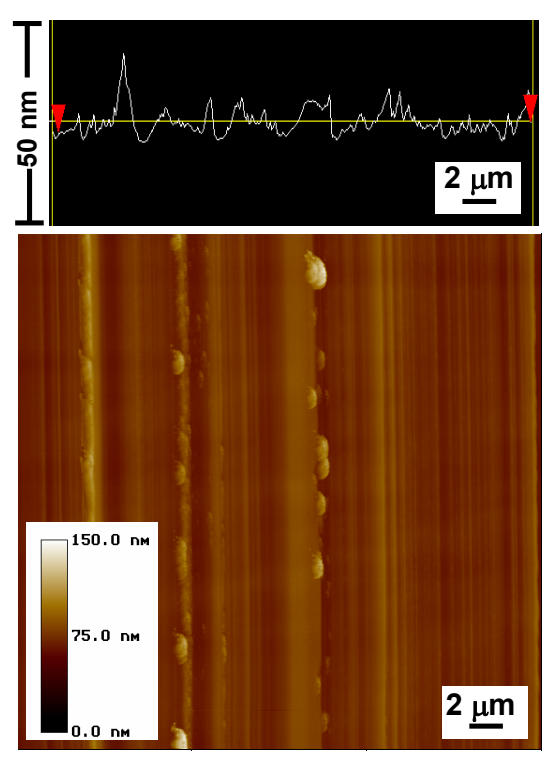

Figure 5.1: Atomic force microscopy image of the $\mu$-rubbed polyimide surface and its height profile (top).

The pretilt angle of the $\mu$-rubbed alignment layers was measured at $89^{\circ}$ using the crystal rotation method (see Chapter 2). The pretilt of the specific homeotropic alignment layer used here (Sunever SE-1211) was very high in comparison to the homeotropic polyimide used in Chapter 4 (AL-75114) where the pretilt was measured between $7^{\circ}$ and $16^{\circ}$. The same experimental conditions were used for the production of the $\mu$-rubbed regions and consequently the large differences in pretilt probably originate from differences in the chemical and/or physical properties of the polyimide alignment layers. Unfortunately, the chemical structures of the polyimide alignment layers cannot be obtained from the manufacturer therefore, the exact origin of the large pretilt variation is hard to pinpoint. However, it is expected that the large pretilt differences originate from differences in the crosslink density of the polyimide alignment layers or in differences in the length and/or chemical nature of the aliphatic side chains at the outer surface of the alignment layer. In both cases, a difference is expected in the plastic deformation of the surface of the polyimide alignment layers during $\mu$-rubbing which influences the pretilt angle of LCs. 


\subsubsection{Director orientations}

A schematic drawing is shown of the nematic director $\bar{N}$ in electro-optical cell with a pretilt angle of $\theta_{p}$ from the normal in Fig. 5.2. In the cell, the $\mu$-rubbing directions of the top and bottom substrates are orthogonal to each other. In an electric field, the small deviation in pretilt from the normal $\left(1^{\circ}\right)$ switches the nematic directors to the respective direction in an applied electric field giving rise to the formation of a four-domain structure.

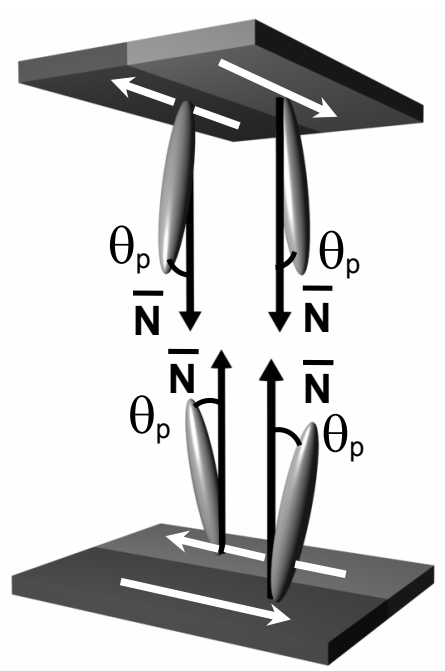

Figure 5.2: The rub directions of the top and bottom substrates in the liquid crystal cell and the orientation of nematic directors.

The four-domain twisted vertically aligned liquid crystal display (TVA-LCD) in the on-state and in the off-state is shown in Fig. 5.3. At zero voltage a homeotropic state is obtained with a small but well defined unidirectional pretilt angle. When a voltage is applied the molecules align perpendicular to the electric field direction since the material possesses a negative dielectric anisotropy $(-\Delta \varepsilon)$. Since the pretilt angle of the four regions of a pixel is uniquely defined by the $\mu$-rubbing process, the four midplane tilt angles are pointing in different directions. In the off-state the sample is expected to be perfectly black since there are no domain boundaries in this state. 

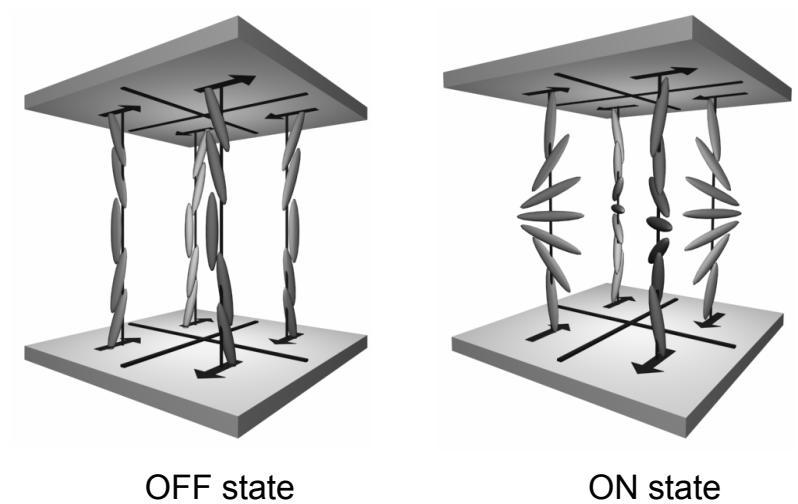

ON state
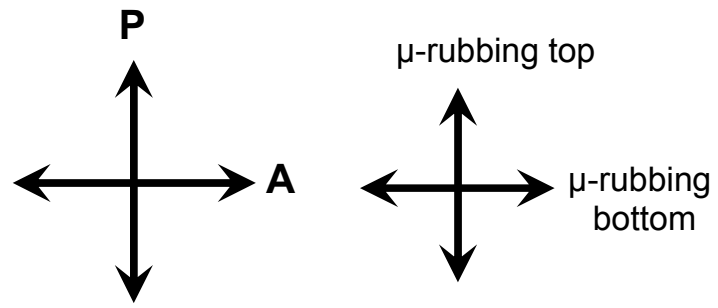

Figure 5.3: The twisted vertically aligned (TVA) mode is schematically illustrated in the OFF and $\mathrm{ON}$ state.

To study the influence of a chiral dopant on the twist sense of the TVA mode, a cell was filled with $-\Delta \varepsilon$ liquid crystal modified with a calculated amount of chiral dopant for $90^{\circ}$ twist. The addition of a chiral dopant with negative twisting power in the LC directs the molecules to a left handed twist. The addition of chiral dopant in this twisted vertically aligned system creates a frustration in two diagonal pixels due to their opposite twist. The material is doped with a chiral dopant as disclosed earlier by Crandall and coworkers. $^{7}$ In this case $0.5 \%$ of the chiral dopant ZLI-811 was used to create a $\sim \frac{\pi}{4}$ twist upon application of an electric field. Figure 5.4 illustrates the four-domain frustrated twisted vertically aligned LCD (FTVA-LCD) state in the off-state (zero voltage) and on-state (high voltage). 


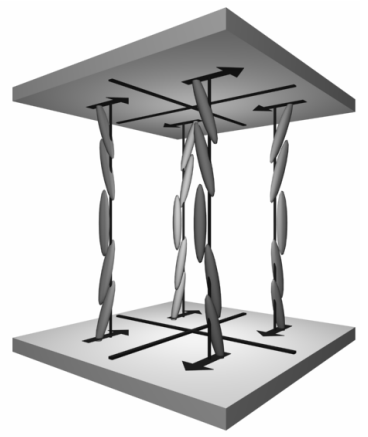

OFF state

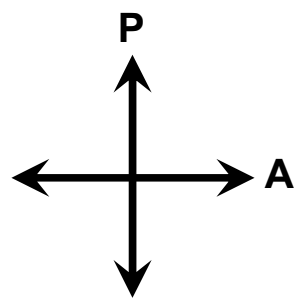

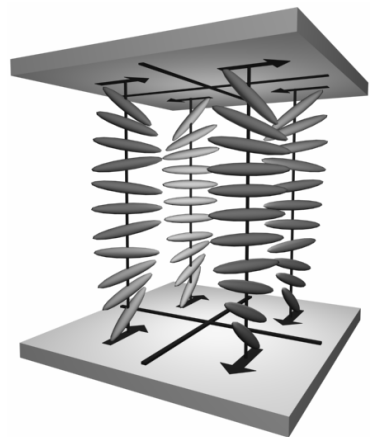

ON state

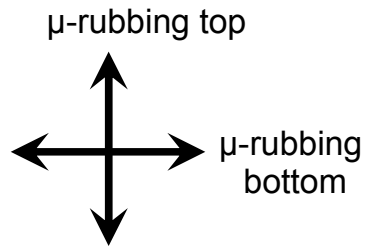

Figure 5.4: Schematic representation of the frustrated twisted vertically aligned (FTVA) mode in the $O F F$ and $O N$ state.

In contrast to the conventional four-domain structures using planar alignment with two right handed and two left handed sub-pixels, the mode presented here has all domains of the same handedness due to the addition of chiral dopant.

\subsubsection{Viewing angle and contrast ratio}

Optical microscopy pictures and the corresponding isointensity curves of the TVA and FTVA mode are shown in Fig. 5.5. The isointensity curves were measured at different voltages and the specific polarization and rubbing directions are depicted in Fig. 5.3 and Fig. 5.4. The symmetric nature of the isointensity curve is visible at higher voltages, which clearly show the attractiveness of this TVA configuration for wide viewing angle applications. The sub-pixel size is approximately $45 \times 45 \mu \mathrm{m}^{2}$ and the domain boundaries are black. 


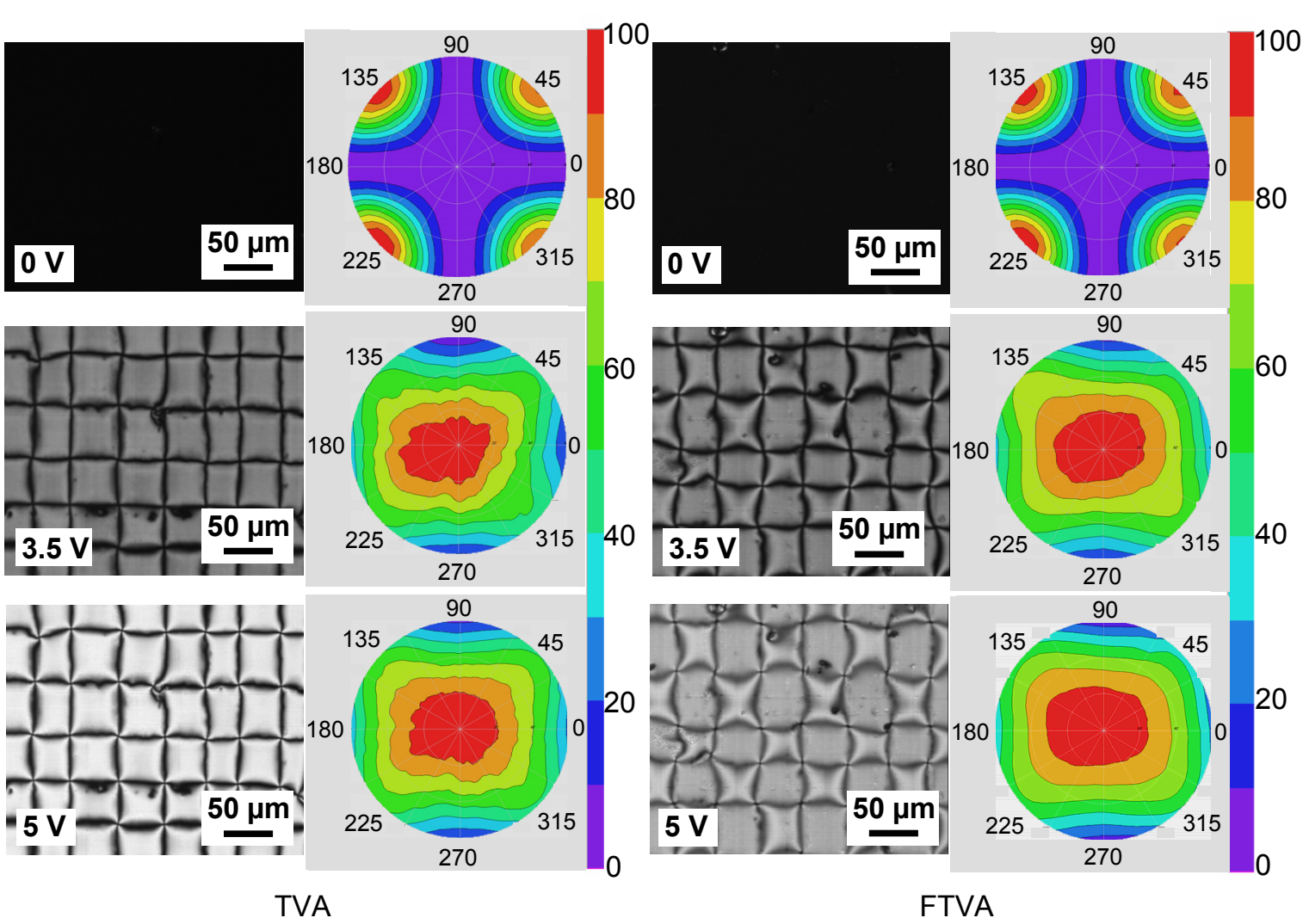

Figure 5.5: Polarizing microscope images and the corresponding iso-intensity curves of the TVA (left) and FTVA mode (right) under different voltages created by $\mu$-rubbing process.

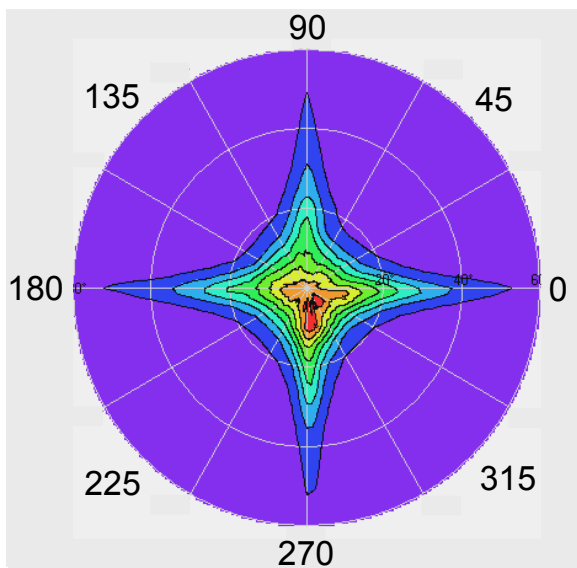

Contrast ratio - TVA

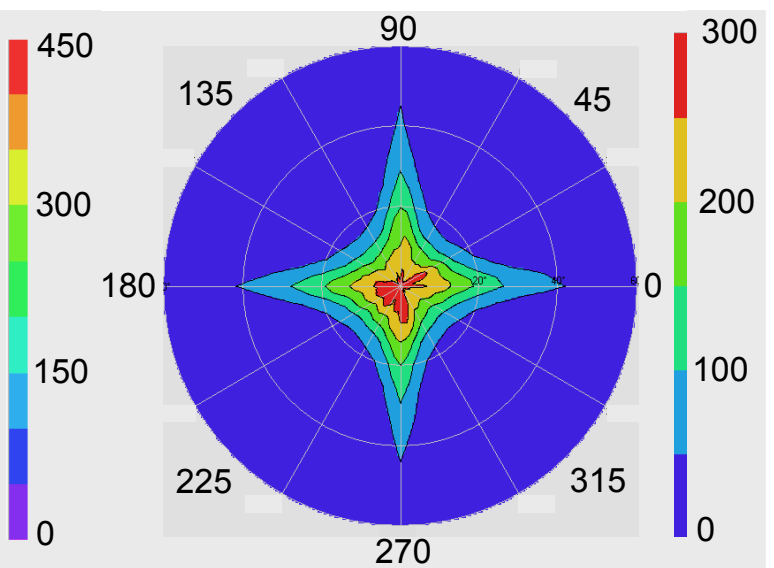

Contrast ratio - FTVA

Figure 5.6: The iso-contrast measurement of the TVA and FTVA cell. 
In conventional pixels, two right handed and two left handed sub-pixels exist. The addition of chiral dopant with negative twisting power in FTVA creates two subpixels along a diagonal to twist easily, the other two sub-pixels become frustrated due to the innate opposite twist sense. The frustration may lead to a different electrical response of the liquid crystal director upon application of an electrical field. This explains the slight difference in optical polarizing microscope observation (Fig. 5.5, right part) where the two frustrated sub-pixels can be distinguished from the non-frustrated ones, where as the non-frustrated sub-pixels compare to the pixels in the TVA mode (Fig. 5.5, left part).

Both configurations (TVA and FTVA) exhibit an excellent and highly symmetrical viewing angle. The viewing angle characteristics of the multidomain cell with the chiral dopant are excellent in comparison to a classical twisted nematic cell. The excellent viewing angle of both kinds of cells in the on-state originates from the averaging effect of the liquid crystal directors in the sub-pixels in the four-domain.

A common problem of all vertically aligned displays, is the significant amount of light leakage in the zero voltage state due to the optical retardation at oblique angles. ${ }^{8}$ The multidomain TVA or the FTVA displays also exhibit this light leakage at large angles (see figure 5.5 top image). However, because of its symmetry, a simple negative c-plate compensation, providing an optical anisotropy complementary to that of the vertically aligned liquid crystals (which behaves as a positive $c$-plate) can be used to minimize the light leakage and improve the viewing angle volume in the zero voltage state. ${ }^{9}$ Polymer films that are biaxially stretched or are based on discotic materials provide this function.

The angular dependence of the contrast ratios of the four-domain TVA mode and FTVA mode cells are shown in Fig. 5.6. A symmetric iso-contrast plot is observed in both cases. The FTVA mode is less black compared to the TVA mode due to the presence of frustrations in domains with right hand twist, which results in a lower contrast ratio. 
Figure 5.7 depicts the intensity profile of the four-domain pixel, as obtained from optical microscopy, for the TVA and FTVA mode. The intensity profile of the TVA mode is slightly higher than that of the FTVA mode. This can be explained by the presence of frustrated domains due to the presence of chiral dopant with negative twisting power as mentioned earlier.

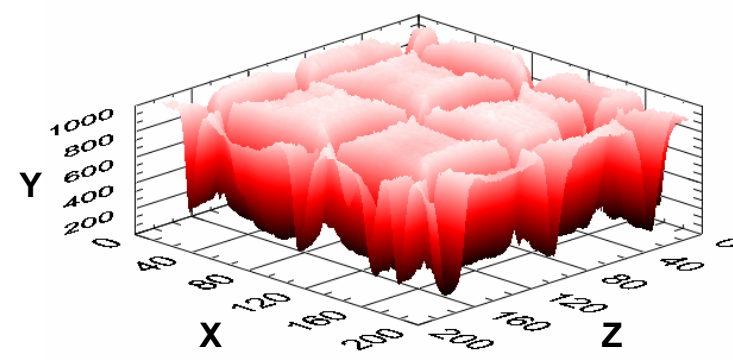

TVA mode

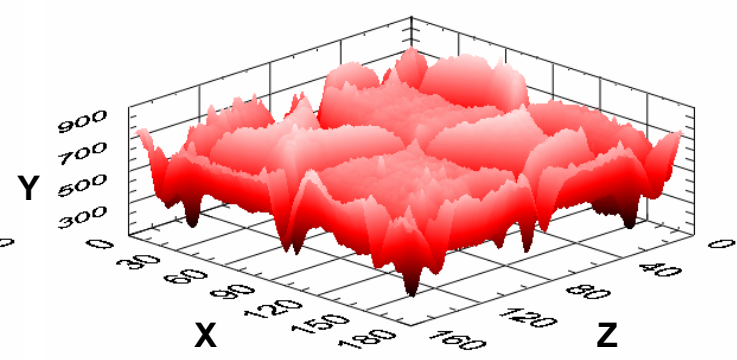

FTVA mode

Figure 5.7: Intensity profile of the four-domain structure in the TVA mode (left) and the FTVA mode (right). $X, Y, Z$ coordinates are in pixel units.

\subsubsection{Electro-optical characterization}

Figure 5.8 shows the transmission-voltage $(\mathrm{T}-\mathrm{V})$ curve of vertically aligned four-domain pixel with and without chiral dopant. A traditional T-V curve is obtained with no on-axis light leakage in the off-state, therefore resulting in an extremely high on/off contrast ratio of 580:1 and 540:1 for normal viewing TVA and FTVA mode respectively. The inset in Fig. 5.8 shows the dynamic response times (measured between $10 \%$ and $90 \%$ transmission) for the TVA mode and FTVA mode. As expected, in both the TVA mode and FTVA mode, the off-time (relaxation) is essentially independent of voltage. For the TVA mode, the on-time scales roughly with the inverse of voltage as expected from the theory. ${ }^{10}$ 


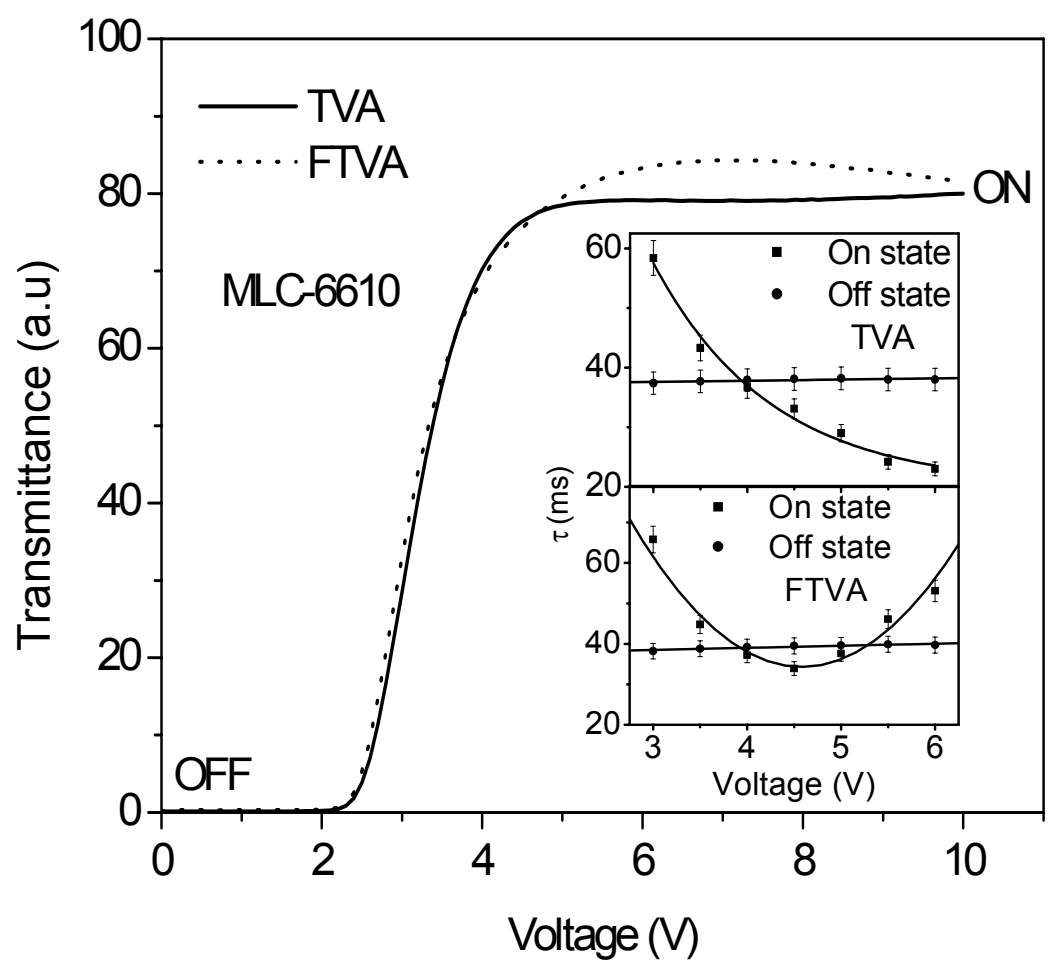

Figure 5.8: Transmission-voltage curve for the bend and twist mode vertically aligned fourdomain cell and their corresponding response times in the inset.

However, in the FTVA mode there is a reproducible increase in the on-time above $4.5 \mathrm{~V}$. Experiments suggest that such a phenomenon may be attributed to twisting beyond the desired $\frac{\pi}{4}$ twist angle (too much chiral dopant). This is analogous to the situation in super twisted nematic display, where with increasing twist (more chiral dopant), response times also increase. ${ }^{11}$ Alternatively, it is possible that the formation of frustrated domains cost more time than that of non-frustrated ones.

In Fig. 5.9 the TV-curves are shown of the TVA mode for the three primary colors red $(650 \mathrm{~nm})$, green $(550 \mathrm{~nm})$ and blue $(450 \mathrm{~nm})$ at normal incidence. The optical characteristics are dependent on the wavelength and this is the consequence of the wavelength dependence of the phase retardation, $\delta=2 \pi d \Delta n / \lambda$. So for vertically aligned LCDs different voltages have to be used for the different colors. 


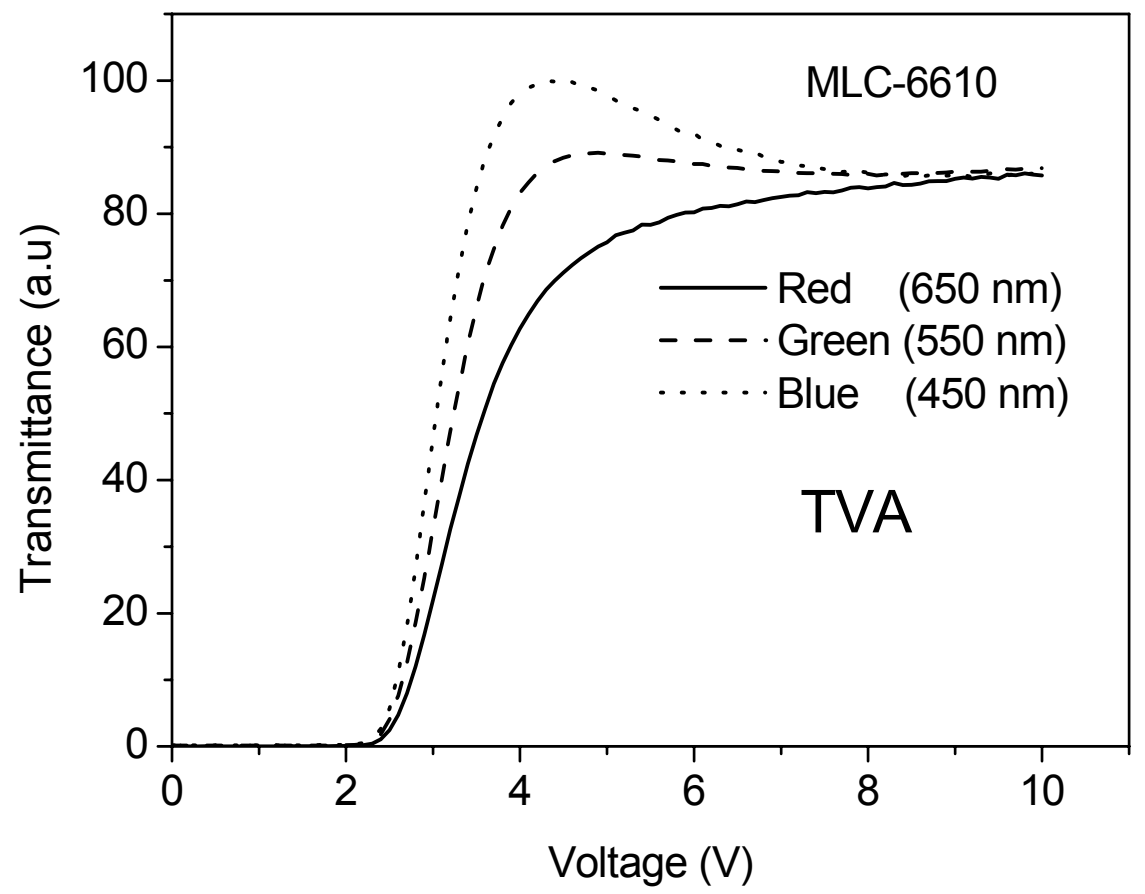

Figure 5.9: Transmission-voltage curve at normal incidence of a vertically aligned four-domain liquid crystal display (TVA) at different wavelength.

Figure 5.10 shows the comparison of transmission-voltage characteristics of two different liquid crystals, MLC-6610 and MLC-7026 with different dielectric anisotropy $(-\Delta \varepsilon)$, and both switched in the TVA mode. Rather fast switching kinetics is observed in the case of MLC-7026 (if compared with MLC-6610). The liquid crystal mixture MLC7026 possesses a low rotational viscosity and low dielectric constant compared to MLC6610. This kind of liquid crystals is useful in LCD-TV applications that require fast switching kinetics and high resolution for fast moving images.

Inset in Fig. 5.10 shows the comparison of dynamic response time (measured between 10\% and 90\% transmission) for MLC-6610 and MLC-7026. There is only a slight difference in the off-state and is independent of change in voltage and the on-time scales roughly with the inverse of voltage as expected. 


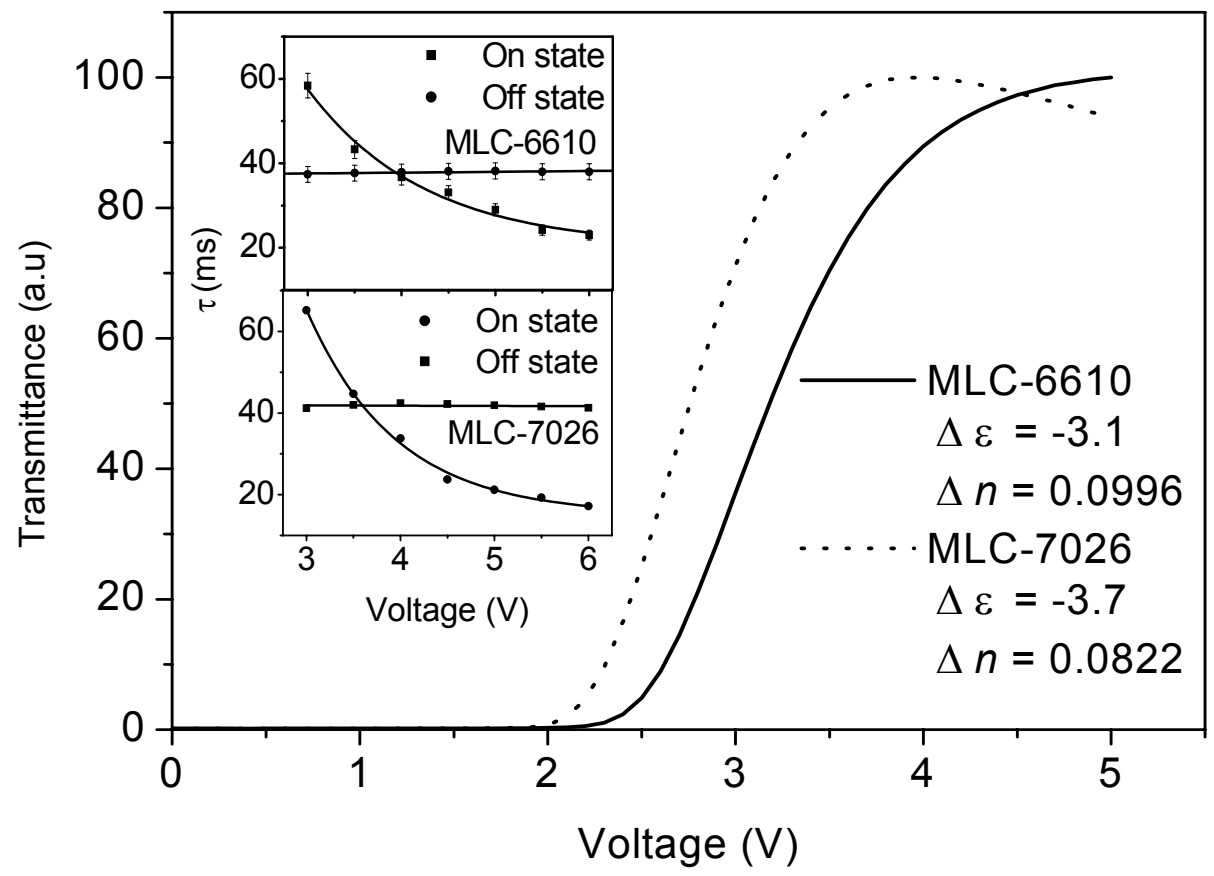

Figure 5.10: Comparison of transmission-voltage curve at normal incidence for a twisted vertically aligned four-domain liquid crystal display with two different liquid crystals.

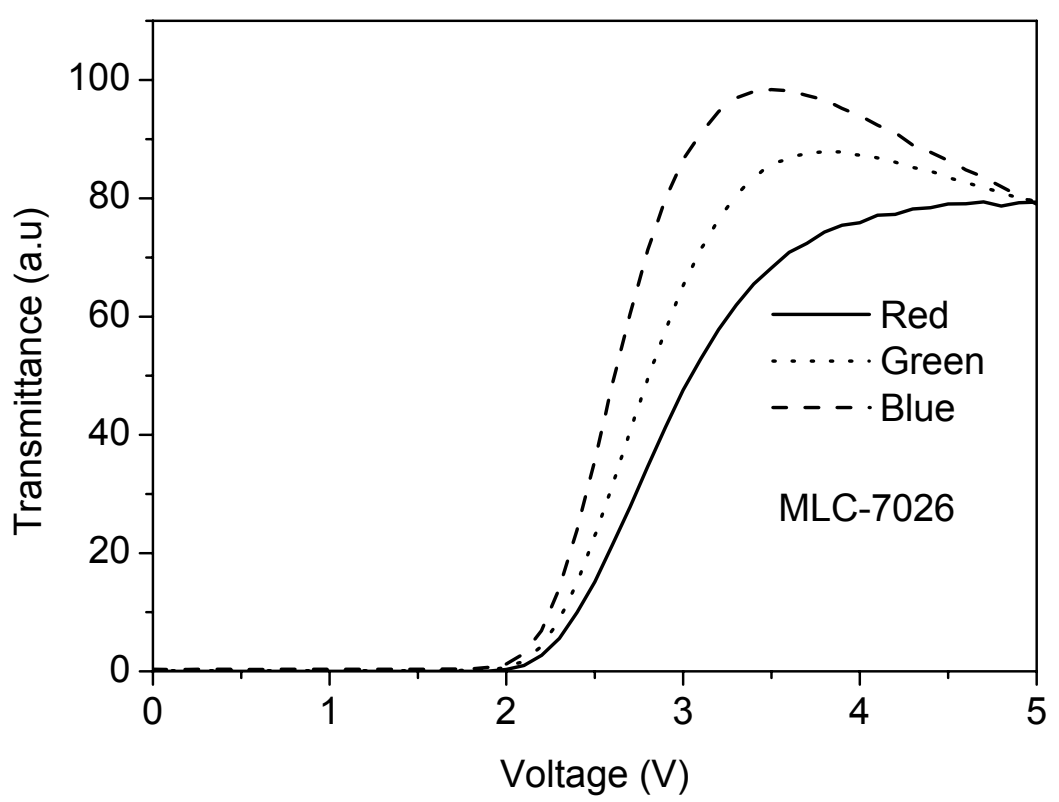

Figure 5.11: Transmission-voltage curve at normal incidence for a twisted vertically aligned four-domain liquid crystal display at different wavelength with liquid crystal MLC-7026. 
Figure 5.11 shows the dependence of three primary colors red $(650 \mathrm{~nm})$, green $(550 \mathrm{~nm})$ and blue $(450 \mathrm{~nm})$ on the transmission-voltage curve at normal incidence with liquid crystal MLC-7026. As in the case of MLC-6610 the optical characteristics are different from each other; therefore, some corrections for displaying high quality color images are necessary.

\subsubsection{Line scanning: - Intensity profile}

Optical microscopy images were recorded of the TVA cell at different voltages. Figure 5.12 shows the plot of light intensity vs. position of disclination lines formed at different voltages $(0$ to $5 \mathrm{~V})$. A line scan performed as shown in Fig. 5.12 (right part) in the optical microscopy images and the light intensity is extracted using Lab View software.

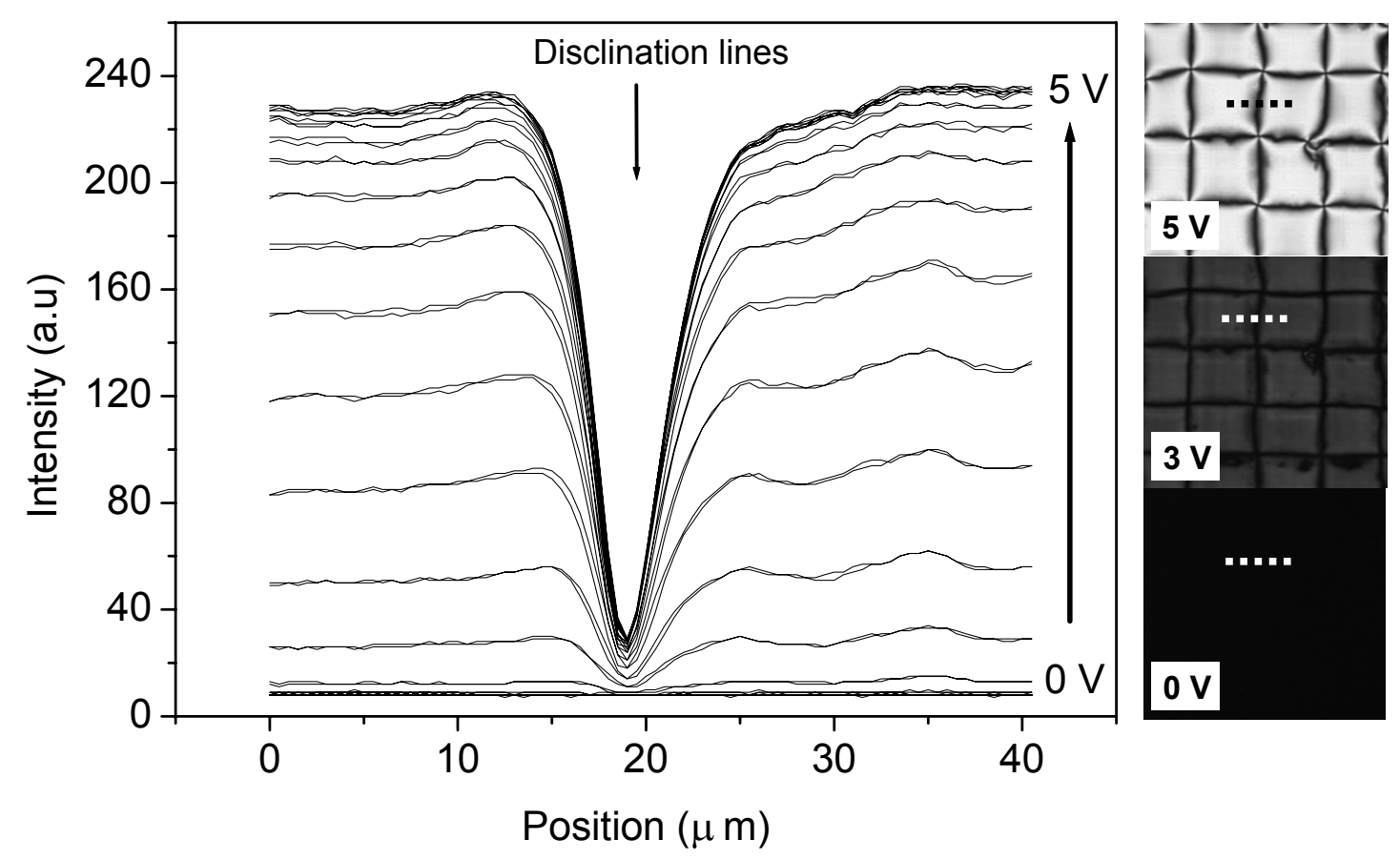

Figure 5.12: Light Intensity profile from the optical microscopy images of the four-domain TVA cell at different voltages $(0 \mathrm{~V}$ to $5 \mathrm{~V}$ with and interval of $0.2 \mathrm{~V})$. The dotted lines in the optical microscopy images indicate the position of line scan. 
The disclination lines are formed due to the difference in twist of liquid crystal molecules in each domain. The formation of disclination lines is unavoidable in multidomain structures due to the orientation of LC directors in different directions. Disclination lines that appear in the bright state only marginally affect the optical contrast of the display.

\subsubsection{Chromaticity measurements}

The white color chromaticity of the four-domain vertically aligned cell was measured with an Eldim conoscope at two different angles using the 1931 color system. Figure 5.13 and 5.14 show the color dispersion at $80^{\circ}$ and $60^{\circ}$ viewing angles for the TVA mode and the FTVA mode respectively. The dark dots in the middle part of the plot represent points of each measurement at different angles.
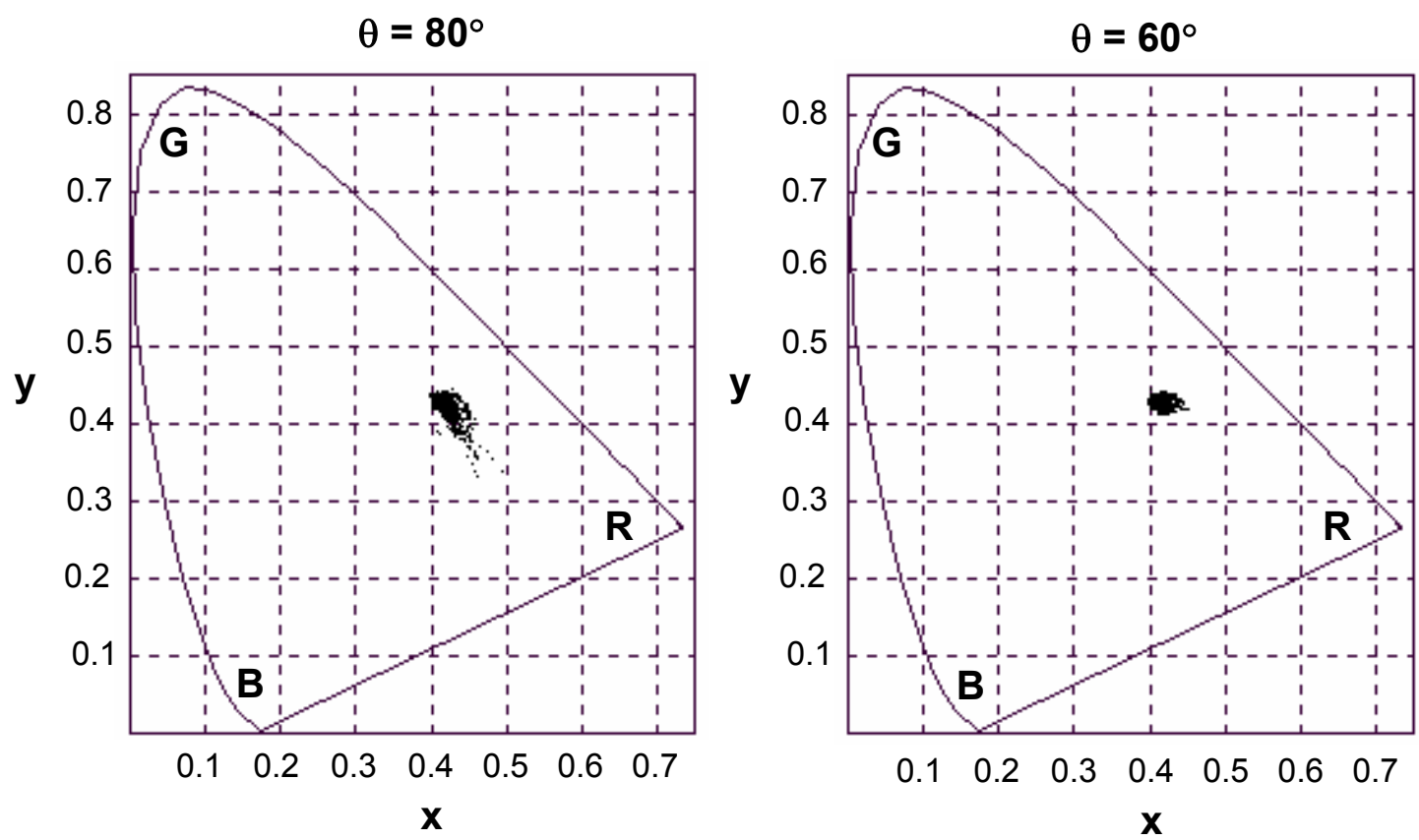

TVA

Figure 5.13: Dependence of white color chromaticity of the four-domain TVA cell at $80^{\circ}$ and $60^{\circ}$ viewing angle. 

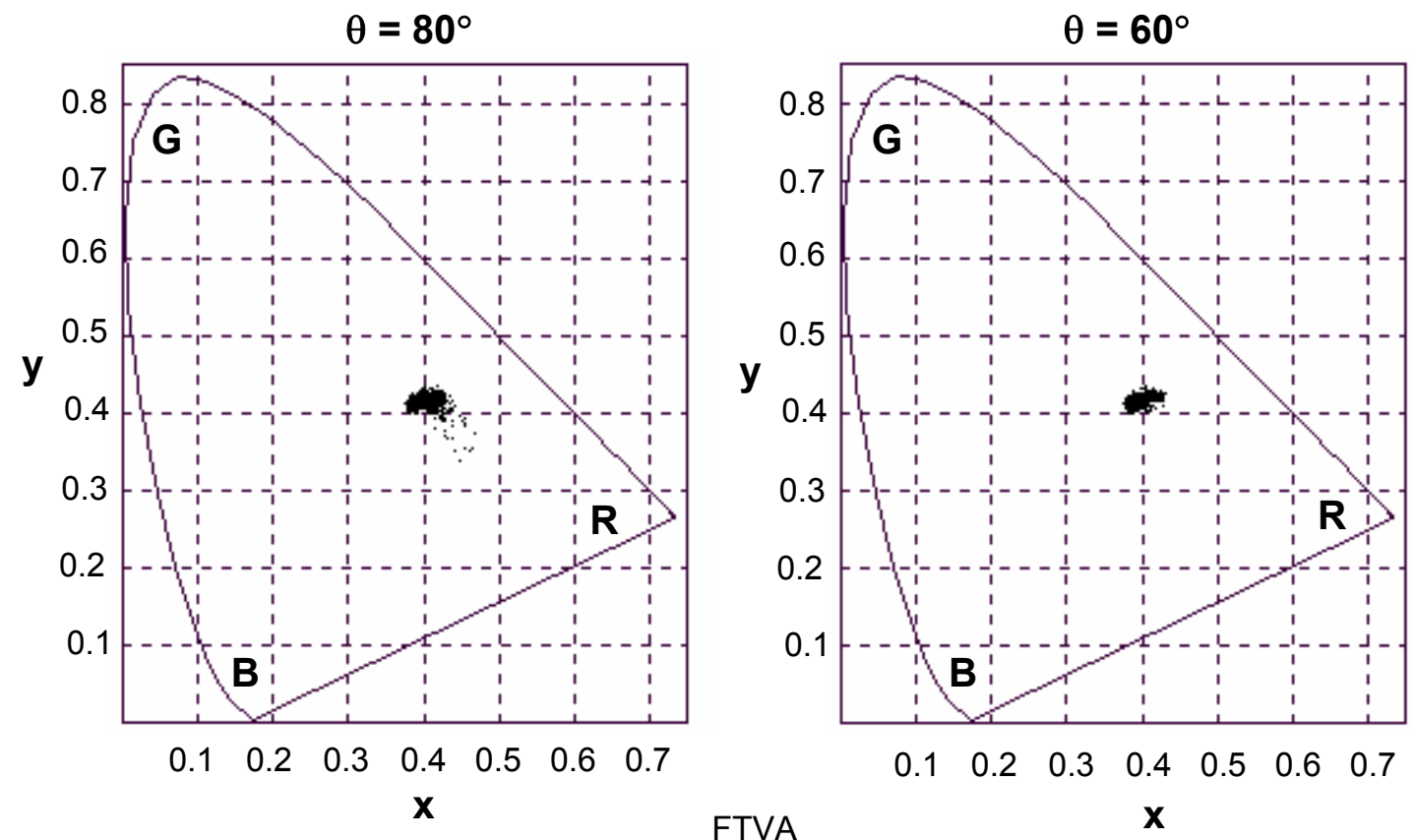

Figure 5.14: Dependence of white color chromaticity of the four-domain FTVA cell at $80^{\circ}$ and $60^{\circ}$ viewing angle.

It can be observed from Fig. 5.13 and Fig. 5.14 that in TVA and FTVA modes the color dispersion is small especially at $60^{\circ}$ indicating reduced dependency of white color chromaticity on azimuthal viewing directions in comparison to a single domain display. This indicates the potential application of this multidomain VA-LCD for full color display applications.

\subsubsection{Optical simulations}

\subsubsection{Viewing angle and contrast ratio}

The viewing angle characteristics of this four-domain structure were calculated using the extended Jones matrix method for five gray levels (see also chapter 4). Figure 5.15 shows the viewing angle characteristics of TVA at three different voltages, 0,4 and $5 \mathrm{~V}$. 
The lines represent the isointensity lines. Symmetric nature of isointensity curve was obtained which are comparable to the experimental data in Fig. 5.5.

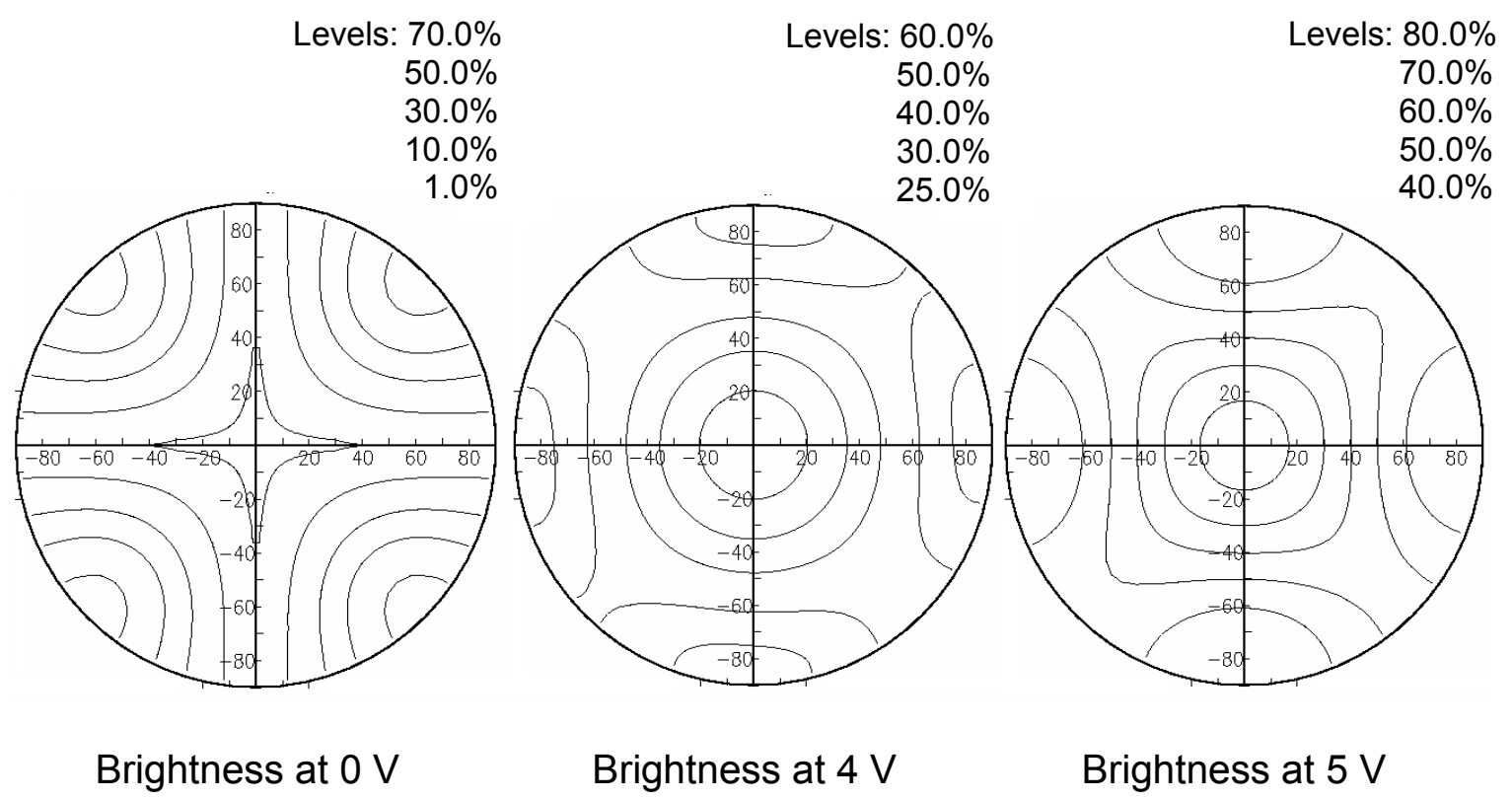

Figure 5.15: The optical simulation results of viewing angle characteristics of the four-domain twisted vertical aligned display at $0 \mathrm{~V}, 4 \mathrm{~V}$ and $5 \mathrm{~V}$.

At zero voltage the TVA cell is in the homeotropic state and light leakage through the polarizers at large angles (polar angle $>45^{\circ}$ and azimuth approximately $45^{\circ}$ ) is expected and observed in the theoretical calculations (Fig. 5.15) and the experimental data (Fig. 5.5).

A comparison is presented between the experimental and simulated contrast ratio measurements at 0 and $5 \mathrm{~V}$ as shown in Fig. 5.16. The shape of the iso-contrast curve is symmetrical in both the vertical and horizontal directions. To improve contrast ratio for higher viewing angle, a combination of this four-domain structure with a negative $c$-plate optical compensation film is necessary as mentioned in Chapter 1. 

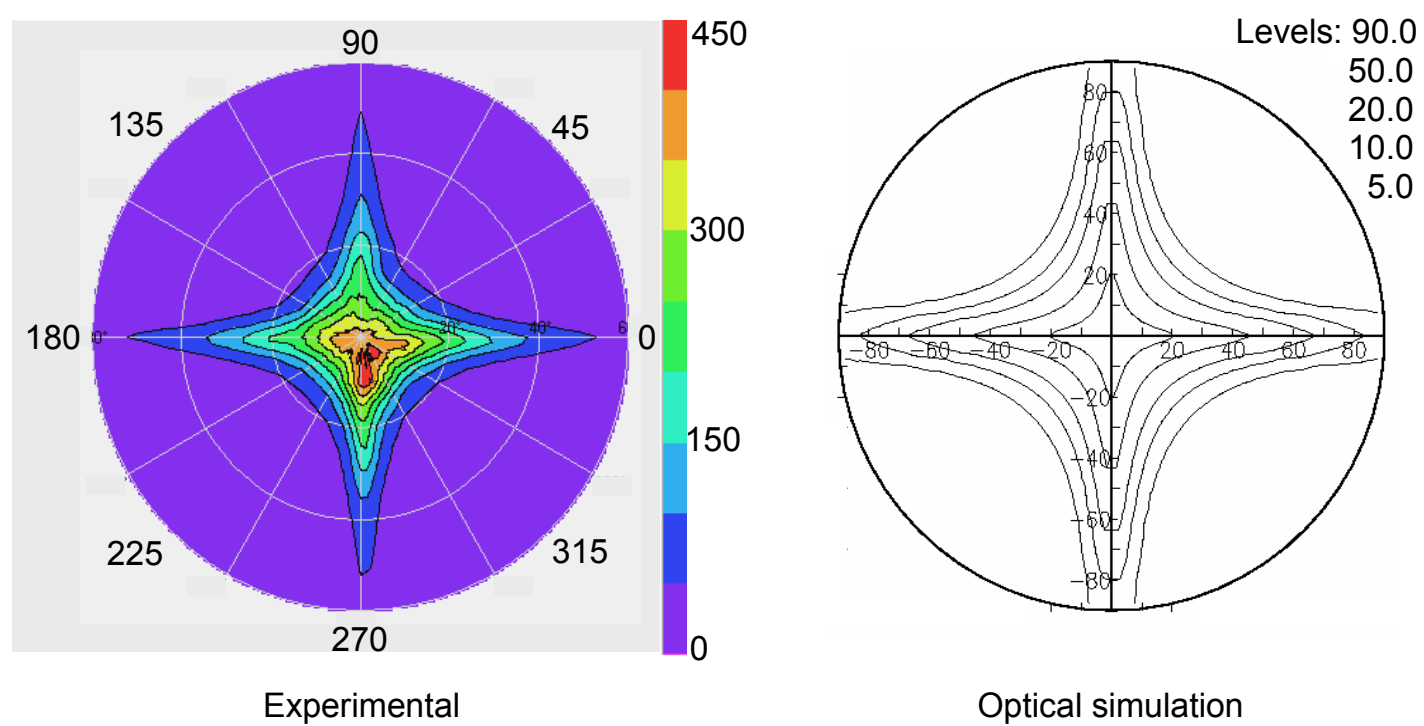

Figure 5.16: Comparison of experimental and theoretical results for the contrast ratio measurement of the four-domain TVA cell.

\subsubsection{Director orientations}

Figure 5.17 and Fig. 5.18 shows the 2D director patterns (vertical cross sections) simulated with the 2 dimMOS program. ${ }^{12,13}$ Table 5.1 list the parameters of TVA cell used in the optical simulations. The figures depict the vertical cross sections of the cell having two sub-pixels. The gray rods represent the directors, where the light end indicates a forward direction and the dark ends indicate a backward direction with respect to the plane of drawing. In this calculation a cross section of a pair of $\mu$-rubbed patterns on the top substrate and a single $\mu$-rubbed pattern on the bottom substrate, which are orthogonal to each other are selected. The left half of the top substrate has a pretilt of $89^{\circ}$ ( $\mu$-rubbed area) with an azimuthal angle of $90^{\circ}$ and the right half has the same pretilt with an azimuthal angle of $270^{\circ}$. The bottom substrate possesses the same pretilt with an azimuthal angle of $180^{\circ}$. 
At zero fields a perfect homeotropic alignment of the directors is observed in the simulation as shown in Fig. 5.17. From the simulations study we observe the orientation of directors in an applied field and the formation of disclination lines. In Figure 5.18 the directors in the two-domains are inclined in opposite directions. In an applied field this results in the formation of disclination line in which the director rotates over $180^{\circ}$, giving rise to dark regions in the transmission as shown on the top part of the figure, which are comparable with the experimental observations in Figure 5.5.

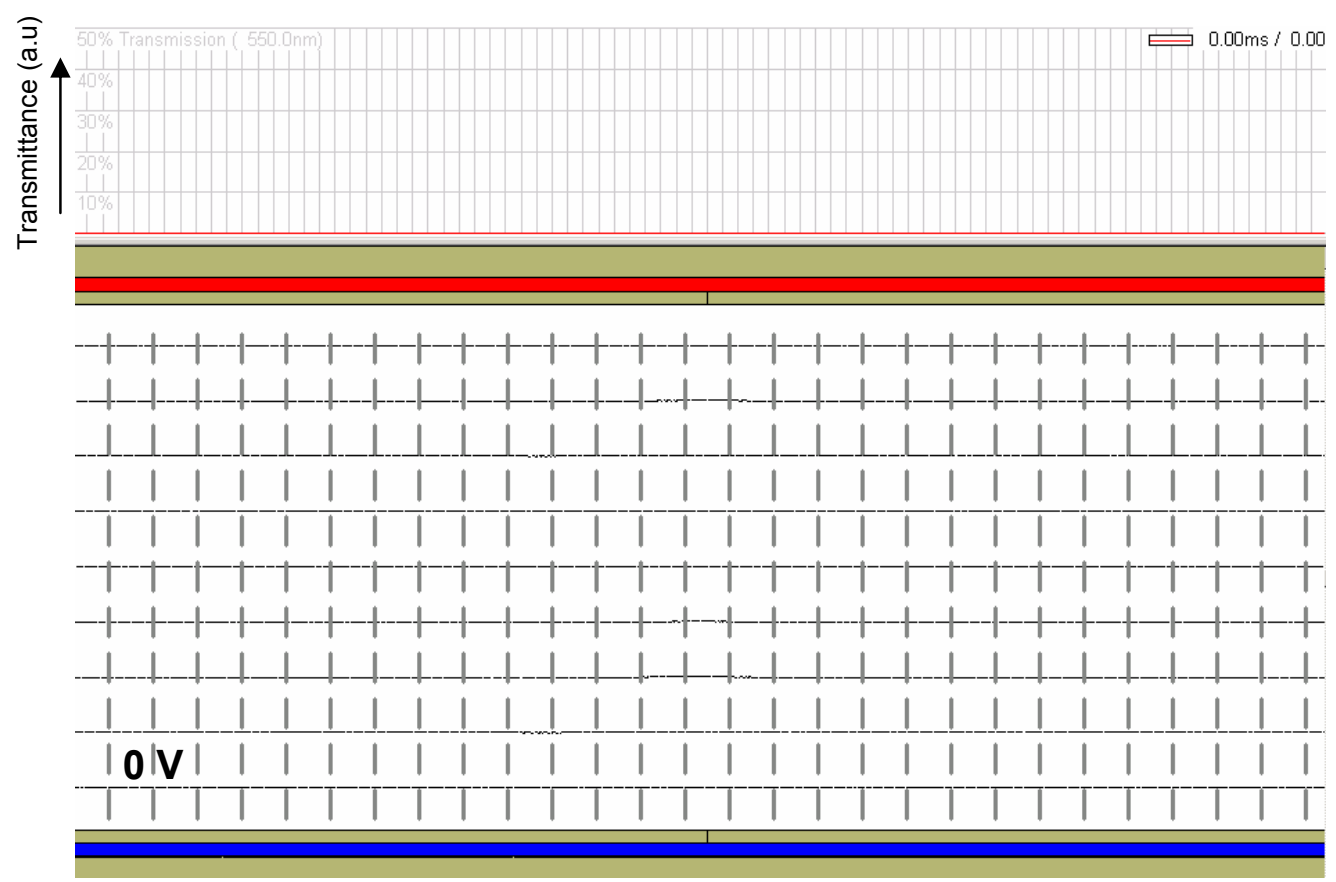

Figure 5.17: Results of 2dimMOS calculations for the director orientation of the four-domain TVA cell at $0 \mathrm{~V}$. Calculated transmittance over the same section is plotted above. 

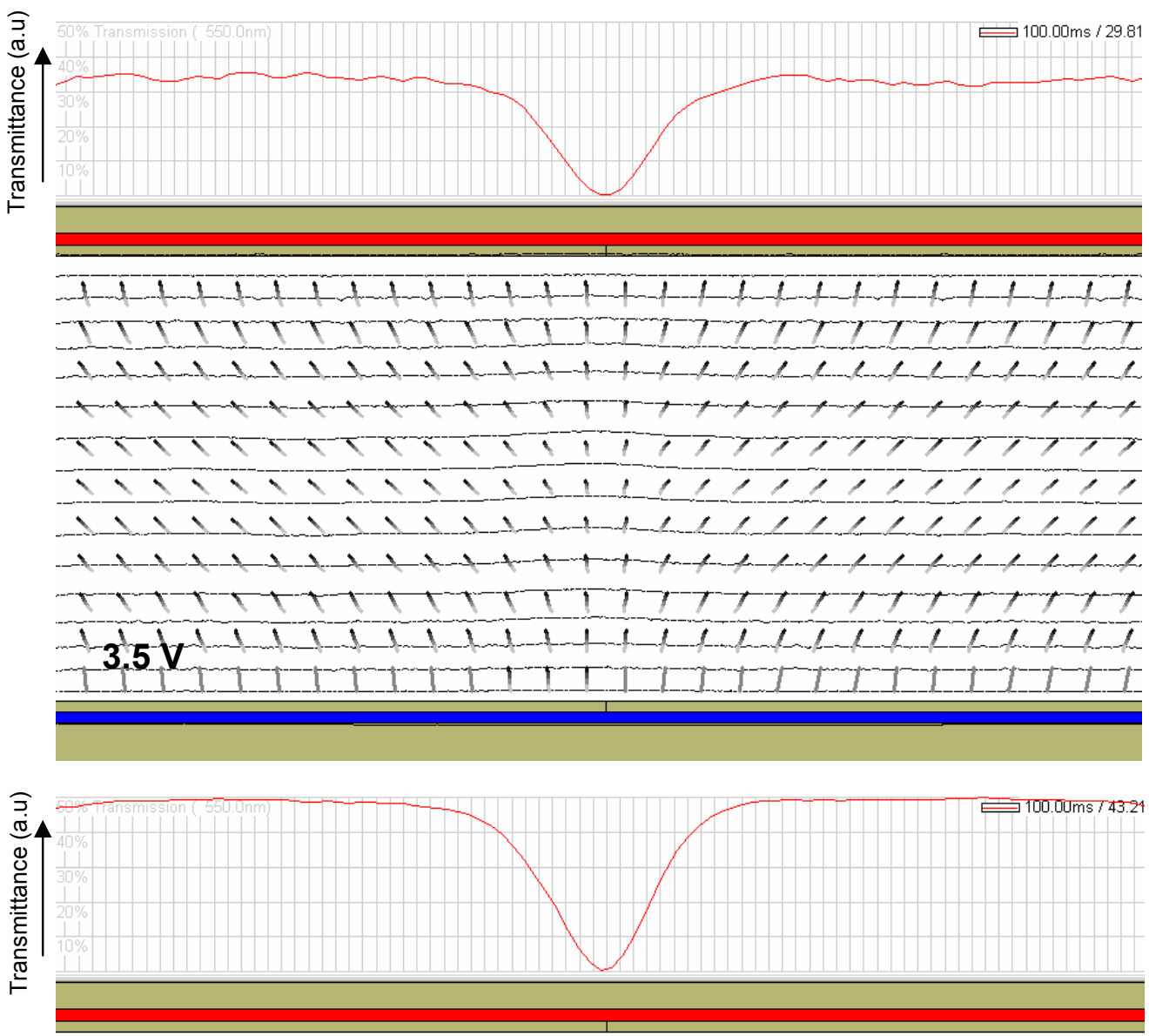

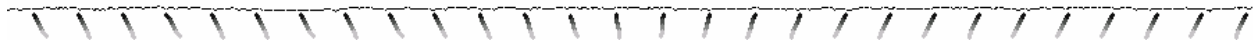
-

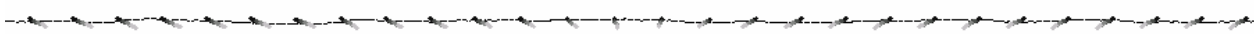
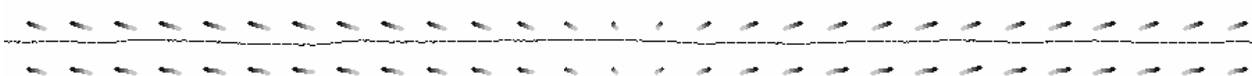

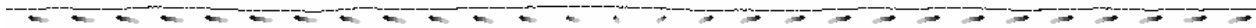

$=2=-2=-1$

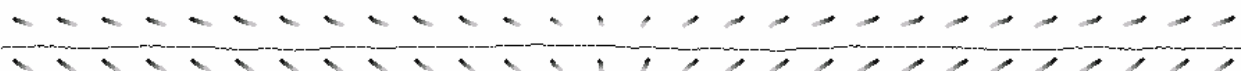

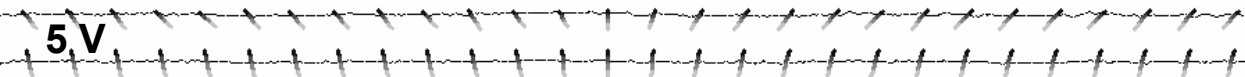

Figure 5.18: Results of 2dimMOS calculations for the director orientation of the four-domain TVA cell at 3.5 and $5 \mathrm{~V}$. Calculated transmittance over the same section is plotted above. 
Table 5.1: The parameters of TVA cells used in the optical simulations. (The material parameters of MLC-6610 are used.)

\begin{tabular}{cll}
\hline \hline & & \\
Dielectric anisotropy & $\varepsilon_{/ /}$ & 3.5 \\
$\left(20^{\circ} \mathrm{C}, 1 \mathrm{kHz}\right)$ & $\varepsilon_{\perp}$ & 6.6 \\
& $\Delta \varepsilon$ & -3.1 \\
Elastic constants $\left(20^{\circ} \mathrm{C}\right)$ & $k_{11}$ & $14.6 \mathrm{pN}$ \\
& $k_{33}$ & $16.5 \mathrm{pN}$ \\
& $k_{33} / k_{11}$ & 1.13 \\
Optical anisotropy & $\Delta n$ & 0.0996 \\
$\left(20^{\circ} \mathrm{C}, 589 \mathrm{~nm}\right)$ & $n_{e}$ & 1.5824 \\
& $n_{o}$ & 1.4828 \\
Cell gap $(\mu \mathrm{m})$ & $d$ & 5.0 \\
Pretilt angle & $\theta_{p}$ & $89^{\circ}$ \\
\hline
\end{tabular}

\subsubsection{Disclination lines}

Figure 5.19 shows the line scans of the optical microscopy image (TVA cell) as well as the simulated intensity profiles at $0 \mathrm{~V}, 3.5 \mathrm{~V}$ and $5 \mathrm{~V}$. For the line scans a straight line was drawn over the image across the disclination lines and the intensity vs. position was plotted. The experimental and optical simulation results are in good agreement. At higher voltage the disclination lines appear due to the difference in the orientation of the directors in each sub-pixel. A slight asymmetry is seen in the curve. The intensity vs. position changes more steeply on the right side of the disclination since there the twist helps to align the directors in the plane of drawing, parallel to one of the polarizer directions (see Fig. 5.18). 

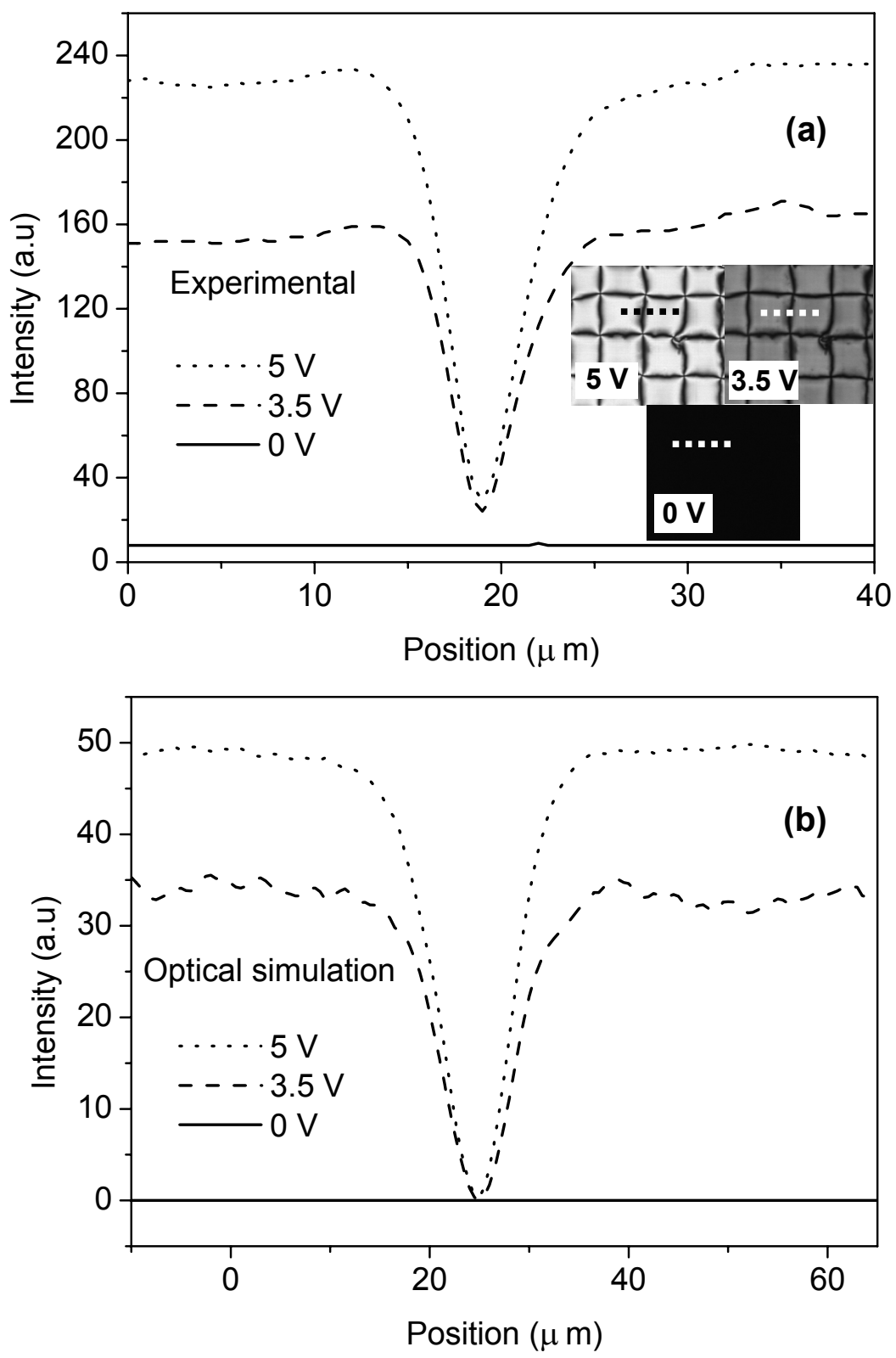

Figure 5.19: Comparison of experimental and calculated intensity profile near the disclination lines in the TVA mode at different voltages. Inset shows the corresponding optical microscopy image, the dotted line shows the area of line scanning. 


\subsubsection{Electro-optical characteristics}

Like in the previous chapters the extended Jones matrix method was used for the calculation of switching characteristics.

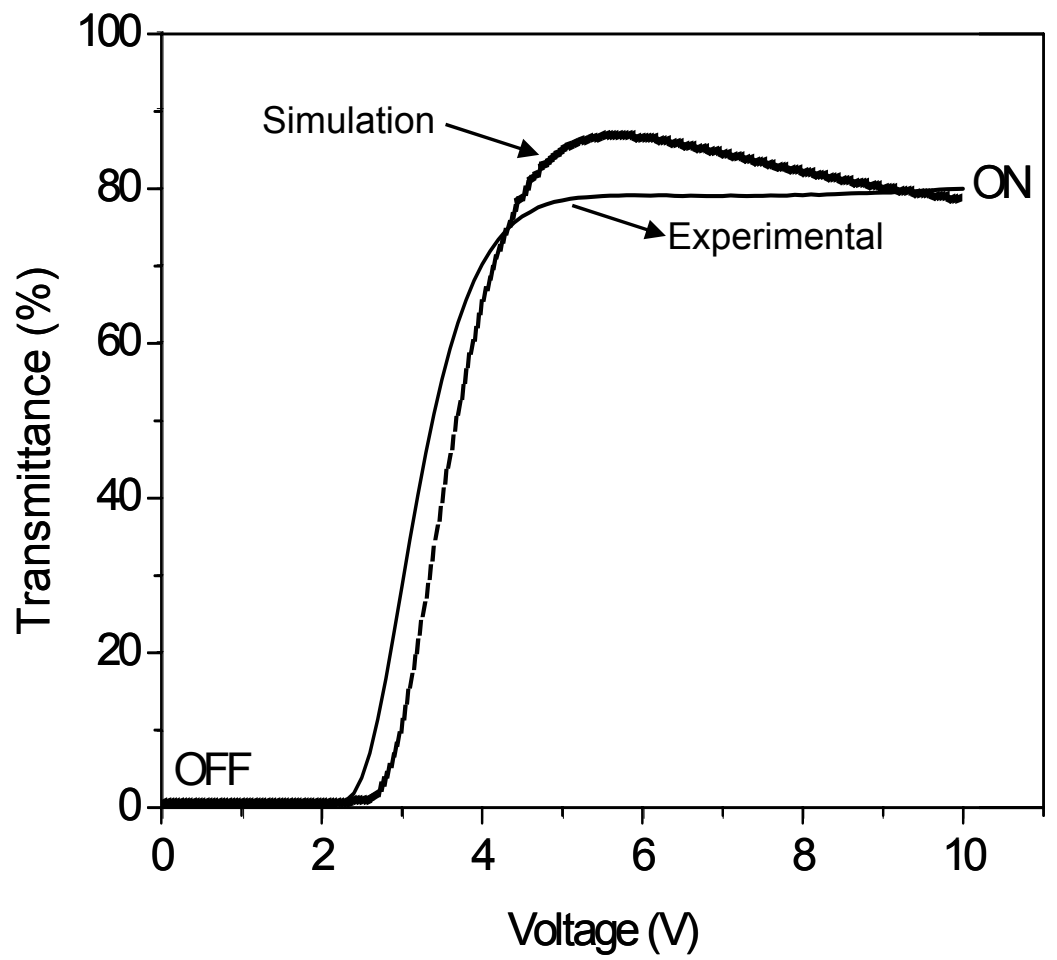

Figure 5.20: Comparison of experimental and simulated transmission-voltage curves at normal incidence of a twisted four-domain vertically aligned cell.

Figure 5.20 shows the comparison of the experimental and simulated transmission-voltage (T-V) curves of a TVA four-domain cell. The slight discrepancies in the simulation may be due to small deviations in the elastic constants $(k)$ and dielectric constant $(\Delta \varepsilon)$, but also due to a weaker anchoring than assumed in the calculation.

The $\mu$-rubbing of this specific polyimide give a small change in pretilt angle with respect to the normal to give vertical alignment. This precise control of pretilt angle is important in multidomain VA displays. This multidomain structure is stable and robust and the switching behavior is comparable with the conventional VA displays. The viewing angle can be further improved by using a suitable optical compensation film. 
The TVA-LCD shows very low color dispersion that opens the possibility to manufacture full color displays. Here the FTVA mode is interested to study the influence of chiral dopant to the twist sense of the sub-pixels. The addition of chiral dopant reduces the contrast of the display due to frustration in the sub-pixel with opposite twist. By changing the ball diameter or by using lower load it is also possible to make small sub-pixels for high resolution applications. In practical display manufacturing process many ball systems (comb-like) can be utilized. This technique is simple and economical as compared to lithographic techniques currently used for multidomain vertical alignment. This $\mu$-rubbing process can be potentially scaled up and used in the production of large area displays in future.

\subsection{Conclusions}

The $\mu$-rubbing of a homeotropic polyimide was investigated and it was found that an extremely high pretilt $\left(89^{\circ}\right)$ was obtained. Consequently, multidomain TVA mode electro-optical cells were constructed and their properties were explored. It was shown that electro-optical cells with a highly symmetrical viewing cone, extremely high contrast, low color dispersion and good switching characteristics were obtained. The experimental and optical simulations are in good agreement. This technique is potentially useful for the industrial manufacture of full color display based on twisted vertically aligned mode. The chiral dopant was added to the LC in the TVA mode to study the twist sense of the sub-pixels that provides an FTVA mode pixel. However, this reduces display properties such as contrast due the presence of wrong twist sense or frustration in the diagonal sub-pixels. Therefore, the TVA mode is recommended in actual display applications.

\section{5 References}

[1] J. Y. Hwang, K. J. Lee, D. S. Seo, T. H. Kim, Jpn. J. Appl. Phys. 42, L672 (2003).

[2] Y. Koike, K. Okamoto, FUJITSU Sci. Tech. J. 35, 221 (1999). 
Four-Domain Twisted Vertically Aligned Liquid Crystal Display Using $\mu$-Rubbing 123

[3] S. C. A. Lien, C. Cai, R. W. Nunes, R. A. John, E. A. Galligan, E. Colgan, J. S. Wilson, Jpn. J. Appl. Phys. 37, L597 (1998).

[4] H. Vithana, D. Johnson, P. Bos, Jpn. J. Appl. Phys. 35, L320 (1996).

[5] J. Chen, P. J. Bos, D. R. Bryant, D. L. Johnson, S. H. Jamal, and J. R. Kelly, Appl. Phys. Lett. 67, 1990 (1995).

[6] B. L. V. Horn, H. H.Winter, Appl. Opti. 40, 2089 (2001).

[7] K. A. Crandall, M. R. Fisch, R. G. Petschek, C. Rosenblatt, Appl. Phys. Lett. 65, 118 (1994).

[8] H. Yoshida, T. Seino, Y. Koike, Jpn. J. Appl. Phys. 36, L1449 (1997).

[9] M. Lu, K. H. Yang, Jpn. J. Appl. Phys. 39, L412 (2000).

[10] L. M. Blinov, V. G. Chigrinov, Electro-optic effects in liquid crystal materials, Springer-Verlag, New York, Chapter 4 (1996).

[11] T. J. Scheffer, J. Nehring, Appl. Phys. Lett. 45, 1021 (1984).

[12] M. E. Becker, H. Wohler, M. Kamm and J. Kreis, Proceedings of SID 96, 1996, 596.

[13] 2dimMOS, www.autronic-melchers.com. 


\section{Chapter 6}

\section{Surface-Induced Pattern Formation in LC Mixtures*}

\subsection{Introduction}

Patterning of substrates on a micron and sub-micron scale is of considerable technological interest in microelectronic circuits, displays, telecommunication technology and digital data storage media. Scientists and engineers are looking for simple low cost techniques for the reliable replication of both nanometer and micrometer sized patterns. Steiner et al, proposed several patterning routes in which dewetting and phase separation of blends is exploited to fabricate patterns on the micrometer scale. ${ }^{1-3}$ The processes consist of demixing and/or phase separation of a binary polymer blend thin films during spin coating on a patterned gold surface. The gold surface is patterned with self assembled monolayers consisting of both hydrophilic and hydrophobic regions. A polystyrene/polyvinyl pyridine (PS/PVP) blend was used. The structure of the patterned gold substrate was mimicked in the polymer morphology due to migration of the polar PVP to the hydrophilic regions and the migration of PS to the hydrophobic regions. Whitesides et al reported the phase separation of ultra thin polymer blend films of polystyrene and polybutadiene on micro-contact printed alkane thiol patterns with hydrophobic and hydrophilic end groups. ${ }^{4}$ It was found that this controlled phase separation was strongly dependent on the composition of the polymer mixtures and on the physical properties of the patterned self assembled monolayers.

In this chapter, a new method is presented for the spatially controlled phase separation of mixtures containing a liquid crystal (LC) and a non-liquid crystalline

*This chapter is partly reproduced from: S. Varghese, S. Narayanankutty, C. W. M. Bastiaansen, D. J. Broer, Proceedings, Advances in Polymer Technology, Cochin 110, (2002). 
material. For the non-liquid crystalline material polyethylene glycol was selected. The molecular weight was chosen to be moderate to enhance solubility in the LC at elevated temperature but to phase separate upon cooling. The polyethylene glycol is assumed to be more polar than the LC material. A pattern is created on a substrate with well defined regions of a high and low surface tension and surface polarity. Spatially controlled phase separation of the liquid crystal containing mixtures opens the possibility to fabricate active patterns in which the liquid crystals can be switched electrically. Figure 6.1 shows a model substrate having differences in surface polarity and the pattern formed with well defined regions consisting of liquid crystals and polymers. By switching the liquid crystal in an electrical field the refractive index difference between the hydrophobic and the hydrophilic areas can be modulated and the device can be switched. These kinds of patterns are of potential interest in, for instance, switchable gratings for telecommunication applications. 5,6

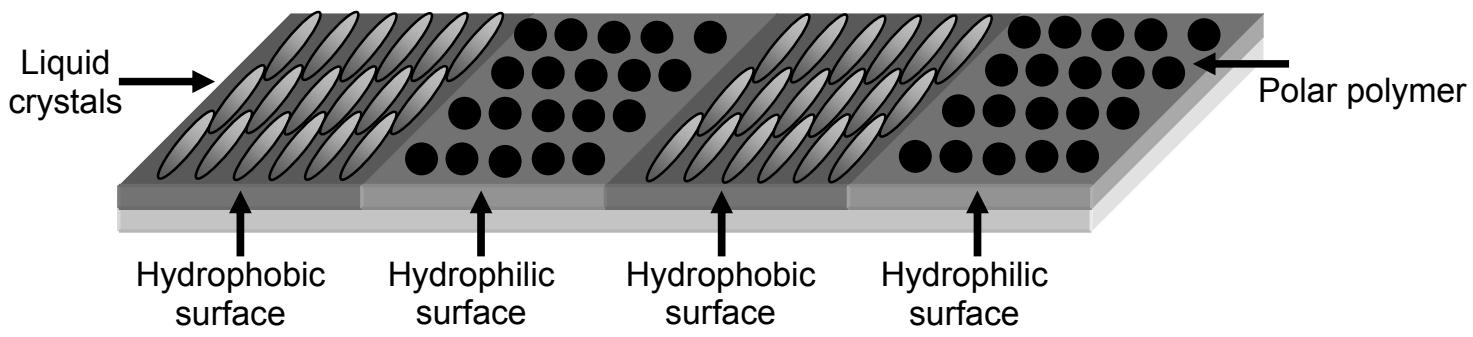

Figure 6.1: Model system for the phase separation of liquid crystal mixtures.

\subsection{Experimental}

\subsubsection{Materials}

Indium tin oxide (ITO) coated glass $(25 \mathrm{~mm} \times 25 \mathrm{~mm})$ was obtained from Merck. The ITO coated glass substrate was thoroughly washed with a soap solution, deionised water and ethanol and dried. Homeotropic polyimide (AL75114) and planar polyimide (A11051) precursor for the orientation layers were purchased from JSR electronics. The polyimide 
precursor was spin coated ( $5 \mathrm{~s}$ at $1000 \mathrm{rpm}$ followed by $40 \mathrm{~s}$ at $5000 \mathrm{rpm}$ ) on the ITO side of the glass substrate. It is then preheated to $100{ }^{\circ} \mathrm{C}$ for $10 \mathrm{~min}$ and imidized at $180{ }^{\circ} \mathrm{C}$ under vacuum for 90 minutes to get a transparent film with a thickness of $\sim 100$ $\mathrm{nm}$. For the planar alignment the polyimide substrate is rubbed unidirectionally using a velvet cloth. Polytetrafluoroethylene (PTFE) rods were purchased from DCA instruments. The liquid crystals E7 was obtained from Merck, Germany. O-[2-(3mercaptopropionylamino) ethyl]-O'-methyl-polyethyleneglycol-5000 (PEG-SH) was purchased from Fluka and polyethylene glycol (PEG-1000) were obtained from Aldrich. Gold was sputter coated with an Emitech, K575X instrument at $60 \mathrm{~mA}$ for $60 \mathrm{~s}$.

\subsubsection{Friction deposition of PTFE}

PTFE was deposited on substrates using a Tribotrack from DACA instruments. This instrument is equipped with a moving stage with varying speed having heating elements inside. The upper portion of the instrument is a stationary arm with a cylindrical foot with heating coils. The top and bottom stage has independent heating control. On this cylindrical foot PTFE rods can be firmly fixed and on the top of the cylindrical foot different loads can be applied as shown in Fig. 6.2. The cylindrical PTFE rod was patterned (width: $0.5 \mathrm{~mm}$ ) and it is fixed to the stationary arm of the Tribotrack. The deposition of PTFE was carried out at $330{ }^{\circ} \mathrm{C}$ and at a speed of $0.5 \mathrm{~mm} / \mathrm{s}$ with a suitable load in the range of 20 to $50 \mathrm{~N}$. From literature, it is known that the PTFE film formed is close to monomolecular in thickness, highly oriented and which depends on temperature, load and speed of film deposition. ${ }^{7-9}$

\subsubsection{Cell construction}

Liquid crystal cells were constructed with two substrates placed orthogonal to each other with respect to PTFE film deposition direction. The substrates were secured with UV curable glue (Norland UV sealant 91) along the edges. The cell gap was controlled with $18 \mu \mathrm{m}$ spacers. The cells were filled with liquid crystal by capillary action at $80{ }^{\circ} \mathrm{C}$, which is $\sim 20^{\circ} \mathrm{C}$ above the nematic-isotropic transition temperature of the liquid crystal. 


\subsubsection{Characterization}

Surface morphology of the PTFE layer was investigated by atomic force microscopy (AFM), Nanoscope Dimension 5000 AFM with a nanoscope III controller (Digital instruments, Santa Barbara, CA) with a scan rate of $1 \mathrm{~Hz}$. Cell gap measurements were carried out using Shimadzu UV-3102 PC UV-VIS-NIR scanning spectrophotometer. Advancing contact angle measurements were performed using Kruss drop shape analysis system, DSA. The angle between the water droplet and the surface was measured through a microscope objective. Electro-optical measurements were performed on a DMS 703 display measuring system (Autronic-Melchers Gmbh) with an AC square wave to drive the display cells $(1 \mathrm{kHz})$. The alignment of liquid crystals was studied using polarized light microscopy, Zeiss LM Axioplan. The pretilt angle was measured directly using an Autronic TBA 107 instrument (Autronic-Melchers Gmbh) that uses the crystal rotation method. The calorimetric analysis was performed using of a Perkin Elmer Pyris 1 DSC with a heating rate of $10^{\circ} / \mathrm{min}$. To study the phase behavior using optical microcopy the cell was placed in a microscope on a Linkam THMS 600 hot-stage. The cell was heated and cooled at a rate of $10^{\circ} / \mathrm{min}$, the temperature during the phase transitions are measured.

\subsection{Results and discussion}

\subsubsection{Friction deposition of PTFE on glass substrates}

In this study PTFE film was selected as the hydrophobic surface, which can simultaneously give planar alignment of LCs. ${ }^{7}$ Figure 6.2 shows the experimental setup used in the friction deposition process. A PTFE rod was provided with a surface relief profile by micro-machining before the friction deposition process. A pattern was generated with a pitch and depth of $0.5 \mathrm{~mm}$. In an initial set of experiments, the surface morphology of PTFE film, the pretilt angle and the switching behavior were studied. 


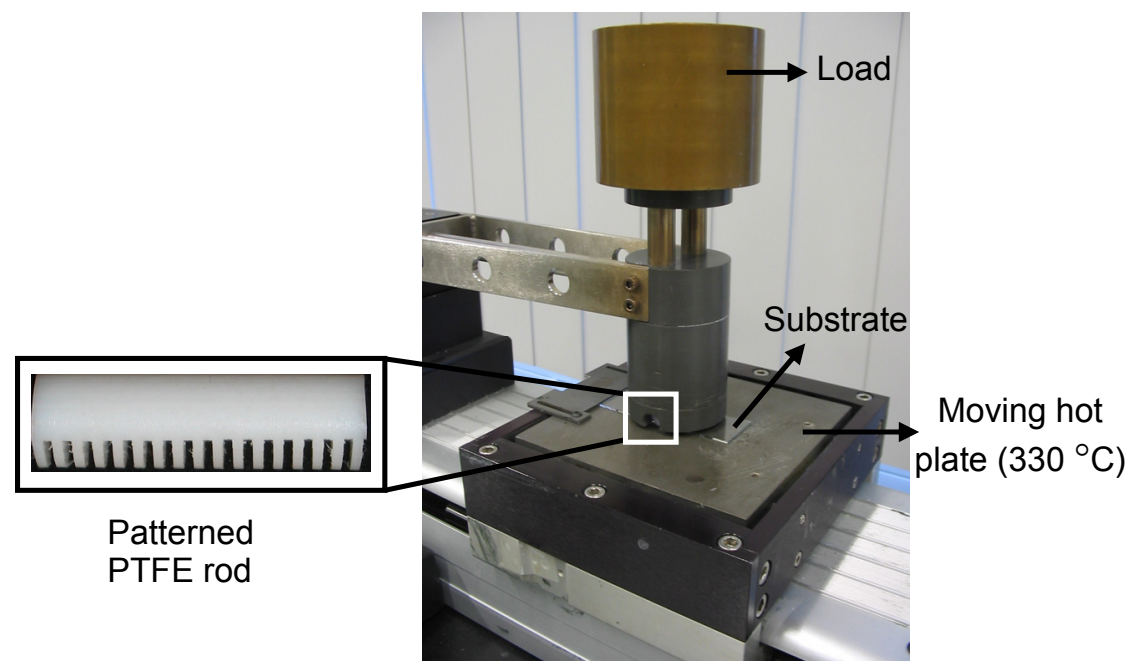

Figure 6.2: Experimental setup used for the friction deposition of PTFE film.

Figure 6.3 shows an AFM image of a friction deposited PTFE film. From the AFM image it is obvious that the friction deposited PTFE layer has a highly fibrillar texture which indicates that the PTFE macromolecules are highly aligned in the deposition direction. ${ }^{8}$ The thickness of the PTFE film ranges from $60-70 \mathrm{~nm}$ which was measured from the height profile after scratching the deposited PTFE film. The film thickness of the PTFE layer depends on the deposition temperature, the load and the deposition speed. A cross section of the height profile of the friction deposited film is also shown in Fig.6.3. Irregular and highly anisotropic grooves are formed during the friction deposition process. In the contact angle measurements the water droplet exhibits an anisotropic shape with the long axis towards the deposition direction of the PTFE with a contact angle of $96^{\circ}$. 


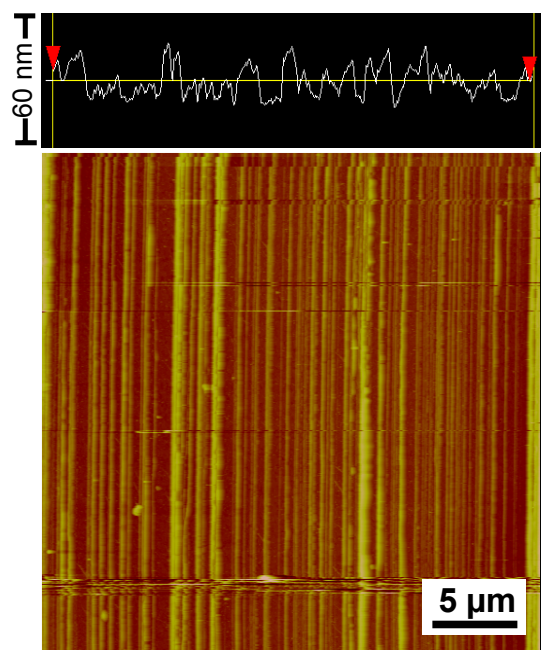

Figure 6.3: Atomic force micrograph of a thin layer of PTFE produced by friction deposition. The thickness of the PTFE layer is 60-70 nm, the height profile is also shown at the top.

\subsubsection{Pretilt angle measurements}

The pretilt angle of LCs on PTFE was measured using the crystal rotation method. For the measurements, LC cells were constructed with PTFE films produced at different loads in cells with uniform cell gap. The pretilt angle was measured by placing the cell at $45^{\circ}$ to the rotation axis of the TBA 107 instrument, to keep the midplane director perpendicular to the rotation axis. Figure 6.4 shows the variation of pretilt angle with applied load during the friction deposition process. It was observed that the pretilt angle decreases with an increasing load. This is related to the change in surface roughness; the surface roughness decreases with increasing load resulting in a smooth surface with low pretilt angle. The deposition of PTFE can be rather inhomogeneous and elongated areas where PTFE is absent can occur especially at low loads. These bare glass areas between the PTFE also affect the pretilt angle of the LC molecules giving rise to an increased value of pretilt angle at low loads. A good quality PTFE film is usually produced at higher deposition loads which also results in a low surface pretilt angle. ${ }^{7-13}$ 


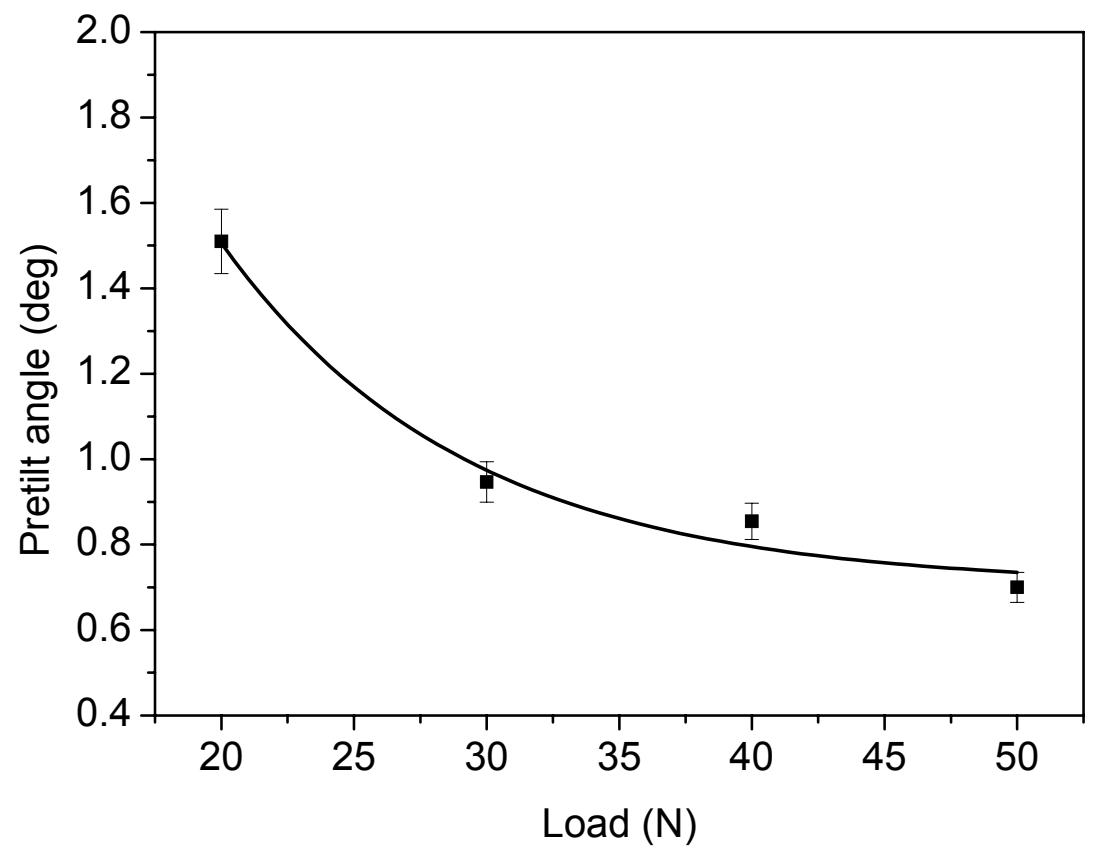

Figure 6.4: Influence of applied load on pretilt angle during the friction deposition process. The PTFE was friction deposited with a speed of $0.5 \mathrm{~mm} / \mathrm{sec}$ at a temperature of $330^{\circ} \mathrm{C}$.

\subsubsection{Electro-optical characterization}

The friction deposition was carried out at different loads at constant temperature $\left(330^{\circ} \mathrm{C}\right)$ on a single substrate. Cells were constructed with two identical substrates and the electro-optical characteristics of the twisted nematic area were investigated. Figure 6.5 shows the transmission-voltage curve of the TN area formed by the PTFE layers. It is obvious from the graph that there is only subtle difference in the transmission characteristics. This subtle shift to higher voltage at higher load is predominantly due to the small differences in the pretilt angle. 


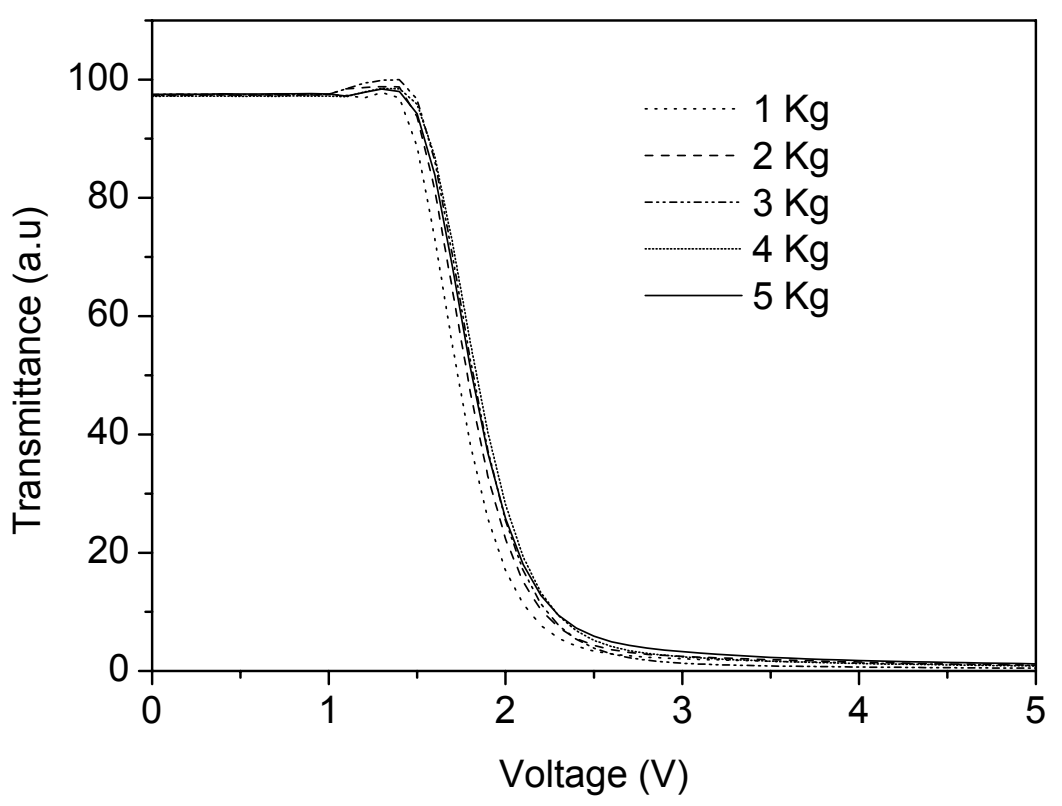

Figure 6.5: Comparison of the electro-optical characteristics of TN cell created by the friction deposition of PTFE at different loads. PTFE films were friction deposited at a temperature of $330^{\circ} \mathrm{C}$

\subsubsection{Alignment of E7 on patterned bi-polymer surfaces}

\subsubsection{PTFE- Homeotropic polyimide}

In a second set of experiments, homeotropic polyimide with a contact angle of $50^{\circ}$ was spin coated onto a glass/ITO substrate. Subsequently, a PTFE pattern was friction deposited onto the PI surface. The excellent thermal stability of polyimide layers enables the deposition process at high temperature $\left(330{ }^{\circ} \mathrm{C}\right)$. PTFE was friction deposited on homeotropic polyimide and liquid crystal cells were constructed with the two substrates having the PTFE film deposition direction orthogonal to each other. Figure 6.6 depicts optical micrographs of a cell filled with liquid crystal E7 and viewed between polarizers. The cells were heated to the isotropic temperature of the LC and subsequently cooled down to establish an equilibrium morphology. In the cell, areas with different types of alignments are observed. The PTFE/PTFE regions posses a twisted nematic orientation, 
the polyimide/polyimide regions have a homeotropic orientation and the polyimide/PTFE areas show splay configuration as depicted in Fig. 6.6.

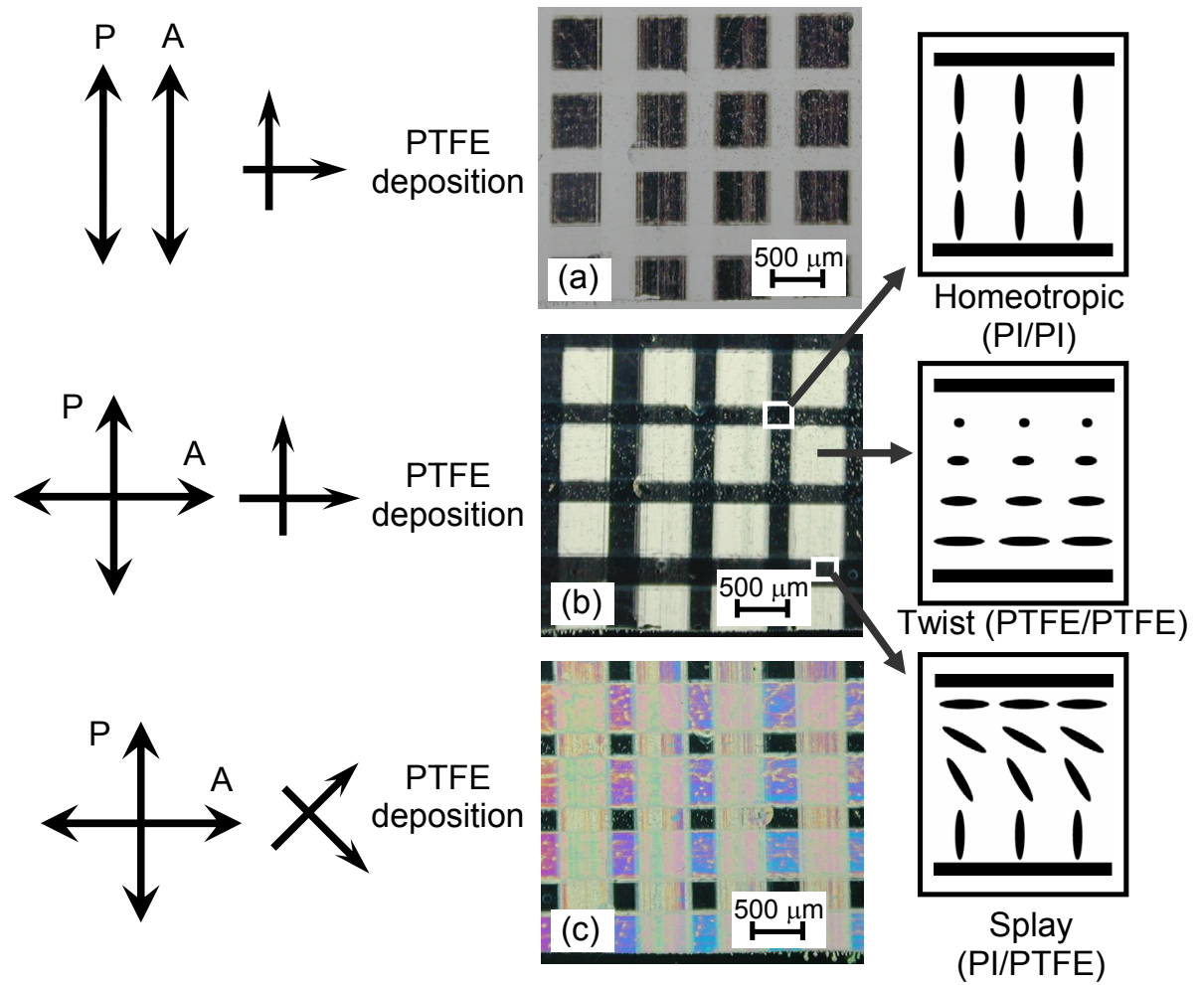

Figure 6.6: Polarized optical micrograph showing the alignment of liquid crystal E7 on patterned homeotropic polyimide with PTFE substrate. (a) The cell viewed between crossed polarizer (b) Parallel polarizer (c) Cell rotated by $45^{\circ}$ between crossed polarizers.

\subsubsection{PTFE- Planar polyimide}

In a third set of experiments alignment layers with larger difference in contact angle were selected. PTFE with a contact angle of $96^{\circ}$ (with water) and a rubbed planar polyimide with a contact angle of $35^{\circ}$ were used. The deposition of the PTFE onto the rubbed PI was carried out at a temperature of $330{ }^{\circ} \mathrm{C}$, at a load of $2.5 \mathrm{Kg}$. In the case of planar polyimide, patterned friction deposition was performed perpendicular to the rubbing direction. Liquid crystal cells were constructed based on two patterned substrates with 
their polyimide alignment directions orthogonal to each other. Figure 6.7 shows the polarized optical micrographs of liquid crystal cells constructed with patterned polyimide/PTFE alignment layers. In the cell, different configurations are observed. A twisted nematic configuration is observed where the polyimide/polyimide or the PTFE/PTFE alignment layers are mutually perpendicular. A planar alignment is observed in areas where there is rubbed polyimide on one side and friction deposited PTFE on the other side.

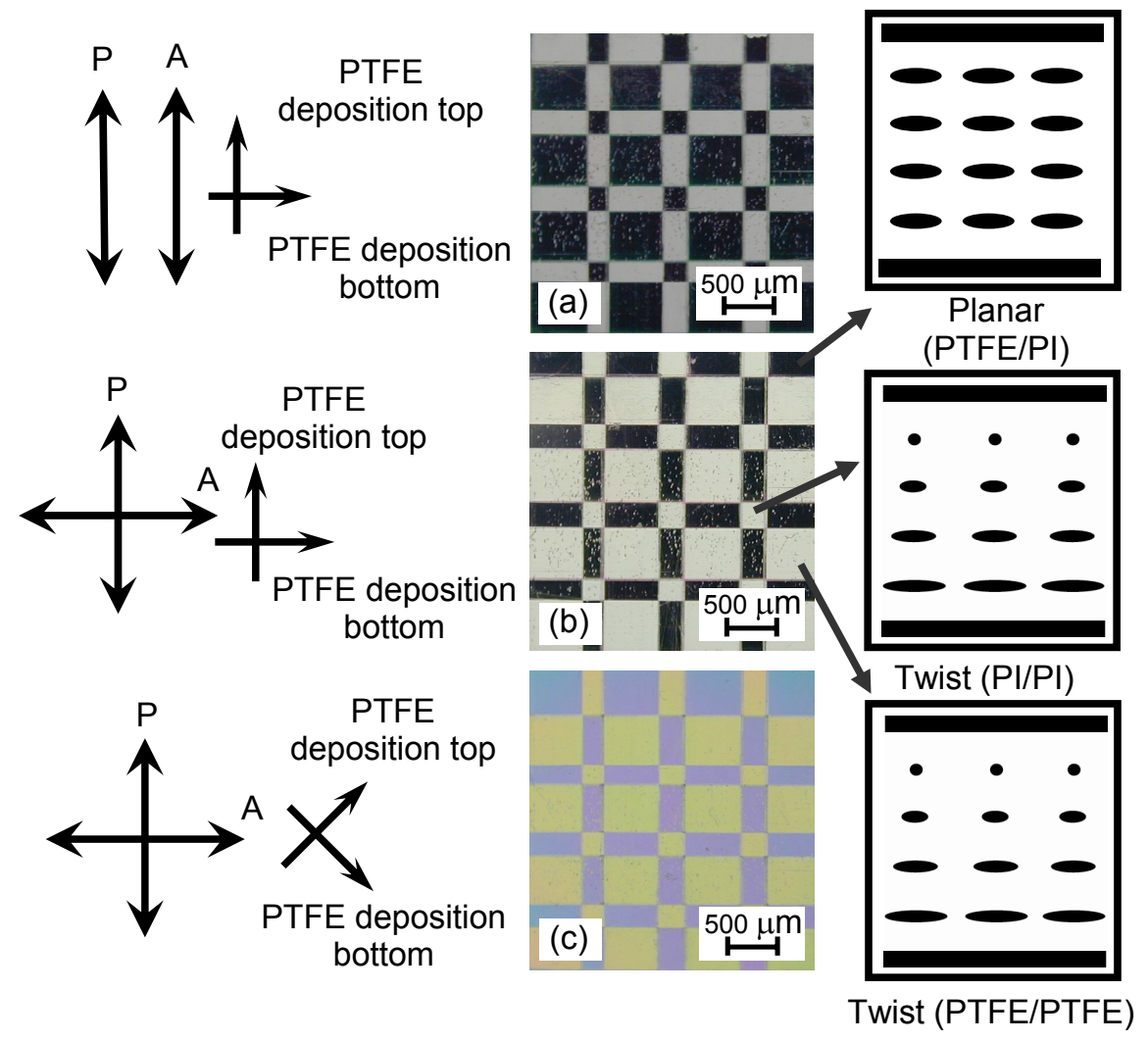

Figure 6.7: Polarized optical micrograph showing the alignment of liquid crystal E7 on patterned planar polyimide with PTFE. (a) The cell viewed between parallel polarizer (b) Crossed polarizer (c) Cell rotated by $45^{\circ}$ between crossed polarizers.

\subsubsection{Electro-optical characterization}

The electro-optical characteristics of the TN areas formed by PTFE on homeotropic polyimide (see Section 6.3.2.1) were also investigated as shown in Fig.6.8. A typical 
switching curve like that of conventionally rubbed polyimide is observed. The threshold voltage is found to be less than for a conventionally rubbed polyimide. This is attributed to the presence of homeotropic polyimide surface between the PTFE stripes, which increases the average pretilt angle giving rise to a lower threshold in the T-V curve.

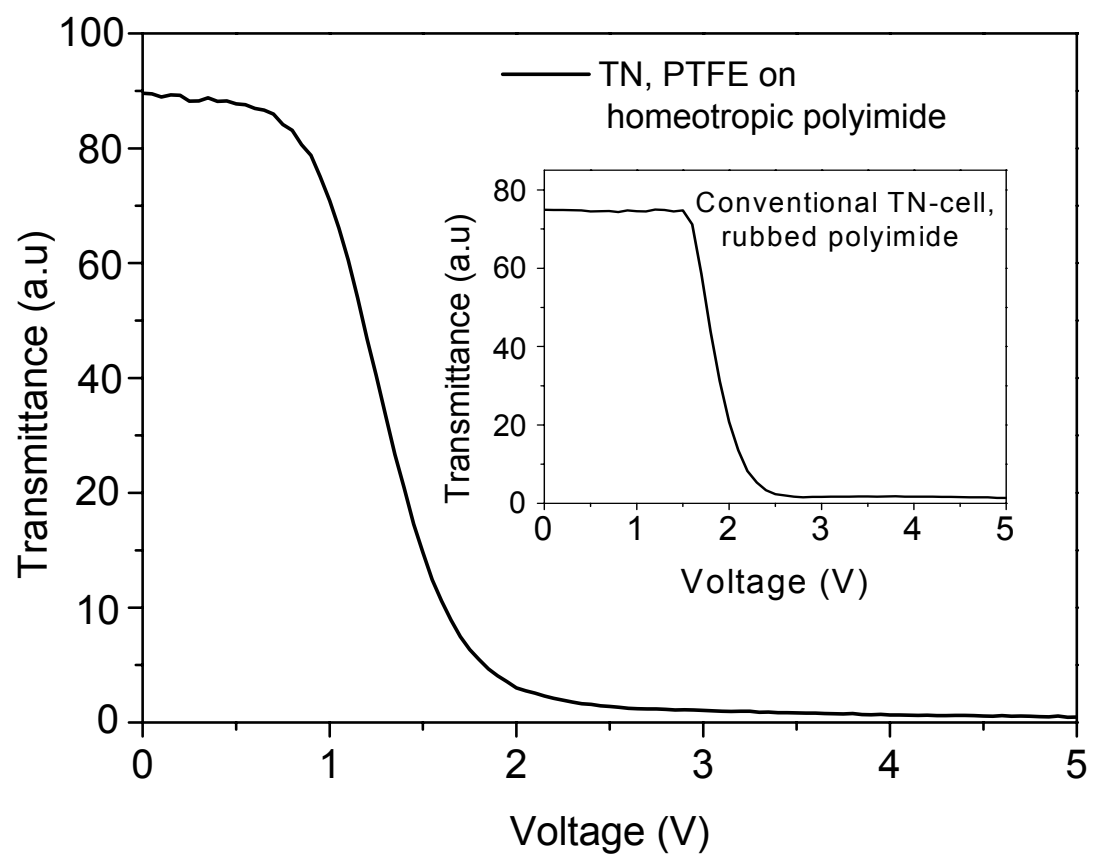

Figure 6.8: Switching characteristics of PTFE orientation layer (TN) on homeotropic polyimide. Inset, $T-V$ curve of conventional TN cell from rubbed polyimide.

The electro-optical characteristics of TN area from the friction deposited PTFE on rubbed polyimide (see Section 6.3.2.2) were also investigated. Figure 6.9 shows the transmission-voltage curve of TN area based on PTFE and polyimide alignment layers. The switching curve of polyimide and PTFE alignment layers are almost identical. It indicates that the TN areas originating from both the polyimide and the PTFE orientation layer switch at almost identical voltages. It also indicates that the possible differences in anchoring energy between the PTFE and the polyimide are of less influence on the switching voltage that strengthen the hypothesis that in the case of the stripes PTFE on homeotropic polyimide the low switching voltages are caused by the homeotropic boundaries along the planar LC stripes. 


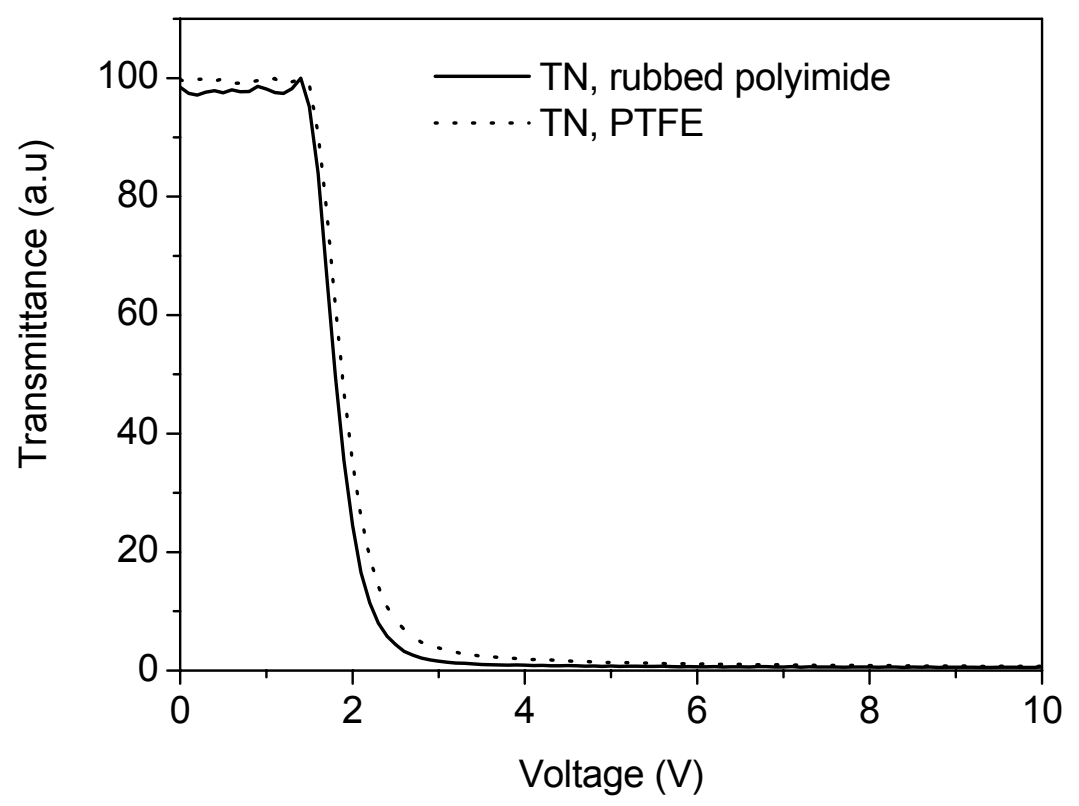

Figure 6.9: Change of transmittance with applied voltage of a conventional TN cell with rubbed polyimide alignment layers and a TN cell with PTFE alignment layer.

\subsubsection{Phase diagram of LC mixtures (PEG-1000/E7)}

To study the phase behavior of LC mixtures, polyethylene glycol (PEG-1000) having a molecular weight of 1000 was selected as a polar component and E7 as the liquid crystal part. The phase transitions of the PEG-1000/E7 system were determined via DSC experiments and optical microscopy with a hot stage during cooling and plotted as a function of the composition as shown in Fig. 6.10. In the phase diagram three different areas are visible, viz. A, B and C. In the A phase, all compositions of PEG-1000/E7 are isotropic and the mixture forms a homogeneous solution. Upon cooling at concentration PEG-1000 $\leq 60 \mathrm{w}-\%$ the homogenous blend phase separates in an E7 rich phase and a PEG-1000 rich phase. The E7 rich phase is liquid crystalline and liquid crystal order is formed upon passing the transition from A to B. It is remarkable that the transition temperature from $\mathrm{A}$ to $\mathrm{B}$ is almost concentration independent up to around $40 \mathrm{w}-\%$ PEG1000 and close to the transition temperature of pure E7, indicating that the phase 
separated E7 contains only minor amounts of the dissolved PEG-1000. At PEG-10000 concentrations somewhat higher than $40 \mathrm{w}-\%$ the isotropic to nematic transition temperature rapidly drops off. The melting temperature of the PEG-1000 rich phase at which it undergoes a transition from crystalline to isotropic ( $\mathrm{C}$ to $\mathrm{B}$ and $\mathrm{C}$ to $\mathrm{A}$ ) remains almost constant over the whole concentration range and only a small change in melting point is observed (in lower concentration of PEG-1000) in comparison to the pure PEG1000. This indicates that the crystalline phase is close to pure with only a minor E7 content.

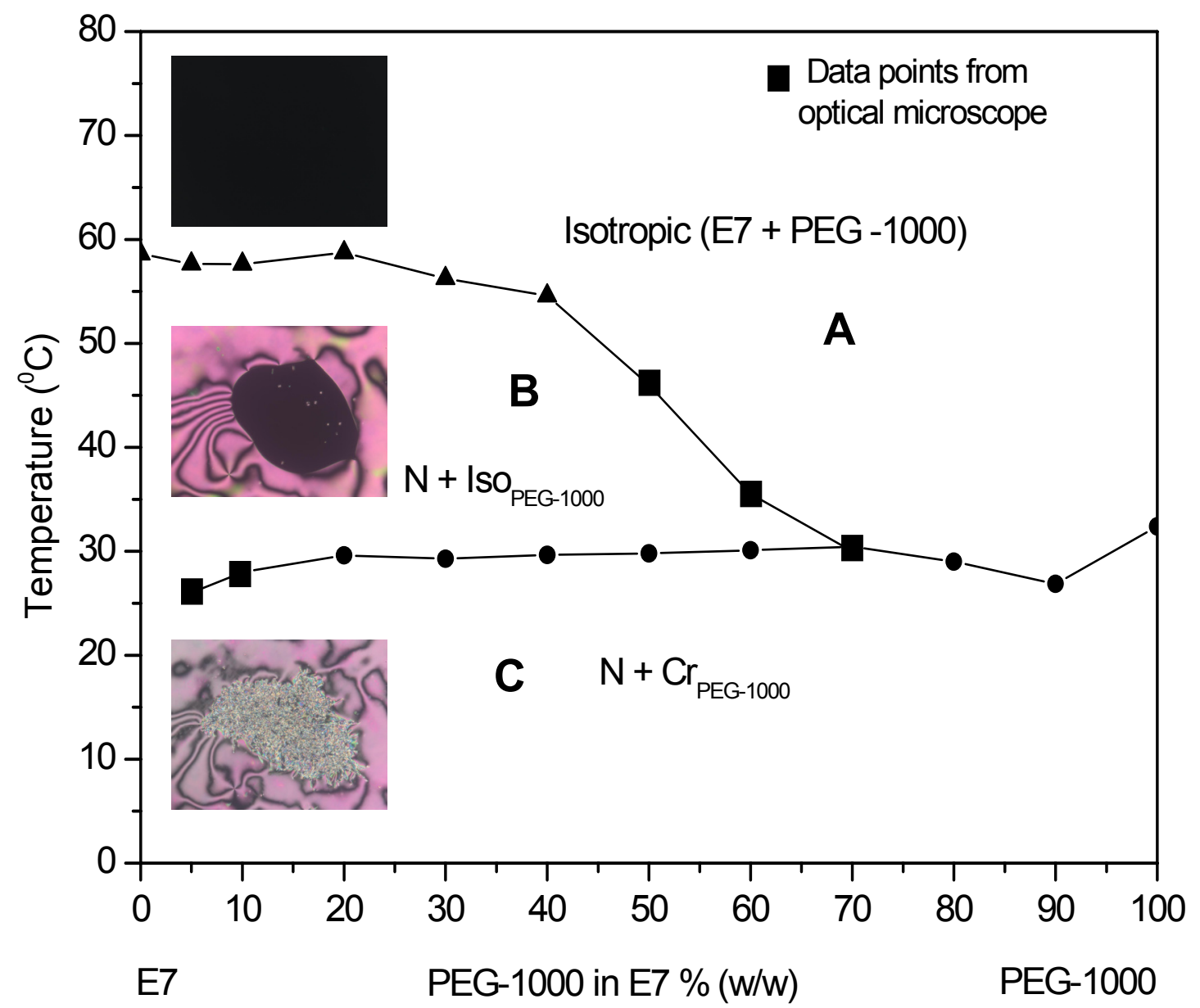

Figure 6.10: Binary phase diagram of PEG-1000 and E7, 'N' denotes nematic phase, 'Iso' the isotropic phase and ' $\mathrm{Cr}$ ' the crystalline phase. The optical micrographs shows the corresponding phases viewed between crossed polarizers with a rubbed cell at $45^{\circ}$ having $80 \%$ E7 and $20 \%$ PEG-1000. 
At this point it is worthwhile to note that the crystalline melting temperature of the E7 mixture is at around $-10{ }^{\circ} \mathrm{C}$. It also should be considered that E7 in itself is a mixture of four components. The solubility and interaction of these components with PEG-1000 might be mutually somewhat different, although no major differences are expected based on their similarity in chemical structure.

From the phase diagram it can be conclude that the selected blend of E7 and PEG-1000 is suited for further studies with surface controlled phase separation. Not only the transition temperature conveniently located, also the fact that a close to pure E7 phase is formed is of importance for its electro-optical switching characteristics. A minor drawback might be the crystalline melting temperature of the PEG-1000 which is just above room temperature. This is not an issue for our model studies, but might be detrimental for later applications.

\subsubsection{Phase behavior of LC mixtures (PEG-1000/E7) on bi-polymer surfaces}

\subsubsection{PTFE- Homeotropic polyimide}

In the previous sections the patterned alignment of LC on bi-polymer surface were investigated. This section describes the demixing of PEG-1000/E7 on the patterned PTFE/homeotropic polyimide surface. Cells with homeotropic polyimide and PTFE patterns were filled with PEG-1000/E7 mixtures to study the migration of these components in these bi-polymer surfaces. The two substrates had orthogonal PTFE deposition directions. The composition of PEG-1000/E7 (20\% PEG-1000 in E7), was selected from the phase diagram. In the cell the area of PTFE was much larger than that of the polyimide, in correspondence to the expected phase separated volumes and their anticipated spatial positioning. Figure 6.11 shows the optical microscopy image of the cell between crossed polarizers. In the figure three different areas are visible, PTFE/PTFE, PTFE/PI and PI/PI. Small part of the E7 is visible in the PTFE layer as white spots (TN area). Part of the LC is also present on the polyimide layer on both sides giving a homeotropic orientation. It is attempted to obtain migration of material to 
different surfaces dependent on a match and or mismatch in surface tension polarity. As can be concluded from Figure 6.11 that only partial demixing was observed. The areas enclosed by two polyimide areas contain mainly PEG-1000, but some contamination with phase separated E7 droplets could not be avoided as can be seen in the PI-PI area of figure 6.11. In the areas with PTFE surfaces, phase separated mixtures of liquid crystal and PEG-1000 are observed where the PEG-1000 are expelled from the E7 droplets and form coagulated edges around the domain boundaries of the liquid crystal. So there is a trend towards the desired structure, but phase separation and materials transport are not sufficient yet to be useful. It is anticipated that due to low polarity difference between the polyimide and the PTFE the migration of materials are not driven to completion.

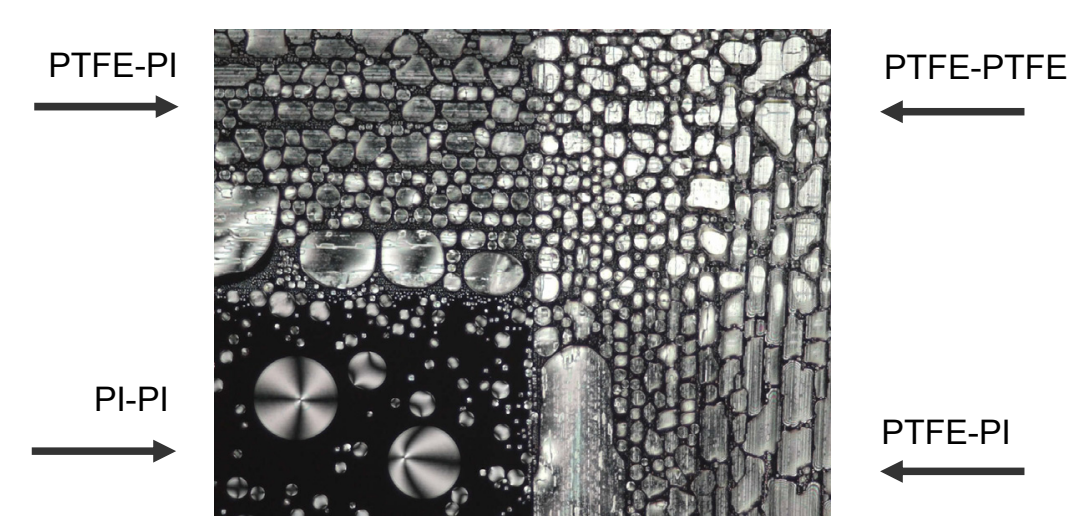

Figure 6.11: De-mixing of PEG-1000 and E7 on PTFE/Homeotropic polyimide surface viewed between crossed polarizers.

The homeotropic polyimides are known the have apolar aliphatic moieties near their surface effecting the perpendicular orientation of the liquid crystal, but most likely also limiting the driving force for material transfer. Planar aligning polyimides are known to have a better wettability for polar liquids and a better polarity contrast and corresponding better phase separation expected for these materials which will discussed in the next paragraph. 


\subsubsection{PTFE- Planar Polyimide}

The composition with lowest content of PEG-1000 was selected to fill an LC cell to study the phase separation behavior of these mixtures. The two substrates had orthogonal PTFE deposition directions. The PEG-1000 (20\%)/E7 mixture was filled in the PTFE/Planar polyimide cell by the capillary action at elevated temperature and was cooled slowly. Figure 6.12 shows the optical microscopy image of the cell viewed between the crossed polarizers.

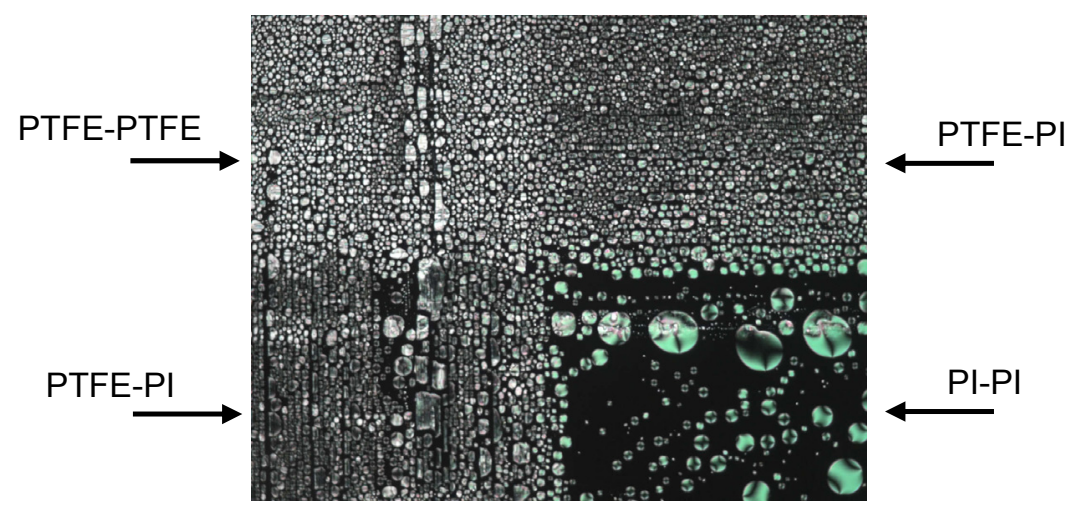

Figure 6.12: De-mixing of PEG-1000 and E7 on a patterned PTFE/Planar polyimide surface. Micrograph recorded between two crossed polarizers.

From the figure it is clear that the migration is not complete in this case also. The PEG-1000 migrated to the polyimide surface and on the PTFE layers both E7 and PEG1000 are present. This shows the need of surfaces with an even larger difference in contact angle.

\subsubsection{PTFE- PEG-SH surfaces}

In the previous paragraphs it was anticipated that the structured phase separation can be promoted by a number of methods:

1. Selecting surfaces with enhanced difference in surface tension and/or polarity

2. Selecting liquid crystals polymer mixtures with an enhanced difference in polarity

3. Creating surfaces with smaller polar/apolar patterns promoting materials transport. 
In this paragraph a surface tension patterns is described that are created with alternative hydrophobic, hydrophilic surfaces and the phase separation behavior of LC mixtures on these surfaces. For this purpose PTFE was selected as the hydrophobic surface and polyethylene glycol with thiol (PEG-SH) end group as hydrophilic surface. The ITO coated glass substrate was sputter coated with gold, which can react with the thiol end group of polyethylene glycol. The gold coated surface was patterned with PTFE using the friction deposition process as mentioned before. In this study $0.5 \mathrm{~mm}$ wide patterns of PTFE were deposited on the substrate. This substrate was treated with the 2\% PEG-SH solution in ethanol. The regions between the PTFE layers react with the thiol molecules to create a local hydrophilic surface. Two such substrates are placed orthogonal to each other with respect to the PTFE deposition direction and a cell with a spacing of $18 \mu \mathrm{m}$ was constructed. Figure 6.13 shows a schematic diagram of the cross section of glass substrate with the different layers. The cell was filled with a mixture of liquid crystal and polyethylene glycol and the migration of the mixtures was investigated.

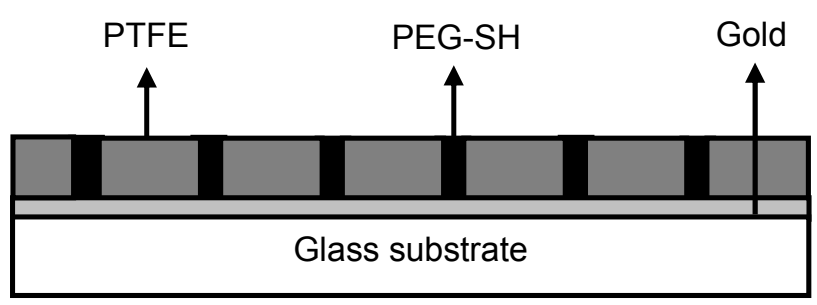

Figure 6.13: Schematic representation of gold/glass substrate with alternating regions of PTFE and PEG-SH.

A mixture containing $20 \%(\mathrm{w} / \mathrm{w})$ of PEG-1000 in E7 was selected to study the migration of these components on pre-patterned surfaces with differences in surface polarity. Due to the presence of the PEG-SH in the surface pattern, the PEG-1000 has an increased affinity to migrate to that surface and E7 is expected to migrate to the PTFE surface. In the optical micrographs in Fig. 6.14, it is shown that a good migration of the components is obtained. A twisted nematic configuration is observed where there is PTFE on both sides; outside this area a predominant presence of PEG-1000 is found. 


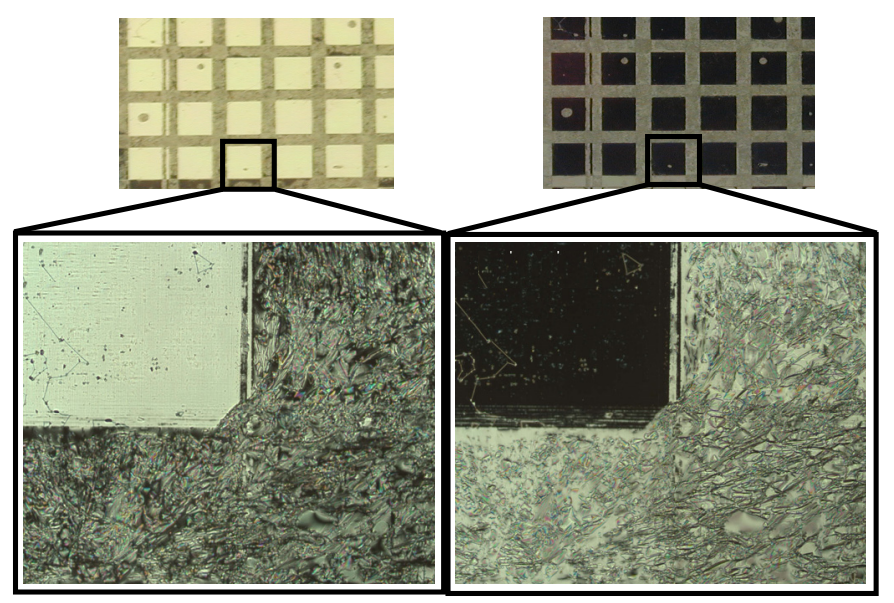

(a)

(b)

$20 \%(w / w)$ PEG1000 in E7

Figure 6.14: Phase separation of PEG-1000 in E7 on PTFE/PEG-SH surface, viewed between (a) crossed polarizers and (b) parallel polarizers.

Figure 6.15 shows the optical micrograph of the PTFE/PTFE TN area under an applied electric field $(5 \mathrm{~V})$. There are some small domains visible at $5 \mathrm{~V}$ probably due to the presence of very small amount of PEG-1000 trapped in this E7 phase. Outside the PTFE/PTFE area small amount of E7 is also present which is affected by the electric field as obvious from the change in contrast in those areas.

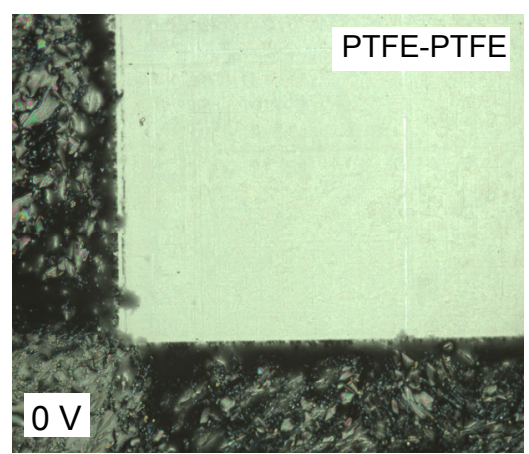

(a)

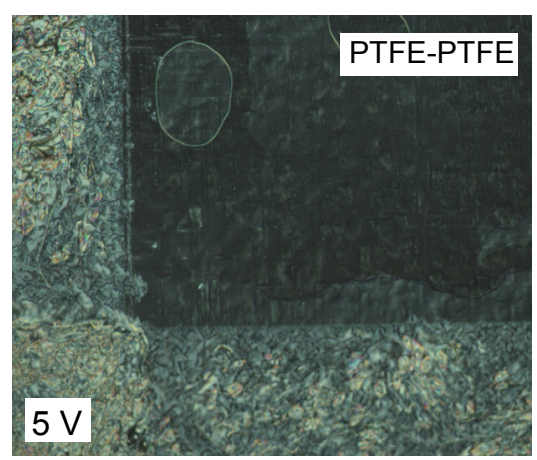

(b)

Figure 6.15: The migration of PEG-1000/E7 mixture on PTFE/PEG-SH surface viewed between cross polarizers in the off-state (a) and on-state (b). 
The electro-optical characteristics of the TN area in the PTFE/PTFE areas are shown in Fig.6.16. The threshold voltage is found to be $1.7 \mathrm{~V}$, the transmission curve more slowly approaches a zero value if compared to the transmission curve in Fig.6.9 (pure E7 on a PTFE substrate). This is probably related to the presence of a small amount of PEG-1000 in the E7 phase in the TN area.

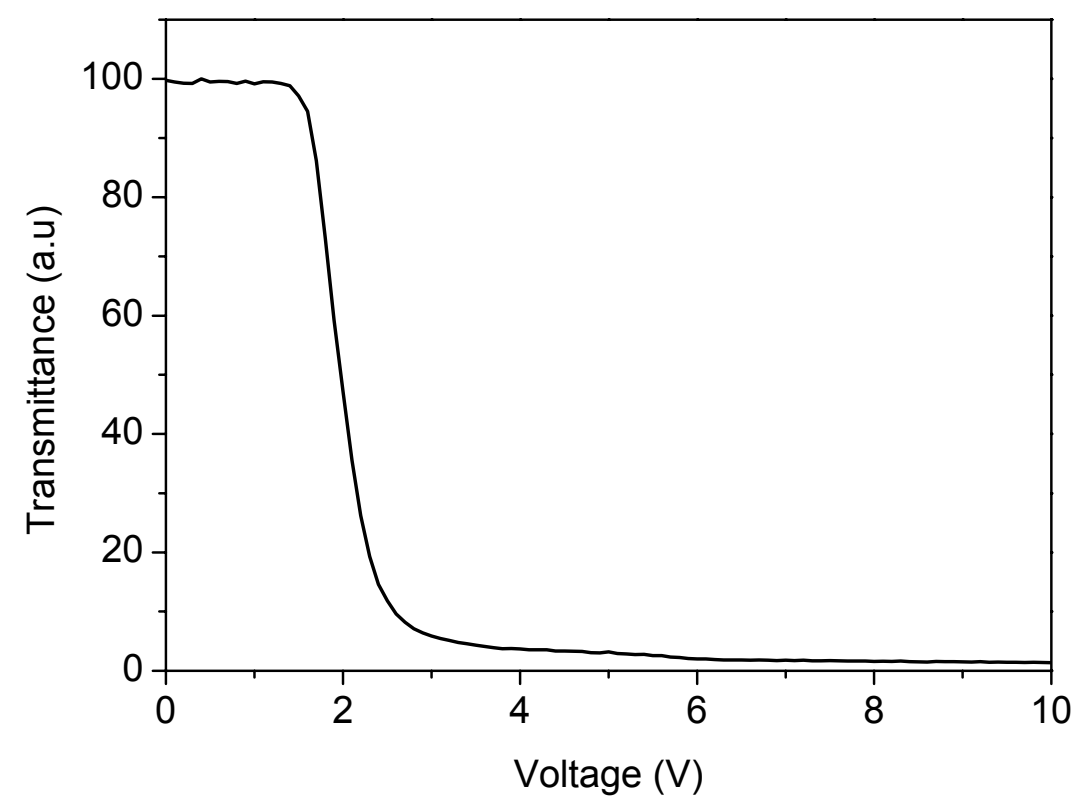

Figure 6.16: Transmission characteristics of TN cell from PTFE with E7, obtained by the migration of 67 from the E7/PEG-1000 blend.

The above described experiments illustrate that the spatially controlled phase separation of mixtures of a liquid crystalline material and a very polar polymer is experimentally feasible. Especially the use of patterned and friction deposited PTFE is beneficial in obtaining well defined structures. However, the use of friction deposited PTFE also imposes rather severe constraints i.e. PTFE is a rather intractable polymer which is insoluble in all common solvent. A rather crude method was therefore used to generate patterns via mechanical structuring of the PTFE rods. For instance, this results in patterns with a large pitch which cannot be reduced due to processing problems and, more importantly, due to the rather poor mechanical properties of the PTFE. As a 
consequence, gratings with a period in the micron range cannot be produced. New structuring techniques should be explored to generate the derived patterns in the proper geometry and dimensions. The use of small periods in the PTFE also can enhance the demixing of LC mixtures.

\subsection{Conclusions}

The spatially controlled phase separation of polymer/liquid crystal mixtures was investigated in the presence of surfaces with pre-tuned patterns of hydrophilic and hydrophobic regions. As a model mixture a blend of a very polar polymer and an amphiphilic liquid crystal was used. The phase behavior of this blend was investigated and it was found that the transition temperature of PEG-1000 from isotropic to crystalline phase is almost constant for a wide range of compositions. A rather poorly defined morphology was obtained in the case of homeotropic polyimide/PTFE and planar polyimide/PTFE. However, in the case of PEG-SH/PTFE combination a well defined pattern was observed and it was found that the hydrophilic polymer had a strong tendency to migrate to the PEG-SH areas while the liquid crystal rich phase migrated to the friction deposited PTFE layers. The liquid crystal preserved its ability to align and consequently twisted nematic patterns of liquid crystal in an isotropic PEG-SH matrix were produced. The TN area formed by the phase separated liquid crystal on PTFE/PTFE can be switched by the application of an electric field which opens the possibility to produce patterned and switchable optical components such as gratings.

\subsection{References}

[1] M. Boltau, S. Walheim, J. Mlynek, G. Krausch, U. Steiner, Nature 391, 877 (1998).

[2] S. Walheim, M. Boltau, J. Mlynek, G. Krausch, U, Steiner, Macromolecules 30, 4995 (1997).

[3] E. Schaffer, T. Thurn-Albrecht, T. P. Russell, U. Steiner, Nature 403, 874, (2000).

[4] A. Karim, J. F. Douglas, B. P. Lee, S. C. Glotzer, J. A. Rogers, R. J. Jackman, E. J. Amis, G. M. Whitesides Phys. Rev. E 57, R6273 (1998). 
[5] A. d'Alessandro, R. Asquini, C. Gizzi, R. Caputo, C. Umeton, A. Veltri, A. V. Sukhov, Optic. Lett. 29 (12), 1405 (2004).

[6] Y. Boiko, J. Eakin, J. Vedrine, G. P. Crawford, Optic. Lett. 27(19),1717 (2002).

[7] G. Lester, H. Coles, Mol. Cryst. Liq. Cryst. 312, 13 (1998).

[8] J. C. Wittmann, P. Smith, Nature 352414 (1991).

[9] J. R. Dennis and V. Vogel, J. Appl. Phys. 83, 5195 (1998).

[10] J. C. Wittmann, S. Meyer, P. Damman, M. Dosiere, H-W. Schmidt, Polymer 36, 3545 (1998).

[11] H. Hansama, F. Motamedi, P. Smith, P. Hansma, J. C. Wittmann, Polymer 33, 647 (1992).

[12] R. Hattori, Y. Aoki, T. Sugano, J. Shirafuji, T. Fujiki, Jpn. J. Appl. Phys. 36, 819 (1997).

[13] H. Suzuki, K. Oiwa, A. Yamada, H. Sakakibara, H. Nakayama and S. Mashiko, Jpn. J. Appl. Phys. Part 2, 34, 3937 (1995). 


\section{Technology Assessment}

The patterning of surfaces for the alignment of liquid crystals has important applications in liquid crystal displays, telecommunication devices and security elements. The most ubiquitous applications of patterned alignment are found in liquid crystal displays, where patterning is performed on the sub-pixel level in order to average out the strong optical anisotropy which is introduced by the liquid crystal orientation. This results in liquid crystal displays with a wide viewing angle and this is often achieved without using compensator films. There are several approaches in which one can prepare patterned surfaces for liquid crystal alignment, including photolithographic techniques, photoalignment using lithographic masks or holographic methods, selected deposition of $\mathrm{SiO}_{2}$ and nano-scratching of polyimide. ${ }^{1,2}$ Unfortunately, these processes are time consuming and expensive and exotic materials are often required.

In this thesis a new method is described for the patterned alignment of liquid crystals, which is coined as microrubbing ( $\mu$-rubbing) method. The $\mu$-rubbing process uses a metallic sphere to physically rub a polymeric alignment layer to create patterns. This method is very effective in creating patterns on the micrometer scale, which is the most relevant length scale for sub-pixelation. In this study three different polyimides are employed, one planar polyimide and two different types of homeotropic polyimide.

In an initial study, the $\mu$-rubbing was performed on planar polyimide to create well defined patterns with low surface pretilt. In fact, the pretilt was so low $\left(<1^{\circ}\right)$ that it was impossible to create a multidomain structure. Of course, this is a major limitation in the production of multidomain, twisted nematic displays with a wide viewing angle, a considerable effort was devoted to finding other routes for manufacturing multidomain displays (see later). Nevertheless, it was also shown that the alignment of liquid crystals on planar polyimides which were pre-rubbed with a velvet cloth can be erased by the $\mu$ rubbing process and well-defined patterns were obtained. These alignment patterns are potentially useful in the manufacturing of security features with combination of security leves or for personalized security cards. 
In the second part of the study the $\mu$-rubbing technique was used for the fabrication of multidomain twisted nematic display from a homeotropic polyimide. The $\mu$-rubbing of this homeotropic polyimide resulted in a pretilt angle of $9.8^{\circ}$ from the surface. The multidomain structure fabricated from this homeotropic polyimide was found to be unstable at $0 \mathrm{~V}$, but it is stable above $1.2 \mathrm{~V}$, which indicates the importance of a voltage initialization process. The multidomain structure gives a wide viewing angle and the electro-optical characteristics are independent of change in wavelength, which is suitable for full color display applications.

Currently used liquid crystal displays for desktop computer monitor and television screens have a wide viewing angle and are based on vertically aligned or in-plane switching mode. Here the $\mu$-rubbing technique was also used to create a multidomain twisted vertically aligned display with wide viewing angle and low color dispersion. A common problem of all vertically aligned displays, independent of mode, is that there is significant light leakage in the zero voltage state at large angles due to the optical retardation of vertically aligned liquid crystals at oblique angles. ${ }^{3}$ This multidomain twisted vertically aligned display also exhibits this light leakage at large angles. However, because of its symmetry, a simple negative $c$-plate compensation, providing an optical anisotropy complementary to that of the vertically aligned liquid crystals, which behaves as a positive $c$-plate, can be used to minimize the light leakage and improve the viewing volume in the zero voltage state. ${ }^{4}$

Another interesting feature of $\mu$-rubbing technique is the tailoring of pretilt angle and sub-pixel size by controlling the load and the diameter of the metallic sphere. The use of further small sphere with suitable pressure, it is possible to make displays with very small pixel size to increase the resolution of displays.

In this study metallic sphere of $1 \mathrm{~mm}$ was used to get a sub-pixel dimension of $45 \mu \mathrm{m}$. Here a single metallic sphere was used, but in practical display applications multi sphere system can be used (comb-like configuration) to pattern large area display panels. Computer controlled X, Y coordinate moving systems can be used to automate the $\mu$ rubbing process. In this kind of setup the pressure applied and the speed of writing etc. 
can be precisely controlled. Further studies are needed to evaluate the stability and performance of the thin film transistors (TFT's) under the polyimide layer. The possible development electro-static charge during the $\mu$-rubbing process is also taken into account. Suitable design of the $\mu$-rubbing setup and implementation of special anti-static method can solve this problem. This technique can be scaled up and used in the production of large area displays. The implementation of this technique in an LCD assembly line reduces the production cost of the LCDs.

The last part of this thesis describes the pattern formation of liquid crystal mixtures on prepatterned surfaces with hydrophilic and hydrophobic regions. A migration of the components of a de-mixing mixture of a liquid crystal and a polymer to surfaces with similar polarity was observed. A pattern is obtained which is active in the sense that it can be switched from bright to dark state by the application of an electric field. These kinds of micro-patterns are potentially usefull as switchable gratings in telecommunication field. Additional studies are, however required to reduce the pattern size to micron level.

Summarizing, the $\mu$-rubbing techniques described in this thesis open new routes for the manufacturing of multidomain liquid crystal display with improved viewing angle. The $\mu$-rubbing technique is straightforward, flexible, economical and simple to execute. The uniqueness of this technique is that it can be used for the fabrication of different kinds of display modes, like twisted nematic and vertically aligned displays. Moreover, the same technique can probably also be applied to multidomain super twisted nematic displays and multidomain hybrid aligned systems.

\section{References}

[1] J. Chen, P. J. Bos, D. L. Johnson, D. R. Bryant, J. Li, S. H. Jamal, J. R. Kelly, J. Appl. Phys. 80, 1985 (1996).

[2] B. Wen, M. P. Mahajan, C. Rosenblatt, Appl. Phys. Lett. 76, 1240 (2000).

[3] H. Yoshida, T. Seino, Y. Koike, Jpn. J. Appl. Phys. 36, L1449 (1997).

[4] M. Lu, K. H. Yang, Jpn. J. Appl. Phys. 39, L412 (2000). 


\section{Summary}

Liquid crystal displays (LCDs) are increasingly used as a man-machine interface in applications such as telephones, personal organizers, laptops, desktops computers and television screens. The enormous success of LCDs is predominantly related to its flatness, low power consumption and light weight. Nowadays, a variety of different switching principles are used based on twisted nematic (TN), super twisted nematic (STN), vertically aligned (VA) and electrically controlled birefringence (ECB) and several addressing schemes are employed (passive and active matrix) to meet the ever increasing requirements with respect to switching voltage, switching kinetics, resolution and viewing angle. Traditionally, LCDs exhibit disadvantages with respect to viewing angle and color inversion. In modern LCDs these problems are circumvented by external compensation films which correct for the intrinsic optical anisotropy within the electrooptical cell. Unfortunately, the production of these films is laborious and expensive and a need persists for new methods which are compatible with cheap and large scale production processes.

In LCDs, alignment layers based on polyimides are extensively used to properly align the liquid crystals. Here, a new method is presented for the micro-patterning of these alignment layers. The main objective is to create multidomain pixels with a different director profile of the LCs in each sub-pixel. As a consequence, it is expected that wide viewing angle LCDs are obtained without using external compensation films.

This thesis demonstrates the use of microrubbing ( $\mu$-rubbing) as a new tool for the micro-patterning of polyimides. The process uses a metallic sphere to mechanically rub a polyimide layer under well defined conditions to create alignment patterns with the appropriate dimensions (length/width) for sub-pixelation (20-50 $\mu \mathrm{m})$. It was found that $\mu$ rubbing of planar polyimides induces a planar alignment in liquid crystals with a typical pretilt between $0-0.7^{\circ}$. The $\mu$-rubbing erases the pre-rubbing history of the planar polyimide. Electro-optical cells were constructed and different liquid crystal configurations were observed such as planar and twisted nematic. The 2dimMOS computer program was used to study the orientation of directors in different areas and the 
theoretical results were compared with the experimental observations. The electro-optical transmissions of liquid crystal cell were also simulated using extended Jones matrix method. The experimental and simulated results are in good agreement. It was also attempted to produce a four-domain twisted nematic display with improved viewing angle. The planar polyimide was used to construct a four-domain TN display, but due to very low pretilt angle it was impossible to observe a four-domain structure. Therefore different routes were explored to manufacture stable multidomain pixels using different types of polyimides.

A second study was performed on the $\mu$-rubbing of homeotropic polyimides. The prime objective here was to generate alignment layers with a high pretilt for the manufacture of stable multidomain pixels. The $\mu$-rubbing of homeotropic polyimide, AL-75114 resulted in planar alignment with a surface pretilt of $9.8^{\circ}$ at a load of $150 \mathrm{~g}$. The variation of pretilt angle and the electro-optical response with respect to the applied load were investigated and it was found that a tailoring of pretilt angle is possible in a small range of pretilt angles (from $9.8^{\circ}$ to $16^{\circ}$ ). A multidomain pixel was fabricated using this homeotropic polyimide and its electro-optical properties were studied. In the case of this TN display it was found that a voltage initialization process stabilizes the four-domain structure. For this initialization process the voltage was increased slowly from $0 \mathrm{~V}$ to $5 \mathrm{~V}$ to reach on-state (dark state). Subsequently, the voltage was reduced gradually and a stable four-domain structure was observed at a voltage above $1.2 \mathrm{~V}$. Below $1.2 \mathrm{~V}$, the four-domain essentially transforms into a two-domain reverse tilt sample. The pretilt angle at $1.2 \mathrm{~V}$ was $\sim 30^{\circ}$ which completely stabilizes the motion of disclination lines to form stable four-domain structure. In the region from 1.2-5 volt, the four-domain structure gives symmetric isointensity curves, which corresponds to a drastic improvement in viewing angle. The dependency of white color chromaticity on azimuthal viewing directions is also reduced substantially due to the self-compensation effects in the four-domain cell. The viewing angle of multidomain display was simulated and compared with the experimental results using extended Jones matrix method. The formation of disclination lines between the boundaries of domain in a pixel was also 
observed in the calculations of the director orientations using the 2dimMOS program and it was in good agreement with the experimental observation. A thermal aging study was performed to evaluate the stability of the alignment of liquid crystal and it was found that the alignment layer is robust and can be used in high-end display applications. The above described results illustrate that a very high pretilt $\left(>30^{\circ}\right)$ is required to obtain multidomain TN pixels which are also stable at low voltages and zero voltage.

An additional study was therefore performed to get a stable four-domain structure using a different homeotropic polyimide (Nissan Sunever SE 1211). The $\mu$-rubbing of this polyimide resulted in a pretilt angle of $89^{\circ}$. In accordance with expectations it was found that stable, four-domain and vertically aligned pixels were generated. In this case liquid crystal with negative dielectric anisotropy was used. The four-domain twisted vertically aligned display produced exhibits good contrast and excellent viewing angle characteristics. Moreover this four-domain display exhibits faster switching time compared to the twisted nematic display. The experimental observations were also verified using optical simulation methods for the viewing angle and for the electro-optical characteristics and they are in good agreement.

Finally, a study was performed to produce alignment patterns which simultaneously also posses a surface tension pattern. It was attempted to generate controlled migration of blends of materials which de-mix on these alignment patterns to obtain, for instance, switchable and/or polarization-selective gratings for telecommunication purposes. It was observed that good migration is achieved with liquid crystal E7 and polyethylene glycol (E7/PEG-1000) mixtures on polytetrafluoroethylene and mercaptopolyethylene glycol (PTFE/PEG-SH) surface patterns.

In conclusion, it was shown that the $\mu$-rubbing of alignment layers for liquid crystals is a versatile technique for the fabrication of multidomain structures with improved display properties with respect to wide viewing angle, contrast ratio and low color dispersion. The $\mu$-rubbing technique is simple to perform, economical and compatible with the currently used manufacturing processes. 


\section{Samenvatting}

Vloeibaar kristallijne beeldschermen (LCDs) worden steeds meer gebruikt in toepassingen zoals telefoons, digitale agenda's, laptops, computers en televisies. Het enorme succes van LCDs is voornamelijk te danken aan de platte vorm, het laag stroom verbruik en het lichte gewicht. Tegenwoordig worden er verschillende schakel principes gebruikt zoals getwist nematisch (TN), super getwist nematisch (STN), verticale oriëntatie (VA) en elektrisch gecontroleerde dubbelbreking (ECB). Ook worden verschillende manieren van aansturing (passief en actief) gebruikt om te voldoen aan de toenemende eisen op het gebied van schakel voltage, schakel snelheid, resolutie en kijkhoek.

Conventionele LCDs bezitten een beperkte kijkhoek en zij vertonen kleuren inversie. In moderne LCDs zijn deze problemen opgelost door gebruik te maken van externe compensatie films die corrigeren voor de intrinsieke optische anisotropie van de elektro-optische cel. De productie van deze films is echter zeer bewerkelijk en duur en er is behoefte aan nieuwe, goedkope en effectieve methoden voor het vervaardigen van LCDs met een brede kijkhoek.

In de meeste LCDs wordt gebruik gemaakt van gewreven oriëntatie lagen gebaseerd op polyimiden om vloeibare kristallen te oriënteren. In dit proefschrift wordt een nieuwe methode beschreven om deze oriëntatie lagen te patroneren. Het hoofddoel is om iedere pixel van een LCD te verdelen in meerdere domeinen met een verschillende oriëntatie. Hiervan wordt verwacht dat LCDs met een brede kijkhoek worden verkregen zonder gebruik te maken van een externe compensatie film.

In dit proefschrift is aangetoond dat micro-wrijven gebruikt kan worden om oriëntatie patronen aan te brengen in polyimides op micron niveau. De methode maakt gebruik van een metalen object voor het wrijven van een polyimide laag onder goed gedefinieerde condities. Oriëntatie patronen met de juiste dimensies (lengte/breedte) voor het vervaardigen van verschillende domeinen in pixels zijn verkregen. Aangetoond is dat micro-wrijven van planair polyimide een planaire oriëntatie geeft van vloeibare kristallen met een hoek (pretilt) van $0-0.7^{\circ}$ met het vlak van de film. Het micro-wrijven 
wist eerdere wrijfstappen met een zijden doek uit. Elektro-optische cellen zijn vervaardigd met diverse vloeibaar kristallijne configuraties in één cel zoals planair en getwist nematisch. Computersimulaties zijn gebruikt om de oriëntatierichting van vloeibare kristallen in verschillende domeinen gebieden te bestuderen en de theoretische resultaten zijn vergeleken met experimentele resultaten. De transmissie van de vloeibare kristallijne cellen als functie van schakelvoltage is gesimuleerd met de Jones matrix methode. De experimentele en gesimuleerde resultaten zijn in goede overeenstemming. Ook is getracht om een getwist nematisch beeldscherm te maken met een brede kijkhoek. Echter, door de lage initiële hoek van het vloeibare kristal met het vlak van de film is het niet mogelijk is om stabiele pixels te verkrijgen die onderverdeeld zijn in verschillende domeinen. Daarom zijn er andere methoden onderzocht om stabiele domeinvorming te verkrijgen door gebruik te maken van andere polyimiden.

In een tweede studie zijn homeotrope polyimides gebruikt voor het vervaardigen van micro-gewreven oriëntatielagen die een hoge hoek met het vlak van de film induceren in vloeibare kristallen. De voornaamste doelstelling hiervan was het vervaardigen van pixels met stabiele en goed gedefinieerde domeinen. Het micro-wrijven van de homeotrope polyimide AL-75114 resulteerde in een planaire uitlijning met een pretilt van 9.8 graden bij een belasting van 150 gram. Bovendien is gebleken dat de pretilt ingesteld kan worden d.m.v. procescondities zoals wrijfdruk in een gebied tussen 9.8 en 16 graden. Twisted nematische cellen zijn vervaardigd en er is gevonden dat pixels met een stabiele domein structuur worden verkregen bij spanningen tussen 1.2 en 5 Volt. Een voltage van 1.2 volt correspondeert met een hoek van het vloeibaar kristal met het vlak van het substraat van ongeveer 30 graden hetgeen voldoende is om disclinaties tussen domeinen te stabiliseren. De electro-optische cellen hebben symmetrische isointensiteit krommen hetgeen overeenkomt met een sterke verbetering in kijkhoek. De kijkhoek van deze electro-optische cellen is vergeleken met computersimulaties en een zeer goede overeenkomst met experimentele resultaten is verkregen. Bovendien werd ook het optreden van stabiele disclinaties correct voorspeld. Een oriënterende studie is 
ook uitgevoerd m.b.t. de stabiliteit van de micro-gewreven oriëntatielagen en het is aangetoond dat deze lagen voldoende stabiel zijn voor toepassingen in b.v. televisie.

Een tweede homeotrope polyimide (Nissan Sunever SE 1211) is bestudeerd om een LCD te verkrijgen met een stabiele domeinstructuur bij alle spanningen (0-5 Volt). Het micro-wrijven van deze polyimide resulteerde in een hoek met de wrijfrichting van 89 graden en stabiele domeinen worden gevormd in deze verticaal uitgelijnde LCDs. In dit geval zijn vloeibare kristallen met een negatieve dielectrische anisotropie gebruikt en electro-optische cellen zijn vervaardigd met een uitstekende en zeer brede kijkhoek en een hoog kontrast. Bovendien is gevonden dat deze cellen een zeer snelle schakelkinetiek bezitten.

Tenslotte is er een onderzoek uitgevoerd naar de migratie van fasescheidende systemen die een vloeibaar kristal bevatten in de aanwezigheid van een oppervlaktespannings patroon. Het is gebleken dat mengsels van een vloeibar kristal (E7) met polyethyleenglycol gebruikt kunnen worden voor het vervaardigen van patronen in ontmengende systemen. Deze schakelbare systemen kunnen mogelijkerwijs gebruikt worden als schakelbare gratings voor telecommunicatie toepassingen. 


\section{Publications}

1. S. Varghese, G. P. Crawford, C. W. M. Bastiaansen, D. K. G. de Boer, D. J. Broer, "Microrubbing technique to produce high pretilt multidomain liquid crystal alignment," Appl. Phys. Lett. 85, 230 (2004).

2. S. Varghese, S. Narayanankutty, C.W.M. Bastiaansen, G. P. Crawford, D.J. Broer, "Patterned Alignment of Liquid crystals by $\mu$-Rubbing," Adv. Mater. 18, 1600 (2004).

3. S. Varghese, G. P. Crawford, C. W. M. Bastiaansen, Dick K. G de Boer, D. J. Broer, "Multi-configurations in nematic liquid crystal films: A microrubbing approach," Mol. Cryst. Liq. Cryst. 429, 55 (2005).

4. S. Varghese,G. P. Crawford, C. W. M. Bastiaansen, Dick K. G de Boer, D. J. Broer, "High pretilt four-domain twisted nematic liquid crystal display by microrubbing: Process, characterization and optical simulation," J. Appl. Phys. 97, $53101(2005)$.

5. S. Varghese, G. P. Crawford, C. W. M. Bastiaansen, Dick K. G de Boer, D. J. Broer, "Optical investigation of disclination lines in multidomain twisted nematic liquid crystal display created by microrubbing," Mol. Cryst. Liq. Cryst. (in press), (2005).

6. S. Varghese, G. P. Crawford, C. W. M. Bastiaansen, Dick K. G de Boer, D. J. Broer, "Four-domain vertically aligned liquid crystal pixels using microrubbing," Appl. Phys. Lett. (in press), (2005).

7. S. Varghese, G. P. Crawford, C. W. M. Bastiaansen, Dick K. G de Boer, D. J. Broer, "Four-domain twisted vertically aligned liquid crystal pixels using microrubbing: Process, characterization and optical simulation," J. Appl. Phys. (to be submitted).

8. S. Varghese, S. Narayanankutty, C.W.M. Bastiaansen, G. P. Crawford, D. J. Broer, "Patterned alignment of liquid crystals," SPIE proceedings, San Jose, USA, 5289, 206 (2004).

9. S. Varghese, G. P. Crawford, C. W. M. Bastiaansen, Dick K. G de Boer, D. J. Broer, "Multidomain twisted vertically aligned display by microrubbing and its simulations," SID’05 digest of technical papers, Boston, USA (2005).

10. S. Varghese, S. Narayanankutty, C. W. M. Bastiaansen, D. J. Broer, "Friction deposition of PTFE on rubbed polyimides," Proceedings, Advances in Polymer Technology-02, Cochin, India, 110 (2002). 


\section{Acknowledgements}

First of all I would like to thank His All Mighty for giving me an opportunity to do my research in a nice group here in the Netherlands.

I would like to express my sincere thanks to all the people who directly or indirectly helped me with my research. Firstly I would like to thank my promoter prof. dr. Dirk J. Broer for his support and guidance in carrying out this research. I am extremely indebted to my co-promoter, dr. Cees W. M. Bastiaansen, for his support throughout this project. I sincerely appreciate the motivation and support that he has given me during my research, especially during my stay in India and in my personal life. Cees, I will never forget it, bedankt. I would also like to extend my gratitude to my second promoter prof. dr. Piet Lemstra for his help and support during my time in the Netherlands.

Next I would like to express my deepest gratitude to prof. dr. Gregory P. Crawford from Brown University, in Providence, RI, USA for all the help he gave me

during his sabbatical in the Netherlands. Greg, you taught me the basic concepts of research and the art of writing articles. I really appreciate all of your help. Thank you very much.

I profoundly thank prof. dr. K.E George, the pioneer of this project and the former head of the Department of Polymer Science and Rubber Technology (PSRT) at Cochin University of Science and Technology (CUSAT) for giving me the opportunity to conduct my research. Sir, thank you for your support and prayers. I would also like to thank the head of PSRT, CUSAT, prof. dr. Rani Joseph for her support and help during my studies. I would like to thank my co-supervisor in PSRT, dr. Sunil K. Narayanankutty for his guidance and cooperation during my research. I also would like to extend my thanks to prof. dr. A. P Kuriakose (professor emeritus), dr. Eby Thomas Tachil, dr. Philip Kurian, dr. Thomas Kurian, dr. Honey John and Lity Alen Varghese, the faculty members of PSRT, for their best wishes. I would also like to express my gratitude to all the research scholars of PSRT, CUSAT for their help and words of encouragement. 
To Jan van Cranenbroek, the pioneer and the initiator of the PSRT project; it was his effort, patience and willingness that made this project a success. I would like to express my sincere thanks to him for this as well as for helping me to get settled in the Netherlands. I would also like to express my gratitude to the successor of the PSRT project Ir. Patrick van Schijndel. Patrick, thank you for all of the help you gave me during my studies. I would also like to thank dr. Uday Agarwal, the co-coordinator of the PSRT project.

My sincere thanks to dr. Dick K.G. de Boer from Philips Research Laboratories for the valuable discussions and help he gave me with the modeling aspects of my thesis. I also like to thank dr. Hugo Cornelissen, dr. Wouter Opets, Ir. Mark Hage and Rogier Cortie for their help during my visit to Philips Research Laboratories.

I would like to thank all the members of SKT group for their support and help especially the PICT group members - Carlos Sanchez (Universidad de Zaragoza, Spain) Chris Dunn (Merck, UK), Michael Escuti (NCU, USA), Ken Harris, Michael Debije, Matt Sousa (Brown University, USA), Carmen, Charlotte and Blanca. To my officemates, David, Pit, Chris Hendricks, Joost, Anastasia, Vidya, Lijing, Ko and Casper - I had a wonderful time sharing an office with you, thank you very much for the fond memories. My special thanks to David for helping me with AFM measurements and Chris van Heesch the '3D studio expert' for designing pictures. I would also like to thank Peter Minten of the GTD, TU/e for his help with the substrate patterning and Pauline Schmit, Anne Spoelstra for the microscopy techniques.

I am grateful to all my malayali friends Aneesh, Anoop, Babu, Dilna, Jojo, Merina, Prasanth, Subi, Tony, Vinu, Vipin, Vinod and my desi (Indian) friends Mano, M. K Singh, Nilesh, Sachin, for making Eindhoven feel more like home. I would also like to thank dr. Jacob Samuel, (VBL, Japan) and M. Melhado for their support and help.

Finally I dedicate this thesis to my Chachan and Ammachi for their moral and emotional support as well as their patience. I would also like to express my thanks to my sisters Sheeba and Seena as well as the rest of my family for their whole hearted support. 


\section{Curriculum Vitae}

Soney Varghese was born in Ezhupunna, Kerala, India on May $5^{\text {th }} 1974$. After finishing his Bachelors degree (B.Sc) in Chemistry from Mahatma Gandhi University in 1994, he joined the masters program (M.Sc) in Chemistry at the same university and completed his degree in 1996. In 1999 he finished his Master of Technology (M.Tech) in Polymer Technology from Cochin University of Science and Technology (CUSAT). He was working as a lecturer in the same university until 2001. In October 2001, he joined in the Polymers in Information and Communication Technology (PICT) group at the Chemistry and Chemical Engineering department of Eindhoven University of Technology (TU/e), The Netherlands for his Ph.D under the supervision of prof. dr. Dirk J. Broer and dr. Cees W. M Bastiaansen. The research leading to this thesis was supported by the collaboration between TU/e and Department of Polymer Science and Rubber Technology, CUSAT under the NUFFIC (Netherlands organization for international cooperation in higher education) sponsored "Joint financing program for cooperation in Higher Education (MHO)". 